\title{
Functional and diagnostic relevance of FGFR1-dependent signaling pathways in squamous cell lung cancer
}

\author{
Dissertation \\ for the award of the degree \\ “Doctor rerum naturalium" (Dr. rer. nat.) \\ of the Georg-August-Universität Göttingen \\ within the doctoral program (Molecular Medicine) \\ of the Georg-August University School of Science (GAUSS)
}

\author{
submitted by \\ Omar Elakad \\ from \\ Sharkia, Egypt
}

Göttingen 2020 


\section{Members of the thesis committee and examination board:}

\section{Prof. Dr. Henning Urlaub}

(First TAC member) Max Planck Institute for Biophysical Chemistry, Am Fassberg 11, 37077 Goettingen, Germany.

\section{Prof. Dr. Matthias Dobbelstein}

(Second TAC member) Institute of Molecular Oncology, University Medical Center Göttingen, Justus-von-Liebig-Weg 11, 37077 Goettingen, Germany.

\section{Prof. Dr. Fred S. Wouters}

(Third TAC member) Institute of Neuropathology, University Medical Center Göttingen, Waldweg 33, 37073 Goettingen, Germany.

\section{Further members of the examination board:}

\section{Prof. Dr. Argyris Papantonis}

Institute of Pathology, University Medical Center Göttingen, Robert-Koch-Strasse 40, 37075 Goettingen, Germany.

\section{Dr. Ufuk Günesdogan}

Department of Developmental Biology, University of Göttingen, Justus-von-Liebig Weg 11, 37077 Goettingen, Germany.

\section{Dr. Shiv Singh}

Department of Gastroenterology and Gastrointestinal Oncology, University Medical Center Göttingen, Robert-Koch-Strasse 40, 37075 Goettingen, Germany.

\section{Date of Disputation:}




\section{AFFIDAVIT}

Here I declare that my doctoral thesis entitled "Functional and diagnostic relevance of FGFR1-dependent signaling pathways in squamous cell lung cancer" has been written independently with no other sources and aids than quoted.

Omar Elakad

Göttingen,

27.05.2020. 


\section{List of publications}

\section{Published:}

a. Parts of this thesis have been published here:

Elakad, O., Lois, A. M., Schmitz, K., Yao, S., Hugo, S., Lukat, L., Bohnenberger, H. (2020). Fibroblast growth factor receptor 1 gene amplification and protein expression in human lung cancer. Cancer Med. doi:10.1002/cam4.2994.

b. Saha, S., Yao, S., Elakad, O., Lois, A.-M., Henric-Petri, H., Buentzel, J., Bohnenberger, H. (2020). UDP-glucose 6-dehydrogenase expression as a predictor of survival in patients with pulmonary adenocarcinoma. International Journal of Surgery Oncology, 5(2). doi:10.1097/ij9.0000000000000085

c. Buentzel, J., Yao, S., Elakad, O., Lois, A. M., Brunies, J., Konig, J., Bohnenberger, H. (2019). Expression and prognostic impact of alpha thalassemia/mental retardation $\mathrm{X}$-linked and death domain-associated protein in human lung cancer. Medicine (Baltimore), 98(31), e16712. doi:10.1097/MD.0000000000016712

\section{In preparation:}

d. Activation of Akt confers intrinsic and induced resistance to FGFR1 inhibition in lung cancer. (First author)

e. ANXA1, a favorable prognostic biomarker in squamous cell lung cancer. (First author)

f. The One Carbon Metabolic Landscape of Lung Cancer synthetic MTHFD2 as a therapeutic target for a subgroup of pulmonary adenocarcinoma patients. (Co-author) 
Dedicated to my father Prof. Dr. Mohamed Elakad who taught me that science is the basis of nations' renaissance

And

my mother Dr. Aziza el-khouly who was my biggest support system throughout this long journey 


\section{Acknowledgments}

At the end of this project, I would like to thank all the people who helped and supported me in making this thesis possible. I wish to thank Prof. Dr. Henning Urlaub (Max Planck Institute for biophysical chemistry, Goettingen) for accepting me as his PhD student and for his continuous supervision and help. My sincere appreciation and gratitude go to Dr. med Hanibal Bohnenberger (Institute of Pathology, UMG) for his continuous and constructive support and guidance throughout the thesis. The success of this thesis is attributable to Dr. Bohnenberger being a great scientist, supervisor and person.

I would like to thank Prof. Dr. Matthias Dobbelstein (Head of Institute of Molecular Oncology, University of Goettingen) for taking part in my thesis committee and for his constructive advices during my PhD. I am grateful to Prof. Dr. Fred S. Wouters (Institute for Neuropathology, University of Goettingen) for his supervision and help during my PhD. My thanks and appreciation go to Prof. Dr. Philipp Ströbel (director of Institute of Pathology, UMG) who accepted me as a student in his department and who supported my contract at the end of my PhD thesis. I appreciate the help of Prof. Dr. Thomas Oellerich and Uwe Plessman (Institute of Hematology and Oncology at University Hospital of Frankfurt) and their lab who helped me in sample preparation of LC-MS/MS analysis.

I would like to thank Sha Yao and Anne-Maria Lois (Institute of Pathology, UMG) for their help in diagnosing immunohistochemistry patient tissue samples. Many thanks for the nice work done at the lab of Prof. Dr. Hans-Ulrich Schildhaus (Institute of Pathology, UMG) by Sara Hugo who helped me analyzing fluorescence in situ hybridization samples of human tissues and cell lines. I am very grateful for the remarkable help and support I received from Dr. Stefan Küffer and Jennifer Appelhans (Institute of Pathology, UMG). I would like to thank Nada Ismael (AASTMT), Mahmoud AbouZeid (Dep. of Agricultural Economics, University of Goettingen) and Mohamed Ismael (Dep. of Molecular Biology, University of Goettingen) for their wonderful accompany and support throughout my PhD and I wish them all success in their studies. Finally, I thank the German Cancer Aid "Krebshilfe" for funding the current project and the National Institute of Health $(\mathrm{NIH}$, $\mathrm{NCl}$, Frederick, MD 2170) for supporting us with the DMS114 lung cancer cell line. 


\section{Table of contents}

List of publications iv

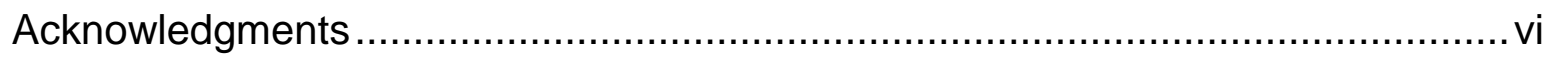

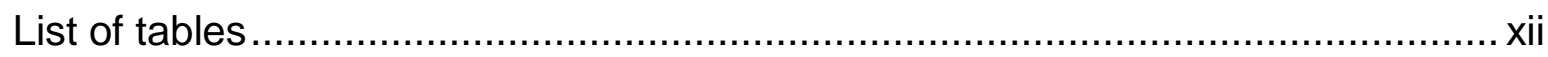

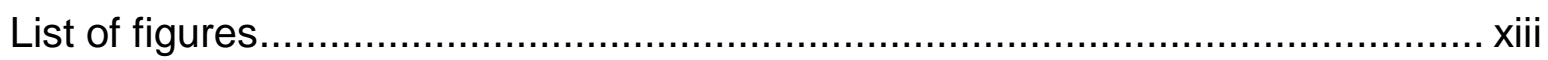

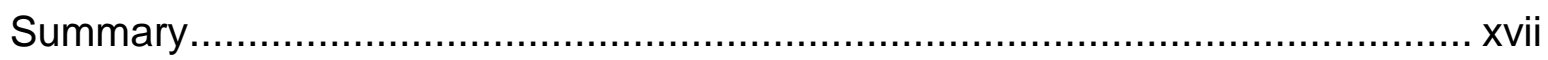

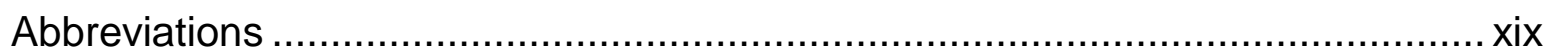

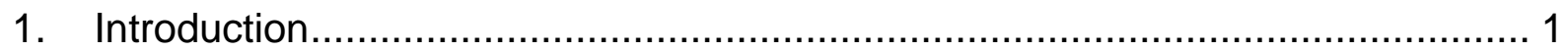

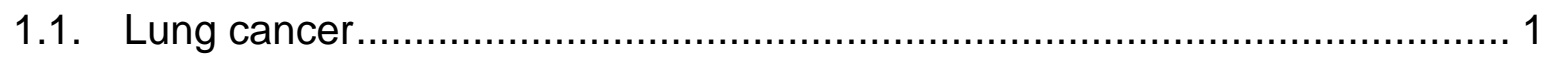

1.1.1. Prevalence and Mortality ........................................................... 1

1.1.2. Histological classification............................................................. 1

1.1.3. Squamous cell lung cancer (SQCLC) ……................................... 2

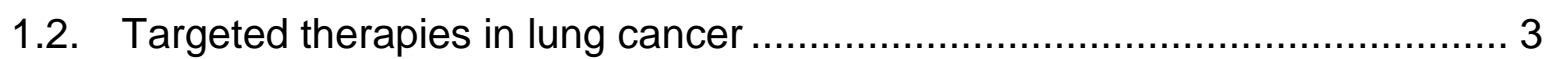

1.2.1. Significance of molecular targets ................................................... 3

1.2.2. Receptor tyrosine kinases (RTKs) ................................................ 4

1.2.3. Targeted therapy in non-small cell lung cancer (NSCLC) .................... 5

1.2.4. Fibroblast growth factor receptor 1 (FGFR1)................................... 6

1.3. Targeting FGFR1 in squamous cell lung cancer (SQCLC) ......................... 8

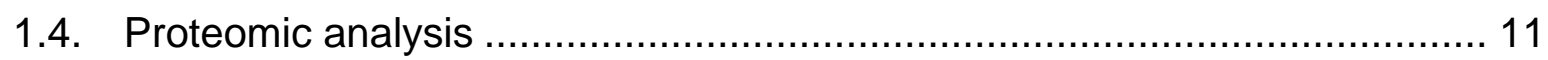

1.4.1. Proteomic analysis in cancer research......................................... 11

1.4.2. Liquid chromatography-coupled electrospray ionization mass spectrometry (LC-MS/MS) ............................................................... 13

1.5. Stable Isotope Labeling with Amino acids (SILAC) labeling ...................... 14

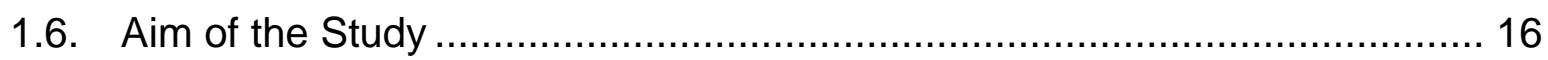

2. Materials and Methods ……................................................................. 17

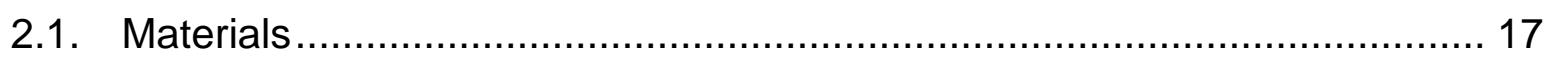

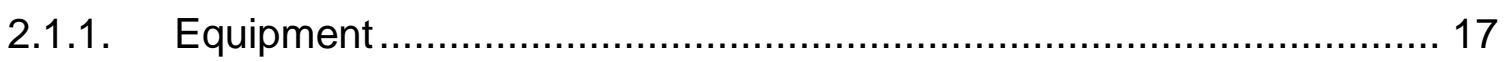

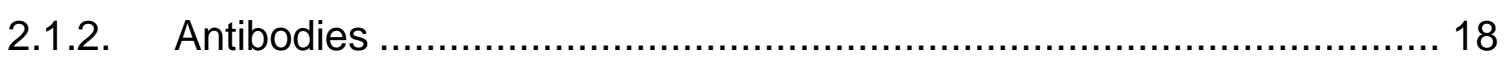




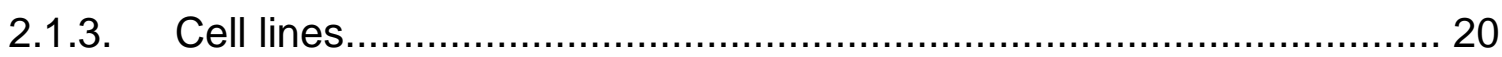

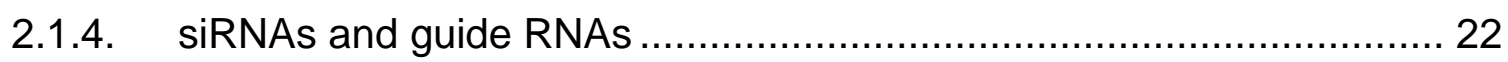

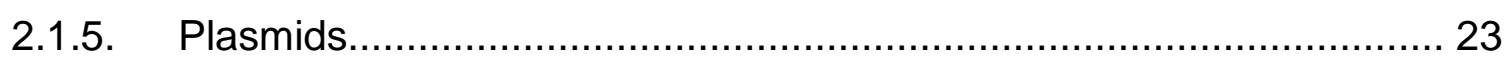

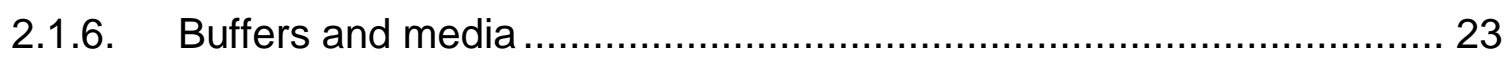

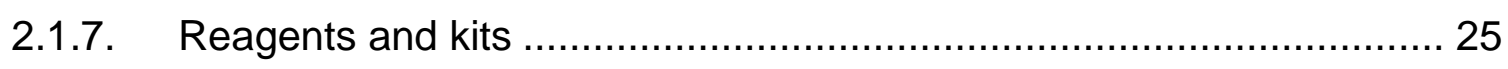

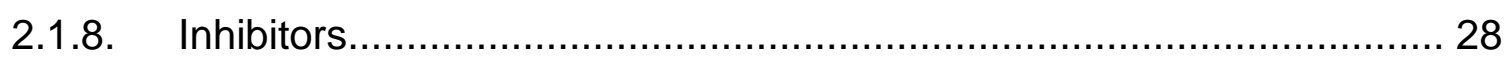

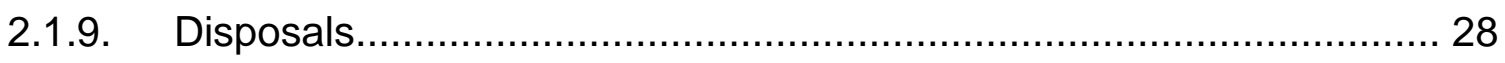

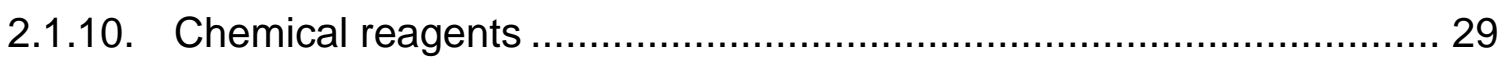

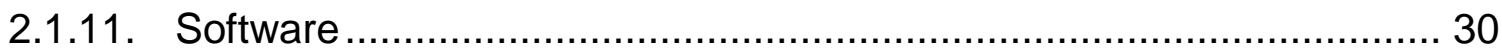

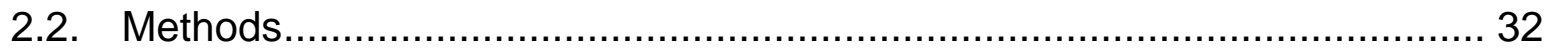

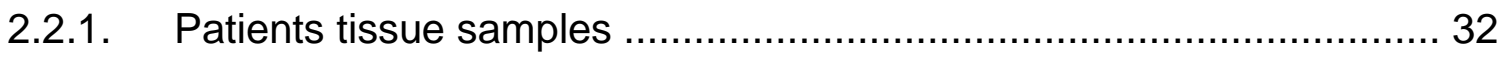

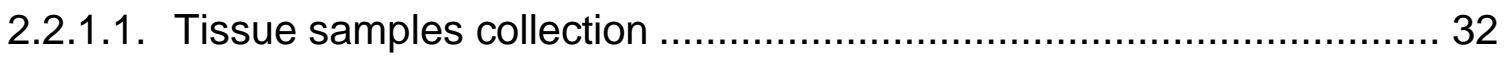

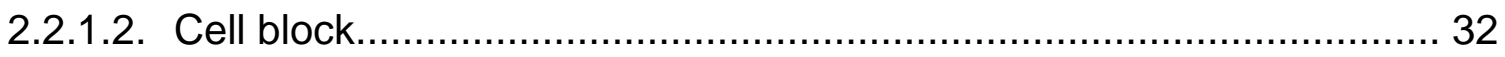

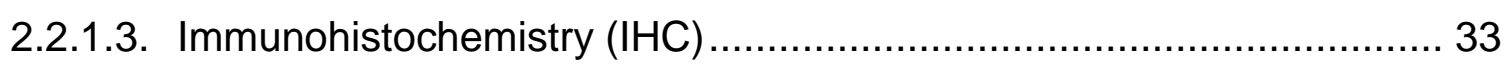

2.2.1.4. Florescence in situ hybridization (FISH) ................................. 33

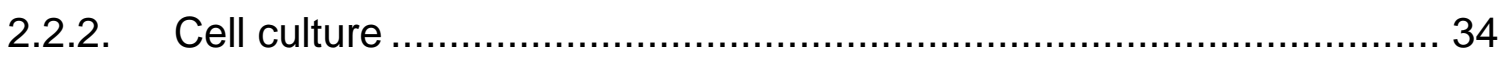

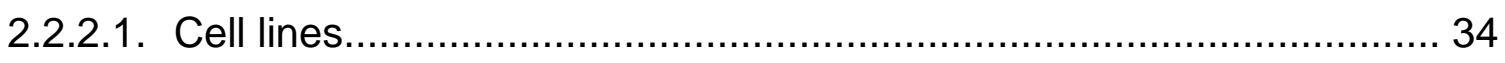

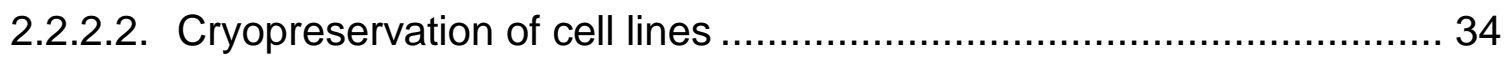

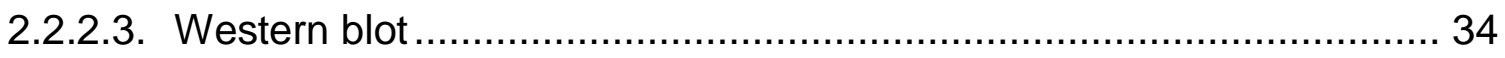

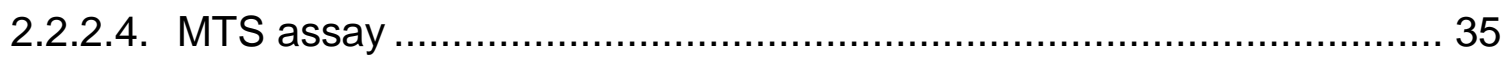

2.2.2.5. Cell counting and viability using Guava® Muse® cell analyzer ........... 35

2.2.2.6. PI viability assay ............................................................ 36

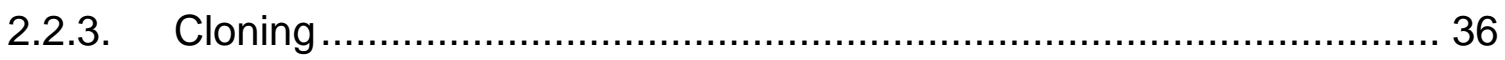

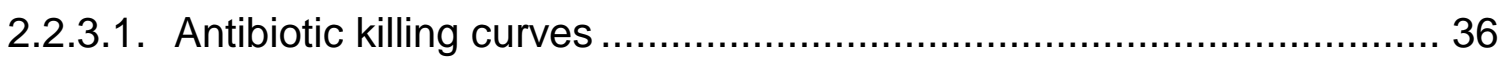

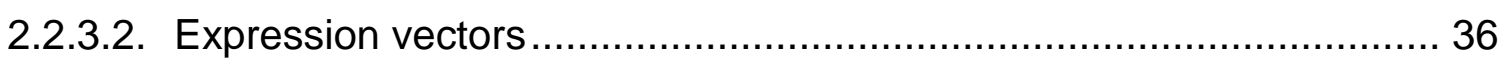

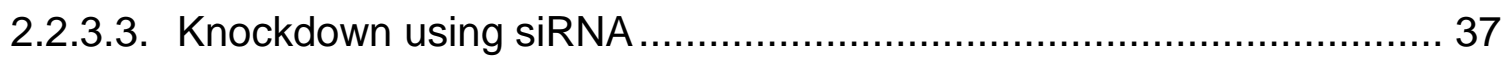

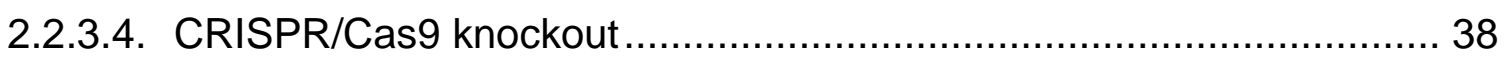

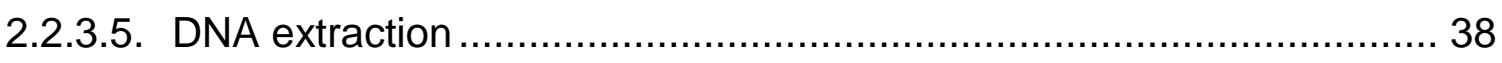


2.2.3.6. Sanger sequencing 39

2.2.3.7. Library preparation for Archer FusionPLEX CTL sequencing panel.... 39

2.2.4. Phosphoproteomic Mass spectrometry analysis ............................... 39

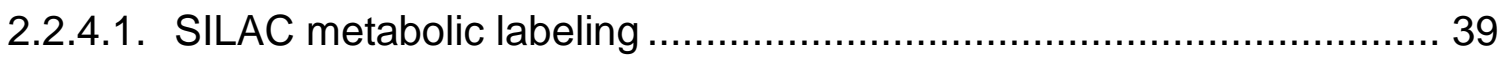

2.2.4.2. Lysing SILAC labelled cells ........................................................... 40

2.2.4.3. Mass spectrometry sample preparation .......................................... 40

2.2.4.4. Perseus phosphoproteomic data analysis ....................................... 41

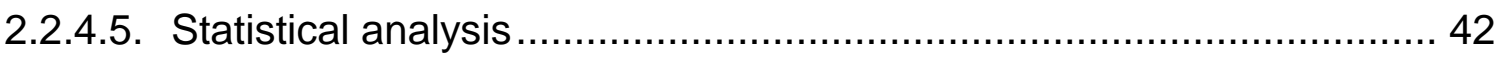

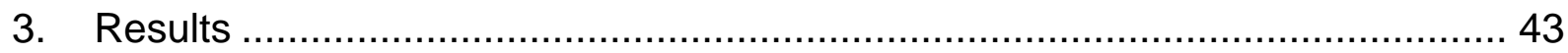

3.1. Studying prevalence and correlation of FGFR1 gene amplification and protein expression in lung cancer patients.

3.1.1. FGFR1 amplification as molecular target in squamous cell lung cancer 43

3.1.2. FGFR1 gene amplification in SQCLC and SCLC samples 44

3.1.3. Validation of immunostaining 46

3.1.4. FGFR1 protein expression in lung cancer 49

3.1.5. Correlation between FGFR1 gene amplification and protein expression in lung cancer 51

3.2. Studying mechanisms of resistance to FGFR1 inhibition in lung cancer .....53

3.2.1. Screening sensitivity to FGFR1 inhibition in human lung cancer cell lines 53

3.2.2. FGFR1 amplification in human lung cancer cell lines 54

3.2.3. Assigning cell line model to study FGFR1 inhibition resistance in lung cancer 55

3.2.4. FGFR1 protein expression in human lung cancer cell lines 56

3.2.5. Validation of sensitivity to FGFR1 inhibition in the cell line model....... 59

3.2.6. Testing AZD4547 (FGFR1 inhibitor) phosphorylation inhibition function 61

3.2.7. Testing AZD4547 (FGFR1 inhibitor) specificity using siRNA. 62 
3.2.8. Exploring activated FGFR1 signaling in the cell lines model...............65

3.2.9. Activation of Akt in FGFR1-amplified SQCLC tissue samples............ 67

3.2.10. Combination of FGFR1 and Akt inhibition ......................................... 69

3.2.11. Inducing Akt expression and activation in the sensitive cell lines........ 72

3.2.12. Inducing resistance to FGFR1 inhibition in sensitive cell lines ........... 76

3.2.13. Inducing resistance to FGFR1 inhibition in single clones of sensitive cell lines $\quad 77$

3.2.14. Combination inhibition of FGFR1 and Akt in resistant single clones ... 79 3.3. Phosphoproteomic analysis of FGFR1 inhibition resistance in lung cancer cell lines 85

3.3.1. Sample preparation for phosphoproteomic analysis ......................... 85

3.3.2. Phosphoproteomic LC-MS/MS data quality control............................ 87

3.3.3. Phosphoproteomic LC-MS/MS data bioinformatics functional analysis91

3.3.4. Functional validation of the proposed FGFR1 inhibition resistance pathway 98

4. Discussion 104

4.1. Molecular targets in lung cancer. 104

4.2. Studying prevalence of FGFR1 gene amplification in SQCLC and SCLC primary patient samples 105

4.3. Validating immunohistochemistry technique and antibody .................... 106

4.4. Studying prevalence of FGFR1 expression in AC, SQCLC and SCLC primary patient samples 107

4.5. Correlation between FGFR1 gene amplification and protein expression in lung cancer primary patient samples and cell lines 107

4.6. Building a lung cancer cell lines model to study mechanisms of FGFR1 inhibition resistance. 108

4.7. Validation of AZD4547 tyrosine kinase inhibition activity and specificity to FGFR1 109 
4.8. Activated signaling pathway in sensitive and resistant cell lines to FGFR1 inhibition

4.9. Activation of Akt in FGFR1 amplified and FGFR1-expressing primary SQCLC tissue samples

4.10. Combining FGFR1 and Akt inhibition to sensitize resistant cells to FGFR1 inhibition

4.11. Inducing Akt activation in sensitive lung cancer cell lines to FGFR1 inhibition 113

4.12. Induction of FGFR1 resistance in FGFR1 inhibition sensitive batch cell lines 114

4.13. Inducing FGFR1 resistance in single clones of FGFR1 inhibition sensitive cell lines 115

4.14. Sample preparation for LC-MS/MS phosphoproteomic analysis in FGFR1 inhibition resistant lung cancer cell lines

4.15. Quality control of LC-MS/MS phosphoproteomic analysis results

4.16. Activated signaling pathways among different models of FGFR1 inhibition resistant lung cancer cell lines

4.17. Validation of PAK1 and FAK as a molecular targets in intrinsic and induced FGFR1 resistant lung cancer cell lines.

4.18. Conclusion 120

4.19. Outlook

5. References 123

6. Curriculum vitae

Fehler! Textmarke nicht definiert. 


\section{List of tables}

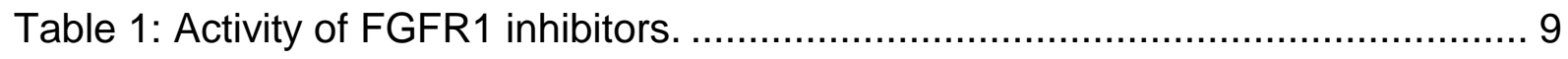

Table 2: Lung cancer patient characteristics. .............................................. 44

Table 3: FGFR1 gene amplification analyzed by fluorescence in situ hybridization (FISH) in multiple lung cancer cell lines.................................................... 55

Table 4: Combination inhibition interactions in $\mathrm{H} 520$ and $\mathrm{H} 1703$ based on Chou-

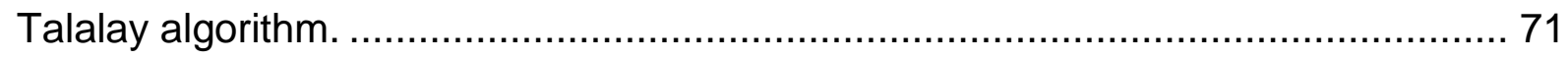

Table 5: Statistics of inducing resistance to the FGFR1 inhibitor AZD4547............ 78 


\section{List of figures}

Figure 1: Histological types of Lung Cancer. ..................................................... 2

Figure 2: Prevalence of potential driver mutations in lung cancer. ……………...... 6

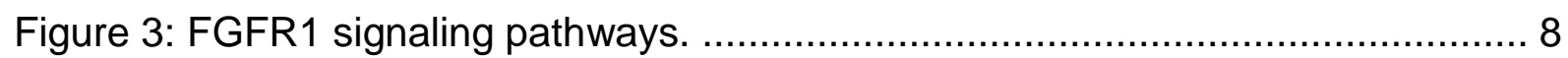

Figure 4 Summary of LC-MS/MS phosphoproteomic analysis sample preparation. . 15

Figure 5: FGFR1 gene amplification in tissue samples of lung cancer patients........ 45

Figure 6: Prevalence of FGFR1 gene amplification in lung cancer patient tissue samples.

Figure 7: Survival analysis according to FGFR1 amplification in lung cancer patients. 46

Figure 8: Validation of anti-FGFR1 antibody in human tissue samples. 47

Figure 9: CRISPR/Cas9 knockout of FGFR1 gene in $\mathrm{H} 1703$ lung cancer cell line... 48 Figure 10: Validation of anti-FGFR1 antibody using the FGFR1-knockout cell line.. 48 Figure 11: IHC staining of FGFR1 protein expression in SQCLC patient tissue samples.

Figure 12: FGFR1 protein expression in SQCLC, SCLC and AC patient tissue samples.

Figure 13: Correlation between FGFR1 protein expression and lung cancer patients' overall survival. 50

Figure 14: Correlation between FGFR1 gene amplification and protein expression. 52 Figure 15: Screening sensitivity of lung cancer cell lines to FGFR1 inhibition. 54

Figure 16: Prevalence of FGFR1 gene amplification (FISH analysis) in lung cancer cell lines. 56

Figure 17. FGFR1 protein expression in multiple lung cancer cell lines. 57

Figure 18: Immunocytochemical staining of FGFR1 protein in lung cancer cell lines. 58

Figure 19: Collective status of FGFR1 gene amplification and protein expression in lung cancer cell lines. 59

Figure 20: Sensitivity to the FGFR1 inhibitor AZD4547. 60

Figure 21: Sensitivity to the FGFR1 inhibitor BGJ398. 60

Figure 22: Cell growth assay (number of viable cells) after treatment with AZD4547.

Figure 23: Phosphorylation inhibition through AZD4547. 62 
Figure 24: Transfection efficiency of siRNAs in lung cancer cell lines. ...................63

Figure 25: siRNA knockdown of FGFR1 protein........................................... 63

Figure 26: Proliferation assay under FGFR1-siRNA knockdown. .........................6 65

Figure 27: Western blot analysis of FGFR1 signaling in control, resistant and

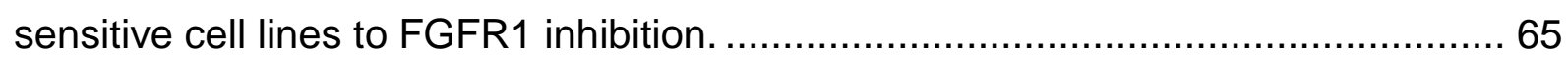

Figure 28: RNA-sequencing using Archer FusionPlex CTL Panel. ........................67

Figure 29: Immunohistochemical staining of pAkt in patient samples....................68

Figure 30: Expression levels of phosphorylated Akt in FGFR1-amplified and FGFR1-

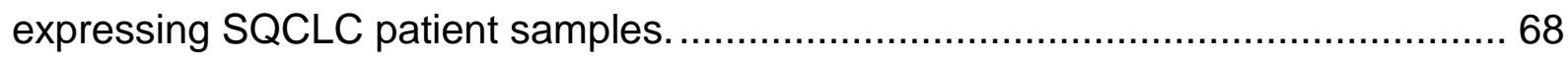

Figure 31: Survival analysis of FGFR1-amplified and FGFR1-expressing SQCLC patient samples.

Figure 32: Combination inhibition of FGFR1 and Akt in sensitive and insensitive cell lines FGFR1 inhibition.

Figure 33: Synergistic effect of FGFR1 and Akt co-inhibition in $\mathrm{H} 520$ and $\mathrm{H} 1703$ cell lines.

Figure 34: Immunoblotting of phosphorylated Akt in control and constitutively activeAkt cell lines

Figure 35. Sensitivity to FGFR1 inhibition in mutationally activated Akt cell lines..... 73 Figure 36: Combination inhibition of FGFR1 and Akt in control and MyrAkt1 expressing cell lines.

Figure 37: Combination index values in control and mutationally activated Akt cell lines. 75

Figure 38: Acquired resistance to FGFR1 inhibition in the three sensitive cell lines. 76 Figure 39: Western blot analysis of Akt phosphorylation levels in acquired resistant cell lines. 77

Figure 40: Western blot analysis showing altered signaling in FGFR1 inhibition resistant clones compared to parental controls. 79

Figure 41: Synergistic effect of FGFR1 and Akt co-inhibition in the FGFR1 inhibition resistant clone $\mathrm{H} 1581-11 \mathrm{R}$. 80

Figure 42: Combination index values indicated type of interaction between the FGFR1 inhibitor AZD4547 and the Akt inhibitor AZD5363 in H1581-11R resistant clone.

Figure 43: Effect of FGFR1 inhibition in parental and resistant clones of $\mathrm{H} 1581$ cell line. 82 
Figure 44: Synergistic effect of FGFR1 TKI combined with Akt inhibitor in resistant clones

Figure 45: Activated signaling pathway in induced resistance single clones of $\mathrm{H} 1581$ cell line.

Figure 46: RNA sequencing showed protein expression levels among resistant clones. 84

Figure 47: Design of SILAC metabolic labeling of cell lines. 86

Figure 48: Protein lysates assembly for mass spectrometric analysis. 86

Figure 49: Numeric Venn diagram showed total number of quantified phosphosites among different groups. Phosphosites were quantified among two biological replicates and two technical replicates in each of the groups.

Figure 50: Multiple scatter plot for all samples in global proteome phosphopeptides group. 88

Figure 51: Multiple scatter plot for all samples in phospho-tyrosine enriched group. 89 Figure 52: Normal distribution of values in global proteome group. 90

Figure 53: Normal distribution of values in phospho-tyrosine enriched group. 91

Figure 54: Heatmaps of significantly differentiated intensities of phosphosites among different GPome samples.

Figure 55: Heatmaps of significantly differentiated intensities of phosphosites among different pYome samples. 93

Figure 56: Volcano plots show significantly differentiated phosphosites. 94 Figure 57: Volcano plots of significantly differentiated phosphosites in pYome samples.

Figure 58: Fold change of significantly over and down phosphorylated targets upstream and downstream Akt in resistant cell lines compared to control H1581 cell line.

Figure 59: Proposed resistance pathway in FGFR1 inhibition resistant cell lines compared to $\mathrm{H} 1581$ control cell lines.

Figure 60: MTS assays show combination between PAK1 or FAK and FGFR1 inhibition in FGFR1 inhibition intrinsic resistant cell lines.

Figure 61 MTS assays show combination between PAK1 or FAK and FGFR1 inhibition in FGFR1 inhibition induced resistant cell lines. 100

Figure 62: Synergistic effect between FGFR1 and PAK1 or FAK co-inhibition in FGFR1 inhibition induced and intrinsic resistant cell lines. 101 
Figure 63: Immunoblotting for FGFR1 and FAK co-inhibition in resistant cells...... 102 Figure 64: Combination between FGFR1 inhibition and CD44 knockdown in FGFR1 inhibition resistant lung cancer cells. 103 


\section{Summary}

Lung cancer continuous to be the leading cause of cancer-related death worldwide. A relatively new and effective approach of treating lung cancer is achieved through targeting specific molecular alterations in lung cancer cells. While effective molecular therapies have been developed and approved in adenocarcinoma of the lung (AC), squamous cell lung cancer (SQCLC) and small cell lung cancer (SCLC) still lack any approved molecular targets.

Fibroblast growth factor receptor 1 (FGFR1) is a promising molecular target in squamous cell lung cancer and small cell lung cancer subtypes. Clinical trials proved the principle of targeting FGFR1 in SQCLC patients harboring FGFR1 amplification. However, modest response rates of patients treated with FGFR1 inhibitors suggested presence of different layers of resistance, which are either pre-existed in patients or developed over the course of treatment.

In this thesis, I used a cohort of 421 lung cancer primary patient samples to screen prevalence of FGFR1 gene amplification among SQCLC and SCLC groups using fluorescence in situ hybridization technique (FISH). FGFR1 protein expression was screened among AC, SQCLC and SCLC groups using immunohistochemistry (IHC). The antibody used in IHC analysis was validated through CRISPR-Cas9 knockout system. FGFR1 gene amplification prevalence was compared to protein expression in the same set of patients. In order to understand molecular mechanisms that underline FGFR1 inhibition resistance in lung cancer cells, I established a model of resistant cell lines and compared them to control sensitive cell lines. FGFR1 inhibition resistance model consisted of intrinsic resistant lung cancer cell lines, induced resistant lung cancer single clones and a mutationally resistant lung cancer cell line. Furthermore, mass spectrometric phosphoproteomic analysis was performed to compare FGFR1 inhibition resistant cell lines to a control cell line under control and inhibition status. MTS viability assay together with other cell viability assays were used to validate the co-inhibition effect of FGFR1 alongside with other potential molecular targets in FGFR1 resistant lung cancer cells.

FISH analysis revealed $23 \%$ and $8 \%$ prevalence of FGFR1 gene amplification among SQCLC and SCLC patients. FGFR1 protein was strongly expressed in $9 \%, 4 \%$ and $35 \%$ of SQCLC, SCLC and AC patients, respectively. Statistical analysis showed no 
correlation between FGFR1 gene amplification and protein expression in lung cancer patients. Comparing activated signaling pathways between FGFR1 inhibition sensitive and resistant cell lines showed Akt activation as a significant difference between the two groups. MTS combination inhibition assays confirmed synergy between FGFR1 inhibition and Akt inhibition in intrinsic, induced and constitutively active Akt resistant lung cancer cell lines. Mass spectrometric phosphoproteomic analysis showed a common resistance pathway in intrinsic and induced resistant cells to FGFR1 inhibition. Over phosphorylated CD44, FAK, PAK1, Paxillin and Afadin proteins formed the core of the resistance pathway. MTS viability assays combined with Chou-Talalay combination index analyses confirmed synergy between FGFR1 and PAK1 or FAK inhibition in intrinsic and induced resistant lung cancer cell lines to FGFR1 inhibition. Finally, CD44 knockdown combined with FGFR1 inhibition showed significant reduction in proliferation of resistant cells compared to single treatments.

To conclude, the current thesis confirmed previously published prevalence of FGFR1 gene amplification (23\% in SQCLC and $8 \%$ in SCLC) and protein expression ( $9 \%$ in SQCLC, $4 \%$ in SCLC and $35 \%$ in AC) in lung cancer patients. The thesis revealed that FGFR1 gene amplification seems not to be the most accurate selection criteria of patients who will benefit from FGFR1 inhibition therapy. Molecular and phosphoproteomic analyses alongside with functional validation assays suggested that CD44, PAK1, FAK and Akt are promising potential targets that could synergistically sensitize resistant lung cancer patients to FGFR1 inhibition. 


\begin{tabular}{|c|c|}
\hline \multicolumn{2}{|c|}{ Abbreviations } \\
\hline$\%$ & Percent \\
\hline${ }^{\circ} \mathrm{C}$ & Degrees Celsius \\
\hline A & Purine base Adenin \\
\hline$A b$ & Antibody/antibodies \\
\hline$A C$ & Adeno carcinoma \\
\hline Akt & Protein kinase $\mathrm{B}$ \\
\hline ALK & Anaplastic Lymphoma Kinase \\
\hline bp & Base pair \\
\hline BSA & Bovine serum albumin \\
\hline BSA & Bovine serum albumin \\
\hline C & Pyrimidine base Cytosine \\
\hline CEN8 & Centromere 8 \\
\hline $\mathrm{CO} 2$ & Carbon dioxide \\
\hline CTC & Circulating tumor cells \\
\hline $\mathrm{Da}$ & Dalton \\
\hline DAPI & 4',6-diamidino-2-phenylindole \\
\hline $\mathrm{ddH} 2 \mathrm{O}$ & Bi-distilled H2O \\
\hline DMEM & Dulbecco's Modified Eagle Medium \\
\hline DMSO & Dimethyl sulfoxide \\
\hline DNA & Deoxyribonucleic acid \\
\hline dNTP & Deoxyribonucleotide \\
\hline e.g. & Exempli gratia \\
\hline EDTA & Ethylene diamine tetraacetic acid \\
\hline EGF & EGFR Epidermal growth factor receptor \\
\hline EGFR & Epidermal growth factor receptor 1 \\
\hline ERK & Extracellular signal regulated kinases \\
\hline et al. & et alteri (and others) \\
\hline $\mathrm{EtOH}$ & Ethanol \\
\hline FACS & Fluorescence-Activated Cell Sorting \\
\hline FBS & Fetal bovine serum \\
\hline FCS & Fetal calf serum \\
\hline
\end{tabular}


FDA Food and drug administration

FGF Fibroblast growth factor

FGFR1 fibroblast growth factor receptor 1

FISH Fluorescence in situ hybridization

Fw Forward

G Purine base Guanine

g Relative centrifugal force

g Gram

GFP Green-fluorescent protein

GPome Serine and threonine phosphorylated peptides

gRNA Guide RNA

$\mathrm{H} 2 \mathrm{O} 2$ Hydroxic peroxide

$\mathrm{HCl} \quad$ Hydrochloric acid

HPLC High performance liquid chromatography

i.e. Id est

IASLC International Association for the Study of Lung Cancer

ICC Immunocytochemistry

Ig Immunoglobulin

IHC Immunohistochemistry

$\mathrm{KCl} \quad$ Potassium chloride

$\mathrm{kDa} \quad$ Kilo Dalton

L Liter

LC Lung cancer

LC Liquid chromatography

m Milli

M Molar

$\mathrm{m} / \mathrm{z} \quad$ Thomson value

mAb Monoclonal antibody

MALDI matrix-assisted laser desorption

MAPK Mitogen-activated protein kinase

MEK Mitogen activated protein kinase kinase

$\mathrm{MeOH} \quad$ Methanol 


\begin{tabular}{|c|c|}
\hline $\mathrm{mg}$ & Milligram \\
\hline Mg2+ & Magnesium \\
\hline $\min$ & Minute(s) \\
\hline $\mathrm{ml}$ & Milliliter \\
\hline $\mathrm{mM}$ & Millimolar \\
\hline MPI & Max Planck Institute \\
\hline MS & Mass spectrometry \\
\hline $\mathrm{NaCl}$ & Sodium chloride \\
\hline $\mathrm{NaOH}$ & Sodium hydroxide \\
\hline NEM & $\mathrm{N}$-ethylmaleimide \\
\hline $\mathrm{ng}$ & Nanogram \\
\hline NGS & Next generation sequencing \\
\hline $\mathrm{NIH}$ & National institute of health \\
\hline $\mathrm{nM}$ & Nanomolar \\
\hline NP-40 & Nonidet P40 \\
\hline $\mathrm{O} 2$ & Oxygen \\
\hline $\mathrm{oC}$ & Degree Celsius \\
\hline$p$ & Phospho \\
\hline $\mathrm{P} / \mathrm{S}$ & Penicillin/streptomycin \\
\hline p53 & Tumor protein 53 \\
\hline PARK7 & Parkinsonism Associated Deglycase \\
\hline PBS & Phosphate buffered saline \\
\hline PCR & Polymerase chain reaction \\
\hline $\mathrm{pH}$ & Preponderance of hydrogen ions \\
\hline $\mathrm{PI}$ & Propidium iodide \\
\hline $\mathrm{PI3K}$ & Phosphatidylinositol-4,5-bisphosphate 3-kinase \\
\hline PLC & Phospholipase C \\
\hline Pyome & Tyrosine phosphorylated peptides \\
\hline RNA & Ribonucleic acid \\
\hline RPM & Rotations per minute \\
\hline RPMI & Roswell Park Memorial Institute \\
\hline RT & Room temperature \\
\hline
\end{tabular}


RTK Receptor tyrosine kinases

$\mathrm{Rv}$

Reverse

SCLC

Small cell lung cancer

SCX

Strong cation exchange

SD

Standard deviation

SDS

Sodium dodecyl sulfate

$\sec$

Second

Seq

Sequencing

$\mathrm{SH} 2 \quad$ Src-homology-2 domains

SILAC Stable isotope labeling by amino acids in cell culture

siRNA Small interfering RNA

SQCLC Squamous cell lung cancer

STAT signal transducer and activator of transcription

Pyrimidine base Thymidine

Taq

Thermus aquaticus

TBE

Tris/borate/EDTA

TKIs tyrosine kinase inhibitors

TNM Tumor, node and metastasis system

Tris Tris(hydroxymethyl)aminomethane

U Units

UMG University Medical Center Goettingen

UMG University medical center of Goettingen

WHO World health organisation

$\mu \quad$ Micro

$\mu \mathrm{g} \quad$ Microgram

ul Microliter 


\section{Introduction}

\subsection{Lung cancer}

\subsubsection{Prevalence and Mortality}

Lung cancer is the primary cause of cancer-related death worldwide. According to the World Health Organization (WHO), 2.1 million cases were newly diagnosed in 2018 (IARC, 2018). In the same year, lung cancer has killed 1.8 million patients, which constituted $18.4 \%$ of the total cancer mortality (IARC, 2018). Lung cancer has the highest incidence in both sexes compared to other tumors. However, males have higher incidence and mortality rate than females. The peak of incidence and mortality is at age range of 60 to 75 years in both sexes (Kozielski et al., 2012; Torre et al., 2016). Bad prognosis and low five-year survival rate are known characteristics of this tumor. Survival rates are substantially higher with early diagnosis. In USA, the fiveyear survival rate of early stage diagnosis lung cancer is $56 \%$, while this percentage falls down to $2-5 \%$ in late stage lung cancer (Bozinovski et al., 2016; NIH, 2018).

Various risk factors have been correlated to lung cancer. Tobacco smoking forms the most significant and common risk factor. Long time cigarette smoking decreases life expectancy by about ten years (Bruske-Hohlfeld, 2009). Exposure to second hand smoking, asbestos silica in industry, arsenic in water, radioactive radon gas and some identified susceptibility genes are further validated risk factors (BruskeHohlfeld, 2009; Mao et al., 2016).

\subsubsection{Histological classification}

Lung cancer classification is dynamically improving and updating every few years by virtue of continuous research and discovery of new biomarkers that render diagnosis and staging of the tumor more specific and allow for more precise treatment. Histologically, lung cancer is classified into small cell lung cancer (SCLC, $\sim 15 \%$ of cases) and non-small cell lung cancer (NSCLC, $~ 85 \%$ of cases) (Figure 1). Nonsmall cell lung cancer is further divided into Adenocarcinoma (AC) and squamous cell lung cancer (SQCLC) (Woodard et al., 2016). Adenocarcinoma of the lung is the most common NSCLC and is characterized by its tubular or acinar structure and potential production of mucin. AC usually stains for TTF-1, CK7 and/or Napsin A in immunohistochemistry (IHC) (Woodard et al., 2016). Squamous cell lung cancer is 
characterized by intercellular bridges and potential keratinization. Common markers for SQCLC are P40, CK5/6 and P63. Squamous cell lung cancer is divided into keratinizing, non-keratinizing and basaloid SQCLC (Inamura, 2017; Zheng, 2016).

Staging of lung cancer is a crucial part of diagnosis. It has a direct effect on prognosis as well as treatment strategies. According to the International Association for the Study of Lung Cancer (IASLC) and the Tumor, Node and Metastasis system (TNM), lung cancer is divided into four main stages based on tumor size and spread (Woodard et al., 2016). Stage one is assigned to small tumor sizes below five centimeters with no nodal or distal metastasis. Stage two includes tumors between three and seven centimeters that can be accompanied by lymph node metastasis but without distal metastasis (Sica \& Gal, 2012). Stage three tumors are usually larger than seven centimeters with mediastinal or subcarinal lymph node metastasis. The last and most aggressive stage is number four where the tumor can have different sizes at the lung alongside with distal metastasis (Sica \& Gal, 2012; Woodard et al., 2016).

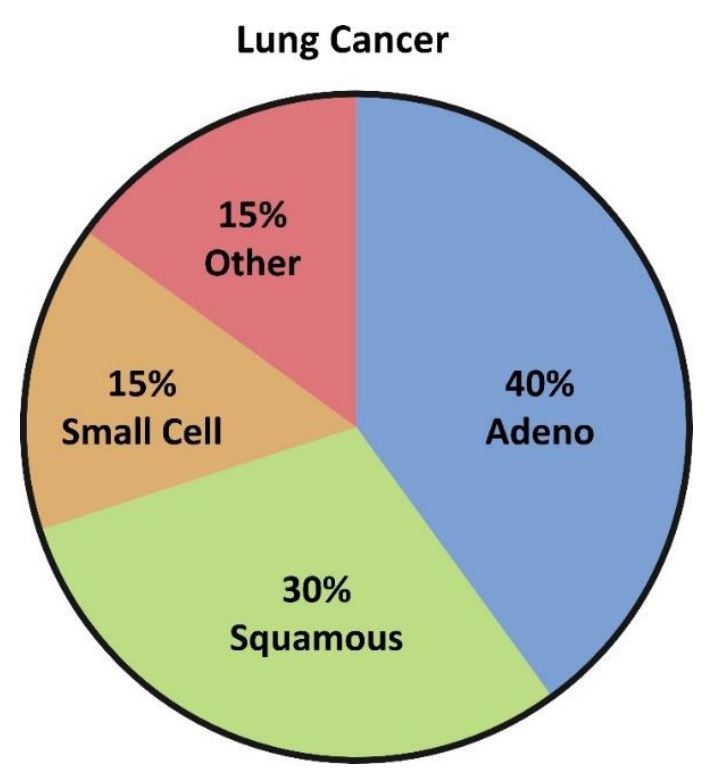

Figure 1: Histological types of Lung Cancer.

\subsubsection{Squamous cell lung cancer (SQCLC)}

Squamous cell lung cancer is the second most frequent histological type of non-small cell lung cancers, which forms about 30\% of lung cancer (Figure 1) (Bozinovski et al., 2016). Mortality of SQCLC is one of the highest in lung cancer with 400,000 cases worldwide (Gandara et al., 2015). Smoking associated SQCLC used to be the 
most frequent NSCLC. This dominance changed when cigarette companies started to use filters in their cigarettes. Filters allowed only smaller particles of carcinogens to be inhaled, which lead to a rise in adenocarcinoma incidents. However, smoking in its direct and indirect forms still makes up 90\% of SQCLC cases (Gandara et al., 2015). Finally, the spread of awareness about smoking risks decreased SQCLC incidents (Gandara et al., 2015).

SQCLC is usually diagnosed at late stages (stage three or four) due to lack of early symptoms. Late diagnosis eliminates choice of surgical intervention or focused radiotherapy, which are the treatments of choice for early stages. Patients diagnosed with SQCLC, like NSCLC in general, are usually treated with systematic chemotherapy. These treatment options can explain the poor five-year survival rate of NSCLC of about $2 \%$ (Bozinovski et al., 2016).

\subsection{Targeted therapies in lung cancer}

\subsubsection{Significance of molecular targets}

Conventional systemic treatments like chemotherapy have been shown to be toxic and in some cases tumorigenic in themselves (X. Chen et al., 2017). Toxic off-target effects and acquired resistance to conventional therapy led to the urge of developing new treatments, which are safer and more specific. Over the years, continuous cancer research shed the light on the molecular mechanisms underlying tumorigenesis in different tumor entities and sub-types. Heterogeneity of cancer cells is now well described in different types of tumors. Differences in molecular mechanisms of tumor cells can explain the wide variation in prognosis, response, resistance and relapse found in patients diagnosed and treated in the same way. Understanding specific molecular mechanisms of a certain cancer type allows not only for its accurate diagnosis but also for identifying specific molecular targets against it. Molecular targets are on the one hand specific for tumor cells and on the other hand vital for tumor growth, survival and progression. Molecular targets in cancer therapy can take the form of genes, proteins or components of tumor microenvironment (Kummar \& Doroshow, 2013). Expression and activation levels of these specific targets are usually altered in tumor tissues compared to healthy cells, which frequently stand behind tumorigenesis initiation, progression and spread. 
Under this new category of therapy, patients are not only diagnosed for tumor type but also for activation/deactivation of specific targets and biomarkers.

Identification of specific biomarkers or molecular targets is the first step towards achieving successful personalized cancer therapy. Biomarkers are usually identified through comparing cancer cells to normal cells on different levels. DNA sequencing of tumor cells can identify mutated genes, which express mutant proteins that could be essential in tumor growth and survival. BRAF mutation is an example of a mutant protein (RAF kinase) that leads to overactivation of growth signaling pathways and subsequently mutagenesis. Vemurafenib (Zelboraf $\AA$ ) is an approved small molecule that can inhibit mutated BRAF, e.g., in melanoma patients (Bollag et al., 2012; NIH, 2019).

Discovery of new tumor biomarkers could be based on proteomic screenings. Differential analysis of expressed proteins between tumor and healthy cells can lead to identification of uniquely overexpressed proteins in tumor cells that are vital for their progression. These proteins can then be targeted by monoclonal antibodies. Trastuzumab or Herceptin ${ }^{\circledR}$ is the pioneer monoclonal antibody (mAb) and targeted therapy in general to be discovered and approved by the US Food and Drug Administration (FDA) in 1998. The pioneer antibody is an effective therapy for breast cancer in which it binds to Her2 extracellular domain leading to its blockage and inhibition of downstream signaling ( $\mathrm{NIH}, 2019$; Wilson et al., 2017).

Detecting chromosomal abnormalities is another method of identifying tumor-specific molecular targets. In some cases, chromosomal abnormalities lead to oncogenic fusion genes and proteins that can be targeted. Gleevec $\AA$ is an approved protein kinase inhibitor, which inhibits BCR-ABL fusion protein in some myeloid leukemia and gastrointestinal tumor patients (NIH, 2019; Somlyai et al., 2017; Van den Abbeele et al., 2003).

\subsubsection{Receptor tyrosine kinases (RTKs)}

The group of receptor tyrosine kinases (RTKs) consists of 58 receptors that regulate vital process in healthy and diseased cells through phosphorylating downstream targets. Composition of receptor protein kinases is usually similar in terms of having three main domains: extracellular, transmembrane and intracellular domains. 
Extracellular domain or $\mathrm{N}$ terminal of the receptor is where specific growth factors bind to their corresponding receptors. Binding of growth factors initiates receptor activation and two receptor monomers start to dimerize/oligomerize. Dimerization process starts autophosphorylation and releases autoinhibition of the intracellular kinase domain ( $C$ terminal). For most RTKs, monomer receptors are an inactive form, while dimers are an active form. However, some receptors have a continuous shift between inactive monomers and inactive dimers, which are activated and stabilized only through ligand binding. (Du \& Lovly, 2018)

In healthy cells, RTKS regulate key processes like cell proliferation, differentiation, angiogenesis, tissue repair and survival. Because of this vital role, dysregulation or abnormal activity of RTKs is usually involved in pathogenesis of various conditions like bone disorders, diabetes and arteriosclerosis (Lemmon \& Schlessinger, 2010). In tumorigenesis, RTKs are proven to play important roles in tumor initiation and progression in the lung, breast, colon and liver (Regad, 2015). Hence, RTKs are considered promising molecular targets in cancer therapy.

\subsubsection{Targeted therapy in non-small cell lung cancer (NSCLC)}

Late stage NSCLC, where tumor starts to spread into lymph nodes, other parts of the lung or distal organs, is routinely treated with systemic chemotherapy. This strategy of treatment has lasted for many years without improvement neither in technique nor in prognosis of the patients. Discovery and approval of effective targeted drugs in tumors like breast cancer and leukemia have led to the urge of discovering molecular targets in lung cancer. Adenocarcinoma of the lung was the first histological type of lung cancer to achieve successful targeted therapy. Epidermal growth factor (EGFR) mutations and Anaplastic Lymphoma Kinase (ALK) gene rearrangements are among specific molecular targets in adenocarcinoma. Drugs like gefitinib/erlotinib and alectinib/brigatinib that target EGFR mutations and ALK rearrangements, respectively, are usually prescribed in advanced NSCLC. Those target specific drugs have been successful in improving patients' overall survival (Figure 2) (Bethune et al., 2010; Lin et al., 2018).

Unlike adenocarcinoma, there are no approved target specific drugs in squamous cell lung cancer. This contradiction reflects on the one hand the complexity and heterogeneity of SQCLC, but on the other hand it shows the need of more research 
and efforts to find targetable specific biomarkers. Different genomic abnormalities have been described in SQCLC (Perez-Moreno et al., 2012). Phosphoinisitide 3kinase $\mathrm{C}(\mathrm{PI} 3 \mathrm{KC})$ mutation is the most common potentially targetable genetic alteration in SQCLC with prevalence of $33 \%$ followed by fibroblast growth factor receptor 1 (FGFR1) gene amplification which occurs in $22 \%$ of patients (PerezMoreno et al., 2012). However, these abnormalities should be further studied and understood to estimate the extent of their addictive roles in tumor growth and survival (Perez-Moreno et al., 2012; Schultheis et al., 2014; Sholl et al., 2015; L. P. Zhang et al., 2015).

Adenocarcinoma

SQCLC

SCLC

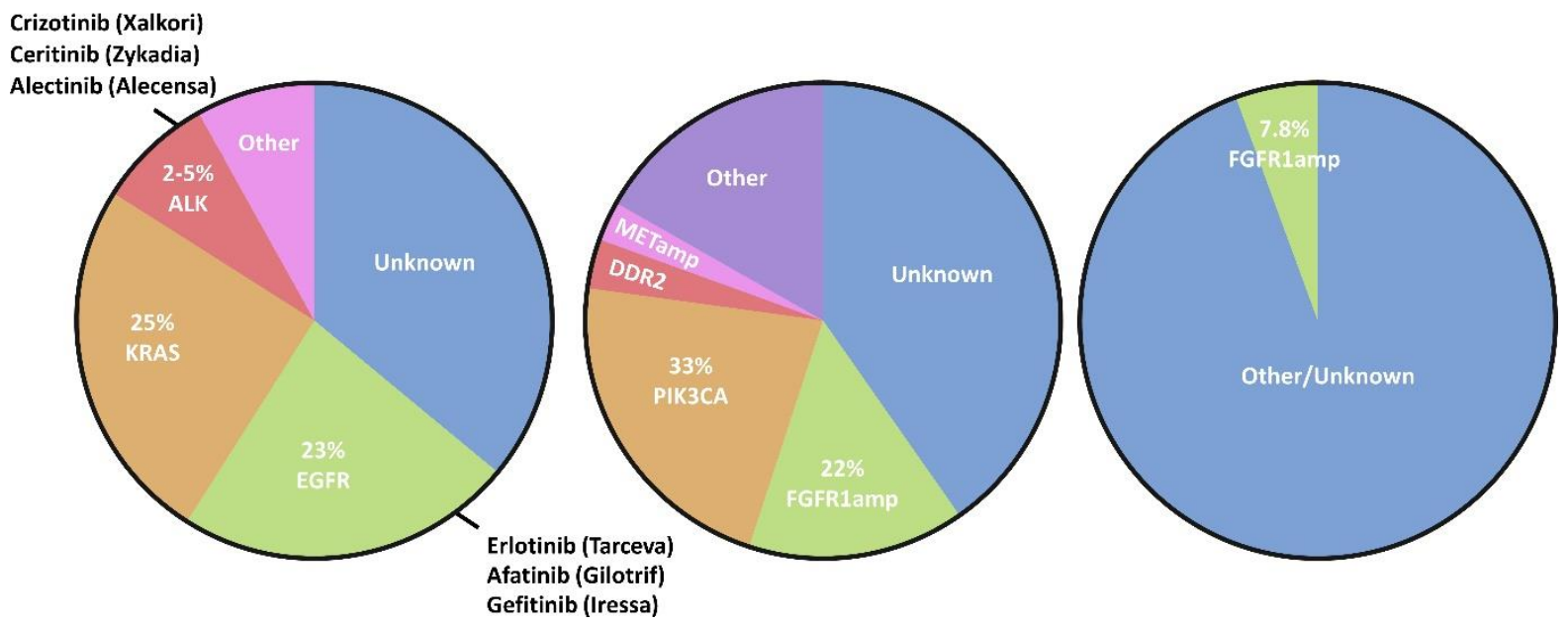

Figure 2: Prevalence of potential driver mutations in lung cancer.

\subsubsection{Fibroblast growth factor receptor 1 (FGFR1)}

Fibroblast growth factor receptor 1 (FGFR1) is a member of an RTK family, which consists of four receptors (FGFR1-4) and 22 ligands (FGF1-22). Three immunoglobulin-like domains (D1-3) build the extracellular part of the receptor. The extracellular domain contains an eight-residue protein (acid box), which binds to D1 and D2 and is involved in autoinhibition of the receptor. The intracellular part of the receptor starts with the juxta-membrane domain followed by two kinase domains on each arm of the receptor (Bae et al., 2009). Activation of FGFR1 and FGFRs in general, is slightly different from other RTKs. FGFRs require formation of stable bonds between three main players: ligand, receptor and heparin (Bae et al., 2009). 
Dimerization process starts by detachment of the acid box in order to increase affinity of receptors to fibroblast growth factors (FGFs) or heparin. Following removal of autoinhibition, dimerization of receptor occurs through binding of a bivalent ligand at D2 and D3 domains, binding of heparin sulfate and receptor-receptor interaction (Du \& Lovly, 2018). FGFRs dimerization starts the transformation of the intracellular tyrosine domain from an inactive cis-configuration to an active trans-configuration. Activated tyrosine domains recruit their downstream targets through their srchomology-2 domains (SH2) or their phosphotyrosine binding domains (Bae et al., 2009). Following activation of kinase domains, four main pathways are activated in FGFR1 signaling and are responsible for its role in cell growth, maintenance and survival. These pathways are RAS/RAF/MEK/MAPK pathway, PI3K/Akt pathway, JAK-STAT pathway and PLCY pathway (Figure 1.3) (Ornitz \& Itoh, 2015).

Dysregulation of FGFR1 signaling can be attributed to different factors. Three main genetic abnormalities do lead to overactivation of FGFR1 signaling. Firstly, amplification of the gene copy number, which is usually diagnosed by fluorescence in situ hybridization (FISH) can lead to protein overexpression and overactivation of downstream signaling pathways. Secondly, mutations at extracellular or intracellular domains of the receptor can lead to autoactivation and dimerization of the receptor without FGFs binding. Finally, genomic abnormalities like FGFR1 gene fusions can dimerize and activate the tyrosine kinase domains through binding to the fused proteins instead of normal growth factors. Other factors like alterations in noncoding regions, transcriptional factor or elevated levels of FGFs in tumor microenvironment can also lead to FGFR1 overactivation. (Katoh, 2019; Ornitz \& Itoh, 2015) 


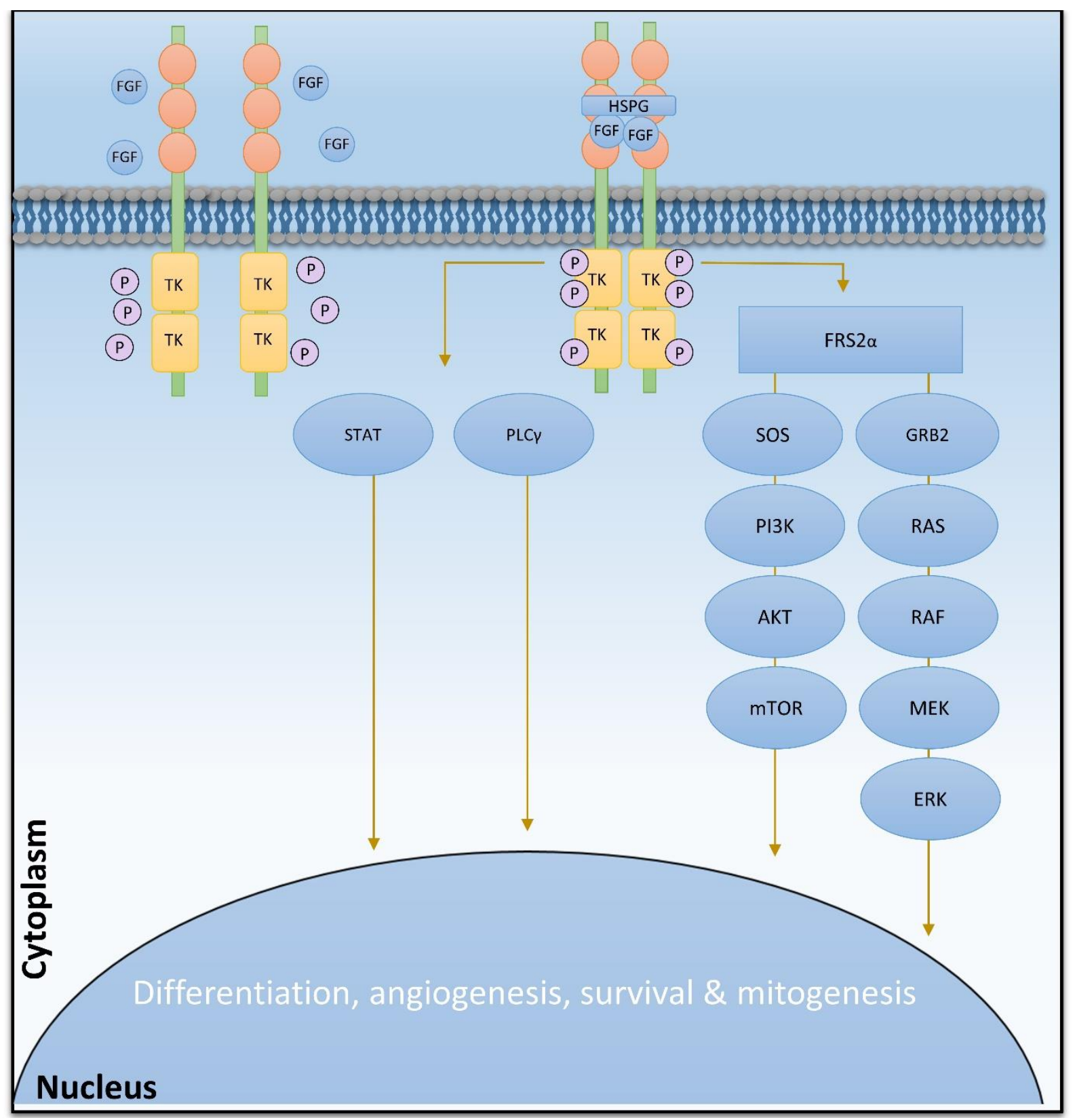

Figure 3: FGFR1 signaling pathways.

\subsection{Targeting FGFR1 in squamous cell lung cancer (SQCLC)}

Systemic chemotherapies are cytotoxic to fast proliferating cells in the body including tumor cells and healthy cells as well (Gronberg et al., 2010; Mittra et al., 2017). Apart from their broad activity and side effects, chemotherapies have shown low efficacy in advanced cases of NSCLC especially in SQCLC with a median overall survival of 9 to 11 months (Sholl et al., 2015; Socinski et al., 2012). The aforementioned reasons demonstrated the importance of developing targeted therapies for late stage lung cancer patients in general and SQCLC patients in particular. As outlined above, adenocarcinoma of the lung has been a successful example of how targeted drugs 
can lead to dramatic improvement of prognosis even in late stage lung cancer cases. Unlike adenocarcinoma, SQCLC lags behind without any approved targeted therapy (Tao et al., 2016; Y. C. Zhang et al., 2016).

Fibroblast growth factor 1 gene is amplified in about 22\% of SQCLC patients (Elakad et al., 2020; Sabari \& Paik, 2017; Sholl et al., 2015). Wide spread of FGFR1 amplification in SQCLC made it one of the most interesting and studied molecular targets in this group (Chae et al., 2017). So far, 19 small molecules tyrosine kinase inhibitors (TKIs) that can target and inhibit FGFR1 alongside with other RTKs have been developed. Out of these inhibitors, only three inhibitors are specific to FGFR1, which are AZD4547 developed by Astrazeneca@), Infigratinib (BGJ398) developed by Novartis@ and Debio1347 developed by Debiopharm@ (Katoh, 2019).

The tyrosine kinase inhibitors AZD4547 and BGJ398 are found to be highly specific FGFR1 inhibitors with IC50 values of less than one nanomolar (Table 1) (Katoh, 2019). Both of AZD4547 and BGJ398 TKIs have reached phase II clinical trials (Katoh, 2019). AZD4547 is considered the most specific second-generation FGFR1 inhibitor with 1000-fold more potency against FGFR1 than FGFR4. The Astrazeneca@ inhibitor is a type I inhibitor which binds and blocks the ATP site at the kinase domain in the active configuration of the receptor (Tucker et al., 2014).

Table 1: Activity of FGFR1 inhibitors.

\begin{tabular}{lcc}
\hline Target & $\begin{array}{c}\text { AZD4547 } \\
\text { IC50 (nM) }\end{array}$ & $\begin{array}{c}\text { BGJ398 } \\
\text { IC50 (nM) }\end{array}$ \\
\hline Approval & In clinical trials & In clinical trials \\
FGFR1 & $<1$ & 1 \\
FGFR2 & 3 & 1 \\
FGFR3 & 2 & 1 \\
FGFR4 & 165 & 60 \\
VEGFR2 & 24 & 180 \\
Others $<10 \mathrm{nM}$ & - & - \\
\hline
\end{tabular}

Phase II clinical trials of AZD4547 in SQCLC patients with FGFR1 amplification have shown partial response in 7-8\% of patients (Aggarwal et al., 2019; Paik et al., 2017). 
On the other side, BGJ398 has shown 11\% partial response in SQCLC with FGFR1 amplification patients during clinical trials (Nogova et al., 2017). In both studies, a group of patients, which formed around 7\% has shown very good response with significant raise in overall survival. These results have proven the concept of using FGFR1 as a molecular target in SQCLC patients. However, the relatively low response rate in clinical trials alongside with tumor relapse after initial response, revealed a common behavior of resistance against FGFR1 inhibitors (Paik et al., 2017).

Studies have attempted to understand reasons and mechanisms of resistance against FGFR1 inhibition. A possible reason for resistance could be co-amplified genes, which are located on the same region of chromosome 8 like ZNF708 and RAB11FIP1 genes (Kwek et al., 2009). Another reason, which could result in bad response to FGFR1 inhibition is the inconsistent correlation between FGFR1 gene amplification and protein expression levels in lung cancer patients (Elakad et al., 2020; Seo et al., 2014; von Massenhausen et al., 2013; Wynes et al., 2014). However, the most studied mechanism of resistance to FGFR1 inhibitors is the activation of a bypath signaling pathway/pathways that compensate for the inhibited pathway. Bypath resistance pathways could originate from previously activated pathways (intrinsic resistance) or newly activated pathways due to long exposure to the inhibitors (acquired resistance) (Nogova et al., 2017; Paik et al., 2017).

Current studies focus on understanding molecular mechanisms underlying both types of resistance (intrinsic and acquired) in order to find targets that can sensitize cells to FGFR1 inhibition. In 2016, Kim and colleagues have shown that Met kinase was overexpressed in a resistant clone developed from a sensitive cell line to FGFR1 inhibitors. They have concluded that combining FGFR1 inhibitors with Met inhibitors can re-sensitize resistant cells (S. M. Kim et al., 2016). In 2017, Lin and colleagues have found that c-Myc degradation was an essential step for FGFR/MEK-ERK pathway inhibition. Disruption or mutation of c-Myc can result in resistance to FGFR1 inhibitors in lung cancer cell lines ( $\mathrm{H}$. Liu et al., 2017). NRAS amplification has been shown to be able to reactivate inhibited FGFR1/MAPK pathway in $\mathrm{H} 1581$ lung cancer cell line (Malchers et al., 2017).

Multiple studies tried to understand mechanisms of resistance to FGFR1 inhibition in squamous cell lung cancer (Malchers et al., 2017; Singleton et al., 2015). However, 
the small size of models used in these studies, usually two or three cell lines, renders the findings very specific to the cell lines used and not to the general FGFR1 inhibition resistance mechanisms (Bockorny et al., 2018; Fumarola et al., 2017; Malchers et al., 2017). The aforementioned reason can also explain the versatile, and frequently contradictory findings of FGFR1 inhibition resistance in lung cancer cells. Literature studies have not compared intrinsic to acquired resistance of FGFR1 inhibition in lung cancer. Finally, until now, no phosphoproteomic or global proteomic analysis has compared sensitive and resistant cells to FGFR1 inhibition in lung cancer. Consequently, more efforts should be exerted in this direction in order to understand the exact mechanisms of resistance and to find effective targets that sensitize resistant SQCLC cells to FGFR1 inhibition.

\subsection{Proteomic analysis}

\subsubsection{Proteomic analysis in cancer research}

Cancer is a highly heterogenic disease that requires continuous invention and upgrading of high-resolution analytical tools to study. New analytical methods give us clearer, sometimes completely new, view on deep mechanisms of tumorigenesis. Over the years, genomic analysis through DNA sequencing was the standard tool to screen differences between healthy and cancer cells (Craig et al., 2016; Kamps et al., 2017). DNA sequencing is a robust method to screen genomic mutations; however, aberrant protein expression and signaling pathways overactivation can trigger tumorigenesis, too (Kosti et al., 2016; Raina et al., 2011). Measuring levels of genes end products gives us new insights about direct effectors of cell signaling and hence tumorigenesis (He et al., 2012; Panis et al., 2019). Applying proteomic screening techniques in cancer research is anticipated to help us finding new and accurate tumor biomarkers and prognostic factors for cancer treatment (Cho, 2017; Shruthi et al., 2016). In 2019, Suhas Vasaiker and his colleagues have presented a large scale proteogenomic study conducted on 110 human colon cancer patient samples compared to healthy tissue samples. The study has revealed the significance of proteomic analyses, particularly phosphoproteomic analyses, in not only validating and correcting genetically identified molecular targets but also identifying new biomarkers for cancer therapy (Vasaikar et al., 2019). 
The necessity of robust tools for large-scale proteomic analysis has ignited the development and improvements in mass spectrometry (MS) (Tyers \& Mann, 2003; Z. Zhang et al., 2014).

Mass spectrometry is the most powerful and robust tool available to analyze proteins from different biological samples with high throughput, low cost and high reproducibility (Nilsson et al., 2010). Mass Spectrometry can not only identify and quantify proteins, but also identify their interaction and posttranslational modification (Cho, 2017). Over the past few years, mass spectrometry was prone to extensive research and development, which led to different machines and protocols that are currently available (Olshina \& Sharon, 2016). Generally, two main analysis strategies exist when analyzing proteins through mass spectrometry. The first strategy is called top-down analysis in which proteins of usually known identity are collected as a whole and measured. Top-down strategy is useful to identify posttranslational modifications in proteins of known sequence (Kellie et al., 2010; Olshina \& Sharon, 2016). The second strategy, that happens to be more common, is the bottom-up where all the proteins are purified from cells then digested and run through MS (Kellie et al., 2010; Olshina \& Sharon, 2016).

Posttranslational modification (PTM) is an important regulatory mechanism of proteins. Phosphorylation, methylation, ubiquitination and glycosylation are common forms of modifications among more than 400 other PTMs identified (Minguez et al., 2012). Modification of expressed proteins regulates their activity through adjusting their localization, stability, folding, conformation and function (Saraswathy \& Ramalingam, 2011). Identification and quantification of PTM add another level of information when comparing healthy to malignant cells. Quantifying functionally active proteins is more informative and of higher value than comparing total active and inactive expressed proteins. However, modified proteins with specific modifications are usually many folds less common than their unmodified forms. Therefore, enrichment for peptides with specific modifications is an important step prior to identification and quantification. (Khoury et al., 2011).

Phosphorylation is the most studied posttranslational modification, which regulates most of cellular processes (Nesverova \& Tornroth-Horsefield, 2019). Protein phosphorylation is a reversible process where a phosphate group is added to a side chain of a specific amino acid. In normal conditions, phosphorylation is kept under 
tight regulation through a group of kinases and phosphatases in the cell. With more than 10,000 identified phosphorylation sites, phosphorylation is widespread through the proteome where $30-75 \%$ of the proteome is phosphorylated at any time point (Drake et al., 2016; Ficarro et al., 2002; Saraswathy \& Ramalingam, 2011). Phosphoproteomic analysis using mass spectrometry is a robust method to identify and quantify phosphorylated peptides among the cellular proteome. This robust tool empowers the study of molecular mechanisms of tumorigenesis and allows the discovery of new tumor biomarkers and therapeutic targets like ACTN4 and ARFGEF2 in Burkitt's lymphoma (Corso et al., 2016). Enrichment for phosphopeptides usually depend on phospho-specific antibodies or chromatography techniques like immobilized metal affinity (IMAC) and Titanium Dioxide $\left(\mathrm{TiO}_{2}\right)(\mathrm{Lopez}$ et al., 2012).

\subsubsection{Liquid chromatography-coupled electrospray ionization mass spectrometry (LC-MS/MS)}

In order to analyze any sample on mass spectrometry, samples have to go through five main steps. The first step is sample preparation followed by fractionalization, ionization of peptides, detection and then finally data analysis. Sample preparation includes culturing of target cells; this may include treatment with specific stimulators or inhibitors, which depends on the study. After culturing, cells are lysed with specific lysis buffers in order to extract the protein content. Lysis buffers' properties like $\mathrm{pH}$ values and percentages of detergents and proteases inhibitors are crucial for accurate and high yield experiments (Haupl et al., 2019; Stokes et al., 2012). Lysates are then digested with enzymes to produce peptides of smaller sizes and with specific properties. Trypsin is the most common digestion enzyme, which cuts peptides at the lysine or arginine carboxyl side. Peptides produced from trypsin digestion (tryptic peptides) are very complex and highly concentrated. Accurate acquisition and sensitive measurement of MS need the samples to be fractionized into less condense and less concentrated amounts. Liquid chromatography (LC) offers a convenient and efficient method to fractionize complex peptides into more simple peptides with properties similar to those needed for mass spectrometry (Ligon, 2001). Liquid chromatography coupled online to MS (LC-MS) is a common and robust technique for forming appropriate small peptide fractions and enables 
measurements at femtomole level (Figure 4) (Haupl et al., 2019; Ligon, 2001; Stokes et al., 2012).

To enable identification and quantification, peptides have to be ionized into charged ions. Peptides are transformed into detectable ions through one of different ionization techniques. Electronspray ionization (ESI) and matrix-assisted laser desorption (MALDI) are the two most common ionization techniques. In ESI, peptides dissolved in solution are sprayed under high voltage and negative pressure forming tiny charged droplets or ions. Ions on this level usually are multiply charged and are detected by an analyzer as mas over charge or Thomson value $(\mathrm{m} / \mathrm{z})$ (Ho et al., 2003). Specific ions are chosen as precursors for further fragmentation into singly charged ions, which are then detected (second MS or MS/MS). Analysis of MS data depends on comparing masses of measured peptides to databases. These databases contain all theoretical peptide masses resulted from virtual tryptic digestion of the whole proteome (Haupl et al., 2019; Ho et al., 2003; Stokes et al., 2012).

\subsection{Stable Isotope Labeling with Amino acids (SILAC) labeling}

Mass spectrometry is not inherently a quantitative method but rather a qualitative method that is able to detect peptides and hence identify proteins. Therefore, MS analysis is usually coupled to other tools that can make it a quantitative tool. Quantification using MS is achievable through comparing quantities of peptides between two samples. Those samples can be labelled or label free and can be used for relative or absolute quantification (Ankney et al., 2018). Sample labeling can take metabolic or chemical form. Stable Isotope Labeling with Amino acids (SILAC) is considered a common and straightforward labeling method, which enables relative quantification of different samples within the same MS analysis (Ankney et al., 2018). The method depends on feeding cells with special culture medium containing heavy amino acids instead of normal amino acids. Following multiple cycles of culture, cells incorporate heavy amino acids in their proteins and become distinguishable from their normal light counterparts. Heavily labelled proteins are mixed with light/normal proteins and measured together through MS (X. Wang et al., 2018). Arginine 6/10 and Lysine 4/8 are examples of heavy amino acids that are commonly used (Figure 4) (X. L. Chen et al., 2015; X. Wang et al., 2018). 
Introduction

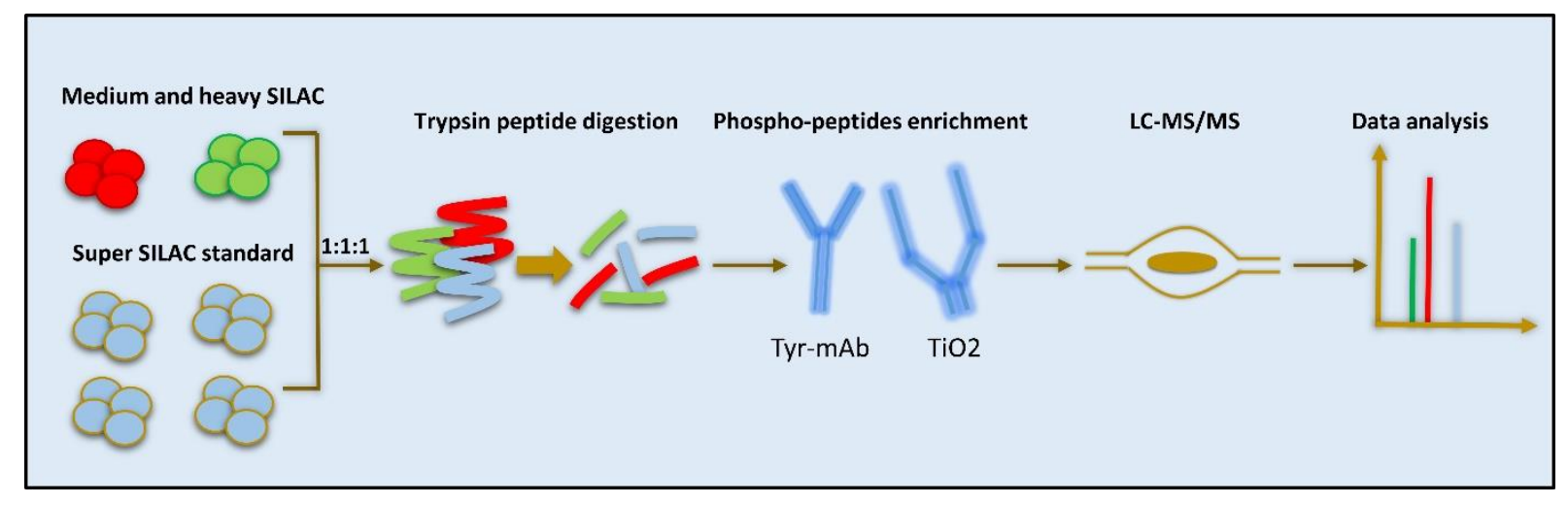

Figure 4 Summary of LC-MS/MS phosphoproteomic analysis sample preparation. 
Introduction

\subsection{Aim of the Study}

For decades lung cancer is the leading type of cancer in terms of incident rates and mortality. Molecular therapy is considered a promising and effective way to target lung cancer cells. Unlike adenocarcinoma of the lung, squamous cell lung cancer and small cell lung cancer subgroups lack any approved molecular targets. Fibroblast growth factor receptor 1 (FGFR1) is considered an encouraging new target due to its high prevalence of amplification in SQCLC and SCLC patients and efficacy in clinical trials. However, a high proportion of lung cancer patients with FGFR1 amplification have shown initial resistance or acquired resistance to FGFR1 targeted therapy.

The aim of the current thesis is to improve selection criteria for patients who will benefit from FGFR1 targeted therapy and to investigate and confront mechanisms of resistance to FGFR1 inhibition in lung cancer cells. The key objectives are:

a. Screening prevalence of FGFR1 gene amplification and protein expression in lung cancer patient tissue samples using fluorescence in situ hybridization (FISH) and immunohistochemistry (IHC), respectively.

b. Examining correlation between FGFR1 gene amplification and protein expression in lung cancer patient tissue samples to help improving selection criteria for patients who benefit from FGFR1 targeted therapy.

c. Investigating intrinsic and induced mechanisms and signaling pathways of resistance to FGFR1 inhibition in lung cancer cells using phosphoproteomic mass spectrometric analysis.

d. Characterizing and functional validating new molecular targets, which can sensitize lung cancer resistant cells to FGFR1 inhibition therapy. 


\section{Materials and Methods}

\subsection{Materials}

\subsubsection{Equipment}

\begin{tabular}{|c|c|}
\hline Equipment & Suppliers \\
\hline Autoclave & Systec VX 100 \\
\hline Autostainerlink48 & $\begin{array}{l}\text { Agilent technologies, California, } \\
\text { USA }\end{array}$ \\
\hline $\begin{array}{l}\text { Bacterial incubator, Heraeus instruments } \\
\text { function line }\end{array}$ & $\begin{array}{l}\text { Thermo Fischer Scientific, } \\
\text { Waltham, USA }\end{array}$ \\
\hline Bacterial shaker, orbital shaker & VWR, Pennsylvania, USA \\
\hline Eppendorf Centrifuge 5424 & Eppendorf, Hamburg, Germany \\
\hline Eppendorf Centrifuge 5430R & Eppendorf, Hamburg, Germany \\
\hline FACSAria sorter & BD, California, USA \\
\hline Fusion Fx, peQlab & VILBER \\
\hline Genetic analyzser 3500 & $\begin{array}{l}\text { Applied Biosystems, } \\
\text { Massachusetts, USA }\end{array}$ \\
\hline $\begin{array}{l}\text { Herasafe, biological culture hood, Heraeus } \\
\text { instruments }\end{array}$ & $\begin{array}{l}\text { Thermo Fischer Scientific, } \\
\text { Waltham, USA }\end{array}$ \\
\hline MiSeq® System, SY-410-1003 & Illumnia Inc., USA \\
\hline Mr. Frosty ${ }^{\mathrm{TM}}$ Gefrierbehälter & $\begin{array}{l}\text { Thermo Fischer Scientific, } \\
\text { Waltham, USA }\end{array}$ \\
\hline Muse Guava cell analyzer & Luminex \\
\hline Nanodrop 2000/c & $\begin{array}{l}\text { Thermo Fischer Scientific, } \\
\text { Waltham, USA }\end{array}$ \\
\hline Plate reader, TECAN 200M pro & $\begin{array}{l}\text { TECAN, maennedorf, } \\
\text { Switzerland }\end{array}$ \\
\hline Primovert Microscope & $\begin{array}{l}\text { CARL ZEISS, Oberochen, } \\
\text { Germany }\end{array}$ \\
\hline Privileg $8018 \mathrm{e}$ microwave & Privileg, Stuttgart, Germany \\
\hline $\begin{array}{l}\text { Q Exactive }{ }^{\mathrm{TM}} \mathrm{HF} \text { Hybrid Quadrupol- } \\
\text { Orbitrap }^{\mathrm{TM}} \text { Massenspektrometer }\end{array}$ & $\begin{array}{l}\text { Thermo Fischer Scientific, } \\
\text { Waltham, USA }\end{array}$ \\
\hline QIAxcel & QIAGEN, Venlo, Netherlands \\
\hline Sonicator, Bandelin Sonopuls HD70 & Bandelin, Berlin, Germany \\
\hline
\end{tabular}




\begin{tabular}{|l|l|}
\hline Speed Vacuum concentrator & $\begin{array}{l}\text { Thermo Fischer Scientific, } \\
\text { Waltham, USA }\end{array}$ \\
\hline Thermocycler, peqSTAR & Peqlab \\
\hline Trans-Blot Turbo & Bio-Rad, Hercules, USA \\
\hline Tubes vortex, schuett labortechnik & LABO \\
\hline UltiMate $^{\text {TM }} 3000$ RSLCnano System & $\begin{array}{l}\text { Thermo Fischer Scientific, } \\
\text { Waltham, USA }\end{array}$ \\
\hline VP2000 processor system & $\begin{array}{l}\text { Abbott Molecular, Wiesbaden, } \\
\text { Germany }\end{array}$ \\
\hline WTC binder Cell lines incubator, & Binder \\
\hline ZOE fluorescent cell imager & Bio-Rad, Hercules, USA \\
\hline
\end{tabular}

\subsubsection{Antibodies}

\begin{tabular}{|l|l|l|l|}
\hline Antibody & Technique & Dilution & Supplier \\
\hline Akt \#9272 & WB & $1: 10000$ & $\begin{array}{l}\text { Cell Signaling Technology } \\
\text { Europe, Frankfurt am } \\
\text { Main, Germany }\end{array}$ \\
\hline $\begin{array}{l}\text { ERK, (Erk1/2) Antibody } \\
\# 9102\end{array}$ & WB & $1: 10000$ & $\begin{array}{l}\text { Cell Signaling Technology } \\
\text { Europe, Frankfurt am } \\
\text { Main, Germany }\end{array}$ \\
\hline FGFR1 (D8E4) \#9740 & WB, IHC & $1: 10000$ & $\begin{array}{l}\text { Cell Signaling Technology } \\
\text { Europe, Frankfurt am } \\
\text { Main, Germany }\end{array}$ \\
\hline $\begin{array}{l}\text { FGFR1 antibody } \\
\text { (ab10646) }\end{array}$ & WB, IHC & $1: 5000$ & \begin{tabular}{l} 
Abcam, Germany \\
\hline MEK1/2 Antibody \#9122
\end{tabular} \\
WB & $1: 10000$ & $\begin{array}{l}\text { Cell Signaling Technology } \\
\text { Europe, Frankfurt am } \\
\text { Main, Germany }\end{array}$ \\
\hline $\begin{array}{l}\text { PARK7 (ab18257) } \\
\text { Phospho-Akt (Ser473) }\end{array}$ & WB, IHC & $1: 10000$ & $\begin{array}{l}\text { Cell Signaling Technology } \\
\text { Europe, Frankfurt am } \\
\text { Main, Germany }\end{array}$ \\
\hline
\end{tabular}




\begin{tabular}{|c|c|c|c|}
\hline $\begin{array}{l}\text { Phospho-Akt (Thr308) } \\
\# 9275\end{array}$ & WB & $1: 10000$ & $\begin{array}{l}\text { Cell Signaling Technology } \\
\text { Europe, Frankfurt am } \\
\text { Main, Germany }\end{array}$ \\
\hline $\begin{array}{l}\text { Phospho-Akt1 (S473) } \\
\text { antibody [EP2109Y] } \\
\text { \#ab81283 }\end{array}$ & $\mathrm{IHC}$ & $1: 100$ & Abcam, Germany \\
\hline Phospho-CD44 (pSer706) & WB & $1: 10000$ & $\begin{array}{l}\text { Sigma-Aldrich Co., St. } \\
\text { Louis, USA }\end{array}$ \\
\hline $\begin{array}{l}\text { Phospho-ERK(C33E10) } \\
\text { Rabbit mAb \#3192 }\end{array}$ & WB & $1: 10000$ & $\begin{array}{l}\text { Cell Signaling Technology } \\
\text { Europe, Frankfurt am } \\
\text { Main, Germany }\end{array}$ \\
\hline $\begin{array}{l}\text { Phospho-FAK } \\
\text { (Tyr576/577) \#3281T }\end{array}$ & WB & $1: 10000$ & $\begin{array}{l}\text { Cell Signaling Technology } \\
\text { Europe, Frankfurt am } \\
\text { Main, Germany }\end{array}$ \\
\hline $\begin{array}{l}\text { Phospho-FGFR1 } \\
\text { (Tyr653/654) (D4X3D) }\end{array}$ & WB & $1: 10000$ & $\begin{array}{l}\text { Cell Signaling Technology } \\
\text { Europe, Frankfurt am } \\
\text { Main, Germany }\end{array}$ \\
\hline $\begin{array}{l}\text { Phospho-MEK1/2 } \\
\text { (Ser217/221) \#9121 }\end{array}$ & WB & $1: 10000$ & $\begin{array}{l}\text { Cell Signaling Technology } \\
\text { Europe, Frankfurt am } \\
\text { Main, Germany }\end{array}$ \\
\hline $\begin{array}{l}\text { Phospho-PAK1 } \\
\text { (Thr423)/PAK2 (Thr402) } \\
\text { \#2601 }\end{array}$ & WB & $1: 10000$ & $\begin{array}{l}\text { Cell Signaling Technology } \\
\text { Europe, Frankfurt am } \\
\text { Main, Germany }\end{array}$ \\
\hline $\begin{array}{l}\text { Phospho-PI3 Kinase p85 } \\
\text { (Tyr458)/p55 (Tyr199) } \\
\# 4228\end{array}$ & WB & $1: 10000$ & $\begin{array}{l}\text { Cell Signaling Technology } \\
\text { Europe, Frankfurt am } \\
\text { Main, Germany }\end{array}$ \\
\hline $\begin{array}{l}\text { Phospho-Stat3 (Tyr705) } \\
\# 9131\end{array}$ & WB & $1: 10000$ & $\begin{array}{l}\text { Cell Signaling Technology } \\
\text { Europe, Frankfurt am } \\
\text { Main, Germany }\end{array}$ \\
\hline $\begin{array}{l}\text { Polyclonal Goat anti- } \\
\text { mouse, HRP }\end{array}$ & WB & $1: 10000$ & Agilent, California, USA \\
\hline $\begin{array}{l}\text { Polyclonal Goat anti-rabbit, } \\
\text { HRP }\end{array}$ & WB & $1: 10000$ & Agilent, California, USA \\
\hline
\end{tabular}




\begin{tabular}{|l|l|l|l|}
\hline Propidium lodide stain & FACS & $\begin{array}{l}10 \\
\mu \mathrm{g} / \mathrm{mL}\end{array}$ & $\begin{array}{l}\text { Thermo Fischer Scientific, } \\
\text { Waltham, USA }\end{array}$ \\
\hline $\begin{array}{l}\text { PTEN (138G6) Rabbit } \\
\text { mAb \#9559 }\end{array}$ & WB & $1: 10000$ & $\begin{array}{l}\text { Cell Signaling Technology } \\
\text { Europe, Frankfurt am } \\
\text { Main, Germany }\end{array}$ \\
\hline $\begin{array}{l}\text { PTMScan® Phospho- } \\
\text { Tyrosine Rabbit mAb (P- } \\
\text { Tyr-1000) Kit \#8803 }\end{array}$ & $\begin{array}{l}\text { Tyrosine } \\
\text { peptides } \\
\text { enrichment }\end{array}$ & & $\begin{array}{l}\text { Cell Signaling Technology } \\
\text { Europe, Frankfurt am } \\
\text { Main, Germany }\end{array}$ \\
\hline $\begin{array}{l}\text { ZytoLight SPEC } \\
\text { FGFR1/CEN 8 }\end{array}$ & FISH & & $\begin{array}{l}\text { ZytoVision, Bremerhaven, } \\
\text { Germany }\end{array}$ \\
\hline
\end{tabular}

\subsubsection{Cell lines}

\begin{tabular}{|c|c|c|}
\hline Cell line & Culture medium & Supplier \\
\hline $\begin{array}{l}\text { DMS114, CRL-2066, } \\
\text { human SCLC }\end{array}$ & $\begin{array}{l}\text { RPMI } 1640,10 \% \text { FCS, } 1 \% \\
\text { Glutamine and } 1 \% \text { penecillin- } \\
\text { streptomycin }\end{array}$ & $\begin{array}{l}\text { National } \\
\text { Institutes of } \\
\text { Health, National } \\
\text { Cancer Institute, } \\
\text { Frederick, MD, } \\
\text { United States }\end{array}$ \\
\hline DMS-53, human SCLC & $\begin{array}{l}\text { Waymouth's MB 752/1 Medium, } \\
\text { 10\% FCS, 1\% Glutamine and 1\% } \\
\text { penecillin-streptomycin }\end{array}$ & $\begin{array}{l}\text { AddexBio } \\
\text { Technologies, } \\
\text { USA }\end{array}$ \\
\hline EBC-1, human SQCLC & $\begin{array}{l}\text { RPMI 1640, 10\% FCS, } 1 \% \\
\text { Glutamine and 1\% penecillin- } \\
\text { streptomycin }\end{array}$ & $\begin{array}{l}\text { JCRB Cell Bank } \\
\text { (Japanese } \\
\text { Collection of } \\
\text { Research } \\
\text { Bioresources } \\
\text { Cell Bank) }\end{array}$ \\
\hline H1339, human SCLC & $\begin{array}{l}\text { RPMI } 1640,10 \% \text { FCS, } 1 \% \\
\text { Glutamine and } 1 \% \text { penecillin- } \\
\text { streptomycin }\end{array}$ & ATCC, Wesel \\
\hline $\begin{array}{l}\text { H1581, human large cell } \\
\text { LC }\end{array}$ & $\begin{array}{l}\text { RPMI 1640/DMEM/F12 1:1, 10\% } \\
\text { FCS, } 1 \% \text { Glutamine and 1\% }\end{array}$ & $\begin{array}{l}\text { AddexBio } \\
\text { Technologies, }\end{array}$ \\
\hline
\end{tabular}




\begin{tabular}{|c|c|c|}
\hline & penecillin-streptomycin & USA \\
\hline H1703, human SQCLC & $\begin{array}{l}\text { RPMI } 1640,10 \% \text { FCS, } 1 \% \\
\text { Glutamine and } 1 \% \text { penecillin- } \\
\text { streptomycin }\end{array}$ & $\begin{array}{l}\text { AddexBio } \\
\text { Technologies, } \\
\text { USA }\end{array}$ \\
\hline $\begin{array}{l}\text { H2170, CRL-5928, } \\
\text { human SQCLC }\end{array}$ & $\begin{array}{l}\text { RPMI } 1640,10 \% \text { FCS, } 1 \% \\
\text { Glutamine and } 1 \% \text { penecillin- } \\
\text { streptomycin }\end{array}$ & ATCC, Wesel \\
\hline H226, human SQCLC & $\begin{array}{l}\text { RPMI 1640, 10\% FCS, } 1 \% \\
\text { Glutamine and } 1 \% \text { penecillin- } \\
\text { streptomycin }\end{array}$ & $\begin{array}{l}\text { AddexBio } \\
\text { Technologies, } \\
\text { USA }\end{array}$ \\
\hline $\begin{array}{l}\text { H-520, HTB-182, human } \\
\text { SQCLC }\end{array}$ & $\begin{array}{l}\text { RPMI } 1640,10 \% \text { FCS, } 1 \% \\
\text { Glutamine and } 1 \% \text { penecillin- } \\
\text { streptomycin }\end{array}$ & ATCC, Wesel \\
\hline H69, human SCLC & $\begin{array}{l}\text { RPMI } 1640,10 \% \text { FCS, } 1 \% \\
\text { Glutamine and } 1 \% \text { penecillin- } \\
\text { streptomycin }\end{array}$ & ATCC, Wesel \\
\hline $\begin{array}{l}\text { H82, HTB-175, human } \\
\text { SCLC }\end{array}$ & $\begin{array}{l}\text { RPMI 1640, 10\% FCS, } 1 \% \\
\text { Glutamine and } 1 \% \text { penecillin- } \\
\text { streptomycin }\end{array}$ & ATCC, Wesel \\
\hline $\begin{array}{l}\text { HCC-15, ACC 496, } \\
\text { human SQCLC }\end{array}$ & $\begin{array}{l}\text { RPMI 1640, 10\% FCS, } 1 \% \\
\text { Glutamine and 1\% penecillin- } \\
\text { streptomycin }\end{array}$ & $\begin{array}{l}\text { DSMZ-German } \\
\text { Collection of } \\
\text { Microorganisms } \\
\text { and Cell } \\
\text { Cultures GmbH }\end{array}$ \\
\hline HCC33, human SCLC & $\begin{array}{l}\text { RPMI } 1640,10 \% \text { FCS, } 1 \% \\
\text { Glutamine and } 1 \% \text { penecillin- } \\
\text { streptomycin }\end{array}$ & $\begin{array}{l}\text { DSMZ-German } \\
\text { Collection of } \\
\text { Microorganisms } \\
\text { and Cell } \\
\text { Cultures GmbH }\end{array}$ \\
\hline $\begin{array}{l}\text { LCLC103H, human large } \\
\text { cell LC }\end{array}$ & $\begin{array}{l}\text { RPMI 1640, 10\% FCS, } 1 \% \\
\text { Glutamine and } 1 \% \text { penecillin- } \\
\text { streptomycin }\end{array}$ & $\begin{array}{l}\text { DSMZ-German } \\
\text { Collection of } \\
\text { Microorganisms } \\
\text { and Cell }\end{array}$ \\
\hline
\end{tabular}




\begin{tabular}{|c|c|c|}
\hline & & Cultures $\mathrm{GmbH}$ \\
\hline LK-2, human SQCLC & $\begin{array}{l}\text { RPMI } 1640,10 \% \text { FCS, } 1 \% \\
\text { Glutamine and } 1 \% \text { penecillin- } \\
\text { streptomycin }\end{array}$ & $\begin{array}{l}\text { JCRB Cell Bank } \\
\text { (Japanese } \\
\text { Collection of } \\
\text { Research } \\
\text { Bioresources } \\
\text { Cell Bank) }\end{array}$ \\
\hline $\begin{array}{l}\mathrm{OH} 1, \mathrm{CVCL} \_\mathrm{A} 765 \\
\text { human SCLC }\end{array}$ & $\begin{array}{l}\text { RPMI 1640, 10\% FCS, } 1 \% \\
\text { Glutamine and } 1 \% \text { penecillin- } \\
\text { streptomycin }\end{array}$ & ATCC, Wesel \\
\hline $\begin{array}{l}\text { OH3, CVCL_A766, } \\
\text { human SCLC }\end{array}$ & $\begin{array}{l}\text { RPMI 1640, 10\% FCS, } 1 \% \\
\text { Glutamine and 1\% penecillin- } \\
\text { streptomycin }\end{array}$ & ATCC, Wesel \\
\hline SCLC21H, human SCLC & $\begin{array}{l}\text { RPMI 1640, 10\% FCS, } 1 \% \\
\text { Glutamine and 1\% penecillin- } \\
\text { streptomycin }\end{array}$ & ATCC, Wesel \\
\hline SHP77, human SCLC & $\begin{array}{l}\text { RPMI } 1640,10 \% \text { FCS, } 1 \& \\
\text { Glutamine and } 1 \% \text { penecillin- } \\
\text { streptomycin }\end{array}$ & ATCC, Wesel \\
\hline $\begin{array}{l}\text { SK-MES-1, human } \\
\text { SQCLC }\end{array}$ & $\begin{array}{l}\text { EMEM (C0005-01), 10\% FCS, 1\% } \\
\text { Glutamine and 1\% penecillin- } \\
\text { streptomycin }\end{array}$ & $\begin{array}{l}\text { AddexBio } \\
\text { Technologies, } \\
\text { USA }\end{array}$ \\
\hline SW2, human SCLC & $\begin{array}{l}\text { RPMI 1640, 10\% FCS, } 1 \% \\
\text { Glutamine and } 1 \% \text { penecillin- } \\
\text { streptomycin }\end{array}$ & ATCC, Wesel \\
\hline
\end{tabular}

\subsection{4. siRNAs and guide RNAs}

\begin{tabular}{|l|l|}
\hline siRNA/gRNA & Supplier \\
\hline $\begin{array}{l}\text { AllStars Neg. Control siRNA (20 nmol) Cat } \\
\text { No./ID: } 1027281\end{array}$ & Qiagen GmbH, Hilden \\
\hline $\begin{array}{l}\text { Crispr, fwd, primer: } \\
\text { GGGGCTCTTACCCCAATGTT }\end{array}$ & Eurofins, Ebersberg, Germany \\
\hline Crispr, rev, primer: & Eurofins, Ebersberg, Germany \\
\hline
\end{tabular}




\begin{tabular}{|l|l|}
\hline CCTGAGGCTACGCAGAAAGT & \\
\hline $\begin{array}{l}\text { gRNApast1fwd 5'-P- } \\
\text { CACCGGGAGCACCAGTGTAGCCAGG-3' }\end{array}$ & Eurofins, Ebersberg, Germany \\
\hline $\begin{array}{l}\text { gRNApast1rev5'- } \\
\text { AAACCCTGGCTACACTGGTGCTCCC-3' }\end{array}$ & Eurofins, Ebersberg, Germany \\
\hline $\begin{array}{l}\text { gRNApre1fwd 5'-P- } \\
\text { CACCGTTCCCAGGTCCCCTAAGAGG-3' }\end{array}$ & Eurofins, Ebersberg, Germany \\
\hline $\begin{array}{l}\text { gRNApre1rev 5'- } \\
\text { AAACCCTCTTAGGGGACCTGGGAAC-3' }\end{array}$ & Eurofins, Ebersberg, Germany \\
\hline Hs_FGFR1_6 FlexiTube siRNA & Qiagen GmbH, Hilden \\
\hline Hs_FGFR1_7 FlexiTube siRNA & Qiagen GmbH, Hilden \\
\hline $\begin{array}{l}\text { U6 primer, } \\
\text { 5'GAGGGCCTATTTCCCATGATTCC-3' }\end{array}$ & Eurofins, Ebersberg, Germany \\
\hline Hs_CD44_5 FlexiTube siRNA & Qiagen GmbH, Hilden \\
\hline
\end{tabular}

\subsubsection{Plasmids}

\begin{tabular}{|l|l|}
\hline Plasmids & Suppliers \\
\hline 1036 pcDNA3 Myr HA Akt1 & (Plasmid \#9008), Addgene \\
\hline 1436 pcDNA3 Flag HA & (Plasmid \#10792), Addgene \\
\hline pSpCas9(BB)-2A-GFP (PX458) & (Plasmid \#48138), Addgene \\
\hline pU6-(Bbsl)_CBh-Cas9-T2A-mCherry & (Plasmid \#64324), Addgene \\
\hline
\end{tabular}

\subsubsection{Buffers and media}

Unless otherwise stated, buffers and media were dissolved in distilled water $\left(\mathrm{ddH}_{2} \mathrm{O}\right)$. LB growth media were autoclaved at $125{ }^{\circ} \mathrm{C}$ then stored at $4{ }^{\circ} \mathrm{C}$. Culture media serums were heat deactivated at $56{ }^{\circ} \mathrm{C}$ then stored at $-20{ }^{\circ} \mathrm{C}$. Glutamine and penicillin-streptomycin aliquots were stored at $-20^{\circ} \mathrm{C}$. Buffers and media were stored at room temperature, unless otherwise stated.

\begin{tabular}{|l|l|}
\hline Reagent & Composition \\
\hline $70 \%$ Ethanol & $700 \mathrm{~mL}$ Ethanol (99.9\%) in $1 \mathrm{~L} \mathrm{ddH} 2 \mathrm{O}$ \\
\hline Blocking buffer & $5 \%$ milk powder in TBS-T \\
\hline
\end{tabular}




\begin{tabular}{|c|c|}
\hline DMEM & $10 \%$ FCS, $1 \%$ Penstrip \& $1 \%$ glutamine \\
\hline Freezing medium & $5 \%$ DMSO in FCS \\
\hline Global proteome lysis buffer & $\begin{array}{l}\text { 50mM Tris-HCL PH 7,5, 150mM NACL, } 1 \mathrm{mM} \\
\text { EDTA-disodium salt, } 1 \%(\mathrm{v} / \mathrm{v}) \mathrm{NP}-40,0.1 \% \\
\text { (w/v) Na-deoxycholate, } 1 \text { tab Roche complete } \\
\text { protease inhibitor cocktail, } 5 \mathrm{mM} \text { B- } \\
\text { glycerophosphate, } 5 \mathrm{mM} \mathrm{NaF,} 1 \mathrm{mM} \mathrm{Na-} \\
\text { orthovandate, } 10 \mathrm{mM} \mathrm{N} \text {-ethylmaleimide (NEM) } \\
\text { and LC-MS grade water }\end{array}$ \\
\hline Heavy SILAC medium & $\begin{array}{l}\text { SILAC medium, 50mL dialyzed FCS, } 5 \mathrm{~mL} \\
\text { glutamine, } 5 \mathrm{~mL} \text { Penstrip, 5,5mL Na-pyruvate } \\
\text { solution, } 5,5 \mathrm{~mL} \text { ArgH \& 5,5mL LysH }\end{array}$ \\
\hline LB- Agar & $15 \mathrm{~g} / \mathrm{L}$ Agar-Agar in LB medium \\
\hline LB medium & 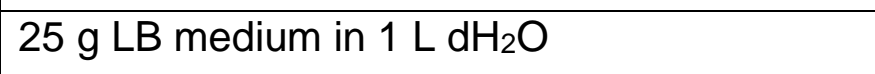 \\
\hline Loading buffer $(1: 1 / 3)$ & $\begin{array}{l}500 / 250 \mu \mathrm{L} 2 \times / 4 x \text { Laemmli Sample Buffer, } \\
500 / 750 \mu \mathrm{L} \text { 2-Mercaptoethanol }\end{array}$ \\
\hline Medium SILAC medium & $\begin{array}{l}\text { SILAC medium, 50mL dialyzed FCS, } 5 \mathrm{~mL} \\
\text { glutamine, } 5 \mathrm{~mL} \text { Penstrip, 5,5mL Na-pyruvate } \\
\text { solution, 5,5mL ArgM \& 5,5mL LysM }\end{array}$ \\
\hline NP40 Lysis buffer & $\begin{array}{l}50 \mathrm{mM} \text { Tris/HCl }(\mathrm{pH} 7.6), 150 \mathrm{mM} \mathrm{NaCl}, 5 \mathrm{mM} \\
\mathrm{NaF}, 1 \% \mathrm{NP}-40\end{array}$ \\
\hline PBS & $9.55 \mathrm{~g} / \mathrm{L} \mathrm{PBS}$ Dulbecco in $\mathrm{ddH}_{2} \mathrm{O}$ \\
\hline Ponceau & $\begin{array}{l}0.5 \mathrm{~g} \text { Ponceau, } 5 \mathrm{~mL} 100 \% \text { glacial acetic acid } \\
\text { in } 500 \mathrm{~mL} \text { with } \mathrm{ddH}_{2} \mathrm{O}\end{array}$ \\
\hline pYome lysis buffer & $\begin{array}{l}\text { 20mM HEPES PH8, 2.5mM Sodium } \\
\text { pyrophosphate tetrabasic decahydrate, } 1 \mathrm{mM} \\
\beta \text {-Glycerophosphate disodium salt hydrate, } \\
1 \mathrm{mM} \text { Naorthovandate NaVO4, } 8 \mathrm{M} \text { Urea, LC- } \\
\text { MS grade water }\end{array}$ \\
\hline RPMI-1640 & $10 \%$ FCS, $1 \%$ Penstrip \& $1 \%$ glutamine \\
\hline TBE buffer & $100 \mathrm{~mL} 10 \times$ TBE buffer in $1 \mathrm{~L} \mathrm{ddH}_{2} \mathrm{O}$ \\
\hline TBS & $\begin{array}{l}4.24 \mathrm{~g} \text { Tris, } 292.7 \mathrm{~g} \mathrm{NaCl}, 26 \mathrm{~g} \text { Tris- } \mathrm{HCl} \\
\text { solved in } 1 \mathrm{~L} \mathrm{ddH}_{2} \mathrm{O}\end{array}$ \\
\hline
\end{tabular}




\begin{tabular}{|c|c|}
\hline TBS-T & 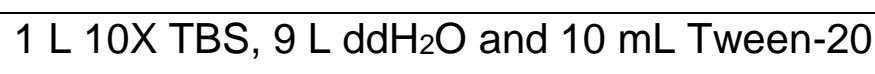 \\
\hline Waymouth's MB 752/1 & $10 \%$ FCS, $1 \%$ Penstrip \& $1 \%$ glutamine \\
\hline Westernblotting running buffer & $\begin{array}{l}100 \mathrm{~mL} \text { 10X Tris/Glycine/SDS Buffer in } 1 \mathrm{~L} \\
\mathrm{ddH}_{2} \mathrm{O}\end{array}$ \\
\hline
\end{tabular}

\subsubsection{Reagents and kits}

\begin{tabular}{|c|c|}
\hline Reagent & Supplier \\
\hline 10X Tango Buffer & $\begin{array}{l}\text { Thermo Fischer Scientific, Waltham, } \\
\text { USA }\end{array}$ \\
\hline 10X Tango buffer & $\begin{array}{l}\text { Thermo Fischer Scientific, Waltham, } \\
\text { USA }\end{array}$ \\
\hline 10X Tris-Glycine-SDS Buffer & Bio-Rad, Feldkirchen, Germany \\
\hline 2x Laemmli Sample Buffer & Bio-Rad, Feldkirchen, Germany \\
\hline 4x Laemmli Sample Buffer & Bio-Rad, Feldkirchen, Germany \\
\hline 6X loading Dye & $\begin{array}{l}\text { Thermo Fischer Scientific, Waltham, } \\
\text { USA }\end{array}$ \\
\hline Agar-Agar & Carl Roth GmbH \& Co. KG, Karlsruhe \\
\hline Ambion $\AA$ DEPC-treated water & Invitrogen, Massachusetts, USA \\
\hline Ampicillin & AppliChem GmbH, Darmstadt \\
\hline Ampicillin & Gibco, Massachusetts, USA \\
\hline Archer ${ }^{\circledR}$ FusionPlex® CTL Panel & Illumnia, San Diego, California, USA \\
\hline ATP $(10 \mathrm{mM})$ & $\begin{array}{l}\text { Thermo Fischer Scientific, Waltham, } \\
\text { USA }\end{array}$ \\
\hline BigDye XTerminator ${ }^{\mathrm{TM}}$ Purification Kit & $\begin{array}{l}\text { Applied Biosystems, Massachusetts, } \\
\text { USA }\end{array}$ \\
\hline Buffer $\mathrm{O}$ & $\begin{array}{l}\text { Thermo Fischer Scientific, Waltham, } \\
\text { USA }\end{array}$ \\
\hline Celltiter 96 Aqueous one solution & $\begin{array}{l}\text { Thermo Fischer Scientific, Waltham, } \\
\text { USA }\end{array}$ \\
\hline DAB Substrate Kit & Agilent Technologies, California, USA \\
\hline $\mathrm{DC}^{\mathrm{TM}}$ Protein Assay & Bio-Rad, Feldkirchen, Germany \\
\hline Dh5alpha competent E. coli & $\begin{array}{l}\text { Thermo Fischer Scientific, Waltham, } \\
\text { USA }\end{array}$ \\
\hline
\end{tabular}




\begin{tabular}{|l|l|}
\hline $\begin{array}{l}\text { Dialyzed fetal bovine serum (dialyzed } \\
\text { FbS) }\end{array}$ & Gibco, Massachusetts, USA \\
\hline DMEM medium & Gibco, Massachusetts, USA \\
\hline dNTPs & $\begin{array}{l}\text { New England Biolabs GmbH, Frankfurt } \\
\text { am Main, Germany }\end{array}$ \\
\hline DTT (10mM) & $\begin{array}{l}\text { Thermo Fischer Scientific, Waltham, } \\
\text { USA }\end{array}$ \\
\hline EDTA & Merck, Darmstadt, Germany \\
\hline pnVision Flex Target Retrieval Solution, & Agilent Technologies, California, USA \\
\hline $\begin{array}{l}\text { ExoSAP-IT Express PCR Cleanup } \\
\text { Reagents }\end{array}$ & $\begin{array}{l}\text { Thermo Fischer Scientific, Waltham, } \\
\text { USA }\end{array}$ \\
\hline Fast Digest BbsI (Bpil) & $\begin{array}{l}\text { New England Biolabs GmbH, Frankfurt } \\
\text { am Main, Germany }\end{array}$ \\
\hline FastDigest Bbsl & $\begin{array}{l}\text { Thermo Fischer Scientific, Waltham, } \\
\text { USA }\end{array}$ \\
\hline FastDigest Buffer (10X) & $\begin{array}{l}\text { Thermo Fischer Scientific, Waltham, } \\
\text { USA }\end{array}$ \\
\hline FastDigest Green Buffer (10X) & $\begin{array}{l}\text { Thermo Fischer Scientific, Waltham, } \\
\text { USA }\end{array}$ \\
\hline Fetal bovine serum (FbS), quantified Hi & Gibco, Massachusetts, USA \\
\hline Glutamine & Gibco, Massachusetts, USA \\
\hline Hipercfect & QIAGEN, Venlo, Netherlands \\
\hline HistoGel ${ }^{\text {TM }}$ & $\begin{array}{l}\text { Thermo Fischer Scientific, Waltham, } \\
\text { USA }\end{array}$ \\
\hline Lauryl-B-D-maltoside & Merck, Darmstadt, Germany \\
\hline LB medium & Carl Roth GmbH \& Co. KG, Karlsruhe \\
\hline Lipofectamine 3000 & Invitrogen, Massachusetts, USA \\
\hline L-LYSINE:2HCL (13C6, 99\%; 15N2, & $\begin{array}{l}\text { Cambridge Isotope Laboratories, Inc., } \\
\text { USA }\end{array}$ \\
\hline Milk powder & Carl Roth GmbH \& Co. KG, Karlsruhe \\
\hline Mini-PROTEAN, Precast Gels & Bio-Rad, Feldkirchen, Germany \\
\hline Na-pyruvate solution, 11360070 $($ Lys H) & \\
\hline
\end{tabular}




\begin{tabular}{|c|c|}
\hline $\begin{array}{l}\text { Nitrocellulose membranes (Trans-Blot } \\
\text { Bio-Rad Laboratories }\end{array}$ & Bio-Rad, Hercules, USA \\
\hline P3000 reagent & Invitrogen, Massachusetts, USA \\
\hline PageRuler, Prestained 10 to $250 \mathrm{kDa}$ & $\begin{array}{l}\text { Thermo Fischer Scientific, Waltham, } \\
\text { USA }\end{array}$ \\
\hline Penicillin/Streptomycin $(10,000 \mathrm{U} / \mathrm{mL})$ & Gibco, Massachusetts, USA \\
\hline Penicillin/Streptomycin, 15140163 & Gibco, Massachusetts, USA \\
\hline $\begin{array}{l}\text { Phosphate buffered saline (PBS) } \\
\text { Dulbecco }\end{array}$ & Merck, Darmstadt, Germany \\
\hline Pierce $^{\mathrm{TM}} \mathrm{BCA}$ Protein Assay Kit & $\begin{array}{l}\text { Thermo Fischer Scientific, Waltham, } \\
\text { USA }\end{array}$ \\
\hline Purgene $\AA^{\circledR}$ Core Kit $A$ & QIAGEN, Venlo, Netherlands \\
\hline QIAquick PCR Purification Kit & QIAGEN, Venlo, Netherlands \\
\hline $\begin{array}{l}\text { Reblot Plus Strong antibody stripping } \\
\text { solution }\end{array}$ & Merck, Darmstadt, Germany \\
\hline Rnase inhibitor20 $\mathrm{U} / \mu \mathrm{L}$ & $\begin{array}{l}\text { Thermo Fischer Scientific, Waltham, } \\
\text { USA }\end{array}$ \\
\hline RPMI-1640 medium & Gibco, Massachusetts, USA \\
\hline SILAC RPMI 1640 medium & Gibco, Massachusetts, USA \\
\hline $\begin{array}{l}\text { StarPure AGAROSE Low EEO } \\
\text { Standard }\end{array}$ & $\begin{array}{l}\text { STARLAB GmbH, Ahrensburg, } \\
\text { Germany }\end{array}$ \\
\hline T4 ligase & $\begin{array}{l}\text { New England Biolabs GmbH, Frankfurt } \\
\text { am Main, Germany }\end{array}$ \\
\hline Taq DNA polymerase & $\begin{array}{l}\text { New England Biolabs GmbH, Frankfurt } \\
\text { am Main, Germany }\end{array}$ \\
\hline Western Plus-ECL & PerkinEImer, USA \\
\hline Waymouth's MB 752/1 medium & $\begin{array}{l}\text { Thermo Fischer Scientific, Waltham, } \\
\text { USA }\end{array}$ \\
\hline Ethanol (99.9\%) & Merck, Darmstadt, Germany \\
\hline $\begin{array}{l}\text { L-ARGININE:HCL (13C6, 99\%), CLM- } \\
\text { 2265-H-0.1, Arg6 (Arg M) }\end{array}$ & $\begin{array}{l}\text { Cambridge Isotope Laboratories, Inc., } \\
\text { USA }\end{array}$ \\
\hline $\begin{array}{l}\text { L-ARGININE:HCL (13C6, 99\%; 15N4, } \\
\text { 99\%), CNLM-539-H-0.1 (Arg H) }\end{array}$ & $\begin{array}{l}\text { Cambridge Isotope Laboratories, Inc., } \\
\text { USA }\end{array}$ \\
\hline
\end{tabular}




\begin{tabular}{|l|l|}
\hline $0.05 \%$ Trypsin-EDTA, Gibco & Gibco, Massachusetts, USA \\
\hline L-LYSINE:2HCL (4,4,5,5-D4, 96-98\%), & Cambridge Isotope Laboratories, Inc., \\
DLM-2640-0.25 (Lys M) & USA \\
\hline BigDye ${ }^{\text {TM }}$ Terminator v1.1 Cycle & Applied Biosystems, Massachusetts, \\
Sequencing Kit & USA \\
\hline
\end{tabular}

2.1.8. Inhibitors

\begin{tabular}{|l|l|l|}
\hline Inhibitor & Concentration & Supplier \\
\hline AZD4547 & $0.003 \mu \mathrm{M}-7.5 \mu \mathrm{M}$ & Selleckchem, Munich, Germany \\
\hline AZD5363 & $0.5 \mu \mathrm{M}-35 \mu \mathrm{M}$ & Selleckchem, Munich, Germany \\
\hline BGJ398 & $0.003 \mu \mathrm{M}-7.5 \mu \mathrm{M}$ & Selleckchem, Munich, Germany \\
\hline FRAX597 & $0.01 \mu \mathrm{M}-10 \mu \mathrm{M}$ & Selleckchem, Munich, Germany \\
\hline IPA-3 & $0.01 \mu \mathrm{M}-30 \mu \mathrm{M}$ & Selleckchem, Munich, Germany \\
\hline PF-562271 & $0.01 \mu \mathrm{M}-7 \mu \mathrm{M}$ & Selleckchem, Munich, Germany \\
\hline
\end{tabular}

\subsubsection{Disposals}

\begin{tabular}{|c|c|}
\hline Disposal & Supplier \\
\hline $10 \mathrm{~mL}$ and $50 \mathrm{~mL}$ tubes & Cellstar, greiner bio-one, Germany \\
\hline $10 \mu \mathrm{L}, 10 \mu \mathrm{L} \& 1000 \mu \mathrm{L}$ filter/tips & SARSTEDT, Nümbrecht, Germany \\
\hline 10CM culture dishes & Cellstar, greiner bio-one, Germany \\
\hline 12-well plates & Cellstar, greiner bio-one, Germany \\
\hline $1 \mathrm{~mL}, 5 \mathrm{~mL}, 10 \mathrm{~mL} \& 25 \mathrm{~mL}$ pipettes & SARSTEDT, Nümbrecht, Germany \\
\hline 24-well plates & Cellstar, greiner bio-one, Germany \\
\hline 6-well plates & Cellstar, greiner bio-one, Germany \\
\hline 96-well plates & Cellstar, greiner bio-one, Germany \\
\hline Cells scraper $25 \mathrm{CM}$ & SARSTEDT, Nümbrecht, Germany \\
\hline Cryopure & SARSTEDT, Nümbrecht, Germany \\
\hline Disposable syringe, $10 \mathrm{ml}$ & SARSTEDT, Nümbrecht, Germany \\
\hline Disposable syringe, $2 \mathrm{ml}$ Luer & SARSTEDT, Nümbrecht, Germany \\
\hline DNA LoBind Tubes & Eppendorf, Hamburg, Germany \\
\hline
\end{tabular}




\begin{tabular}{|l|l|}
\hline Muse $\AA$ Count \& Viability Assay kit & Merck, Darmstadt, Germany \\
\hline QIAGEN Plasmid Midi Kit & QIAGEN, Venlo, Netherlands \\
\hline QIAprep Spin Miniprep Kit & QIAGEN, Venlo, Netherlands \\
\hline T175 culture flasks & SARSTEDT, Nümbrecht, Germany \\
\hline T25 culture flasks & SARSTEDT, Nümbrecht, Germany \\
\hline T75 culture flasks & SARSTEDT, Nümbrecht, Germany \\
\hline Tissue embedding plastic cassettes & Merck, Darmstadt, Germany \\
\hline Trans-Blot $®$ Turbo ${ }^{\text {TM }}$ RTA Mini & Bio-Rad, Feldkirchen, Germany \\
\hline Tube 30ml, 84x30mm, PP & \\
\hline Tube 7ml, 50x16mm, PS & SARSTEDT, Nümbrecht, Germany \\
\hline 1.5 and 2 mL microtubes & SARSTEDT, Nümbrecht, Germany \\
\hline Microfilters, $0.2 \mu M, 0.45 \mu M$ & SARSTEDT, Nümbrecht, Germany \\
\hline
\end{tabular}

\subsubsection{Chemical reagents}

\begin{tabular}{|l|l|}
\hline Reagent & Supplier \\
\hline 2-Mercaptoethanol & Carl Roth GmbH \& Co. KG, Karlsruhe \\
\hline Bovine Serum Albumin (BSA) & Carl Roth GmbH \& Co. KG, Karlsruhe \\
\hline EDTA-disodium salt & Carl Roth GmbH \& Co. KG, Karlsruhe \\
\hline Ethidium bromide & Carl Roth GmbH \& Co. KG, Karlsruhe \\
\hline G418 (Geneticin) & InvivoGen, California, USA \\
\hline Glacial acetic acid & Merck, Darmstadt, Germany \\
\hline HEPES & Sigma-Aldrich Co., St. Louis, USA \\
\hline Isopropanol (100\%) & Merck, Darmstadt, Germany \\
\hline Kanamycin & Gibco, Massachusetts, USA \\
\hline LC-MS grade water & Carl Roth GmbH \& Co. KG, Karlsruhe \\
\hline Na4P2O7 & Carl Roth GmbH \& Co. KG, Karlsruhe \\
\hline NaCl & Carl Roth GmbH \& Co. KG, Karlsruhe \\
\hline Na-deoxycholate & Carl Roth GmbH \& Co. KG, Karlsruhe \\
\hline Naorthovandate & Sigma-Aldrich Co., St. Louis, USA \\
\hline Na-orthovandate & Carl Roth GmbH \& Co. KG, Karlsruhe \\
\hline
\end{tabular}




\begin{tabular}{|l|l|}
\hline N-ethylmaleimide & Carl Roth GmbH \& Co. KG, Karlsruhe \\
\hline NP-40 & Carl Roth GmbH \& Co. KG, Karlsruhe \\
\hline Ponceau S & $\begin{array}{l}\text { AppliChem GmbH, Darmstadt, } \\
\text { Germany }\end{array}$ \\
\hline $\begin{array}{l}\text { Roche complete protease inhibitor } \\
\text { cocktail (cOmplete }{ }^{\mathrm{TM}} \text { ) }\end{array}$ & Merck, Darmstadt, Germany \\
\hline Sodium Chloride (NaCl) & Carl Roth GmbH \& Co. KG, Karlsruhe \\
\hline Sodium dodecyl sulfate (SDS) & Carl Roth GmbH \& Co. KG, Karlsruhe \\
\hline Sodium fluoride (NaF) & Carl Roth GmbH \& Co. KG, Karlsruhe \\
\hline $\begin{array}{l}\text { Sodium pyrophosphate tetrabasic } \\
\text { decahydrate }\end{array}$ & Carl Roth GmbH \& Co. KG, Karlsruhe \\
\hline B-glycerophosphate & Carl Roth GmbH \& Co. KG, Karlsruhe \\
\hline TRIS & Carl Roth GmbH \& Co. KG, Karlsruhe \\
\hline TRIS-hydrochloride & Carl Roth GmbH \& Co. KG, Karlsruhe \\
\hline Tween $\AA^{20}$ & $\begin{array}{l}\text { AppliChem GmbH, Darmstadt, } \\
\text { Germany }\end{array}$ \\
\hline Urea & Sigma-Aldrich Co., St. Louis, USA \\
\hline $\begin{array}{l}\text { B-Glycerophosphate disodium salt } \\
\text { hydrate }\end{array}$ & Carl Roth GmbH \& Co. KG, Karlsruhe \\
\hline
\end{tabular}

\subsubsection{Software}

\begin{tabular}{|l|l|}
\hline Software/database & Supplier \\
\hline 3500 data collection software 3 & $\begin{array}{l}\text { Applied Biosystems, Massachusetts, } \\
\text { USA }\end{array}$ \\
\hline Adobe Photoshop 7.0 & Adobe Systems Inc. San Jose, USA \\
\hline Cistrome CRISPR Scan database & $\underline{\text { http://cistrome.org/SSC/ }}$ \\
\hline CRISPR tefor database & $\underline{\text { http://crispor.tefor.net/crispor.py }}$ \\
\hline Endnote X9 & Clarivate analytics, Philadelphia, USA \\
\hline Geneious prime 2019 & https://www.geneious.com \\
\hline Graphpad Prism 7 for windows & $\begin{array}{l}\text { GraphPad Software, La Jolla } \\
\text { California USA, www.graphpad.com" }\end{array}$ \\
\hline
\end{tabular}




\begin{tabular}{|l|l|}
\hline ImageJ 1.8 & $\begin{array}{l}\text { Image J, Schneider, C. A.; Rasband, } \\
\text { W. S. \& Eliceiri, K. W. (2012), "NIH } \\
\text { Image to ImageJ: 25 years of image } \\
\text { analysis", Nature methods 9(7): 671- } \\
675\end{array}$ \\
\hline Microsoft office 2016 & $\begin{array}{l}\text { Microsoft corporation, Washington, } \\
\text { USA }\end{array}$ \\
\hline Perseus 1.6.10.43 & $\begin{array}{l}\text { http://www.coxdocs.org/doku.php?id= } \\
\text { perseus:common:download_and_inst } \\
\text { allation }\end{array}$ \\
\hline ZHANG LAB Guide Design Resources & $\begin{array}{l}\text { https://zlab.bio/guide-design- } \\
\text { resources }\end{array}$ \\
\hline
\end{tabular}




\subsection{Methods}

\subsubsection{Patients tissue samples}

\subsubsection{Tissue samples collection}

Tissue samples were collected from lung cancer patients through surgical resections. Resections were held by Department of Thoracic Surgery at the University Medical Center of Goettingen, Germany. The collection and usage of patient tissue samples were approved by the Ethics Committee at the University Medical Center of Goettingen (\#1-2-08). All patients included in this study have been informed and given consent. Collection of samples and experiments have been carried out according to declaration of Helsinki as well as institutional, state and federal guidelines.

\subsubsection{Cell block}

Cell blocks were prepared according to a published protocol (Varsegi \& Shidham, 2009). Cell lines were seeded in T-175 culture flasks and harvested when they reached $80 \%$ confluency. Cells were washed once with PBS, centrifuged at 2000 RPM for five minutes and placed in a flat bottom $7 \mathrm{~mL}$ glass tube (small tube) inside a $30 \mathrm{~mL}$ plastic tube (carrier tube) then centrifuged once again at 2000 RPM for five minutes. HistoGel (Thermo Scientific) was prepared through heating for 30 seconds in microwave. Cell pellets were re-suspended in $1 \mathrm{~mL}$ warm Histogel. One piece of AV-marker was immersed in each gel/cells sample in order to mark the location and layer (depth) of cells inside the blocks. AV-marker is used in cell blocks preparation in order to detect the location and depth of cell pellets while cutting the paraffin blocks. Markers were prepared as described before (Varsegi \& Shidham, 2009). Shortly, AVmarkers were set up through collecting small pieces of raw banana peels with dimensions of about $2 \times 2 \times 2 \mathrm{~mm}$ then immersing them in black India ink. Banana peels were incubated in ink for ten minutes and then were washed using $10 \%$ formalin. Markers were stored in $10 \%$ formalin at room temperature until usage. Carrier tubes were filled with $5 \mathrm{~mL}$ warm water $\left(45^{\circ} \mathrm{C}\right)$, which has surrounded the small tubes and prohibited the solidification of gel through next steps. Tubes were centrifuged at 2000 RPM for five minutes. Small tubes were carefully removed out of the carrier tubes and placed in fridge at $4{ }^{\circ} \mathrm{C}$ for one hour in order to let the gel solidify. Afterwards, gels were removed from the small tubes via injecting water underneath gels using a 
syringe. Gels were placed in labelled plastic cassettes, fixed in formalin overnight and embedded in paraffin.

\subsubsection{Immunohistochemistry (IHC)}

Immunohistochemistry was used to evaluate expression levels of FGFR1 protein and phosphorylation levels of Akt in lung cancer tissue samples and cell blocks. The technique was performed as published before (Bohnenberger et al., 2018). For patient tissue samples, tissue microarrays embedded in paraffin were stained, while paraffin embedded cell blocks were used to stain cell lines. Paraffin sections of $2 \mu \mathrm{m}$ thickness were cut from paraffin blocks then incubated with EnVision Flex Target Retrieval Solution from Dako at high PH value for FGFR1 and pAkt staining (PH=9). Afterwards, sections were incubated with primary antibody (anti-FGFR1, dilution: 1:5000, Abcam or anti-pAkt 1:100 Abcam) for 20 minutes at room temperature. A secondary antibody conjugated to HRP was used to the primary antibody. HRP was visualized by DAB substrate. Mayer's Haematoxylin staining was used for contrasting. Samples were evaluated under light microscopy. Intensity of the staining has divided samples into three groups of zero=no expression, one $=$ weak expression and two $=$ strong expression.

\subsubsection{Florescence in situ hybridization (FISH)}

Florescence in situ hybridization or FISH was used to check for FGFR1 gene amplification in lung cancer patient tissues as well as in lung cancer cell lines. FISH staining and analysis were carried out as described before (Schildhaus et al., 2012). For patient tissues, samples were fixed in formalin then embedded in paraffin blocks then re-arranged on tissue microarray blocks. For cell lines, cell blocks were fixed in formalin then embedded in paraffin blocks. Paraffin blocks were cut into 3-4 $\mu \mathrm{m}$ thickness sections then mounted on slides. VP2000 processor system was used for the deparaffinization, protease treatment and washing steps of the slides. Slides are then denatured at $75^{\circ} \mathrm{C}$ and hybridized with ZytoLight SPEC FGFR1/CEN8 probe at $37^{\circ} \mathrm{C}$ overnight. Afterwards, slides were stained with DAPI.

Positive control cells as non-tumor lung tissue or fibroblasts had to display at least one signal of each color to evaluate the samples. For evaluation, at least 60 nuclei 
from the green stained FGFR1 or the orange stained centromere 8 (CEN8) were analyzed. The sample was evaluated as positive for amplification if the ratio between FGFR1 signals and CEN8 signals in one cancer cell nucleus was equal to or larger than two, average green signals in one nucleus were larger than or equal to six or $10 \%$ or more of the cancer cells have shown 15 or more green signals or large clusters.

\subsubsection{Cell culture}

\subsubsection{Cell lines}

In this study, 21 human cell lines of lung cancer from different histological groups were used. The properties and sources of cell lines are listed in materials section (chapter 2.1.3). All the cell lines were cultured using RPMI 1640, Dulbecco Modified Eagle Medium (DMEM) or Waymouth's MB growth media. All media included 10\% fetal bovine serum (FBS), 1\% glutamine and 1\% Penicillin/Streptomycin. Cells were incubated at $37{ }^{\circ} \mathrm{C}$ and $5 \% \mathrm{CO}_{2}$ incubators. Cells were splitted every three days and kept between $10 \%$ and $80 \%$ confluency. For cells detachment, $0.05 \%$ Trypsin-EDTA was used.

\subsubsection{Cryopreservation of cell lines}

Cells were cultured until appropriate density then detached from flasks using $0.05 \%$ Trypsin-EDTA (in case of adherent cells). Cells were centrifuged at 1400 RPM, resuspended in freezing medium (5\% DMSO in FCS) and then kept for 24 hours in freezing boxes (Mr. Frosty) at $-80^{\circ} \mathrm{C}$. Cell lines were preserved in liquid nitrogen for long time or in $-80^{\circ} \mathrm{C}$ freezer for short time preservation.

\subsubsection{Western blot}

To collect protein lysates, cells were collected, centrifuged at 1400 RPM and then incubated with NP-40 lysis buffer on ice for 20 minutes. Cell lysates were collected from the supernatant face after the second centrifugal step (at 14,000 RPM for 20 minutes). In order to load equal amounts of cell lysates, concentrations of lysates were determined using DC protein assay kit and read on plate reader (TECAN 200M pro). Equal amounts of lysates were denatured using SDS loading buffer at $95{ }^{\circ} \mathrm{C}$ for 
five minutes. Lysates were loaded on mini-protean precast gels (4-20\%). Proteins were separated using voltage between 70 and 120 volts. Proteins were transferred from gels to nitrocellulose membranes using Trans-Blot Turbo. Membranes were then blocked using 5\% milk-TBST buffer for one hour at room temperature and incubated with primary antibodies either for two hours at room temperature or overnight at $4{ }^{\circ} \mathrm{C}$. Membranes were washed three times for ten minutes each using TBST buffer then incubated with the appropriate secondary antibody for one hour at room temperature. Membranes were washed three times for ten minutes each. Reincubation with primary antibodies was feasible through five minutes incubation with Reblot Plus Strong antibody stripping solution at room temperature. Signals were developed using Western Plus-ECL and Fusion Fx, peQlab camera.

\subsubsection{MTS assay}

MTS assay is a colorimetric assay, which depends on reduction of MTS tetrazolium compound through the NADPH dehydrogenase enzyme in living cells. Only viable cells can reduce tetrazolium compound to the soluble formazan with a specific color, which can be detected using plate readers at absorbance between 490 and $500 \mathrm{~nm}$ (Aslantürk, 2018). For the assay, cells were seeded in $200 \mu \mathrm{l}$ cell culture medium in 96-well plates with the appropriate density and then incubated with either an inhibitor or DMSO for inhibition assays or with either specific or control siRNA for proliferation assays. After the appropriate incubation time, $20 \mu \mathrm{L}$ of MTS reagent (Cell titer aqueous one solution) were added to the cells and incubated for three hours at 37 ${ }^{\circ} \mathrm{C}$. Intensity of formazan dye was measured on TECAN 200m Pro plate reader (wavelength $=660 \mathrm{~nm}$ ). Results were controlled through comparison to DMSO treatment, day one or control siRNA in case of inhibition assays, proliferation assays or siRNA knockdown, respectively.

\subsubsection{Cell counting and viability using Guava $\AA$ Muse ${ }^{\circledR}$ cell analyzer}

Viable cells were counted using Guava Muse cell analyzer. Briefly, cells were detached using $0.05 \%$ EDTA-trypsin. Trypsin interaction was stopped using equal amounts of complete growth medium. Next, cells were collected in $15 \mathrm{~mL}$ falcon tubes and centrifuged for five minutes at speed of 1400 RPM. Supernatant was 
discarded and cells were re-suspended in $3 \mathrm{~mL}$ growth medium. Appropriate volume of cell suspension ( $20 \mu \mathrm{L}$ or $50 \mu \mathrm{L}$ depending on cells concentration) was mixed with count and viability reagent $(380 \mu \mathrm{L}$ or $450 \mu \mathrm{L})$ and incubated for five minutes at room temperature. Viable cell number and concentration were measured using Muse cell analyzer.

\subsubsection{PI viability assay}

Propidium iodide (PI) stain was used to detect percentage of viable cells. Cells were incubated with the appropriate inhibitor or DMSO for the recommended duration. At the time of analysis, growth medium was removed, and cells were harvested using $0.05 \%$ trypsin-EDTA. Cells were washed one time with PBS, moved to FACS tubes and then washed once again and centrifuged for five minutes at speed of $300 \mathrm{~g}$. For each sample, $7 \mu \mathrm{L}$ of $10 \mu \mathrm{g} / \mathrm{mL}$ PI stain was added and incubated for ten minutes at room temperature and then analyzed using BD flow cytometer.

\subsubsection{Cloning}

\subsubsection{Antibiotic killing curves}

Antibiotic killing curves were used to determine the lowest concentration of the antibiotic, which can lead to death of cell lines. Transfection vectors had antibiotic resistance genes, which led to survival of successfully transfected cell lines due to antibiotic resistance gene on the transfected plasmid. Cell lines were incubated with elevated concentrations of Neomycin (G418) (100-1000 $\mu \mathrm{g} / \mathrm{mL})$ in normal growth medium for one week. The lowest concentration, which led to the complete death of cells after seven days was chosen as the appropriate selection concentration for this cell line.

\subsubsection{Expression vectors}

Protein kinase B / Akt1 was constitutively activated in different lung cancer cell lines using human expression vectors. pcDNA3 Myr-Akt1 (Akt vector) as well as pcDNA3 Flag HA (control vector) plasmids were obtained from Addgene company. The pair of plasmids were designed with neomycin resistance genes for selection. Plasmids were seeded on agar plates with ampicillin $(100 \mu \mathrm{g} / \mathrm{mL})$ and single clones were 
picked after 18 hours. Clones were expanded in LB growth medium with ampicillin and then purified using plasmid maxiprep kit from Qiagen according to the manufacture's protocol. $2.5 \mu \mathrm{g}$ of Akt1 expression vector or pcDNA3 empty vector were complexed with $7 \mu \mathrm{g}$ of Lipofectamin3000 transfection reagent in $500 \mu \mathrm{L}$ serum free medium. Complexing mixtures were incubated for ten minutes at room temperature. Transfection mixtures were added drop wise to the corresponding cells seeded in 6-well plates. Successfully transfected cells were selected using Geneticin antibiotic (G418) according to cells' antibiotic killing curves. The complete death of non-transfected cells was taken as a proof of successful transfection. Expression levels of phosphorylated Akt were evaluated using western blotting.

\subsubsection{Knockdown using siRNA}

Fibroblast growth factor receptor 1 was knocked down in different lung cancer cell lines using siRNA. Two functionally verified siRNAs against FGFR1 gene were purchased from Qiagen Ltd. The two siRNAs had catalogue numbers of SI02224684 andSI02224677. The specific effect of FGFR1-siRNAs was controlled using a scrambled control siRNA from the same company (Allstars negative control siRNA ()). All siRNAs, including FGFR1-targeted and controls, were used at a concentration of $30 \mathrm{nM}$ and were transfected using Hiperfect transfection reagent. All starts control siRNA with a modification of Alexa Fluor 488 florescence color was used to control the transfection efficiency of siRNA in targeted cells using FACS machine. The general protocol of siRNA preparation included dilution of siRNA at the appropriate concentration using serum-free growth medium. Next, siRNAs were incubated with the transfection reagent Hiperfect for ten minutes at room temperature to allow formation of transfection lipid vesicles. Transfection mixture was added drop wise on top of cells then incubated in $37^{\circ} \mathrm{C}$ incubator. For immunoblotting of knocked down cell lines, cells were seeded in 6-well plates, treated with siRNAs and then harvested and lysed after 48 hours. For proliferation assays, cell lines were seeded in 96-well plates in triplicates and then treated, incubated and measured every 24 hours for six days. 


\subsubsection{CRISPR/Cas9 knockout}

In this project, CIRSPR/Cas9 was used to knockout FGFR1 gene in the SQCLC H1703 cell line. Two gRNAs (5'TTCCCAGGTCCCCTAAGAGG3' \& 5'GGAGCACCAGTGTAGCCAGG3') have been designed using Zhang lab and Cistrome online tools. The gRNAs were designed to target introns up and down stream of exon 14 in the FGFR1 gene. One of the two primers was cloned in a green florescence protein (GFP)-Cas9 vector backbone (no. PX458, Addgene), while the other primer was cloned in mCherry-Cas9 backbone (no. 64217, Addgene). Cloned primers were transformed into DH5alpha competent bacteria and let to grow on agar plates with ampicillin at $37{ }^{\circ} \mathrm{C}$ overnight. Bacterial clones were picked up and expanded in LB medium with ampicillin for another 18 hours then harvested and purified using Qiagen maxiprep. The success of cloning was verified through sequencing of Cas9-backbones using U6 primer (5'GAGGGCCTATTTCCCATGATTCC3'). Successfully cloned primers from both GFP and mCherry vectors were diluted in serum-free medium alongside with P3000 transfection reagent in two different tubes. Lipofectamine3000 transfection reagent was diluted in serum-free medium in another tube. The two reagents and DNA were mixed and allowed to complex for ten minutes at room temperature to form lipid transfection vesicles.

Transfection mixture was added dropwise on the cells in 6-well plates and incubated at $37^{\circ} \mathrm{C}$ incubator. After 18 hours, growth medium was changed and successful double transfection was checked using ZOE fluorescent cell imager microscope. After 48 hours, double transfected cells were sorted into single cells on 96-well plates using BD FACS sorter. Three weeks after sorting, single growing clones have been marked on the 96-well plates. Five weeks after sorting, clones were transferred to 24well plates and subsequently 6-well plates. Finally, clones were tested for the success of knockout using western blot and DNA sequencing.

\subsubsection{DNA extraction}

For DNA extraction, Qiagen DNA extraction kit was used. DNA was extracted according to the manufacture's protocol. Cells were lysed with $300 \mu \mathrm{L}$ cell lysis solution with $10 \mu \mathrm{L}$ Proteinase $\mathrm{K}$ and then incubated at $56{ }^{\circ} \mathrm{C}$ for 30 minutes. Afterwards, $1.5 \mu \mathrm{L}$ Rnase A solution was added and incubated with lysates for 30 
minutes at $37^{\circ} \mathrm{C}$. One hundred $\mu \mathrm{L}$ protein precipitation solution was added to the lysates with vortex of 20 seconds. Lysates were centrifuged at 14000 RPM for three minutes at room temperature. Supernatant was moved in new tubes, washed with $300 \mu \mathrm{L} 100 \%$ isopropanol and then centrifuged again at $14000 \mathrm{RPM}$ for three minutes at room temperature. Pellets were washed with $300 \mu \mathrm{L} 70 \%$ ethanol and centrifuged for five minutes at 14000 RPM at room temperature. Supernatant was removed, pellets were let to dry and then re-suspended in 20-50 $\mu \mathrm{L}$ RNase free water.

\subsubsection{Sanger sequencing}

DNA sequencing of plasmids or cell lines was carried out according to BigDye ${ }^{\mathrm{TM}}$ Terminator v3.1 sequencing kit protocol. For cell lines, an initial step included amplification of target DNA sequence using polymerase chain reaction (PCR) followed by a cleaning step using ExoSAP clean up kit. For plasmids, purified DNA of plasmids was used directly without additional cleaning steps. DNA sequencing included three main steps: PCR reaction using BigDye ${ }^{\mathrm{TM}}$ Terminator v1.1 Cycle Sequencing Kit, cleaning step using BigDye XTerminator ${ }^{\mathrm{TM}}$ purification kit and reading step performed using 3500 DX Series Genetic Analyzer from ThermoFisher Scientific. Results of DNA sequencing were analyzed using Geneiousprime 2019 software.

\subsubsection{Library preparation for Archer FusionPLEX CTL sequencing panel}

An amount of 200-500 ng of DNA was extracted from cell lines. DNA was then sheared using a focused-ultrasonicator into fragment sizes of around $300 \mathrm{bp}$. The NEBNext Ultra II DNA library was used to prepare the sequencing libraries. Average reads developed per each sample was 17.1 million reads. The human reference genome (HG19) was used to map the sequences using version 0.7.12 of BWA.

\subsubsection{Phosphoproteomic Mass spectrometry analysis}

\subsubsection{SILAC metabolic labeling}

Complete SILAC medium was prepared using SILAC growth medium depleted of arginine and lysine amino acids, $10 \%$ of dialyzed FBS which has significantly low 
concentrations of small molecules like amino acids, medium weight arginine (13C6) and medium weight lysine $(4,4,5,5-\mathrm{D} 4)$ or heavy weight arginine $(13 \mathrm{C} 6,15 \mathrm{~N} 4)$ and heavy weight lysine (13C6, 15N2), Na-pyruvate, glutamine and penicillinstreptomycin antibiotics. To achieve high level of heavy amino acids incorporation, cells were incubated for at least ten cell divisions in SILAC medium at $37^{\circ} \mathrm{C}$ and $5 \%$ $\mathrm{CO}_{2}$ incubator. Prior to lysing, medium SILAC labelled cells were treated with DMSO and heavy labeled cells with $0.5 \mu \mathrm{M}$ AZD4547 for three hours.

\subsubsection{Lysing SILAC labelled cells}

SILAC labelled cells were washed once with ice-cold PBS to remove residuals of growth medium and to stop cellular interactions. Washing was followed by scraping cells on ice-cold PBS using $25 \mathrm{~cm}$ cell scrapper followed by centrifugation of cells (1300 rpm, 5 min. at room temperature). Cell pellets were divided into two groups for global proteome and pyome analysis with a ratio of $1: 2$.

Global proteome pellets were lysed using global proteome NP40 lysis buffer (described in materials section: 2.1.6) for 20 minutes on ice. Cell pellets were centrifuged at 20,000 RPM for 20 minutes at $4{ }^{\circ} \mathrm{C}$. Lysates were collected from supernatant layer and moved to Eppendorf lobind tubes. Lysates' concentration was measured using Pierce ${ }^{\mathrm{TM}}$ BCA Protein Assay Kit followed by snap freezing in liquid nitrogen. Lysates were kept at $-80^{\circ} \mathrm{C}$.

Pyome cell pellets were lysed using urea lysis buffer (described in materials section: 2.1.6) for 20 minutes at room temperature. Pyome lysates were then sonicated using $15 \mathrm{~W}$ output with 1-2 bursts for ten seconds each. Cell pellets were then centrifuged at speed of 20,000 RPM for 20 minutes at room temperature. Lysates were collected from the upper phase into $15 \mathrm{~mL}$ falcon tubes. Concentration of lysates was tested through Pierce ${ }^{\mathrm{TM}}$ BCA Protein Assay. Pyome lysates were snap frozen using liquid nitrogen then held in $-80^{\circ} \mathrm{C}$ freezer.

\subsubsection{Mass spectrometry sample preparation}

Preparation of LC-MS/MS samples followed the previously published protocols (Corso et al., 2016; Mohr et al., 2017). To enrich peptides for global phosphorylation, equal amounts of lysates labelled with SILAC medium were mixed together then 
reduced using $10 \mathrm{mM}$ DTT for 1 hour at $65^{\circ} \mathrm{C}$ and alkylated using $20 \mathrm{mM}$ IAM for 1 hour at $37^{\circ} \mathrm{C}$. Afterwards, trypsin was used to digest the lysates. Trypsin was used at concentration of $1: 20$ to $1: 50$ weight/weight of trypsin: protein at $37{ }^{\circ} \mathrm{C}$ in $0.1 \%$ RapiGest. Digested peptides were then acidified using 1\% formic acid and centrifuged for 30 minutes at maximum speed. To enrich peptides for global phosphorylation, fractions of GPome cell lysates were applied to TiO2 columns, washed three times on spin columns, eluted using $0.5 \mathrm{M} \mathrm{NH} 4 \mathrm{OH}$, desalted on $\mathrm{C} 18$ spin column and dried in a speed vacuum centrifuge.

To enrich peptides for tyrosine phosphorylation, pYome cell lysates were mixed together in equal ratios of light, medium and heavy SILAC media labelled lysates. Next, PTMScan® Phospho-Tyrosine (P-Tyr-1000) Rabbit mAb Kit was used according to the manufacturer procedure and the previously published protocols (Rush et al., 2005). Briefly, tyrosine phosphorylated peptides were purified through their immunological affinity to tyrosine antibodies, which were conjugated to agarose beads then washed and eluted with a diluted acid. Peptides were then fractionized using strong cation exchange (SCX) as described before (Mohr et al., 2017).

Next, samples were loaded on high performance liquid chromatography (HPLC) column using $0.1 \%$ formic acid and $80 \%$ acetonitrile with $0.1 \%$ formic acid as buffers $A$ and $B$, respectively. HPLC system (temperature set at $50{ }^{\circ} \mathrm{C}$ ) was adjusted on a flow rate of $300 \mathrm{~nL}$ per minute. Liquid chromatography system was coupled online to Q Exactive ${ }^{T M}$ HF Hybrid Quadrupol-Orbitrap mass spectrometer. The data dependent acquisition and automatic switch between first and second scans (MS and MS/MS) were chosen. Precursor ions of charges between 2 and 5 were selected for the second MS. Raw MS data was analyzed with MaxQuant according to the published protocol (Cox \& Mann, 2008). Andromeda search engine was used to map the quantified sites to Uniprot human database. False discovery rate (FDR) was set to $1 \%$. Perseus analysis tool was used to test quality and annotation of the quantified sites.

\subsubsection{Perseus phosphoproteomic data analysis}

To analyze phosphoproteomic data, I used Perseus software version number 1.6.10.43. Data analysis followed the general scheme of work described by 
developers (Tyanova \& Cox, 2018; Tyanova et al., 2016). Firstly, I filtered the sites for potential contaminants and reverse recognized peptides. Then, site table was expanded to combine multiple phosphorylated sites followed by checking normal distribution of data after transforming it into log2. Multi-scatter plots were used to calculate the $R$ values, which show the similarities between replicates in each sample. Data was filtered according to localization probability $>0.75$. Samples were renamed and grouped together followed by calculating the average of technical replicates. Statistical analyses were carried out using different biological replicates for each sample. To generate heatmaps, data were z-scored as well as compensated for missing values. Heatmaps were generated to compare different groups based on all phosphosites as well as ANOVA+ significant enriched phosphosites. Volcano plots were generated using student t-test to compare each sample to the control sample.

\subsubsection{Statistical analysis}

Correlations between pathological features of patients and FGFR1 gene amplification or protein expression were carried out using Person's coefficient, Chi-square test and Contingency test. Overall survival of patients was correlated to amplification or expression using Kaplan-Meier analysis. P-values were calculated according to Mantel-Cox, Chi-square and student $t$ tests. $P$-values of less than or equal 0.05 were considered statistically significant. Combination indexes $(\mathrm{Cl})$ were calculated according to Chou-Talalay method. CompuSyn software was used to calculate $\mathrm{Cl}$ values, which identified the nature of multiple drug interaction. $\mathrm{Cl}<1, \mathrm{Cl}=1, \mathrm{Cl}>1$ indicate synergistic, additive and antagonistic effects, respectively (Chou, 2010). 


\section{Results}

\subsection{Studying prevalence and correlation of FGFR1 gene amplification and protein expression in lung cancer patients}

\subsubsection{FGFR1 amplification as molecular target in squamous cell lung cancer}

Fibroblast growth factor receptor 1 (FGFR1) gene amplification is a potential molecular target in squamous cell lung cancer (SQCLC). In order to study the relevance of FGFR1 amplification as a molecular target, prevalence of FGFR1 gene amplification has to be established among lung cancer patients. The first aim of the project was to examine and validate FGFR1 gene amplification as well as protein expression levels among lung cancer patients. Collection of patient samples and their immunohistochemical diagnosis were done in collaboration with Anna-Maria Lois, Institute of Pathology, University Medical Center of Goettingen, Germany. Gene amplification diagnosis of patient samples was done in collaboration with group of Prof. Dr. Hans-Ulrich Schildhaus, institute of Pathology, University Medical Center of Goettingen, Germany.

In order to test FGFR1 prevalence, a cohort study consisted of 421 lung cancer patient samples was established. The cohort included 208 patients (49.4\%) diagnosed with squamous cell lung cancer (SQCLC), 121 patients (28.7\%) diagnosed with pulmonary adenocarcinoma (AC) and 45 patients (29.9\%) diagnosed with small cell lung cancer (SCLC). Males formed $70.1 \%$ of the cohort, while females formed $29.9 \%$ of the cohort. The median age of patients was 66 years with range of 34 to 85 years. Tissue samples were collected through surgical resections at different stages of the tumor. Stages of lung cancer were classified and assigned according to TNM classification of the Union for International Cancer Control (UICC) edition number 7 . Stage I formed $47.7 \%$ of the group followed by $25.9 \%, 19 \%$ and $1.9 \%$ for stages II, II and IV, respectively. The follow-up time had a median of 30 months and ranged between 1 and 196 months. During the follow-up time, 227 patients deceased while 166 were alive. General histopathological and clinical features of patients are summarized in table 2. 
Table 2: Lung cancer patient characteristics.

\begin{tabular}{lcccc}
\hline Feature & Cases & AC & SQCLC & SCLC \\
\hline $\begin{array}{l}\text { Total } \\
\text { Age median (range) }\end{array}$ & 374 & 121 & 208 & 45 \\
Gender & $66(34-85)$ & $67(34-85)$ & $66(42-83)$ & $67(34-81)$ \\
$\quad$ Male & $277(74.1 \%)$ & $67(24.2 \%)$ & $177(63.9 \%)$ & $33(11.9 \%)$ \\
$\quad$ Female & $97(25.9 \%)$ & $54(55.7 \%)$ & $31(31.9 \%)$ & $12(12.4 \%)$ \\
Degree of differentiation & & & & \\
$\quad$ II & $249(66.6 \%)$ & $89(35.7 \%)$ & $160(64.3 \%)$ & $0(0 \%)$ \\
$\quad$ III & $125(33.4 \%)$ & $32(25.6 \%)$ & $48(38.4 \%)$ & $45(36 \%)$ \\
Lymph node metastasis & & & & \\
$\quad$ Yes & $139(39.5 \%)$ & $42(30.2 \%)$ & $85(61.2 \%)$ & $12(8.6 \%)$ \\
$\quad$ No & $213(60.5 \%)$ & $70(32.8 \%)$ & $119(55.9 \%)$ & $24(11.3 \%)$ \\
Clinical stage & & & & \\
$\quad$ I+II & $270(75.8 \%)$ & $92(34.0 \%)$ & $150(55.6 \%)$ & $28(10.4 \%)$ \\
$\quad$ III+IV & $86(24.2 \%)$ & $26(30.2 \%)$ & $57(66.3 \%)$ & $3(3.5 \%)$ \\
Resection status & & & & \\
$\quad$ R1+2 & $330(90.4 \%)$ & $109(33.0 \%)$ & $188(57.0 \%)$ & $33(10.0 \%)$ \\
$\quad 35(9.6 \%)$ & $8(22.9 \%)$ & $20(57.1 \%)$ & $7(20.0 \%)$ \\
\hline
\end{tabular}

AC: adenocarcinoma, SQCLC: squamous cell lung cancer and SCLC: small cell lung cancer.

\subsubsection{FGFR1 gene amplification in SQCLC and SCLC samples}

Amplification of the FGFR1 gene, located on chromosome 8, was diagnosed using fluorescence in situ hybridization (FISH). SQCLC as well as SCLC tissue samples were hybridized with green fluorescent and orange fluorescent probes against FGFR1 gene and centromere 8 (CEN8), respectively. Ratios of FGFR1/CEN8 (green/red) signals were calculated. Amplification was accredited if the ratio between FGFR1 signals and CEN8 signals in one cancer cell nucleus was equal to or larger than two, average green signals in one nucleus were larger than or equal to six or $10 \%$ or more of the cancer cells showed 15 or more green signals or large clusters (Figures 5A and B). 

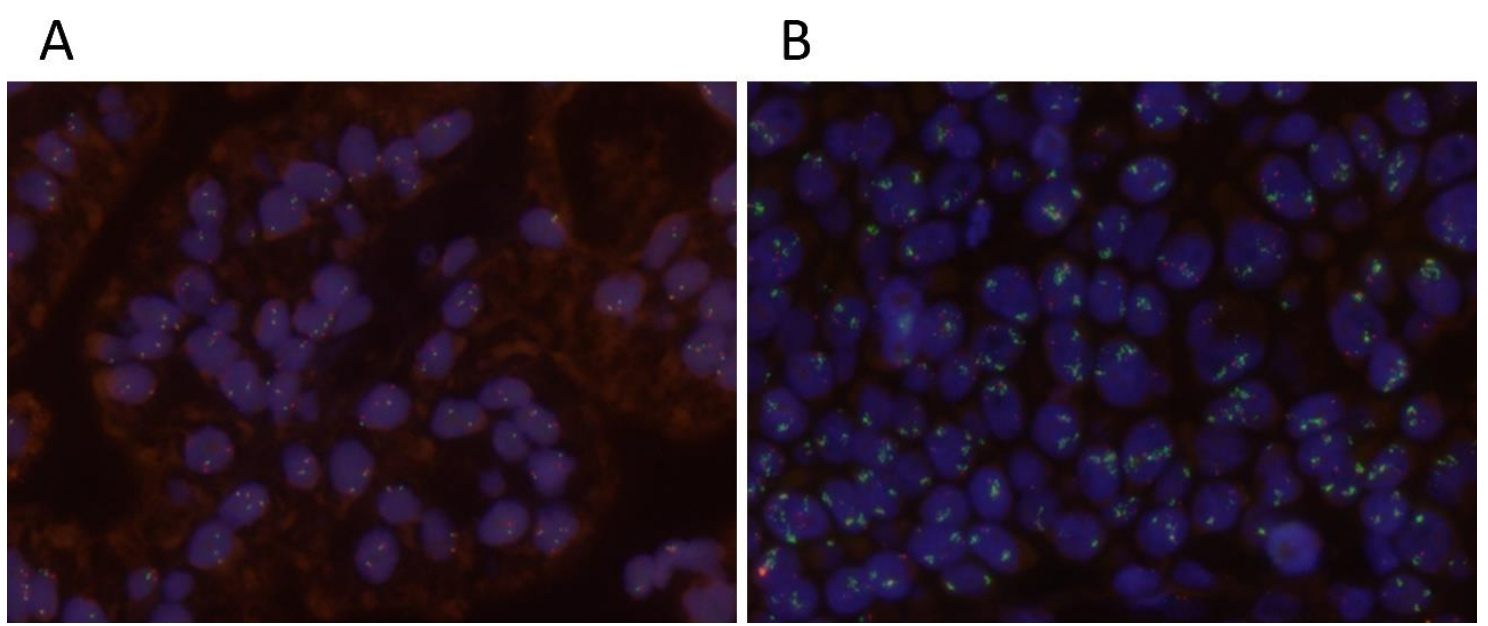

Figure 5: FGFR1 gene amplification in tissue samples of lung cancer patients.

FISH analysis of FGFR1 gene amplification in an amplified sample (A) and a non-amplified sample (B). Green signals indicated FGFR1 gene, while red signals indicated centromere 8. Modified from: (Elakad et al., 2020).

FISH analysis showed amplification of FGFR1 gene in 37 out of 156 (23\%) evaluable SQCLC samples. In the SCLC group, FGFR1 gene was amplified in 3 out of $37(8 \%)$ evaluable samples (Figure 6).

\section{FGFR1 amplification prevalence}

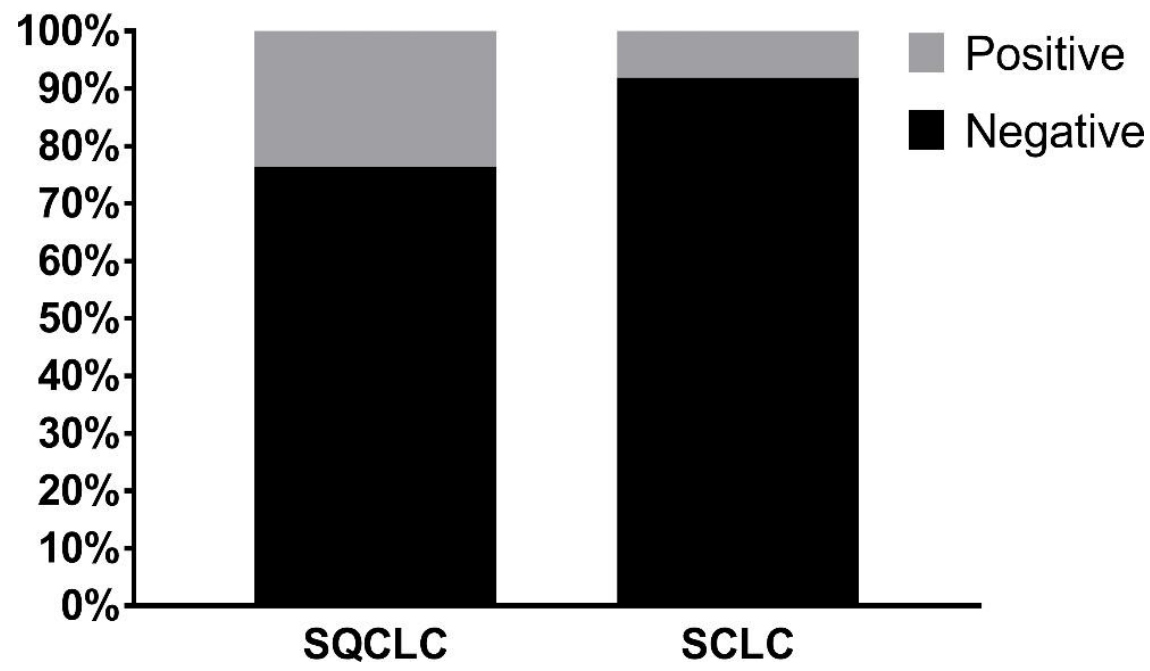

Figure 6: Prevalence of FGFR1 gene amplification in lung cancer patient tissue samples.

FISH analysis detected prevalence of FGFR1 gene amplification in SQCLC and SCLC patient samples. Modified from: (Elakad et al., 2020).

In order to test if FGFR1 gene amplification status can be used as a prognostic factor for SQCLC or SCLC patients, I compared patients' overall survival to the results of 
FISH analysis. Kaplan-Meier analysis did not show any significant correlation between the two factors in SQCLC ( $p=0.73, \mathrm{HR}=0.92$ and $95 \% \mathrm{Cl}=0.58-1.45)$ or SCLC $(p=0.69 H R=1.4$ and $95 \% \mathrm{Cl}=0.26-7.51)$ (Figures $7 \mathrm{~A}$ and $\mathrm{B})$.

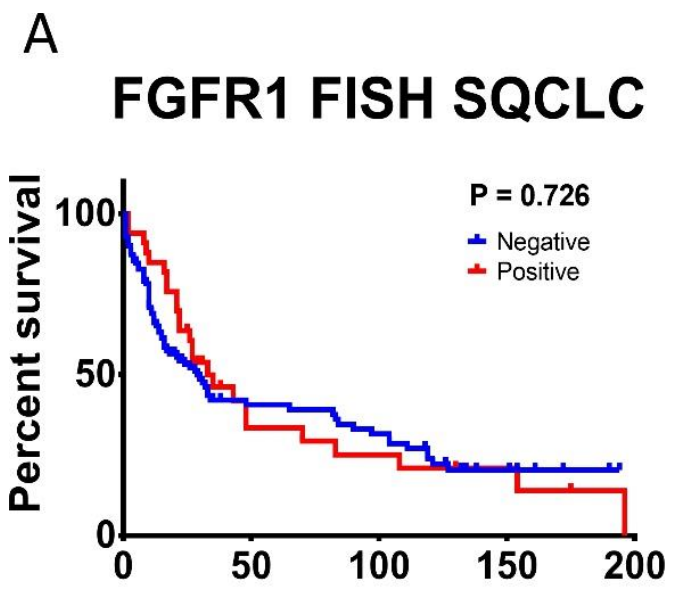

Overall survival time (months)
B FGFR1 FISH SCLC

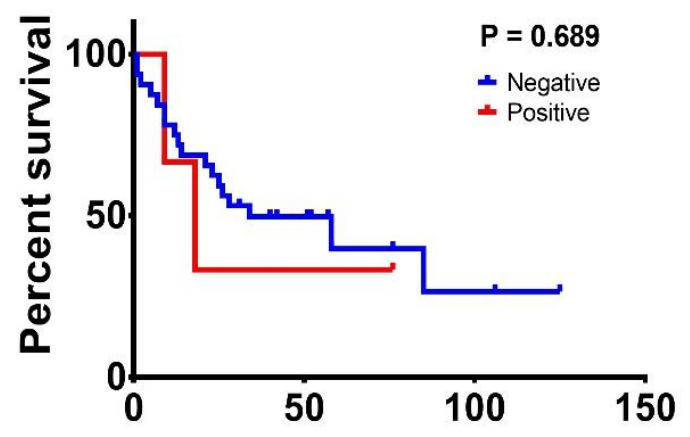

Overall survival time (months)

Figure 7: Survival analysis according to FGFR1 amplification in lung cancer patients.

Kaplan-Meier curves compared survival in amplified vs. non-amplified patient samples in SQCLC (A) and SCLC (B). P-values were calculated according to Mantel-Cox Chi-square test. Modified from: (Elakad et al., 2020).

\subsubsection{Validation of immunostaining}

Immunohistochemistry (IHC) staining is prone to false positive or negative results due to off-target binding. In order to validate specificity and accuracy of the anti-FGFR1 antibody as well as the used protocol, I used two different methods. First, I stained normal human tonsil and gallbladder tissue samples. Protein expression levels as well as RNA levels in these tissues are well established and validated. Results of IHC showed that tonsil tissue was a weakly positive tissue for FGFR1 expression while gallbladder tissue was a strong expressing tissue. These results correlated to RNA and protein expressions published by proteinatlas.org, FANTOM5 and HPA databases (Figures 8A and B). (Lizio et al., 2015; Uhlen et al., 2015) 
A

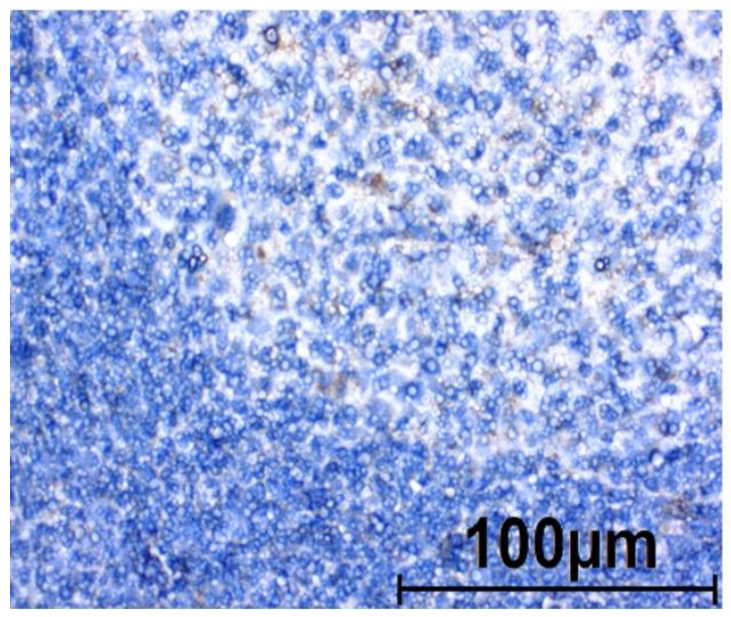

B

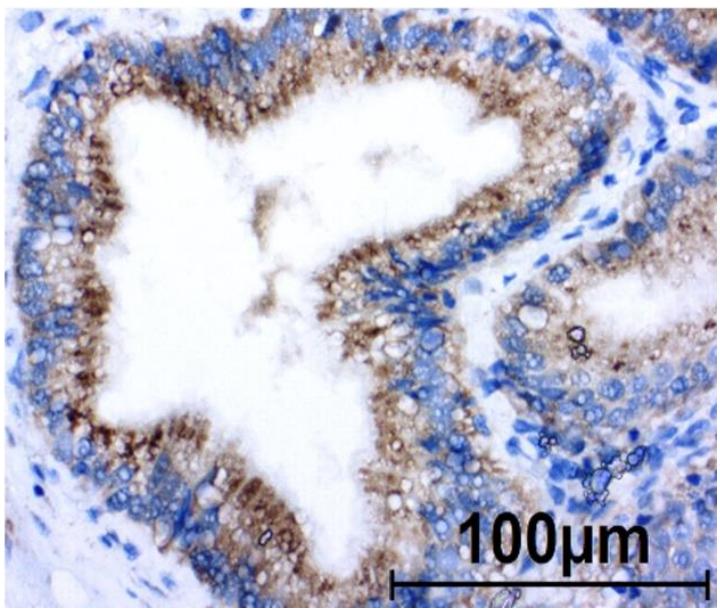

Figure 8: Validation of anti-FGFR1 antibody in human tissue samples.

Immunohistochemistry staining of human healthy tonsil tissue (A) and gallbladder tissue (B). All images were captured at 40x magnification. Modified from: (Elakad et al., 2020).

Secondly, I used the CRISPR/Cas9 system to knock out the FGFR1 gene in a human lung cancer cell line (H1703). The cell line H1703 is a SQCLC cell line, which normally expresses high level of FGFR1 protein. Two different guide RNAs were designed to target introns upstream and downstream of exon number 14 leading to its complete deletion.

The targeted exon was chosen as it forms part of the vital intracellular kinase domain of the receptor. All 21 isoforms of FGFR1 protein include exon 14, which assured the disruption of all isoforms. Last characteristic of exon 14 is that it consists of 191 nucleotides, which is not divisible by three. This feature leads to a frameshift mutation in the downstream sequence of the gene upon deletion (Figure 9A). Successful knockout of FGFR1 gene was validated using western blot (WB) staining of FGFR1 protein in control and knockout cell line as well as DNA sequencing (Figures 9B and C). Park7 was used as a loading control in all western blotting experiments. The aforementioned protein was chosen over other conventional loading controls due to its high expression stability among different human tissues and cell lines (Wisniewski \& Mann, 2016). 
A

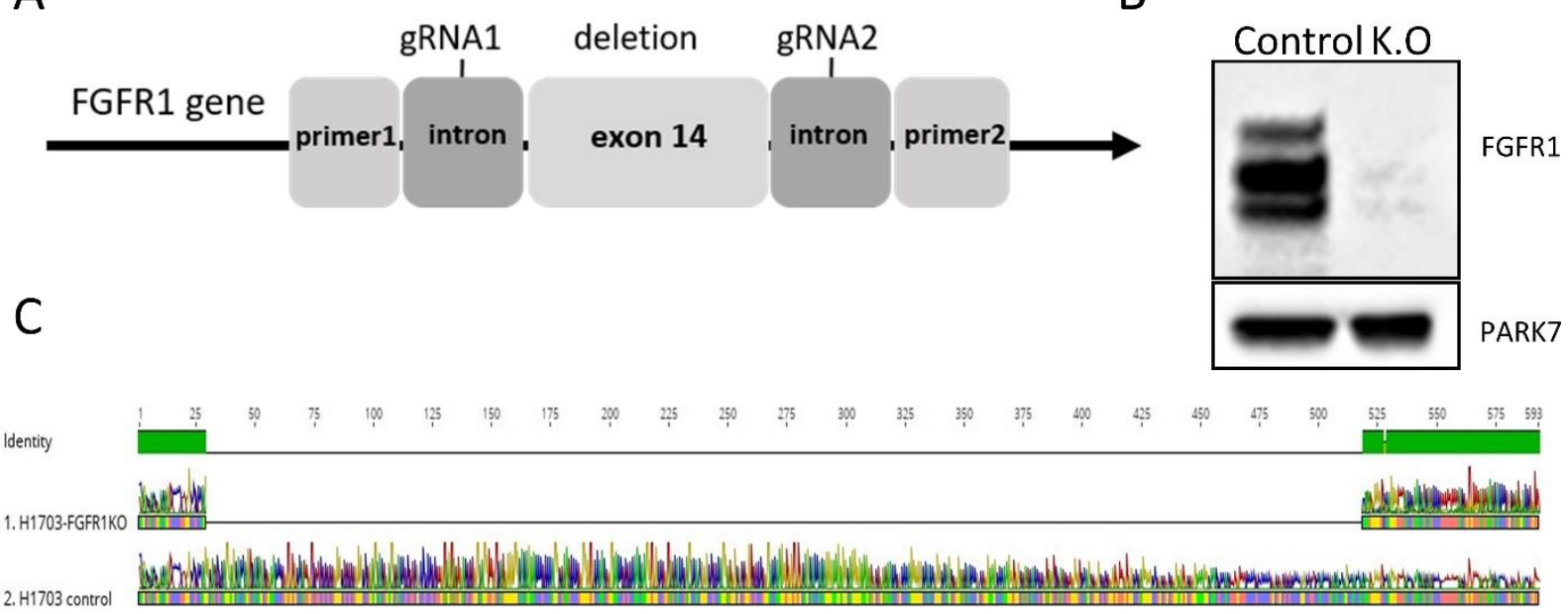

Figure 9: CRISPR/Cas9 knockout of FGFR1 gene in H1703 lung cancer cell line.

Diagram shows strategy of FGFR1 gene knockout using two different gRNAs (A). Western blot staining of FGFR1 protein expression in control $\mathrm{H} 1703$ cell line compared to FGFR1-knockout $\mathrm{H} 1703$ cell line (B). PARK7 was used as a loading control. DNA sequencing of exon 14 in $\mathrm{H} 1703$ control and knockout cell lines (C). Modified from: (Elakad et al., 2020).

Immunocytochemistry (ICC) staining of the knockout $\mathrm{H} 1703$ cell line showed a complete loss of FGFR1 signal compared to control H1703, which completed the validation of the anti-FGFR1 antibody as well as the protocol used for immunostaining (Figures 10A and B).

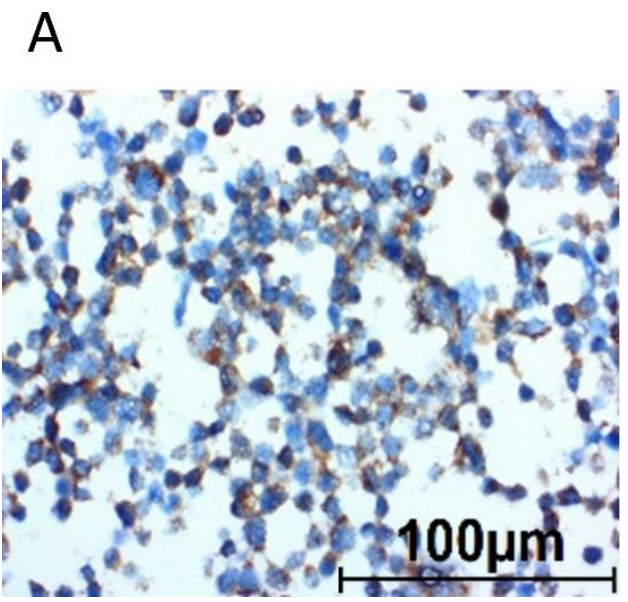

B

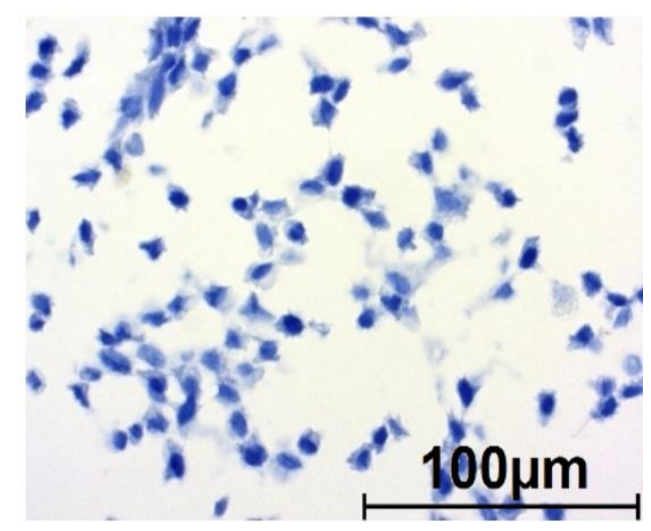

Figure 10: Validation of anti-FGFR1 antibody using the FGFR1-knockout cell line.

Immunocytochemistry staining of FGFR1 protein in control H1703 cell line (A) and FGFR1-knockout $\mathrm{H} 1703$ cell line (B). All images were captured at 40x magnification. Modified from: (Elakad et al., 2020). 


\subsubsection{FGFR1 protein expression in lung cancer}

FGFR1 protein expression levels were analyzed in SQCLC, AC and SCLC patient samples by immunohistochemistry $(\mathrm{IHC})$. Expression of FGFR1 protein was evaluated according to a three-level scoring system; strong expression, weak expression and no expression (Figures 11A-C).

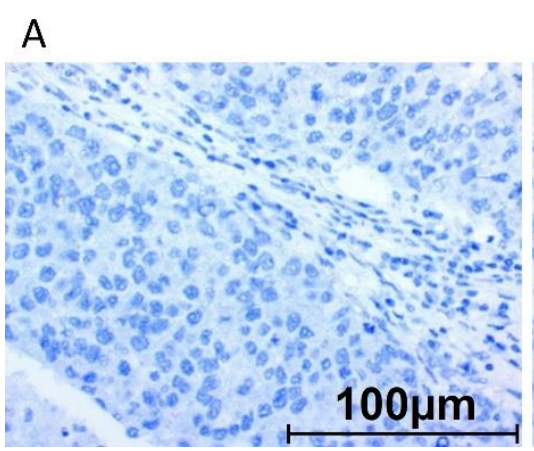

B

C

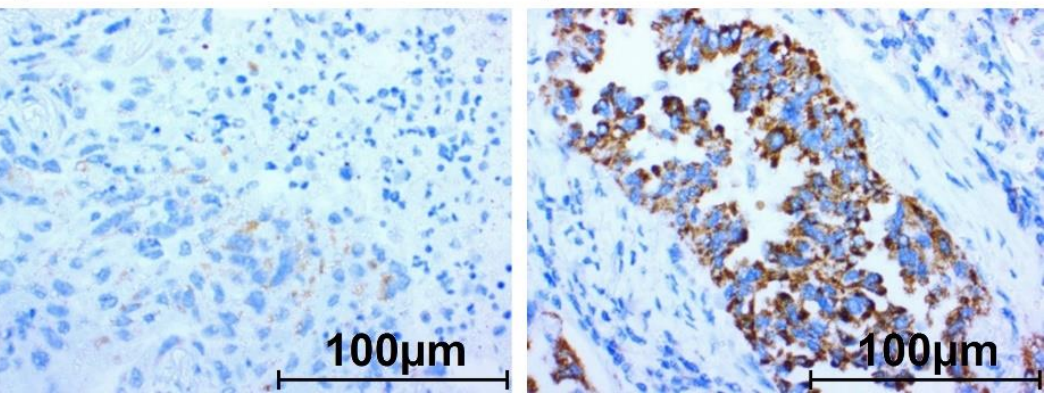

Figure 11: IHC staining of FGFR1 protein expression in SQCLC patient tissue samples.

Images show level of FGFR1 protein expression in negative sample (A), weak expression sample (B) and strong expression sample (C). All images were captured at 40x magnification. Modified from: (Elakad et al., 2020).

FGFR1 protein was strongly expressed in 16 out of $171(9 \%)$ evaluable SQCLC samples. In SCLC samples, strong expression was detected in 2 out of 44 (4\%) evaluable samples, while in AC strong expression was detected in 40 out of 144 (35\%) evaluable samples (Figure 12).

\section{FGFR1 protein expression prevalence}

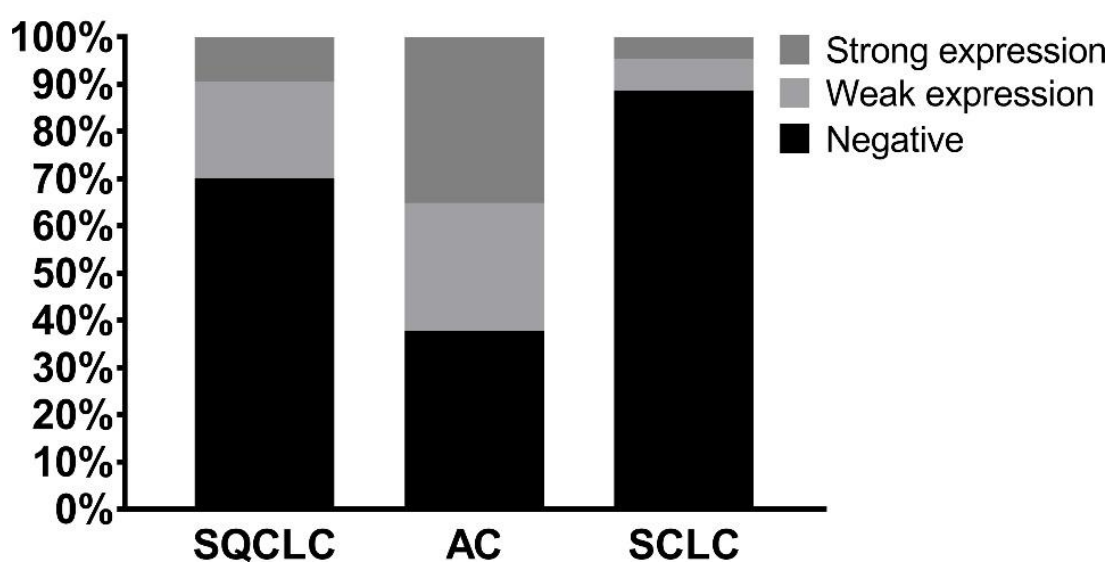

Figure 12: FGFR1 protein expression in SQCLC, SCLC and AC patient tissue samples.

Immunohistochemistry detected strength and prevalence of FGFR1 protein expression in SQCLC, SCLC and AC patients. Modified from: (Elakad et al., 2020). 
Next, I tested if FGFR1 protein expression can predict overall survival of patients through correlating patients' survival to expression levels in the three lung cancer groups. Kaplan-Meier curves alongside with chi-square analysis showed correlation $p$-values of $p=0.922, p=0.72$ and $p=0.44$ in SQCLC, SCLC and AC, respectively. Survival analysis showed that there was no significant correlation between expression level of FGFR1 and overall survival in any of the three tested groups (Figures 13A-C).

A

FGFR1 IHC SQCLC

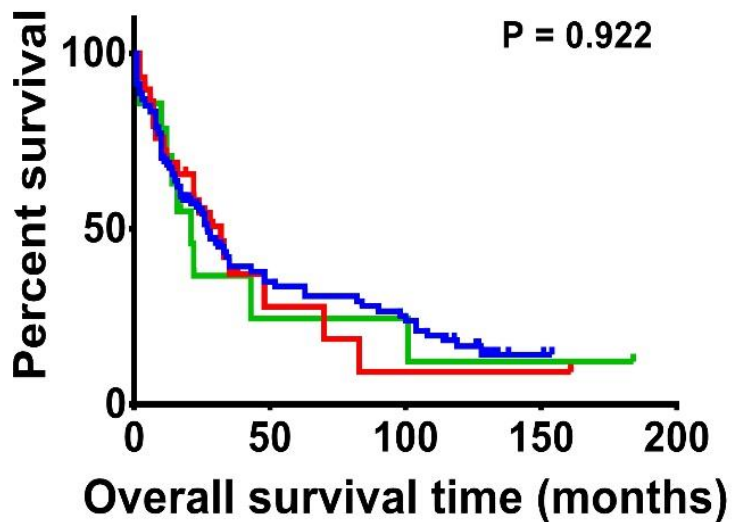

+ Negative

- Weak expression

+ Strong expression

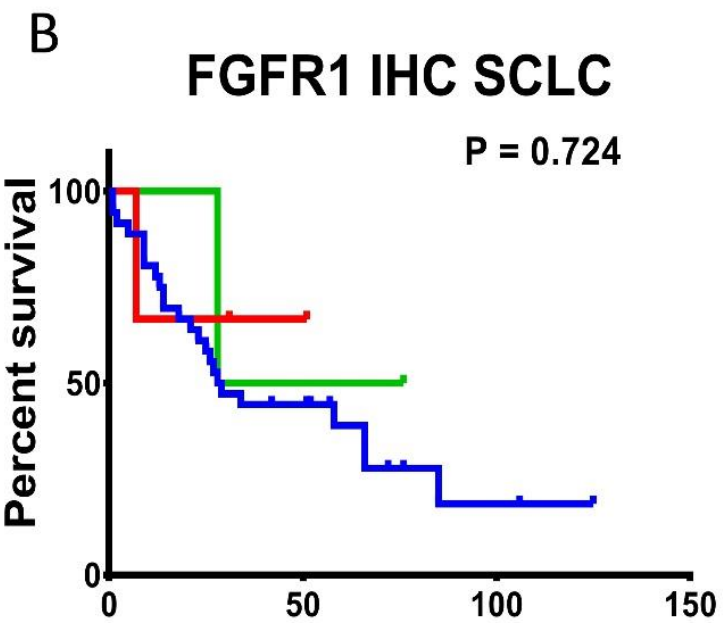

Overall survival time (months)
C

FGFR1 IHC AC

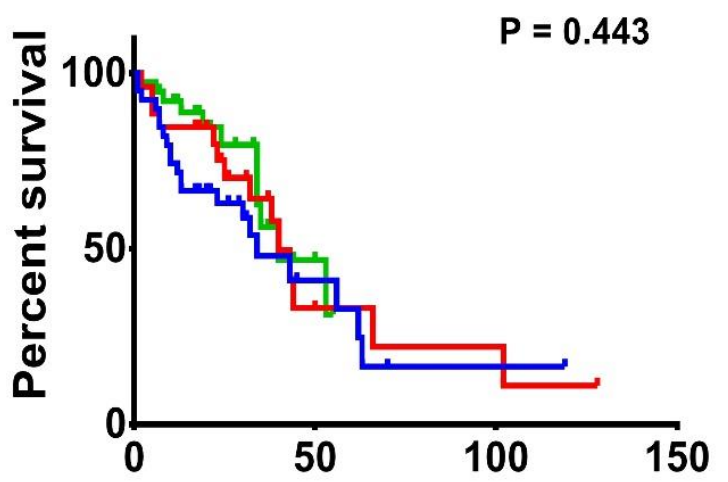

Overall survival time (months)

Figure 13: Correlation between FGFR1 protein expression and lung cancer patients' overall survival.

Kaplan-Meier analyses showed no significant association between FGFR1 expression and survival in SQCLC (A), SCLC (B) and AC (C). Statistical analysis was carried out using Chi-square test. Modified from: (Elakad et al., 2020). 


\subsubsection{Correlation between FGFR1 gene amplification and protein expression in lung cancer}

In order to check if FGFR1 gene amplification is constantly accompanied by protein overexpression, I correlated FISH to IHC data in SQCLC and SCLC groups. In SQCLC group, both amplification and protein expression together were evaluable in 129 samples. Correlation showed that in the amplified SQCLC group, 35\% were positive for protein expression, while $65 \%$ were negative. In the non-amplified SQCLC group, $29.6 \%$ were positive for FGFR1 expression, while $70.4 \%$ were negative. Chi-square test showed non-significant correlation $(p=0.54)$ between FGFR1 gene amplification tested by FISH and protein expression tested by IHC (Figure 14A). In SCLC group, 36 samples were evaluable for both FGFR1 gene amplification and protein expression from the same set. Only four samples showed FGFR1 protein expression with no gene amplification and three SCLC samples were positive for FISH and negative for IHC ( $p=0.99)$ (Figure 14B).

In the interest of expanding the correlation test between FGFR1 amplification and protein expression, I evaluated the correlation in 14 lung cancer cell lines. Gene amplification was tested through FISH analysis and protein expression was tested with western blot and ICC. In amplified FGFR1 cell lines, 4 out of $5(80 \%)$ cell lines showed strong expression of FGFR1 protein. In non-amplified FGFR1 cell lines, three out of nine $(33 \%)$ cell lines showed strong FGFR1 protein expression. Chi-square analysis showed a non-significant correlation between the two factors $(p=0.09)$ (Figure 14C). 
A

FISH vs. IHC in SQCLC

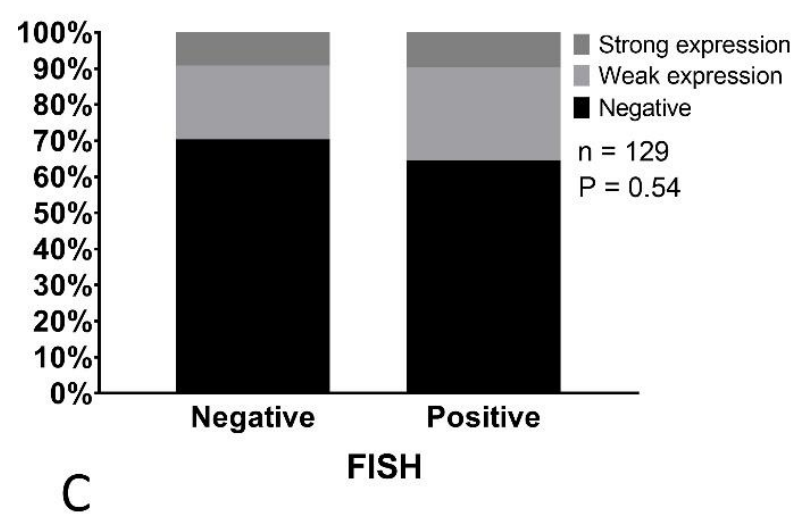

FISH vs. ICC/WB in lung cancer cell lines

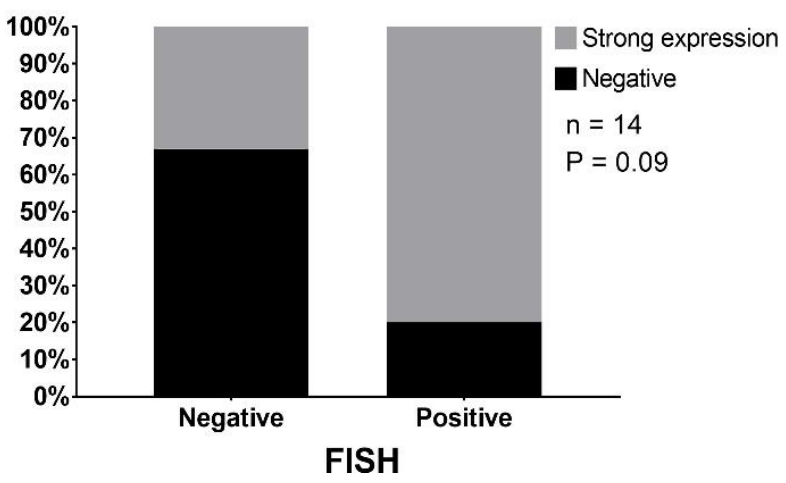

B

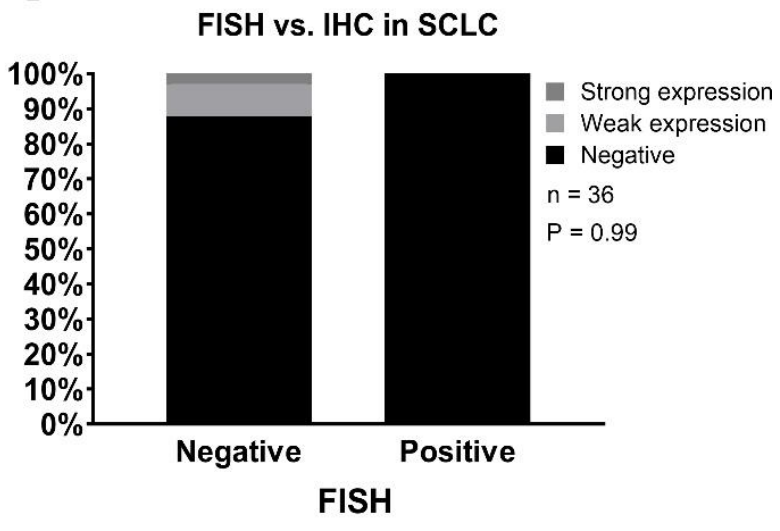

Figure 14: Correlation between FGFR1 gene amplification and protein expression.

FGFR1 gene amplification tested by FISH analysis was compared to FGFR1 protein expression tested by IHC in SQCLC patient tissues (A) and SCLC patient samples (B) or by ICC and western blot in lung cancer cell lines $(C)$. Statistical analysis was carried out using Chi-square test. Modified from: (Elakad et al., 2020). 


\subsection{Studying mechanisms of resistance to FGFR1 inhibition in lung cancer}

\subsubsection{Screening sensitivity to FGFR1 inhibition in human lung cancer cell lines}

Clinical trials of FGFR1 inhibitors have proven the concept of efficacy in FGFR1 amplified SQCLC patients. However, moderate partial response and progression free survival outcomes have suggested existence of resistance mechanisms that originally exist in patients or develop subsequent to inhibitors exposure.(Aggarwal et al., 2019; Nogova et al., 2017) In order to study mechanisms of resistance to FGFR1 inhibition, an appropriate cell line model had to be established. For this aim, I analyzed sensitivity to FGFR1 inhibition in 21 human lung cancer cell lines. DMS114, DMS53, H1339, H69, H82, HCC33, OH1, OH3, SCLC21H, SHP77 and SW2 are small cell lung cancer cell lines. H1703, H2170, HCC15, H520, H226, LK2, EBC1 and SKMES1 are squamous cell lung cancer cell lines. H1581 and LCLC103H are large cell lung cancer cell lines.

MTS viability assay was used to test the sensitivity to FGFR1 inhibition. Cell lines were incubated with increasing concentrations of AZD4547 for 72 hours. The assay showed that, among the 21 cell lines tested, the three cell lines H1581, LK2 and DMS114 had the highest sensitivity to the FGFR1 inhibitor AZD4547 with IC50 values of $0.03 \mu \mathrm{M}, 0.05 \mu \mathrm{M}$ and $0.06 \mu \mathrm{M}$, respectively. On the other hand, the majority of cell lines (18 cell lines, $86 \%$ ) showed very low sensitivity to the inhibitor with IC50s $>3 \mu \mathrm{M}$ (Figure 15). 


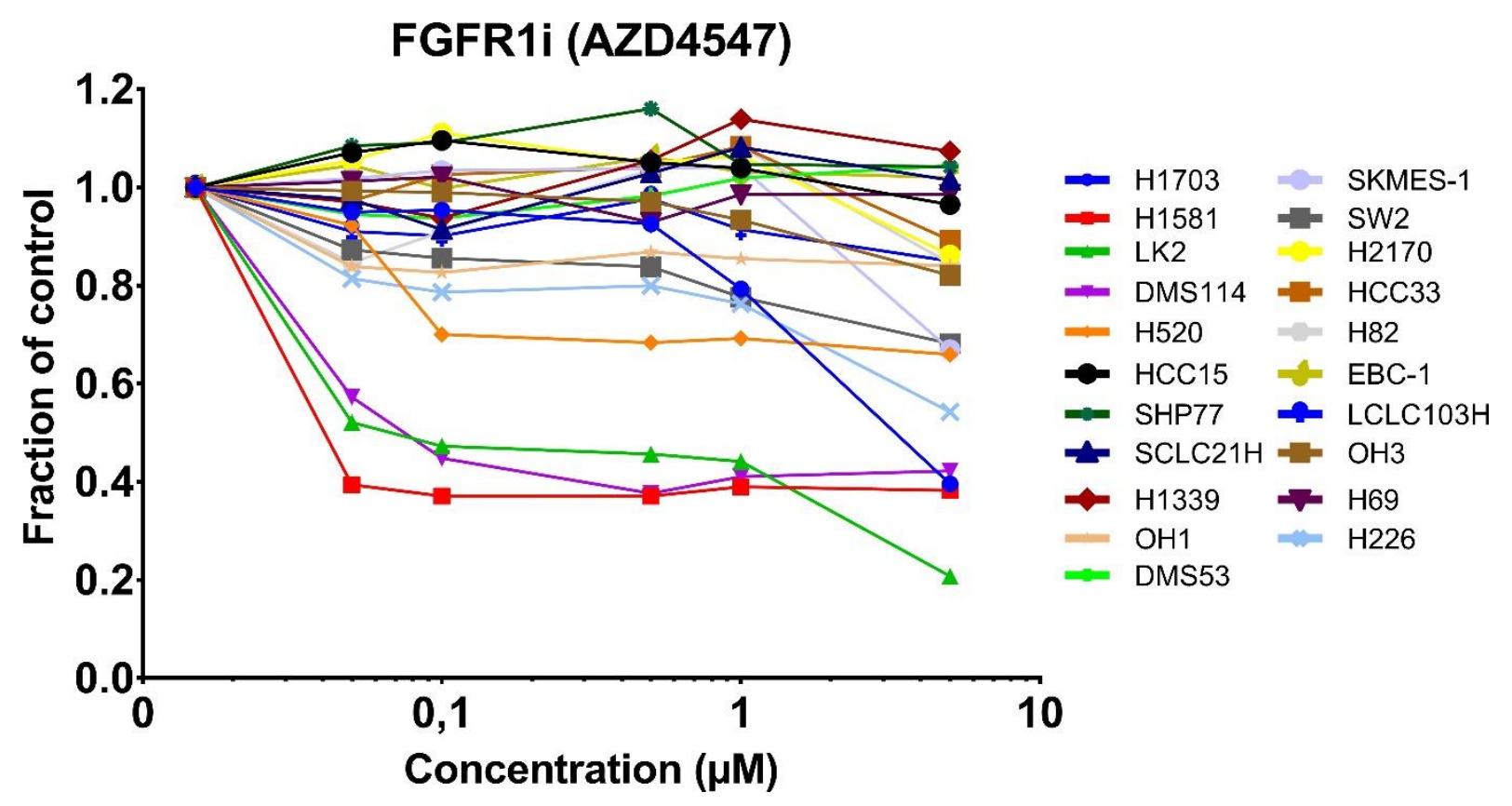

Figure 15: Screening sensitivity of lung cancer cell lines to FGFR1 inhibition.

Twenty-one human lung cancer cell lines were inhibited with the FGFR1 inhibitor AZD4547 at five increasing concentrations from 0.001 to $5 \mu \mathrm{M}$ for 72 hours. MTS assay showed viability of cell lines calculated as fraction of control cells treated with DMSO.

\subsubsection{FGFR1 amplification in human lung cancer cell lines}

Next, I tested FGFR1 gene amplification status in 11 of the previously described cell lines. Selection of the 11 cell lines was based on their sensitivity to the FGFR1 inhibitor AZD4547. FISH analysis technique was chosen to check for FGFR1 gene amplification in paraffin embedded cell blocks of the cell lines. Five cell lines (H1581, DMS114, H520, H1703 and DMS53) out of 11 cell lines tested (45.5\%) had an amplified FGFR1 gene copy number (Table 3). 
Table 3: FGFR1 gene amplification analyzed by fluorescence in situ hybridization (FISH) in multiple lung cancer cell lines.

\begin{tabular}{ccc}
\hline Order & Cell line & $\begin{array}{c}\text { FGFR1 gene } \\
\text { amplification (FISH) }\end{array}$ \\
\hline 1 & H520 & Amplified \\
2 & H1703 & Amplified \\
3 & DMS114 & Amplified \\
4 & H1581 & Amplified \\
5 & DMS53 & Amplified \\
6 & H226 & Non-amplified \\
7 & H2170 & Non-amplified \\
8 & HCC15 & Non-amplified \\
9 & LK2 & Non-amplified \\
10 & OH3 & Non-amplified \\
11 & SW2 & Non-amplified \\
\hline
\end{tabular}

\subsubsection{Assigning cell line model to study FGFR1 inhibition resistance in lung cancer}

With the purpose of building a representative cell line model, I included three groups of cell lines: a sensitive group, a resistant group and a control group. In the sensitive group, I chose the three cell lines H1581, LK2 and DMS114. H1581 and DMS114 cell lines showed FGFR1 gene amplification and high sensitivity to FGFR1 inhibition. LK2 showed no amplification of the FGFR1 gene but high sensitivity to FGFR1 inhibition. In the non-sensitive or resistant group, I chose the two cell lines $\mathrm{H} 520$ and $\mathrm{H} 1703$ with high FGFR1 amplification and expression level and low sensitivity to FGFR1 inhibition. In the control group, I chose HCC15 and H2170 cell lines with normal FGFR1 gene copy number and no sensitivity to FGFR1 inhibition (Figure 16). DMS53 cell line showed amplification of FGFR1 gene with no sensitivity to FGFR1 inhibition. However, DMS53 cell line showed minimal expression of FGFR1 protein, hence it did not fit any of the three groups (Figure 19). 


\section{Control group Resistant group Sensitive group}

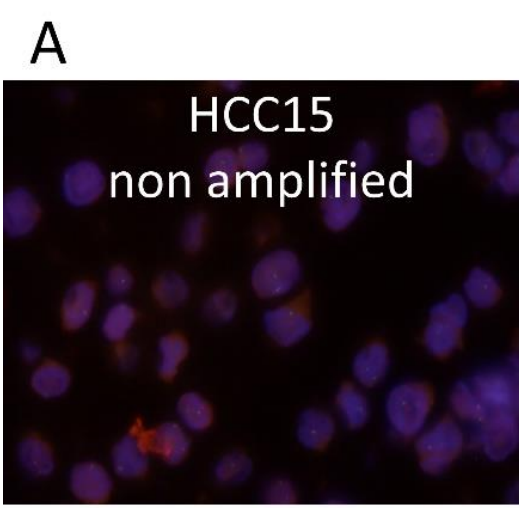

B

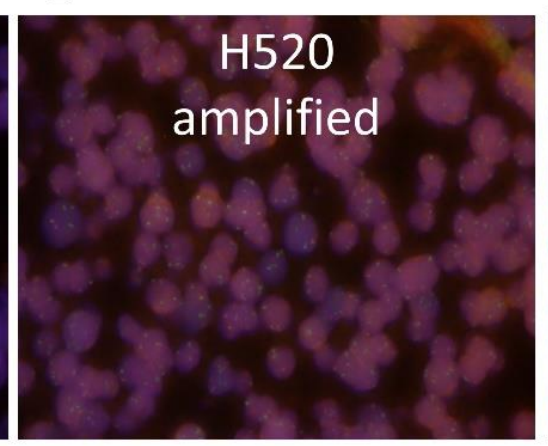

$\mathrm{E}$

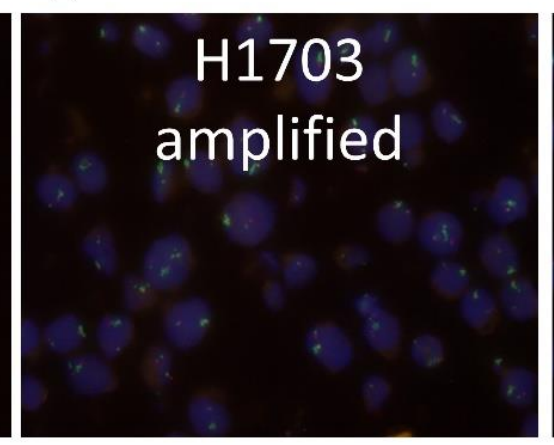

C

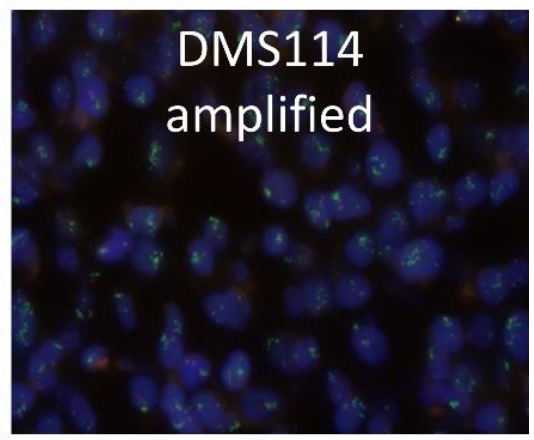

$\mathrm{F}$

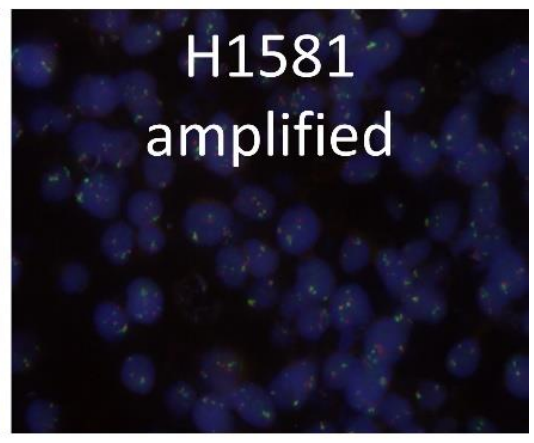

G

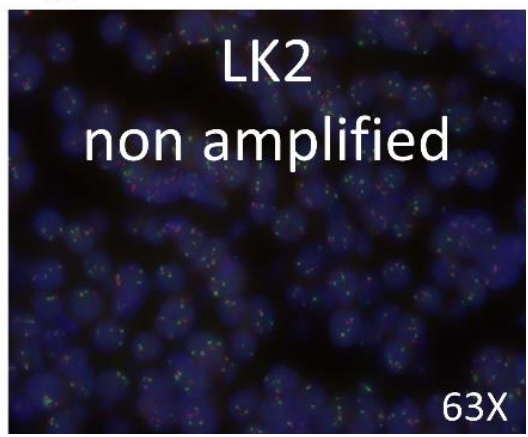

Figure 16: Prevalence of FGFR1 gene amplification (FISH analysis) in lung cancer cell lines.

FGFR1 gene amplification was detected by FISH analysis in control ( $A$ and $D)$, resistant $(B$ and $E)$ and sensitive ( $C, F$ and $G)$ cell lines to FGFR1 inhibition.

\subsubsection{FGFR1 protein expression in human lung cancer cell lines}

Next, I tested FGFR1 protein expression levels in the seven cell lines in my model. Protein expression levels were tested using western blotting (WB) and immunocytochemistry (ICC) using paraffin embedded cell blocks of the cell lines. Western blotting and ICC showed that FGFR1 protein was strongly expressed in five cell lines, which formed the sensitive and resistant groups (H1581, LK2, DMS114, 
H520 and H1703 cell lines). On the other hand, control group cell lines (HCC15 and H2170 cell lines) showed no FGFR1 protein expression (Figures 17 and 18).

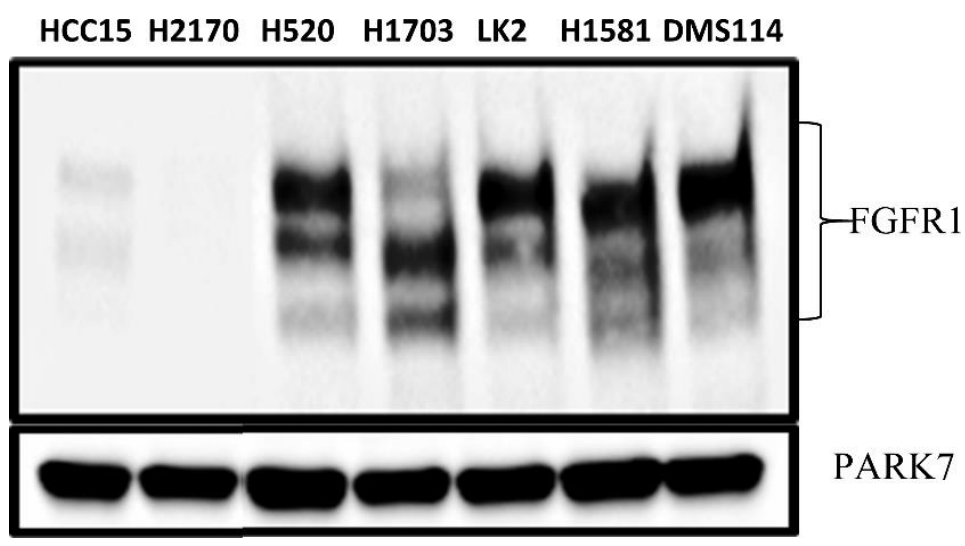

Figure 17. FGFR1 protein expression in multiple lung cancer cell lines.

Western blot showed FGFR1 expression in the seven lung cancer cell lines formed the model. PARK7 was used as a loading control. 


\section{Control group Resistant group Sensitive group}

A
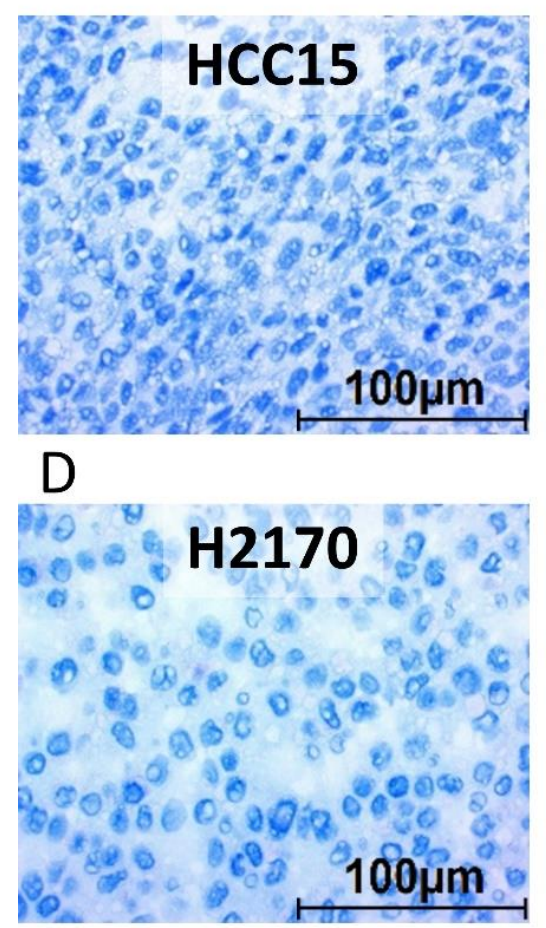

B

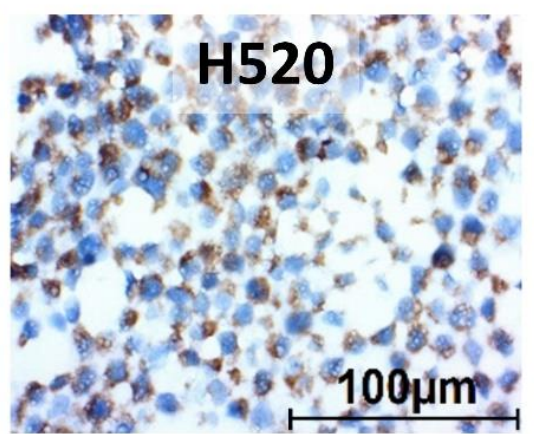

$\mathrm{E}$

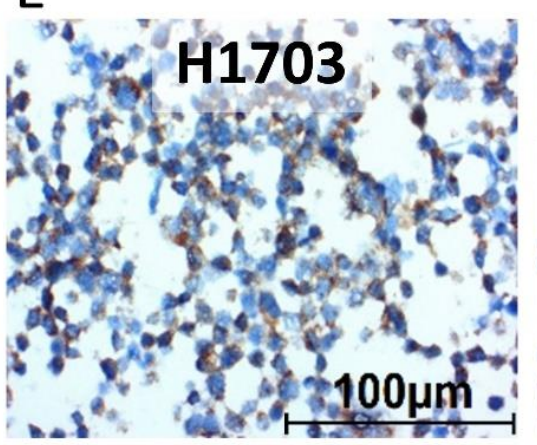

C

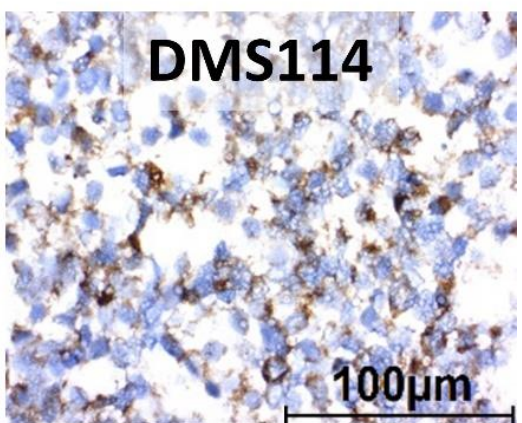

$\mathrm{F}$

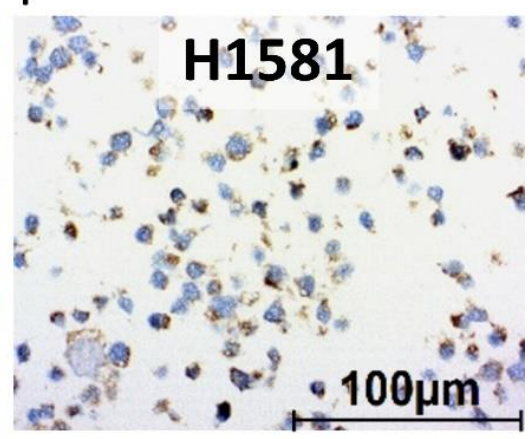

G

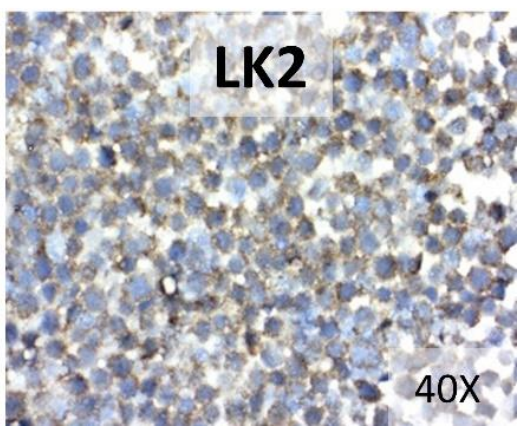

Figure 18: Immunocytochemical staining of FGFR1 protein in lung cancer cell lines.

ICC images showed levels of FGFR1 expression in control ( $A$ and $D$ ), resistant ( $B$ and $E$ ) and sensitive ( $C, F$ and $G$ ) cell lines to FGFR1 inhibition. All images were captured at $40 X$ magnification.

Collectively, my model consisted of three groups: a sensitive/responder group, a resistant group and a control group. The sensitive group included $\mathrm{H} 1581$ and DMS114 cell lines with FGFR1 gene amplification and strong protein expression and LK2 cell line with no FGFR1 gene amplification but with strong protein expression. The resistant group included $\mathrm{H} 520$ and $\mathrm{H} 1703$ cell lines with FGFR1 gene amplification and strong protein expression. Finally, the control group included HCC15 and H2170 cell lines with no FGFR1 gene amplification and negative protein expression (Figure 19). 


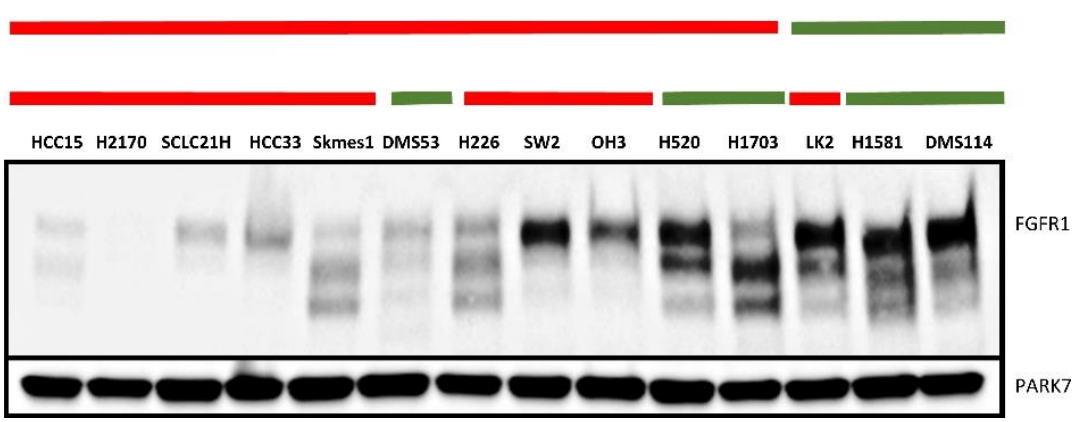

Figure 19: Collective status of FGFR1 gene amplification and protein expression in lung cancer cell lines.

Western blot showed expression of FGFR1 protein in 14 lung cancer cell lines. FGFR1 amplification was measured by FISH analysis. Sensitivity to the FGFR1 inhibitor AZD4547 was measured by MTS viability assay. FGFR1 protein expression was measured by western blot and immunocytochemistry. PARK7 was used as a loading control.

\subsubsection{Validation of sensitivity to FGFR1 inhibition in the cell line model}

The first step of validating sensitivity of the model to FGFR1 inhibition was done through MTS viability assay. The seven cell lines were incubated with increasing concentrations of AZD4547 (FGFR1 inhibitor) for 96 hours. The assay was repeated with three biological replicates and three technical replicates. MTS viability assay confirmed previous sensitivity pattern of the model where H1581, LK2 and DMS114 cell lines had IC50s $<0.1 \mu \mathrm{M}$, while H520, H1703, HCC15 and H2170 cell lines had IC50 > $5 \mu \mathrm{M}$. (Figure 20).

\section{FGFR1i (AZD4547) ( $\mu M$ M)}

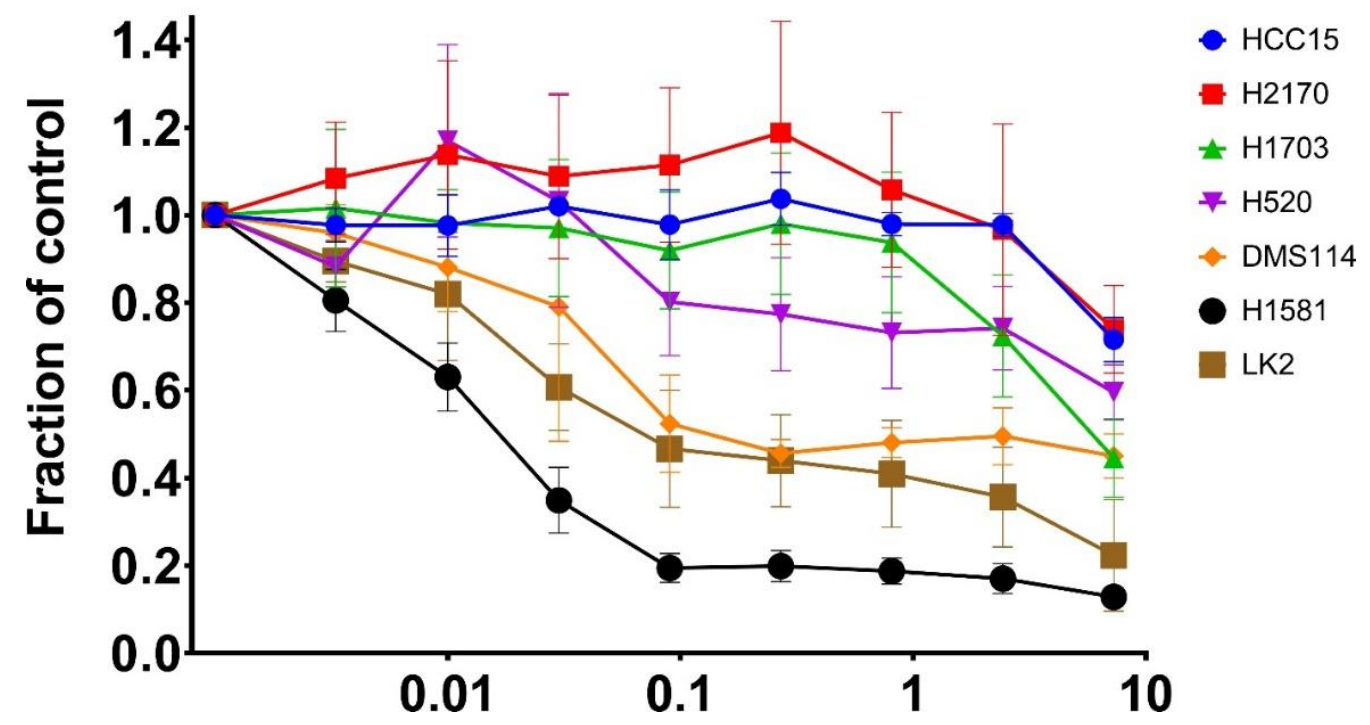


Figure 20: Sensitivity to the FGFR1 inhibitor AZD4547.

Sensitivity was measured via MTS viability assay after 96 hours incubation. Viability was calculated as fraction of control cells treated with the same concentration of DMSO. Nine increasing concentrations of AZD4547 were used $(0,0.003,0.01,0.03,0.1,0.27,0.8,2.4$ and $7.3 \mu \mathrm{M})$. Experiments were conducted in triplicates.

Next, I confirmed the previously detected response pattern of the cell lines through incubation with the FGFR1 inhibitor BGJ398 for 96 hours. H1581, DMS114 and LK2 cell lines showed IC50 values of $0.08,0.8$ and 1.2, respectively. H520, H1703, HCC15 and H2170 cell lines showed IC50 values higher than $5 \mu \mathrm{M}$ (Figure 21).

\section{FGFR1i (BGJ398) ( $\mu$ M)}

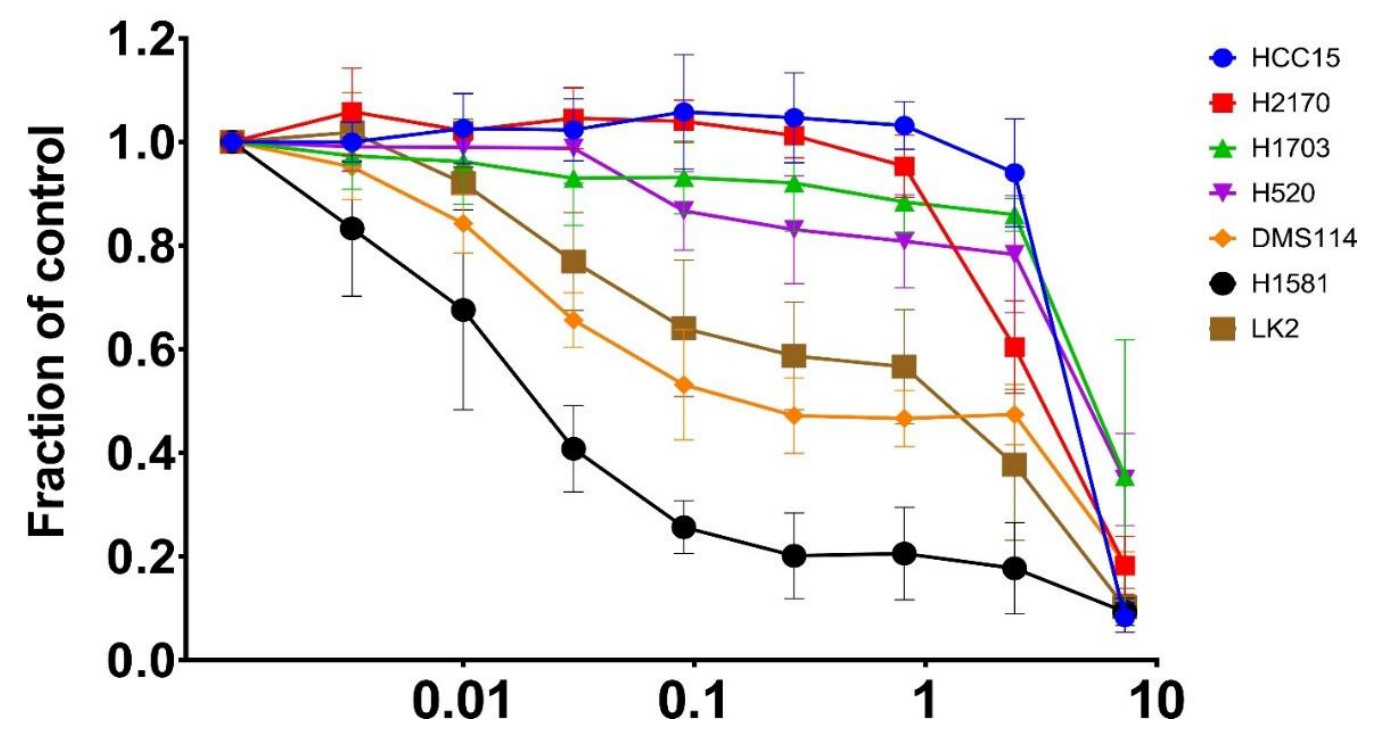

Figure 21: Sensitivity to the FGFR1 inhibitor BGJ398.

Sensitivity to the FGFR1 inhibitor BGJ398 measured through MTS assay after 96 hours incubation. Viability was calculated as fraction of control cells treated with the same concentration of DMSO. Nine concentrations of BGJ398 were used $(0,0.003,0.01,0.03,0.1,0.27,0.8,2.4$ and $7.3 \mu \mathrm{M})$. Experiments were conducted in triplicates.

Further validation of response to FGFR1 inhibition was achieved through counting number of viable cells (via Muse flow cytometry) after incubation with the FGFR1 inhibitor AZD4547 for 96 hours. Cell growth was evaluated as percentage of cells grown under inhibition compared to cells grown under vehicle (DMSO). The cell line H1581 showed the highest sensitivity to the inhibition with less than $17 \%$ viable cells 
compared to control. DMS114 and LK2 cell lines represented the second and third sensitive cell lines with growth percentages of $32 \%$ and $40 \%$, respectively. H520, $\mathrm{H} 1703$, HCC15 and H2170 cell lines showed non-significant reduction in growth compared to controls (Figure 22).

\section{Cell growth (Number of viable cells)}

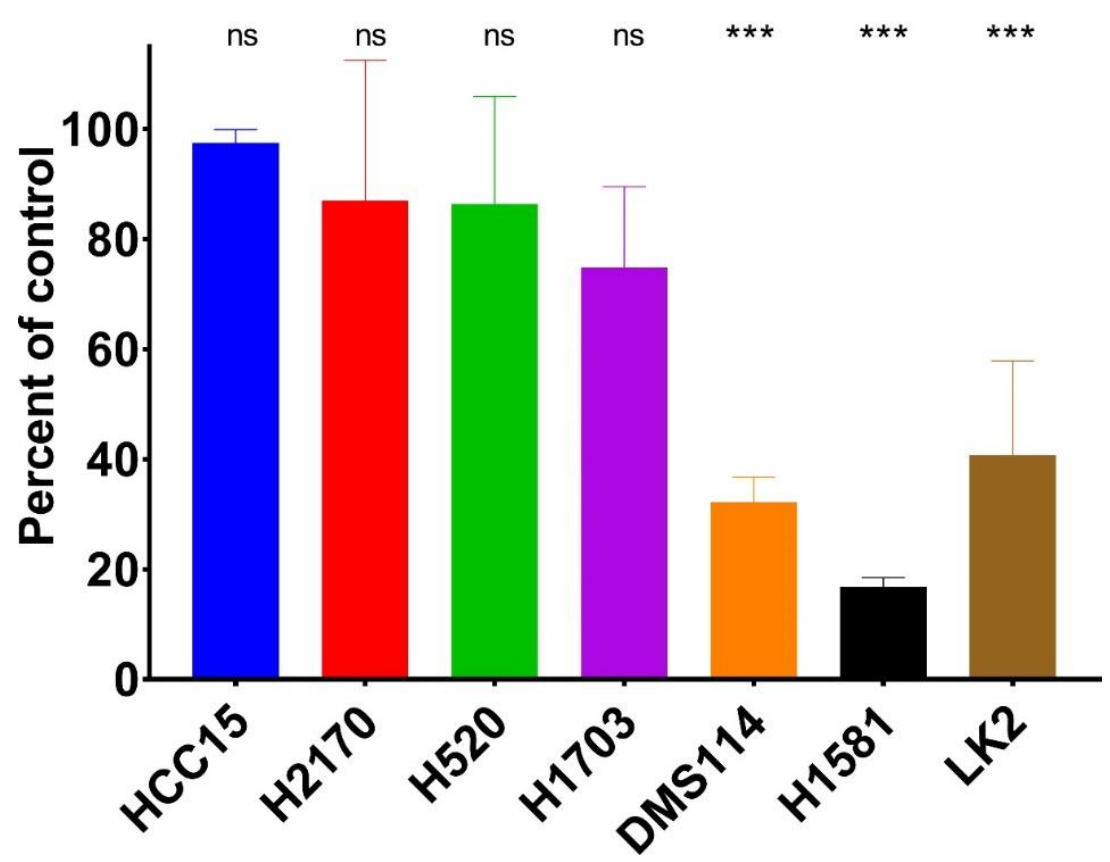

Figure 22: Cell growth assay (number of viable cells) after treatment with AZD4547.

Cell lines were incubated with $0.5 \mu \mathrm{M}$ of the FGFR1 inhibitor AZD4547 for 96 hours. Absolute numbers of viable cells were counted using Muse cell analyzer. Experiments were conducted in triplicates. $P$ values were denoted as $n s=p>0.05$ and ${ }^{* \star *}=p \leq 0.001$.

\subsubsection{Testing AZD4547 (FGFR1 inhibitor) phosphorylation inhibition function}

AZD4547 is a specific tyrosine kinase inhibitor, which suppresses activation/phosphorylation of FGFR1 at its tyrosine residues through ATP competition. To test the efficiency of AZD4547 in inhibiting FGFR1 phosphorylation, I incubated cell lines with $0,5 \mu \mathrm{M}$ AZD4547 for 3 hours. Immunoblotting showed a significant decline in phospho-FGFR1 signals at Y654 site upon incubation with the inhibitor compared to incubation with DMSO in H1703, H520, DMS114, H1581 and LK2 cell lines. HCC15 and H2170 control cell lines lacked original phosphorylation signal of FGFR1 under control conditions due to minimal expression of FGFR1 protein in these cell lines (Figure 23). 


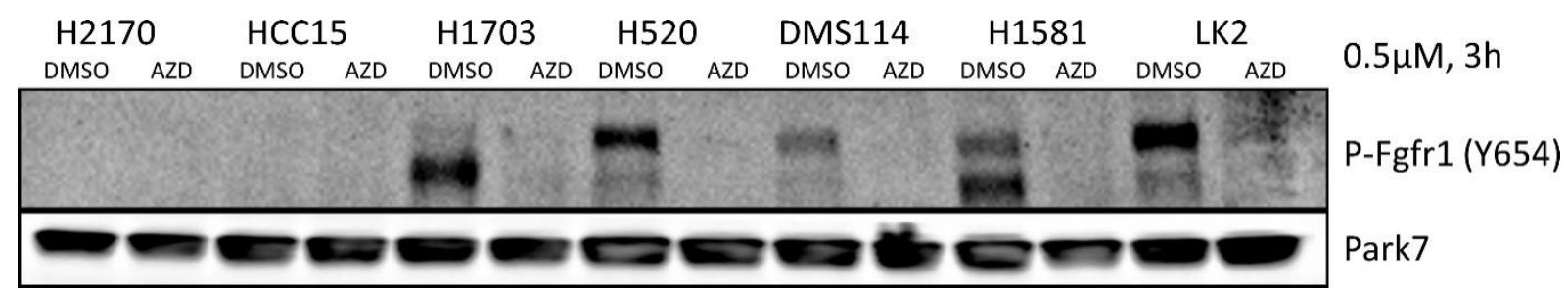

Figure 23: Phosphorylation inhibition through AZD4547.

Western blot showed activity of AZD4547 in blocking phosphorylation at FGFR1 receptor. Cell lines were incubated with $0.5 \mu \mathrm{M}$ of AZD4547 for 3 hours. PARK7 was used as a loading control.

\subsubsection{Testing AZD4547 (FGFR1 inhibitor) specificity using siRNA}

To exclude off-target inhibition as a reason for response to FGFR1 inhibition, I knocked down FGFR1 gene via small interfering RNA (siRNA). Two different sequences of siRNA, which were validated by the manufacturer, were used to knock down FGFR1 in the seven cell lines. A scrambled siRNA, that does not target any sequence in human genome, was used as a control (Figure 24).

The first step in knocking down the FGFR1 gene was to test transfection efficiency of the transfection reagent (HiPerFect) and siRNAs in the described cell lines. Cell lines were incubated for 24 hours with a control siRNA coupled with a fluorescent dye. Control cells were incubated only with the transfection reagent. Percentages of fluorescent cells were detected using FACS cell analyzer. Transfection efficiency was higher than $90 \%$ in all cell lines except $\mathrm{H} 2170$ cell line with an efficiency of $82 \%$. Viability of cell lines was higher than $90 \%$ in all cell lines compared to controls. 


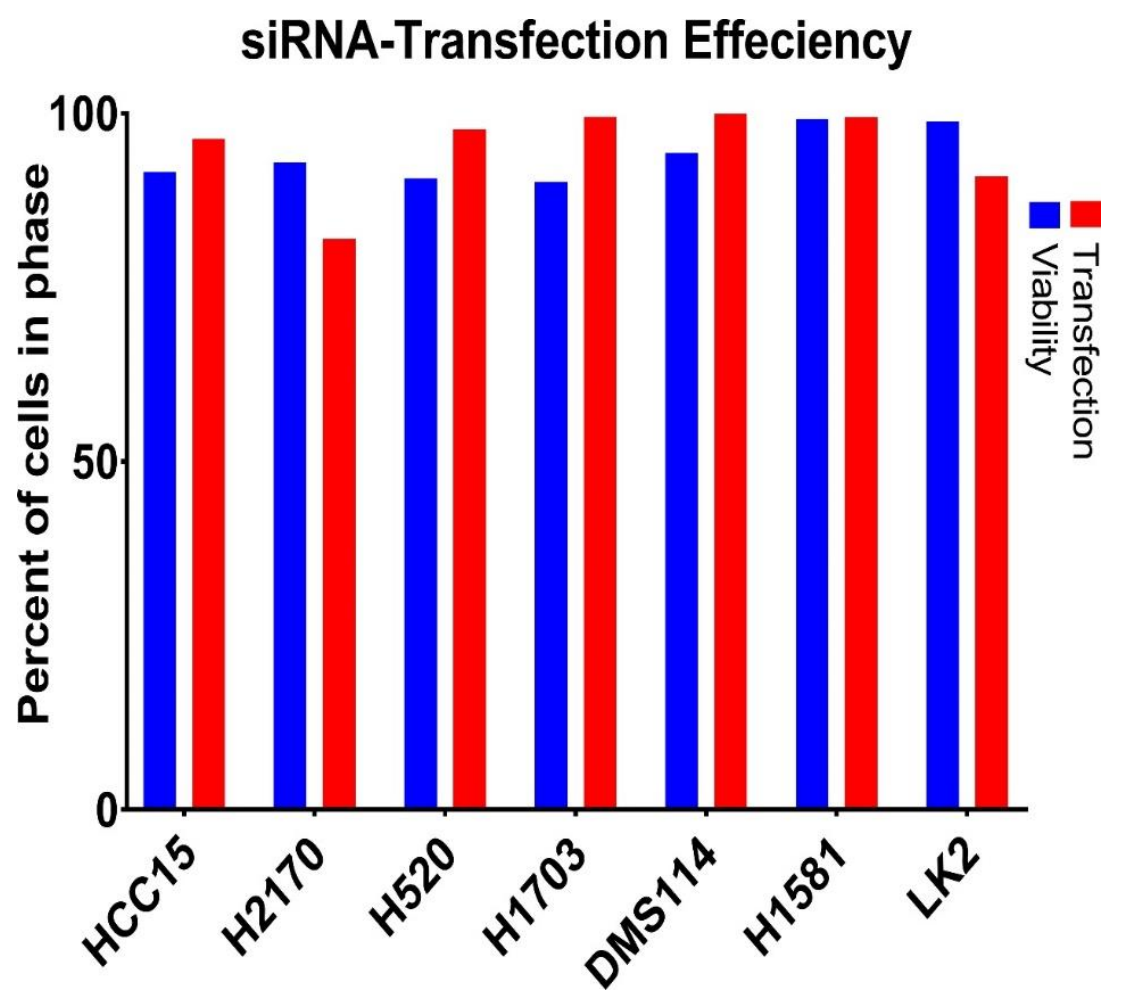

Figure 24: Transfection efficiency of siRNAs in lung cancer cell lines.

Transfection efficiency of siRNA was measured through FACS analyzer after 24 hours incubation with control siRNAs coupled with a fluorescent dye (Alexa Fluor 488). Viability of cell lines was measured through Propidium iodide staining.

Next, I transfected cell lines with $30 \mathrm{nM}$ of siRNA targeting FGFR1 and tested the efficiency of FGFR1 knockdown after 48 hours. H1581, DMS114, H520 and H1703 cell lines showed strong reduction in FGFR1 expression, LK2 cell line showed intermediate reduction and the control cell lines HCC15 and $\mathrm{H} 2170$ lacked original expression of FGFR1 under control conditions (Figure 25).

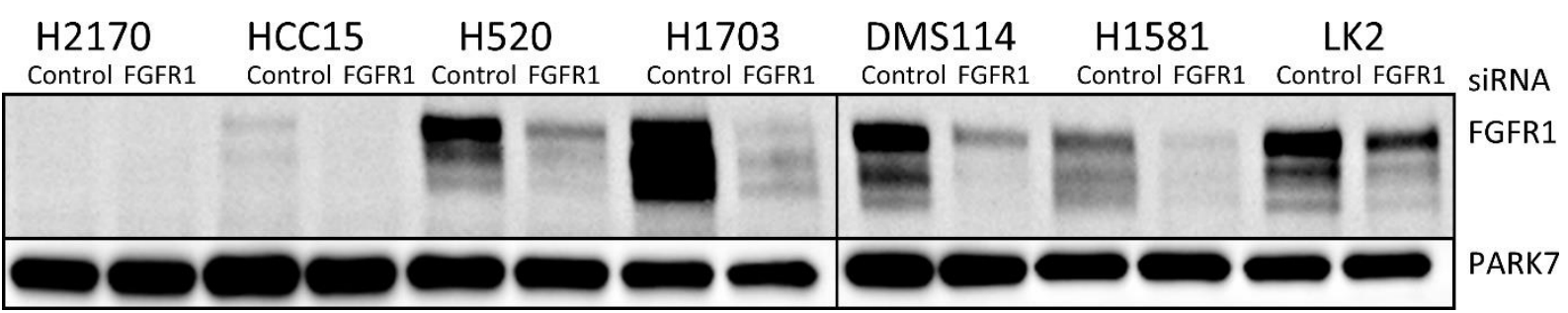

Figure 25: siRNA knockdown of FGFR1 protein.

Cell lines were transfected with $30 \mathrm{nM}$ of FGFR1-siRNA for 72 hours. PARK7 was used as a loading control. 
Finally, to test dependency of the seven cell lines on FGFR1 signaling, cell lines were incubated with $30 \mathrm{nM}$ of either siRNA targeting FGFR1 or scrambled siRNA for six days. Proliferation of cell lines was measured every 24 hours through MTS viability assay. Proliferation was calculated as fraction of control at 24 hours incubation. The experiment was repeated with four biological replicates. HCC15, H2170, H520 and H1703 cell lines showed similar proliferation patterns between FGFR1-siRNA and control-siRNA groups without significant difference. H1581, LK2 and DMS114 cell lines showed reduction in proliferation upon incubation with FGFR1-siRNA compared to control-siRNA. Reduction in proliferation reached significance on the fifth day in H1581 and DMS114 cell lines and on the sixth day in LK2 cell line (Figure 26).

\section{Control group}
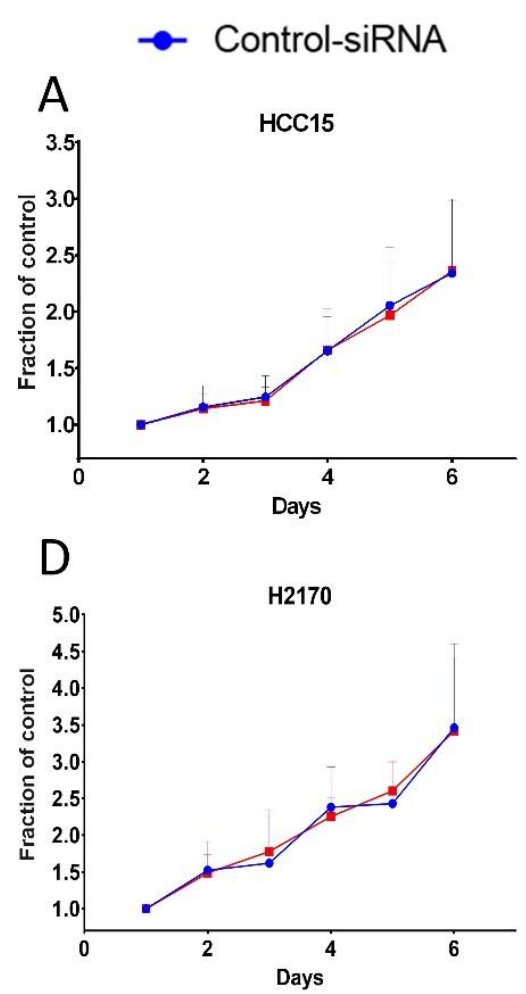

Resistant group

FGFR1-siRNA

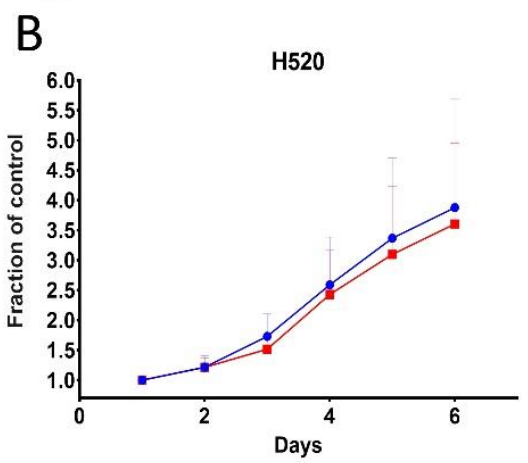

E

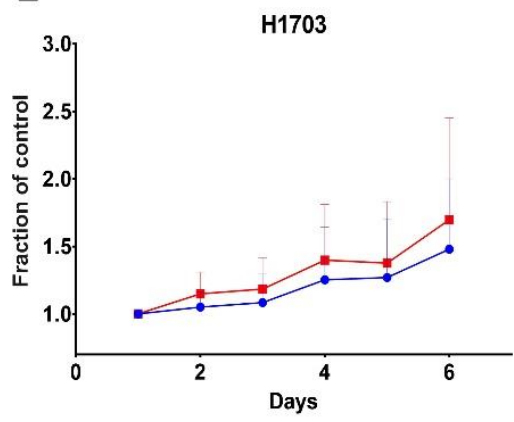

Sensitive group

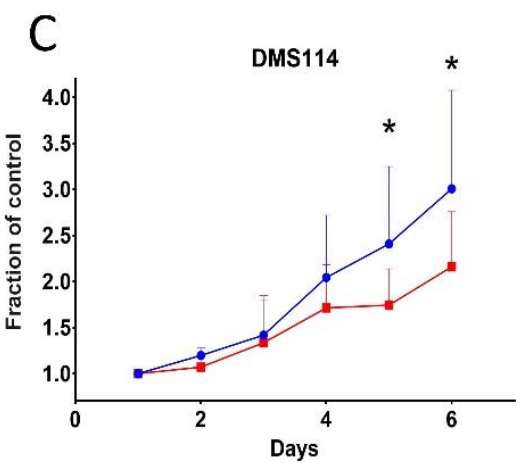

$\mathrm{F}$
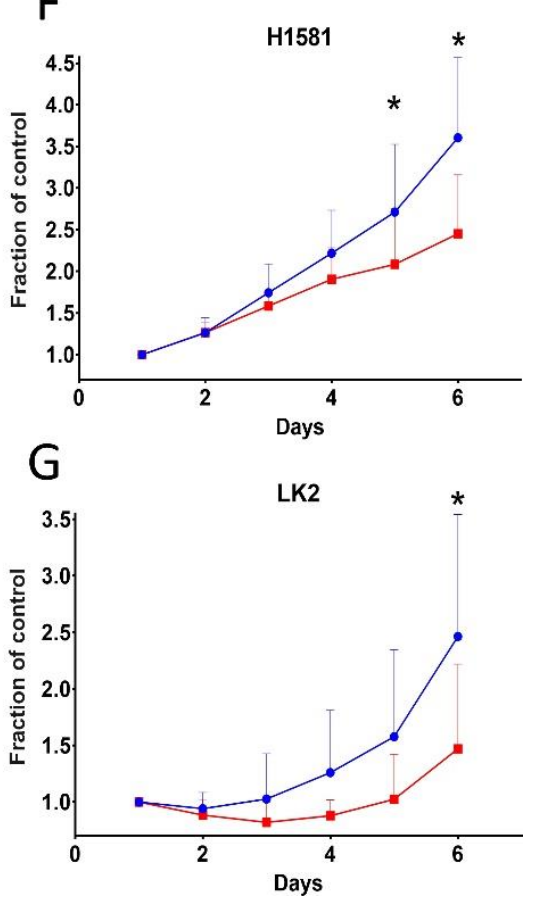
Figure 26: Proliferation assay under FGFR1-siRNA knockdown.

Cell lines were incubated with 30 nM of FGFR1-siRNAs for 6 days. Viability was measured every 24 hours using MTS viability assay. Experiments were conducted in quadruplicates. $P$ values were denoted as $n s=p>0.05$ and $^{*}=p \leq 0.05$.

\subsubsection{Exploring activated FGFR1 signaling in the cell lines model}

In order to study mechanisms of resistance to FGFR1 inhibition, I compared activated signaling pathways between the three groups of cell lines (control, resistant and sensitive groups). The seven cell lines in my model were incubated with either DMSO or the FGFR1 inhibitor AZD4547 at concentration of $0.5 \mu \mathrm{M}$ for 3 hours. Proteins were blotted on membranes and incubated with antibodies against the main effectors in FGFR1 signaling.

\begin{tabular}{|c|c|c|c|c|c|}
\hline HCC15 & H520 & H1703 & DMS114 & H1581 & LK2 \\
\hline$-\quad+$ & $-\quad+$ & + & $-\quad+$ & $-\quad+$ & $-\quad+$ \\
\hline
\end{tabular}
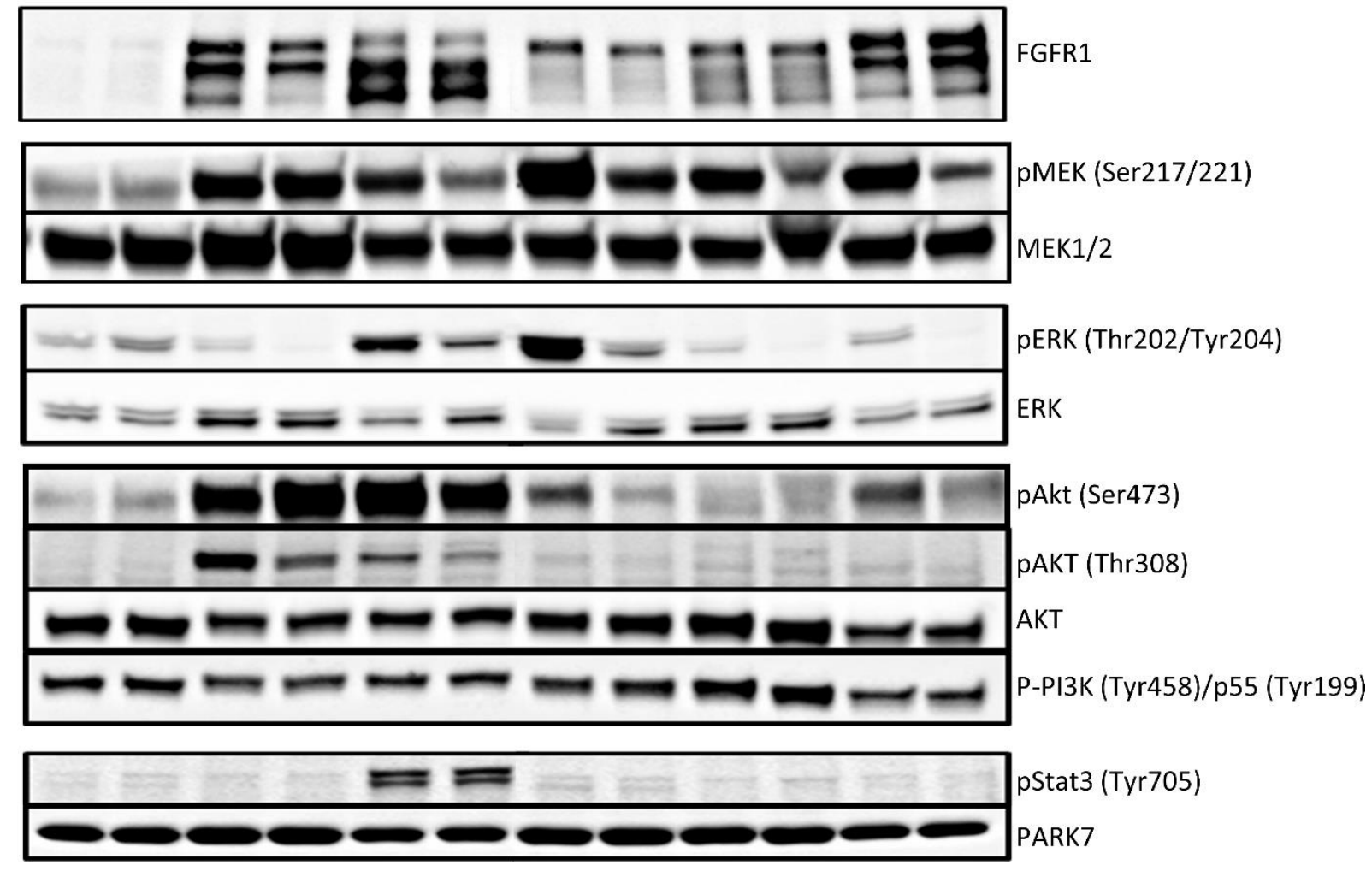

Figure 27: Western blot analysis of FGFR1 signaling in control, resistant and sensitive cell lines to FGFR1 inhibition.

Cell lines were incubated with either DMSO or the FGFR1 inhibitor AZD4547 for three hours. PARK7 was used as a loading control. 
Western blot analysis showed reduction in phosphorylated MEK (pMEK) signal in H1703 resistant cell line and DMS114, H1581 and LK2 sensitive cell lines upon inhibition by AZD4547. The control unphosphorylated MEK signal was unchanged. Activated ERK (pERK) signal was reduced in H520, H1703, DMS114, H1581 and LK2 cell lines upon inhibition by AZD4547 compared to DMSO.

Phosphorylated Akt signal, particularly at the serine residue (Ser473), represented the major difference in signaling between the three groups (control, resistant and sensitive). The resistant cell lines $\mathrm{H} 520$ and $\mathrm{H} 1703$ showed highly activated Akt signals (Ser473 and Thr308) compared to the sensitive cell lines or the control cell line. Phosphorylated signals were reduced upon incubation with AZD4547. Basal Akt signal was unaltered between control and inhibition conditions of each cell line or between the three groups of cell lines. Activated PI3K signal was comparable between the two conditions in each cell line and between the three groups of cell lines. Stat3 was not activated in any of the cell lines except of H1703. PARK7 was used as a loading control (Figure 27).

In order to expand the comparison between protein expression levels in the model, I used Archer Fusion directed thyroid and lung cancer next generation sequencing (CTL) panel. The panel enabled me to detect RNA levels among the different cell lines (Figure 28). RNA sequencing heat map showed high elevation of FGFR1 expression in the resistant cells ( $\mathrm{H} 520$ and $\mathrm{H} 1703$ ) as well as in the sensitive cells (DMS114, H1581 and LK2). FGFR1 levels were very low in the control cells (HCC15 and $\mathrm{H} 2170$ ) compared to other cell lines. The panel showed elevation of Akt1 signal in $\mathrm{H} 520$ cell line, while only slight elevation of the same signal was detected in $\mathrm{H} 1703$ cell line compared to other cell lines. No other significant differences were shown between the three groups of cell lines. 


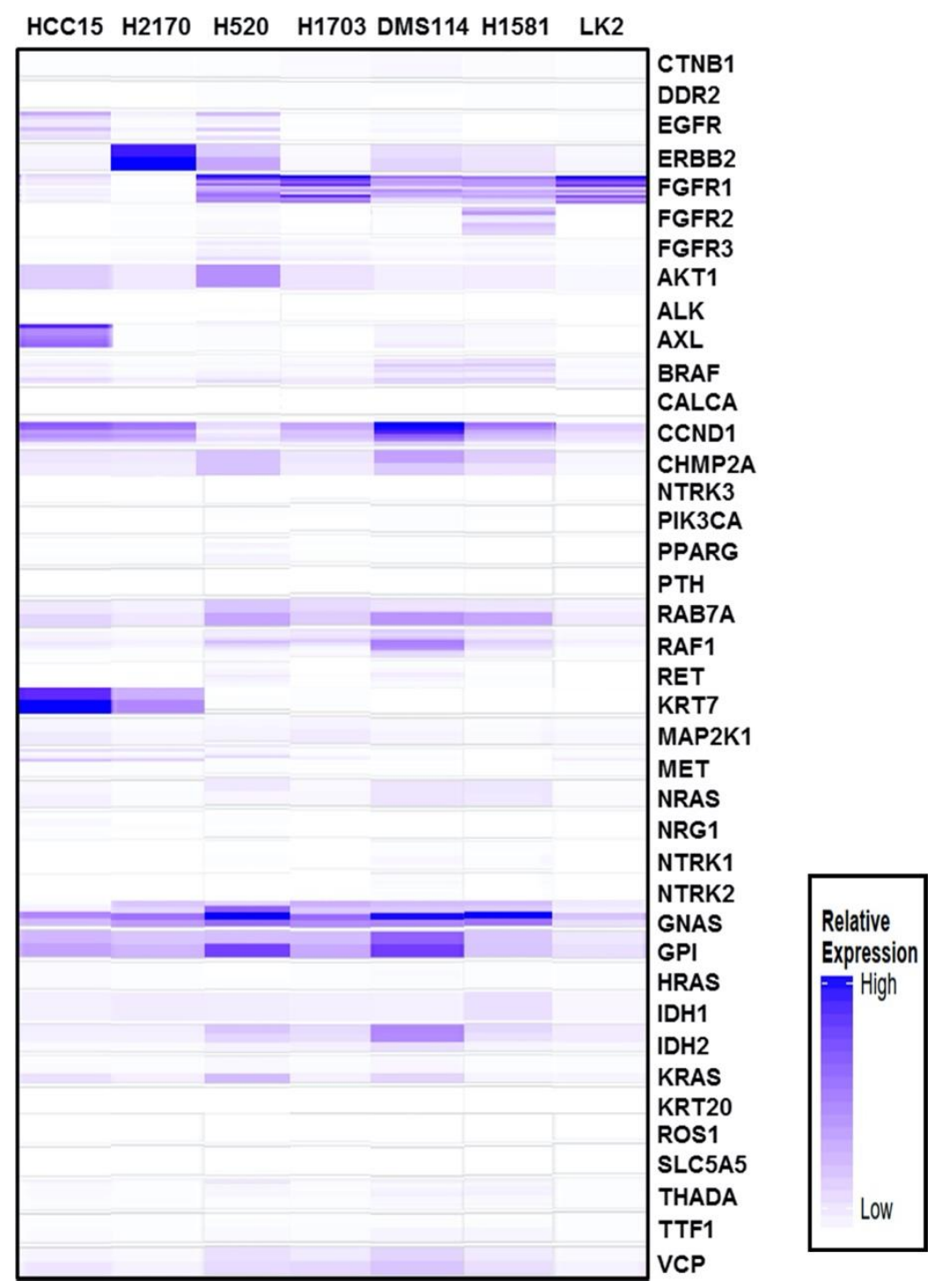

Figure 28: RNA-sequencing using Archer FusionPlex CTL Panel.

Next generation sequencing directed panel compared RNA expression levels among different control, resistant and sensitive cell lines.

\subsubsection{Activation of Akt in FGFR1-amplified SQCLC tissue samples}

Western blot analysis showed that overactivation of Akt was a major distinction between FGFR1 signaling in FGFR1 inhibition resistant and sensitive cell lines. Therefore, I tested if variation of Akt activation takes place in human FGFR1amplified and FGFR1-expressing SQCLC patient samples. I stained 32 FGFR1amplified and 45 FGFR1-expressing SQCLC patient samples against phosphorylated Akt using IHC. Staining was analyzed under microscope and phosphorylation levels of Akt were scored on a scale from 0 to 300 (H-score). Diagnosis of phospho-Akt 
expression in lung cancer patient tissues was done in collaboration with Sha Yao at the institute of Pathology, University Medical Center of Goettingen, Germany.

In FGFR1-amplified samples, 4 out of 32 samples (12.5\%) lacked Akt phosphorylation completely. Intermediate/weak phosphorylation was measured in 22 out of 32 samples (68.7\%), while strong phosphorylation of Akt (H-score > 240) was detected in 6 out of 32 FGFR1-amplified samples (18.8\%). In FGFR1-expressing samples, $15.5 \%, 55.6 \%$ and $28.9 \%$ showed negative, weak and strong Akt activation signals, respectively (Figures 29-30).

A

Negative Akt phosphorylation

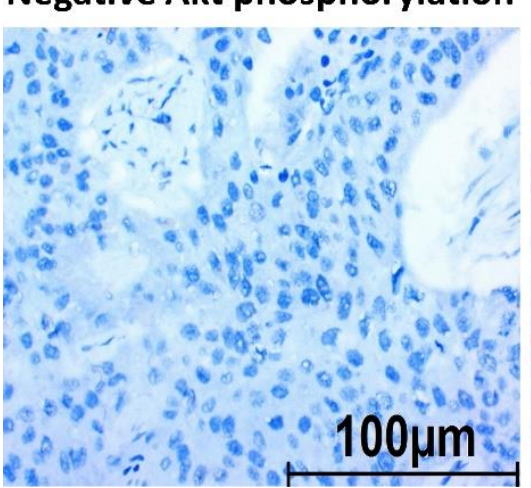

B

Weak Akt phosphorylation
C

Strong Akt phosphorylation
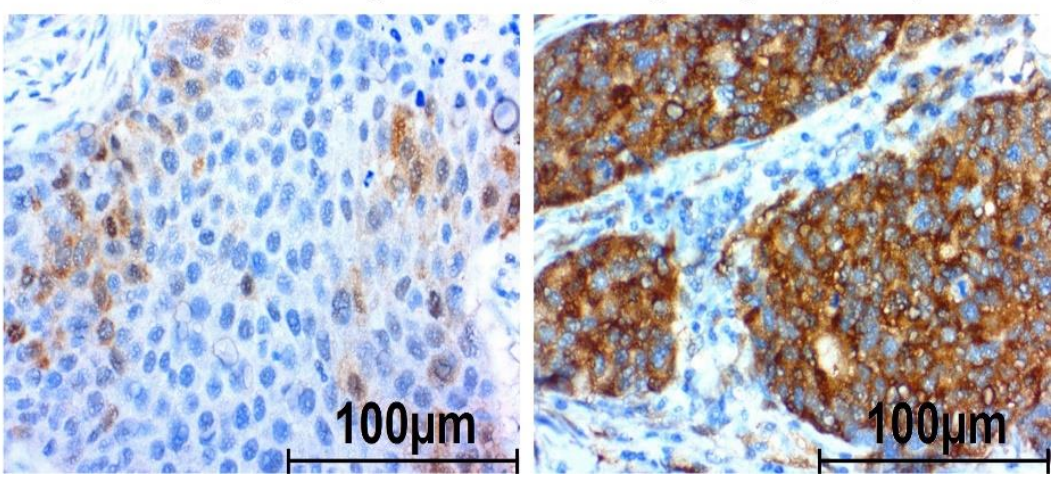

Figure 29: Immunohistochemical staining of pAkt in patient samples.

Images show examples of negative (A), Weak (B) and strong (C) phosphorylation of Akt in FGFR1amplified SQCLC patient samples. All images were captured at 40-x magnification.

A

Phosphorylation of Akt in FGFR1-amplified SQCLC patient samples

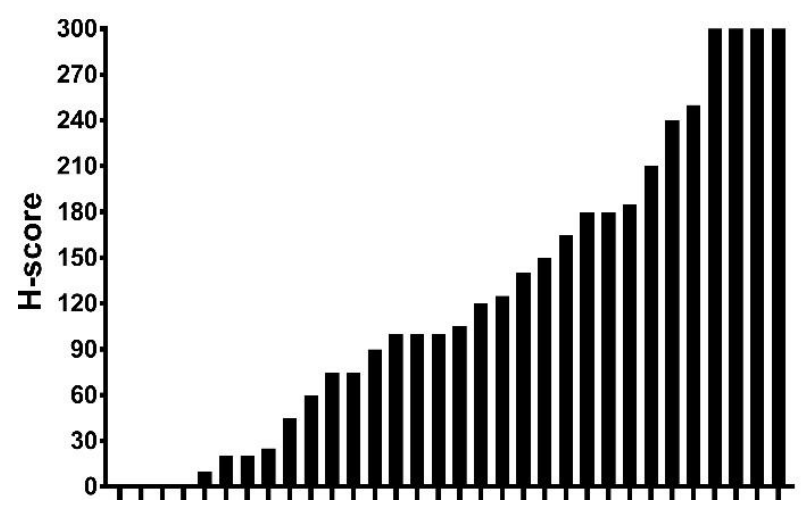

B

Phosphorylation of Akt in FGFR1-expressing SQCLC patient samples

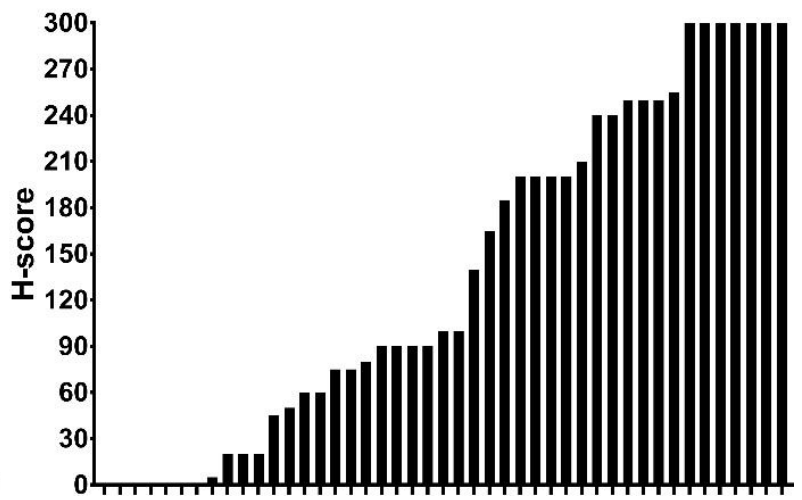

Figure 30: Expression levels of phosphorylated Akt in FGFR1-amplified and FGFR1-expressing SQCLC patient samples. 
Strength of activated Akt signals was scored on a scale from zero to 300 in 32 FGFR1-amplified and 45 FGFR1-expressing SQCLC patient samples. Samples with zero $\mathrm{H}$-score showed complete absence of Akt phosphorylation while samples with $300 \mathrm{H}$-score showed the strongest.

Next, I correlated the overall survival of 32 FGFR1-amplified and 45 FGFR1expressing SQCLC patients to their Akt phosphorylation levels. An H-score of $>240$ was used as a cut-off value for strong Akt phosphorylation. Kaplan-Meier survival curves showed an association between increased Akt activation levels and decreased overall survival in both groups. However, the correlation did not reach statistical significance ( $\mathrm{p}$ values $=0.11$ and 0.36 in FGFR1 amplified and expressing samples, respectively) (Figure 31).

A

pAkt in FGFR1-amplified SQCLC

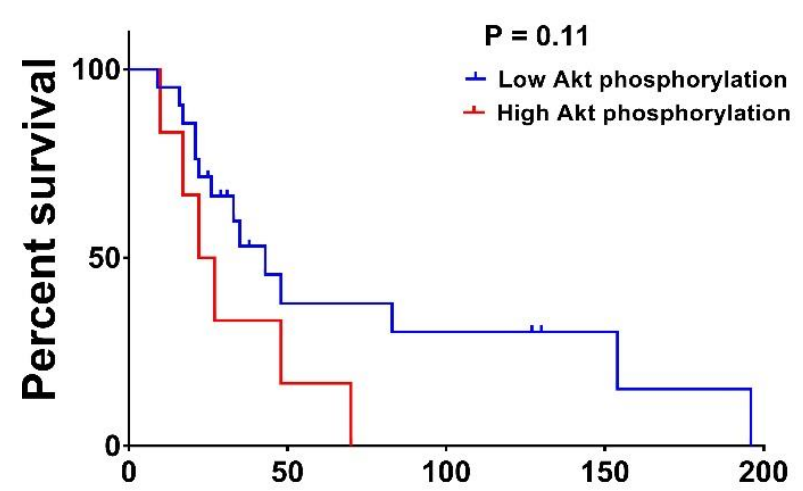

Overall survival time (months)
B

pAkt in FGFR1-expressing SQCLC

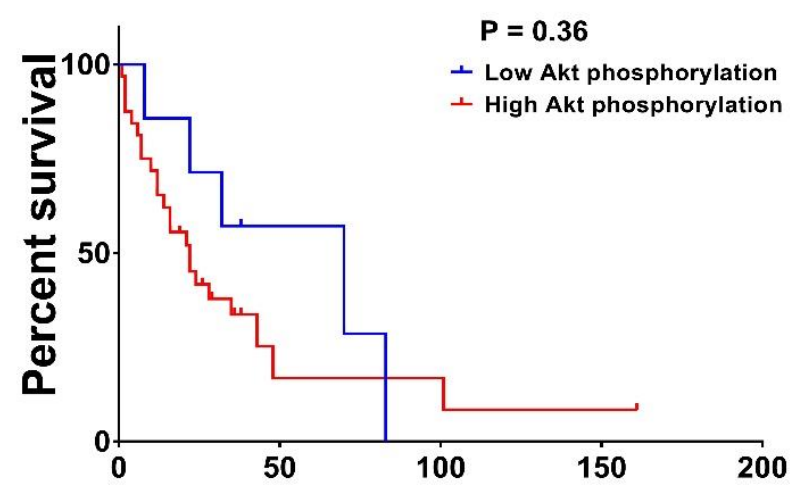

Overall survival time (months)

Figure 31: Survival analysis of FGFR1-amplified and FGFR1-expressing SQCLC patient samples.

Correlation between Akt phosphorylation and overall survival of patients analyzed through KaplanMeier curves.

\subsubsection{Combination of FGFR1 and Akt inhibition}

Next, I tested if Akt activation in $\mathrm{H} 520$ and $\mathrm{H} 1703$ cell lines was connected to their FGFR1 inhibition resistance. With this aim, I inhibited Akt in the seven cell lines in my model. Cell lines were incubated with either the FGFR1 inhibitor AZD4547, the Akt inhibitor AZD5363 or both inhibitors together. The two inhibitors were used in a series of nine increasing concentrations. Inhibitors' concentrations ranged between 0

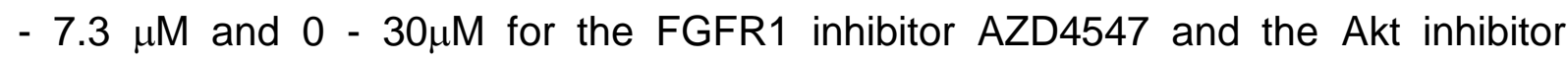
AZD5363, respectively. Cell lines were incubated with the inhibitors for 96 hours and 
analyzed using MTS viability assay. Within the control cell lines HCC15 and H2170, combination inhibition of FGFR1 and Akt showed no distinction in viability compared to Akt inhibition alone. On the other hand, within the two resistant cell lines H520 and $\mathrm{H} 1703$, combination inhibition significantly reduced viability compared to either one of the inhibitors alone. In the three sensitive cell lines combination inhibition reduced viability compared to single inhibitors but to a limited level compared to resistant cell lines. (Figure 32).

\section{Control group}

FGFR1 inhibitor (AZD4547)
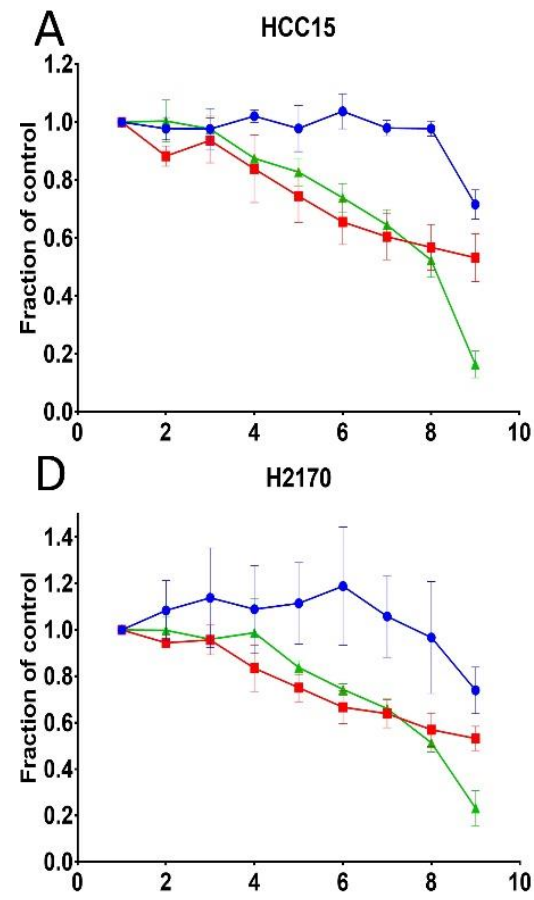

Resistant group

Akt inhibitor (AZD5363)
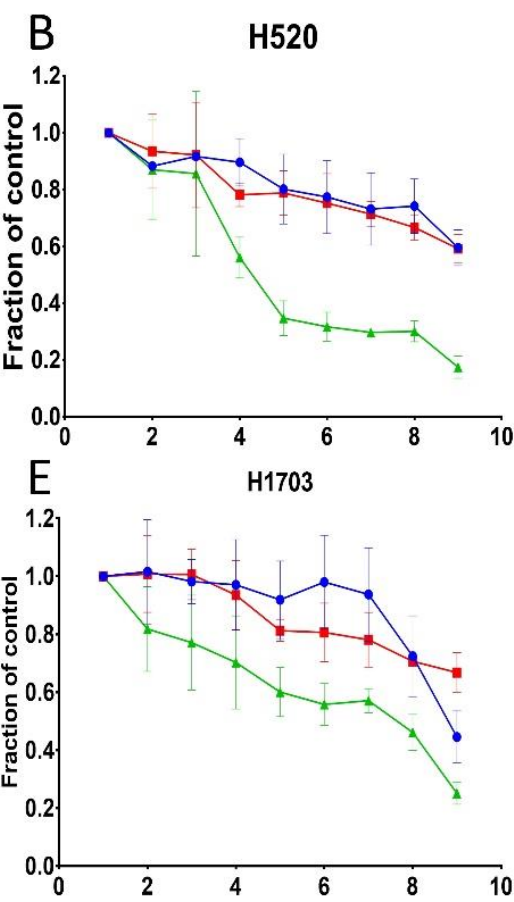

Sensitive group

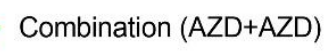

C
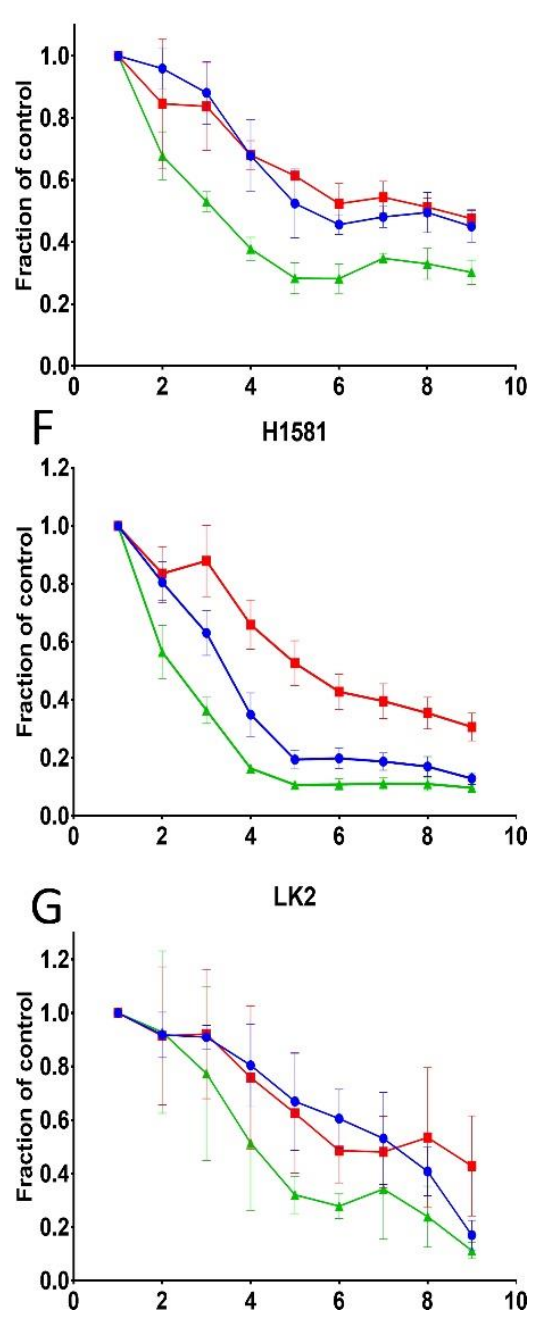

Figure 32: Combination inhibition of FGFR1 and Akt in sensitive and insensitive cell lines FGFR1 inhibition.

Cell lines were treated with nine increasing concentrations of FGFR1 and Akt or both together. The FGFR1 inhibitor AZD4547 was used in concentrations of $0,0.003,0.01,0.03,0.1,0.27,0.8,2.4$ and $7.3 \mu \mathrm{M}$. The Akt inhibitor AZD5363 was used in concentrations of $0,0.5,1,5,10,15,20,20,25$ and 
$30 \mu \mathrm{M}$. Viability was measured using MTS assay after 96 hours of incubation. All experiments were repeated with three biological and three technical replicates.

To investigate the nature of multiple drug interactions, I calculated the combination index value $(\mathrm{Cl})$ according to Chou-Talalay method (CompuSyn software). The method uses the median-effect equation to detect the nature of interactions between two drugs where $\mathrm{Cl}<1, \mathrm{Cl}=1, \mathrm{Cl}>1$ indicate synergistic, additive and antagonistic effects, respectively (Table 4 and Figure 33). Combination indexes showed that combining FGFR1 and Akt inhibition was effective and induced synergism in the two resistant cell lines $\mathrm{H} 1520$ and $\mathrm{H} 1703$.

Table 4: Combination inhibition interactions in $\mathrm{H} 520$ and $\mathrm{H} 1703$ based on Chou-Talalay algorithm.

\begin{tabular}{lccccccc}
\hline $\begin{array}{c}\text { Akt } \\
\text { inhibitor } \\
(\mu \mathrm{M})\end{array}$ & $\begin{array}{c}\text { FGFR1 } \\
\text { inhibitor } \\
(\mu \mathrm{M})\end{array}$ & $\begin{array}{c}\text { Effect in } \\
\mathrm{H} 520\end{array}$ & $\begin{array}{c}\mathrm{Cl} \text { in } \\
\mathrm{H} 520\end{array}$ & $\begin{array}{c}\text { Interaction } \\
\text { in } \mathrm{H} 520\end{array}$ & $\begin{array}{c}\text { Effect in } \\
\mathrm{H} 1703\end{array}$ & $\begin{array}{c}\mathrm{Cl} \text { in } \\
\mathrm{H} 1703\end{array}$ & $\begin{array}{c}\text { Interaction } \\
\text { in } \mathrm{H} 1703\end{array}$ \\
\hline 7.3 & 30.0 & 0.82 & 0.0162 & Synergism & 0.74 & 0.2018 & Synergism \\
2.4 & 25.0 & 0.69 & 0.0536 & Synergism & 0.53 & 0.4471 & Synergism \\
0.8 & 20.0 & 0.7 & 0.0410 & Synergism & 0.42 & 0.5062 & Synergism \\
0.27 & 15.0 & 0.68 & 0.0366 & Synergism & 0.44 & 0.3341 & Synergism \\
0.1 & 10.0 & 0.65 & 0.0317 & Synergism & 0.39 & 0.2595 & Synergism \\
0.03 & 5.0 & 0.43 & 0.0863 & Synergism & 0.29 & 0.2018 & Synergism \\
\hline
\end{tabular}
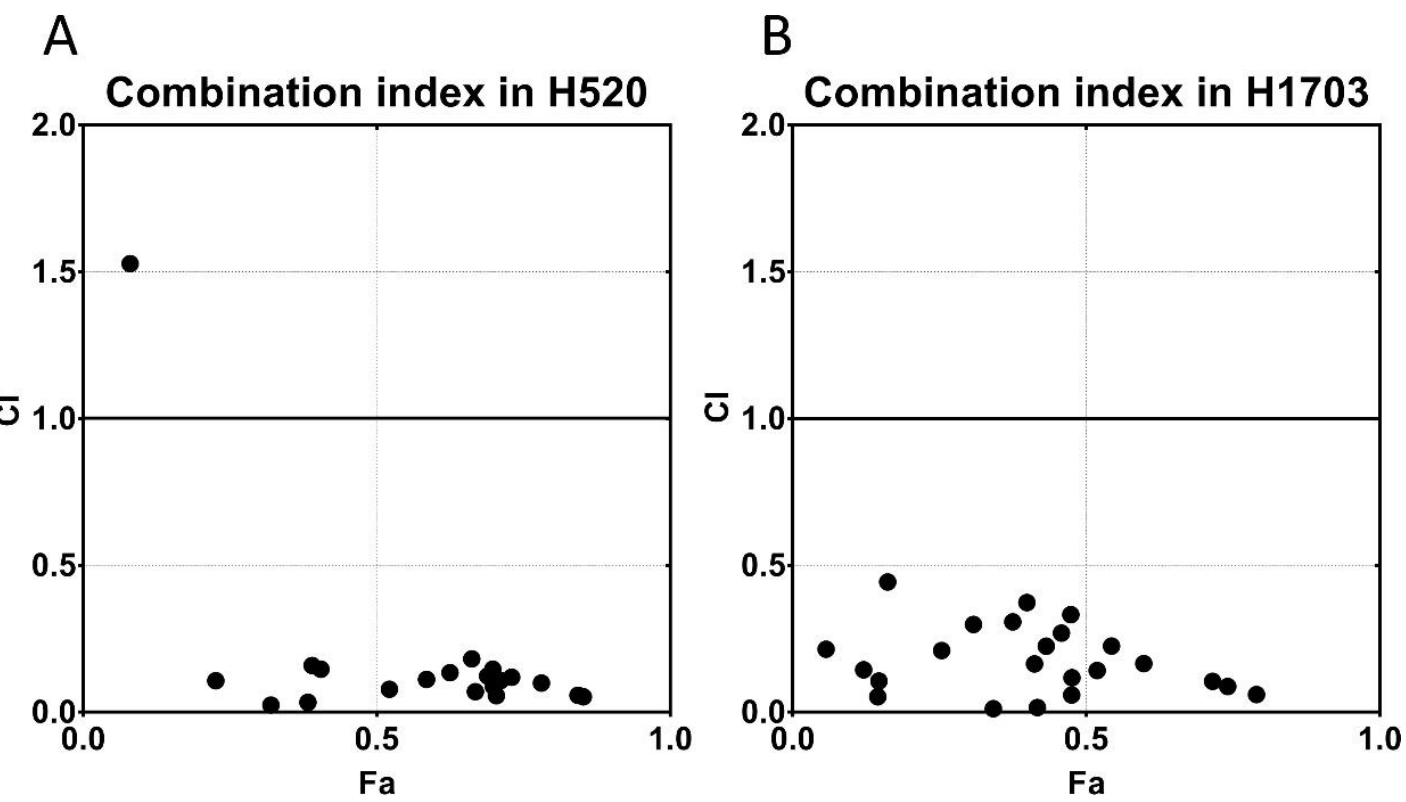
Figure 33: Synergistic effect of FGFR1 and Akt co-inhibition in $\mathrm{H} 520$ and $\mathrm{H} 1703$ cell lines.

$\mathrm{H} 520$ (A) and H1703 (B) resistant cell lines were treated with both the FGFR1 inhibitor AZD4547 and the Akt inhibitor AZD5363 at different concentrations. Fractions of dead cells (Fa) were measured by MTS assay. Combination indexes $(\mathrm{Cl})$ were calculated using CompuSyn software based on ChouTalalay drug interaction algorithm. $\mathrm{Cl}<1$ for synergistic effect, $\mathrm{Cl}=1$ for additive effect and $\mathrm{Cl}>1$ for antagonistic effect.

\subsubsection{Inducing Akt expression and activation in the sensitive cell lines}

Previous results showed that Akt activation might cause resistance to FGFR1 inhibition. I tested this hypothesis through inducing overexpression of activated Akt in the three sensitive cell lines H1581, LK2 and DMS114. Therefore, I transfected the three cell lines with either mammalian expression vector expressing myristoylated Akt1 (pcDNA3-Myr-Akt1) or the same vector without myristoylated Akt1 as a control (pcDNA3). Myristoylation is a lipid modification, which has shown to help membrane localization of Akt and cause its activation (Adam et al., 2007; S. Kim et al., 2001). Successfully transfected cell lines were selected using Geneticin (G418), which was expressed by both vectors. The appropriate concentration of G418 was detected through G418 kill curve for each cell line. Lysates from the three sensitive cell lines were stained for pAkt (Ser473) and analyzed through western blot. Immunostaining confirmed overactivation of Akt in the Myr-Akt1 transfected cell lines compared to either vehicle-vector or control cells (without transfection) (Figure 34). Overactivation of Akt was strong in LK2 and $\mathrm{H} 1581$ cell lines while DMS114 cell line showed minimal overactivation.

\section{LK2 \\ H1581 \\ DMS114}

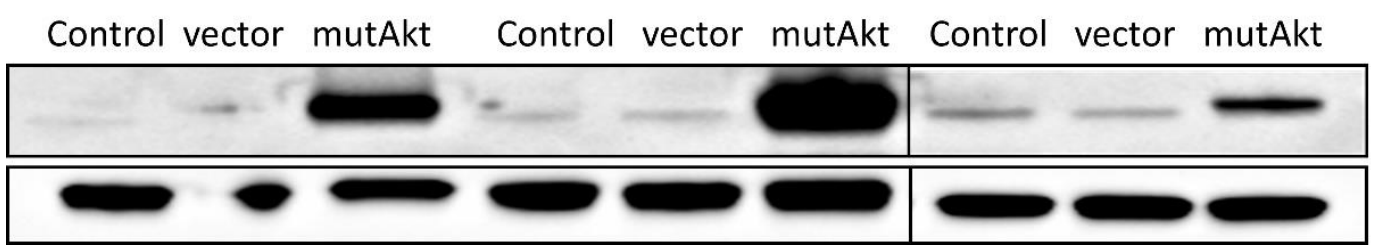

pAkt Ser347

PARK7

Figure 34: Immunoblotting of phosphorylated Akt in control and constitutively active-Akt cell lines.

Activated Akt levels were measured using western blot in control cells, empty vector-transfected cell lines and Myr-Akt1 expressing vector-transfected cell lines. 
The effect of Akt overactivation in the three sensitive cell lines was evaluated through MTS viability assay. In the cell line $\mathrm{H} 1581$, induction of pAkt significantly reduced its sensitivity to the FGFR1 inhibitor AZD4547 compared to cells transfected with vehicle vector. In LK2 cell line, induction of pAkt also reduced the sensitivity to FGFR1 inhibition albeit to a lesser extent. In DMS114 cell line, there was no change in sensitivity between pAkt-induced cells and control cells (Figure 35).

A

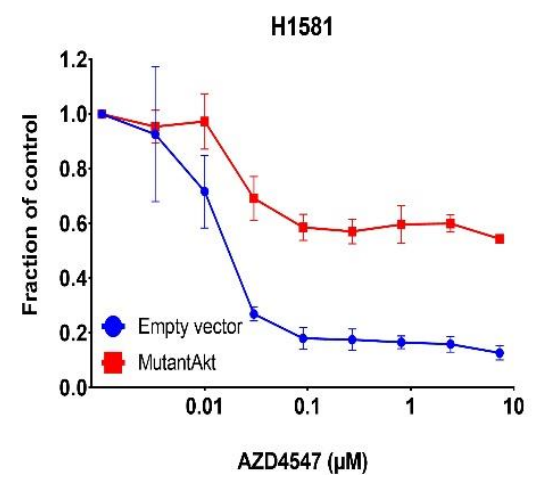

B

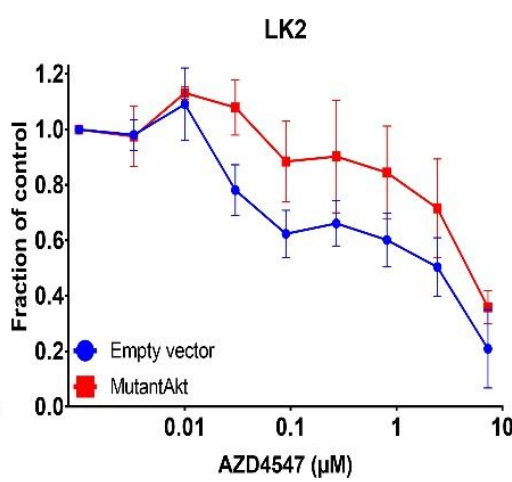

C

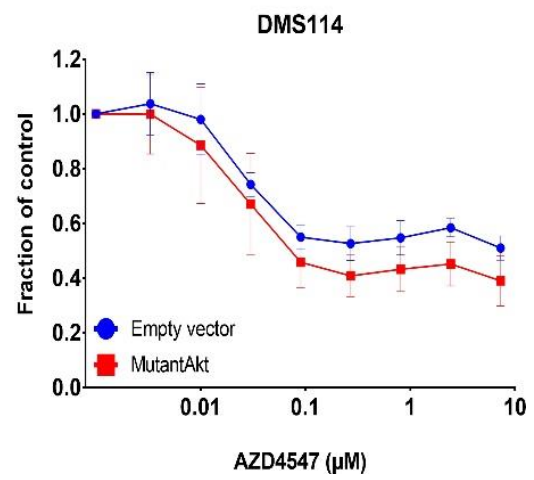

Figure 35. Sensitivity to FGFR1 inhibition in mutationally activated Akt cell lines.

Control cells (transfected with an empty vector) and pAkt overexpressing cells (transfected with pAktexpressing vector) were treated with the FGFR1 inhibitor AZD4547 for 96 hours. The FGFR1 inhibitor AZD4547 was used in concentrations of $0,0.003,0.01,0.03,0.1,0.27,0.8,2.4$ and $7.3 \mu \mathrm{M}$ Viability of the cells were measured using MTS viability assay. Experiments were conducted in triplicates.

Combination inhibition of FGFR1 and Akt showed successful rescue of sensitivity to FGFR1 inhibition in the cell lines H1581 and LK2. No change in sensitivity to FGFR1 inhibition was detected between Myr-Akt1 and empty vector transfected cells in DMS114 cell line (Figures 36). Combination indexes, calculated through ChouTalalay method, showed synergistic effect between the two inhibitors, which elevated in Myr-Akt1 expressing cell lines, i.e. decrease in $\mathrm{Cl}$ values (Figures 37 A-F). 
FGFR1 inhibitor (AZD4547)

A
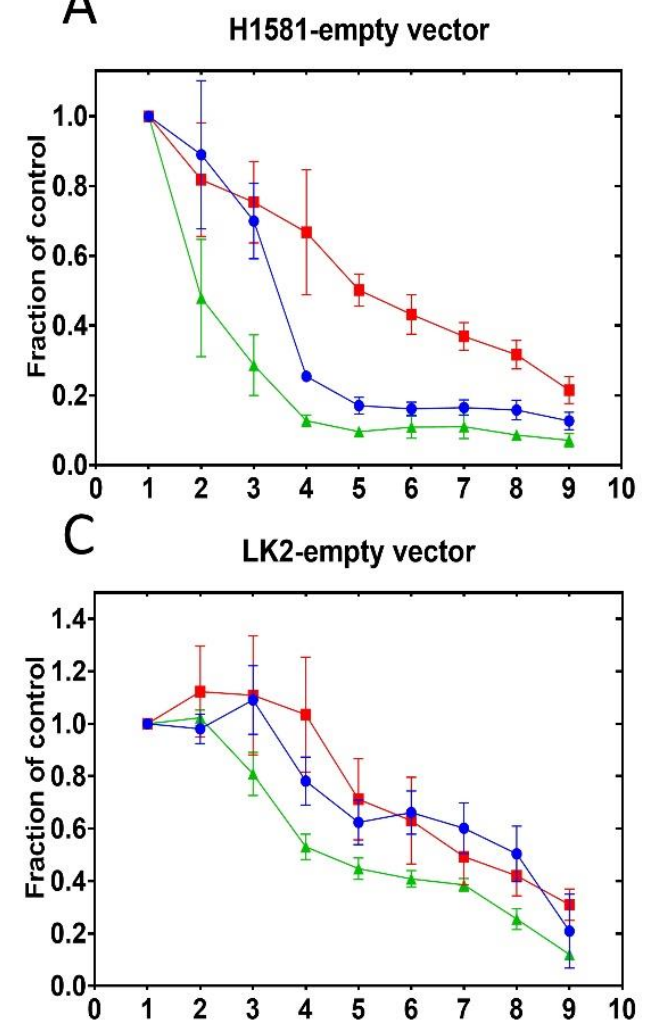

$\mathrm{E}$

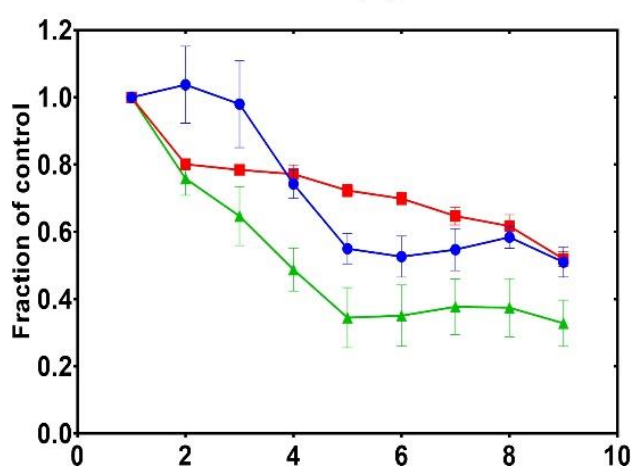

Akt inhibitor (AZD5363)

B

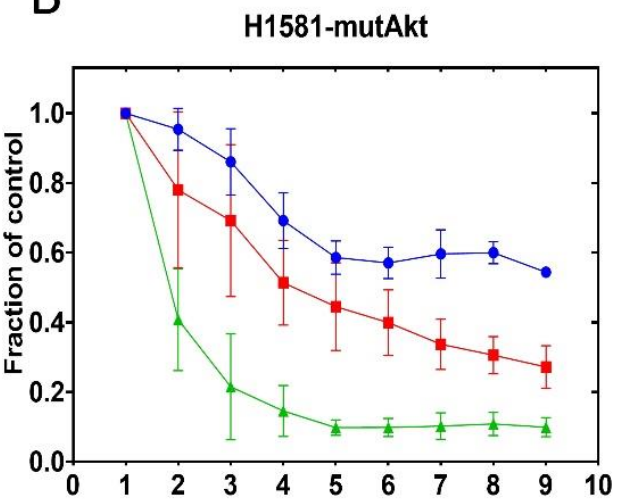

D LK2-mutAkt

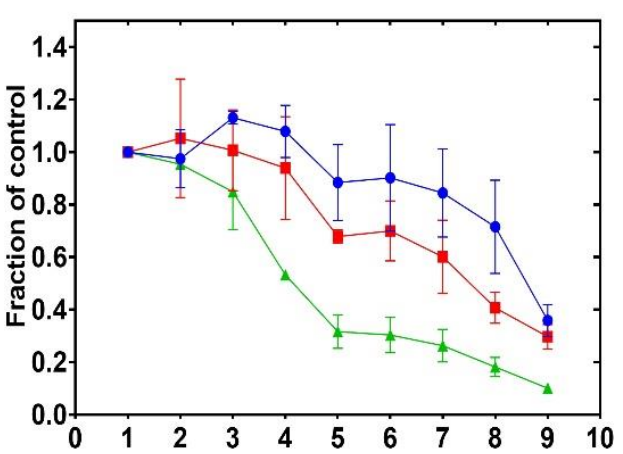

F DMS114-mutAkt

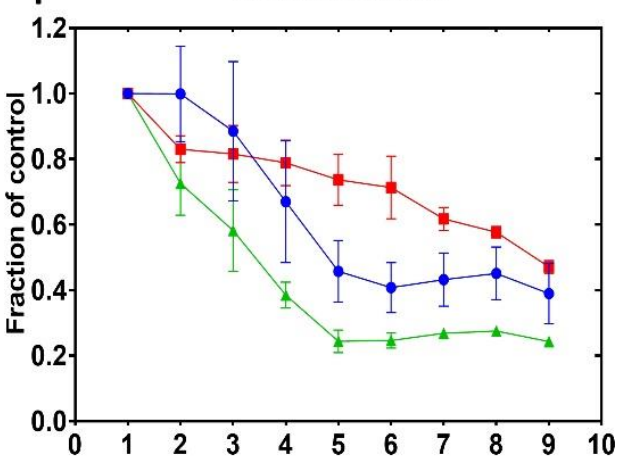

Figure 36: Combination inhibition of FGFR1 and Akt in control and MyrAkt1 expressing cell lines.

Cell lines were treated with nine increasing concentrations of FGFR1 and Akt or both together. The FGFR1 inhibitor AZD4547 was used in concentrations of 0, 0.003, 0.01, 0.03, 0.1, 0.27, 0.8, 2.4 and $7.3 \mu \mathrm{M}$. The Akt inhibitor AZD5363 was used in concentrations of $0,0.5,1,5,10,15,20,25$ and 30 $\mu \mathrm{M}$. MTS viability assay was measured after 96 hours incubation. Experiments were conducted in triplicates. 


\section{Combination index}
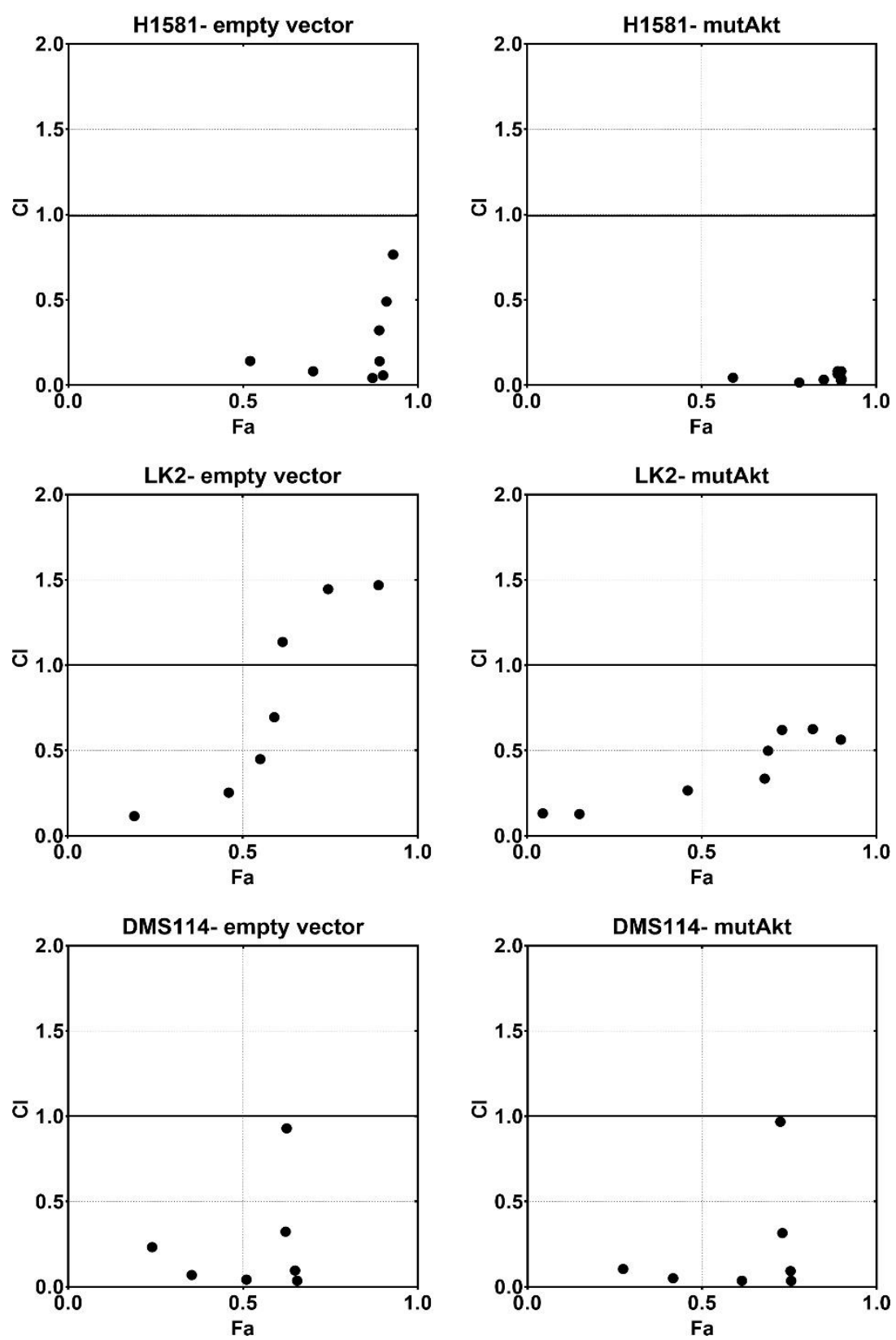

Figure 37: Combination index values in control and mutationally activated Akt cell lines.

H1581, LK2 and DMS114 cell lines, which express mutant Myr-Akt1, were treated with both the FGFR1 inhibitor AZD4547 and the Akt inhibitor AZD5363 at different concentrations. Fractions of dead cells $(\mathrm{Fa})$ were measured by MTS assay. Combination indexes $(\mathrm{Cl})$ were calculated using CompuSyn software based on Chou-Talalay drug interaction algorithm. $\mathrm{Cl}<1$ for synergistic effect, $\mathrm{Cl}=1$ for additive effect and $\mathrm{Cl}>1$ for antagonistic effect. 


\subsubsection{Inducing resistance to FGFR1 inhibition in sensitive cell lines}

Exposure to molecular targeted inhibitors can induce resistance to these inhibitors in cancer patients. In order to study mechanisms of acquired resistance, I induced FGFR1 inhibition resistance in the three sensitive cell lines H1581, LK2 and DMS114. Resistance induction was possible through incubating the three cell lines with high concentration of the FGFR1 inhibitor AZD4547 (5 $\mu \mathrm{M})$ over a long period. During resistance induction, cells were treated with several cycles of either fresh medium only or medium with inhibitor for three days. The level of sensitivity of the cells was assayed via MTS viability assay. High level of resistance was achieved after a period of four months (Figure 38).
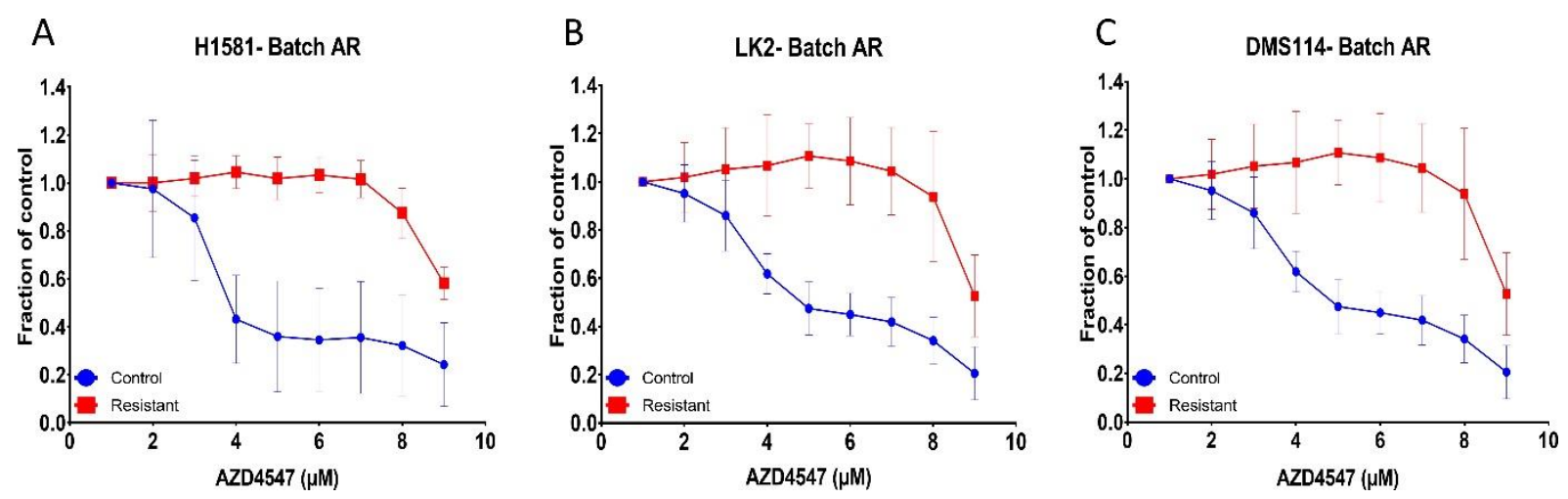

Figure 38: Acquired resistance to FGFR1 inhibition in the three sensitive cell lines.

MTS viability assay compared sensitivity of parental cell lines and their resistant counterparts to the FGFR1 inhibitor AZD4547. Cells were incubated with the FGFR1 inhibitor for 96 hours. Experiments were conducted in triplicates.

Altered signaling in the resistant cell lines was tested through western blot and compared to signaling in control cell lines. Repeated detection of signaling in the resistant cell lines showed a switch between activated and deactivated pathways within the same resistant cell line. Western blot was repeated at least three times for each cell line over a period of one month and the unstable behavior of cell signaling was detected repeatedly. Activated Akt was one of the effectors, which were elevated at certain time point and reduced afterwards in the same resistant cell line compared to control cells. One explanation of the unstable signaling is the multiclonal behavior of the resistant cell lines where each clone developed its own resistant mechanism (Figure 39). 


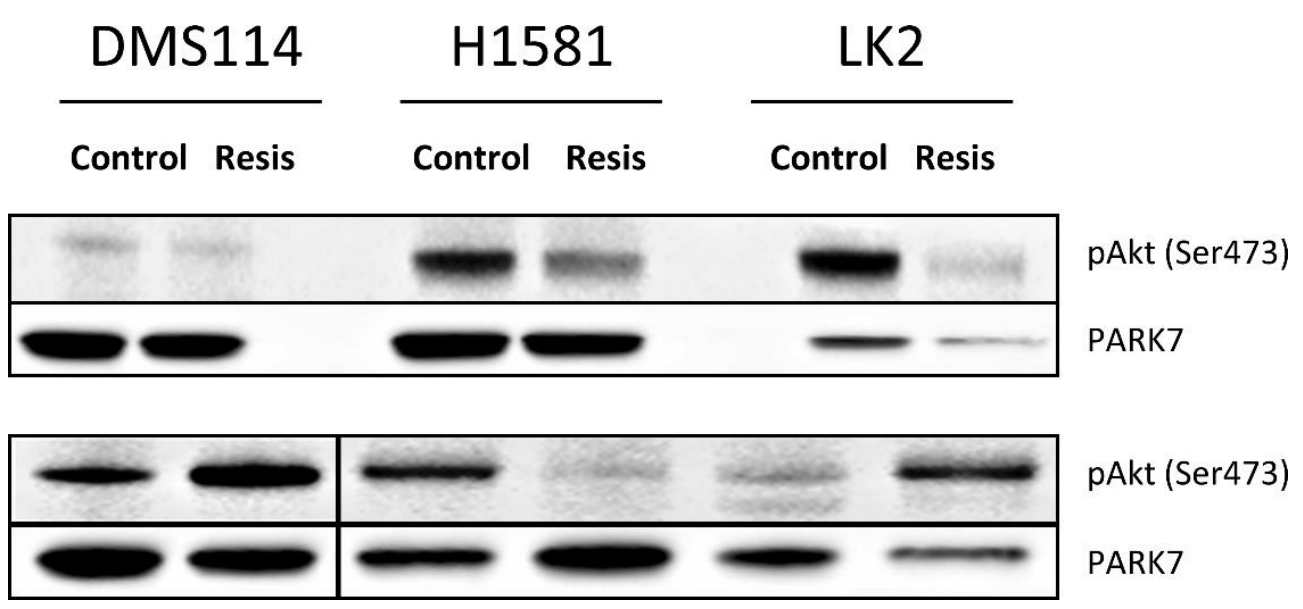

Figure 39: Western blot analysis of Akt phosphorylation levels in acquired resistant cell lines. Levels of pAkt were measured in the three parental cell lines DMS114, H1581 and LK2 and their acquired resistant counterparts in two different biological replicates. Replicates were measured two weeks apart. PARK7 was used as a loading control.

\subsubsection{Inducing resistance to FGFR1 inhibition in single clones of sensitive cell lines}

To test the hypothesis of multiclonal resistance mechanisms and to study mechanisms of resistance to FGFR1 inhibition in lung cancer, I sorted the sensitive cell lines into single clones. The three sensitive parental cell lines H1581, LK2 and DMS114 were sorted into single cells using FACS sorter on 96-well plates. Single cells were incubated with normal growth medium until the cells were stabilized and slightly expanded. Afterwards, Clones were incubated with several cycles of either fresh medium or medium with $5 \mu \mathrm{M}$ of the FGFR1 inhibitor AZD4547 to induce the resistance.

Cycles of FGFR1 inhibition lasted for five days while cycles of recovery with fresh medium lasted for 20 days. Unlike batch cell lines, single clones were very sensitive to any medium change as well as FGFR1 inhibition. Sensitivity of the single clones had elongated the duration of each recovery cycle from 3 days in batch cell lines to 20 days in single clones. The process of inducing resistance in single clones lasted for ten months. Resistance to the FGFR1 inhibitor AZD4547 was detected through MTS viability assay compared to parental cell lines.

In $\mathrm{H} 1581$ cell line, I sorted 480 single cells from eight 96-well plates, in which 150 clones (31.2\%) survived the sorting. Out of the 150 single clones of H1581 cell line, 
14 (9.3) clones developed resistance to FGFR1 inhibition. In DMS114 cell line, 420 single cells were sorted on seven 96-well plates, in which 178 clones (42.3\%) survived. Seventeen single clones (9.6\% of total single clones) developed resistance to the FGFR1 inhibitor AZD4547. In LK2 cell line, 540 cells were sorted on nine 96well plates, in which $74(13.7 \%)$ cells survived. Percentage of developing resistance to FGFR1 inhibition was $22.9 \%$ (17 out of 74 single clones) in LK2 cell line (Table 5).

Next, I investigated the altered signaling pathways in the resistant clones compared to their sensitive parental cell lines through western blot. In this regard, I checked and compared expression levels of FGFR1, phosphorylated Akt, basal Akt, phosphorylated ERK and basal ERK proteins. PARK7 was used as a loading control.

Western blot showed overexpression of FGFR1 protein expression in 5 out of 14 $(35.7 \%), 9$ out of $17(52.9 \%)$ and 6 out of $17(35.3 \%)$ resistant single clones of H1581, DMS114 and LK2 cell lines, respectively. Overactivation of Akt was detected in 3 out of $14(21.4 \%), 5$ out of $17(29.4 \%)$ and 1 out of $17(5.9 \%)$ resistant single clones of H1581, DMS114 and LK2 cell lines, respectively. Expression of phosphorylated ERK was elevated in 2 out of 14 (14.3\%), 1 out of 17 (5.9\%) and 4 out of $17(25.5 \%)$ resistant single clones of H1581, DMS114 and LK2 cell lines, respectively (Table 5 and Figure 40).

Table 5: Statistics of inducing resistance to the FGFR1 inhibitor AZD4547.

\begin{tabular}{lccc}
\hline Cell line & H1581 & DMS114 & LK2 \\
\hline Plates sorted & 8 & 7 & 9 \\
Cells sorted & 480 & 420 & 540 \\
Clones survived & $150(31.2 \%)$ & $178(42.3 \%)$ & $74(13.7 \%)$ \\
Clones resistant & $14(9.3 \%)$ & $17(9.6 \%)$ & $17(22.9 \%)$ \\
FGFR1 overexpression & $5(35.7 \%)$ & $9(52.9 \%)$ & $6(35.3 \%)$ \\
pAkt activation & $3(21.4 \%)$ & $5(29.4 \%)$ & $1(5.9 \%)$ \\
pERK activation & $2(14.3 \%)$ & $1(5.9 \%)$ & $4(25.5 \%)$ \\
\hline
\end{tabular}

AZD4547: FGFR1 inhibitor 
A H1581 Resistant Clones

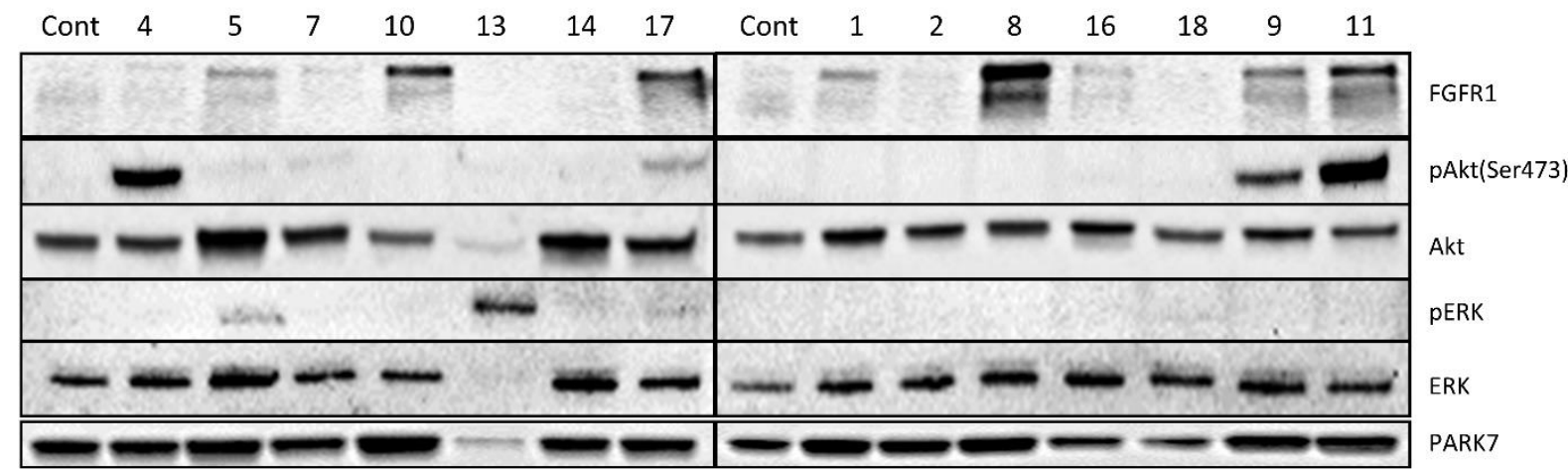

B

\section{LK2 Resistant Clones}

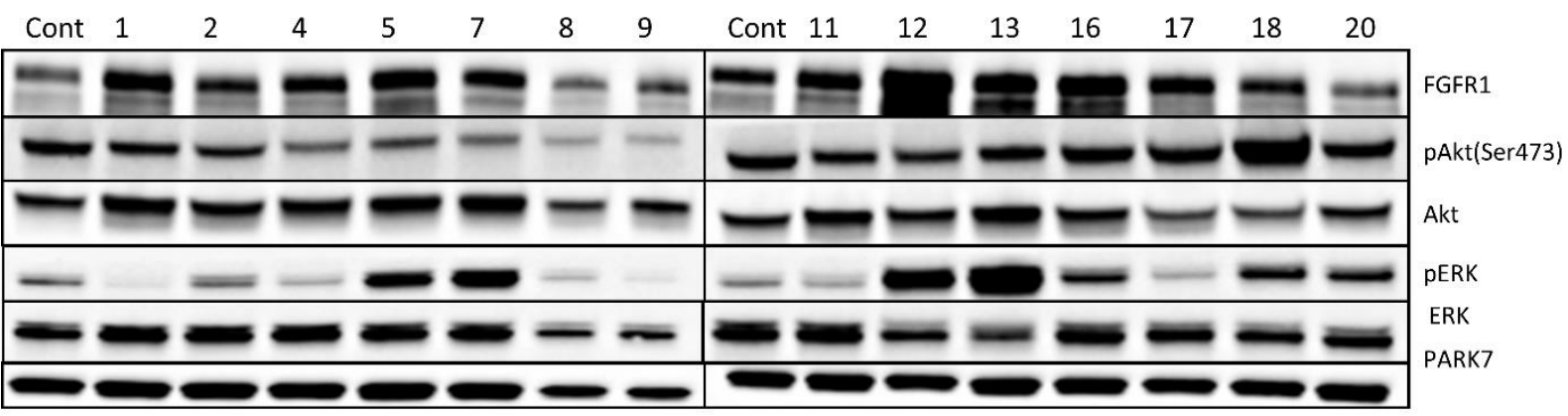

C

DMS114 Resistant Clones

\begin{tabular}{|c|c|c|c|c|c|c|c|c|c|c|c|c|c|c|}
\hline Cont & 1 & 2 & 4 & 5 & 6 & 8 & 9 & 10 & 12 & 13 & 14 & 15 & 17 & 19 \\
\hline & $\sin 8$ & 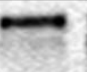 & 5 & 5 & 5 & End & 20 & tiones & -3 & $\longrightarrow$ & $\rightarrow$ & 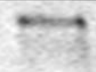 & $\rightarrow$ & $\overrightarrow{x+4}$ \\
\hline kapos & noser & & Wiow & & Whow & بnen & (1) & & & nees & 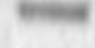 & 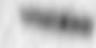 & & \\
\hline$=$ & $=$ & 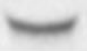 & $=$ & - & $=$ & 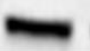 & 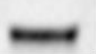 & & & & & & 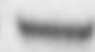 & 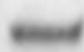 \\
\hline & S & tent & buse? & $=$ & 60 & |lase & Sack & & 4064 ? & & 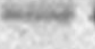 & 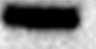 & 6040 & \\
\hline 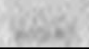 & nave & 26.4 & 68 & & $\sqrt{4}+4$ & 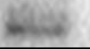 & 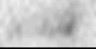 & -4 & 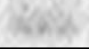 & sinet & suck & 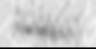 & & \\
\hline
\end{tabular}

Figure 40: Western blot analysis showing altered signaling in FGFR1 inhibition resistant clones compared to parental controls.

Levels of FGFR1, pAkt, Akt, pERK and ERK were tested in single cell resistant clones of H1581 (A), LK2 (B) and DMS114 (C) cell lines and compared to their parental controls (Cont.). PARK7 was used as a loading control.

\subsubsection{Combination inhibition of FGFR1 and Akt in resistant single clones}

In order to investigate mechanisms of resistance to FGFR1 inhibition in depth, I focused my efforts on studying overactivated Akt as a mechanism of resistance. With this aim, I selected the three resistant clones numbered as 4, 9 and 11 of the $\mathrm{H} 1581$ cell line for further studies. The three resistant clones H1581-4R, H1581-9R and 
H1581-11R showed elevation of pAkt as one mechanism of resistance to the FGFR1 inhibitor AZD4547.

In order to test the dependency of the resistant clones H1581-4R, H1581-9R and $\mathrm{H} 1581-11 \mathrm{R}$ on Akt signaling, I inhibited Akt alongside with FGFR1 in these clones. Firstly, I incubated the resistant clone $\mathrm{H} 1581-11 \mathrm{R}$ with nine increasing concentrations of either FGFR1 inhibitor AZD4547 alone, Akt inhibitor AZD5363 alone or both inhibitors together for 96 hours. The nine different concentrations allowed me to find the effective concentration of the two inhibitors as well as measuring the combination index among different combinations. Comparison between viability of H1581-11R and parental cell line confirmed the resistance to FGFR1 inhibition, while response to Akt inhibition was not altered. Combination inhibition in H1581-11R and parental H1581 cell line showed significant reduction in viability in the resistant clone compared to parental cells. Chou-Talalay equations showed synergistic effect of the combination inhibition in five different combination concentrations (Figures 41 and 42).
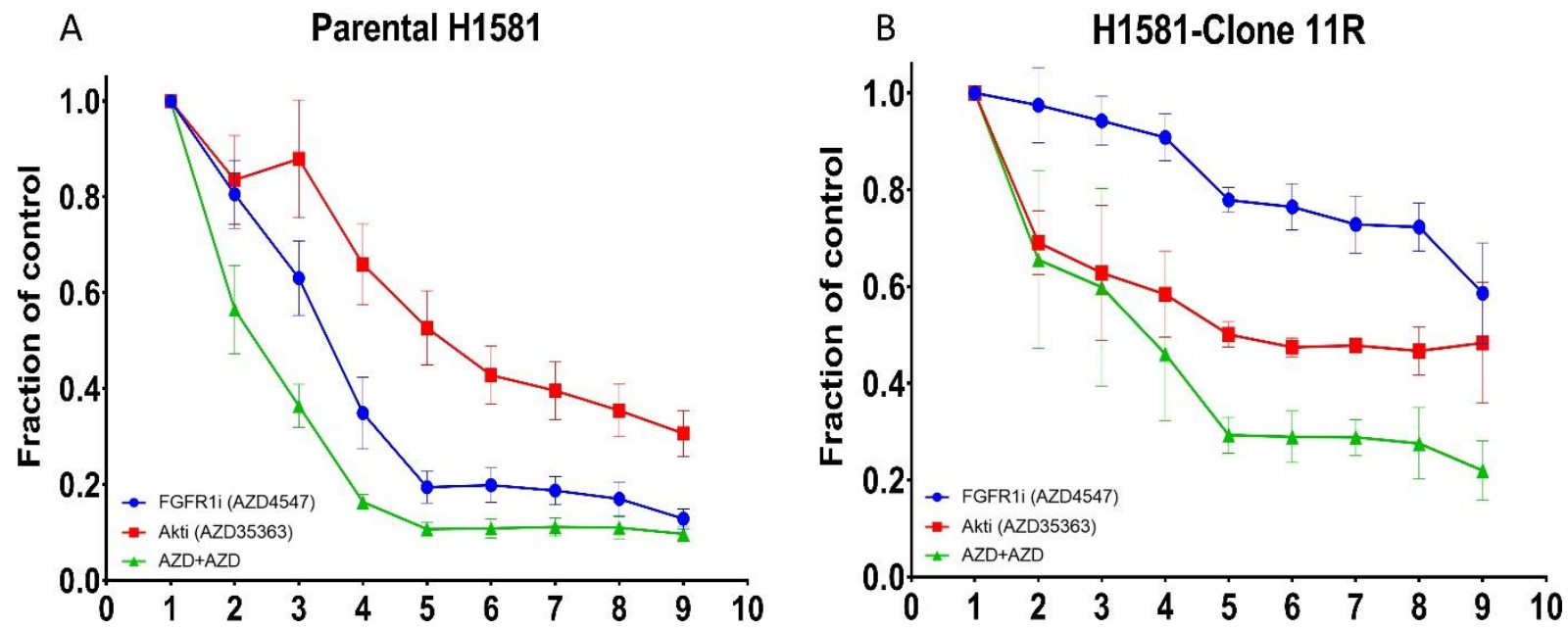

Figure 41: Synergistic effect of FGFR1 and Akt co-inhibition in the FGFR1 inhibition resistant clone H1581-11R.

Resistant clone number 11 of $\mathrm{H} 1581$ cell line was incubated with either the FGFR1 inhibitor AZD4547 alone, the Akt inhibitor AZD5363 alone or both inhibitors together. Inhibitors were used in nine increasing concentrations. The FGFR1 inhibitor AZD4547 was used in concentrations of 0, 0.003, $0.01,0.03,0.1,0.27,0.8,2.4$ and $7.3 \mu \mathrm{M}$. The Akt inhibitor AZD5363 was used in concentrations of 0 , $0.5,1,5,10,15,20,20,25$ and $30 \mu \mathrm{M}$. MTS viability assay was measured after 96 hours incubation. Experiments were conducted in triplicates. 


\begin{tabular}{lllcl}
\hline Akti & FGFR1i & Effect & \multicolumn{1}{c}{ Cl } & Interaction \\
\hline 30.0 & 7.3 & 0.78 & 0.03608 & Synergism \\
25.0 & 2.4 & 0.72 & 0.04801 & Synergism \\
20.0 & 0.8 & 0.71 & 0.03499 & Synergism \\
15.0 & 0.27 & 0.71 & 0.02307 & Synergism \\
10.0 & 0.1 & 0.7 & 0.01789 & Synergism \\
5.0 & 0.03 & 0.54 & 0.16460 & Synergism \\
\hline
\end{tabular}

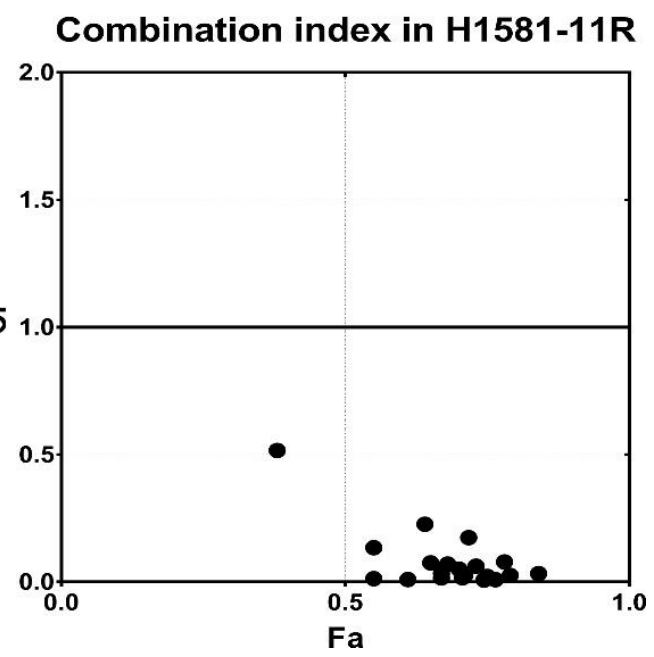

Figure 42: Combination index values indicated type of interaction between the FGFR1 inhibitor AZD4547 and the Akt inhibitor AZD5363 in H1581-11R resistant clone.

The effect of combination inhibition was evaluated through MTS viability assay after 96 hours incubation. H1581-11R resistant clone was treated with both the FGFR1 inhibitor AZD4547 and the Akt inhibitor AZD5363 at different concentrations (A). Fractions of dead cells ( $\mathrm{Fa}$ ) were measured by MTS assay (B). Combination indexes $(\mathrm{Cl})$ were calculated using CompuSyn software based on ChouTalalay drug interaction algorithm. $\mathrm{Cl}<1$ for synergistic effect, $\mathrm{Cl}=1$ for additive effect and $\mathrm{Cl}>1$ for antagonistic effect.

In order to test the effect of combination inhibition of FGFR1 and Akt in the two resistant clones H1581-4R and H1581-9R, I incubated these clones with either inhibitors or combination of them for 96 hours. The FGFR1 inhibitor AZD4547 was used at concentration of $7.3 \mu \mathrm{M}$ while the Akt inhibitor AZD5363 was used at concentration of $30 \mu \mathrm{M}$. MTS viability assay alongside with viable cell count using Muse cell analyzer showed resistance of the three clones to the FGFR1 inhibitor (AZD4547) compared to parental H1581 cell line (Figure 43). Combination inhibition showed synergistic effect of combining the FGFR1 inhibitor AZD4547 and the Akt inhibitor AZD5363 in the three single clones H1581-4R, H1581-9R and H1581-11R (Figure 44). 

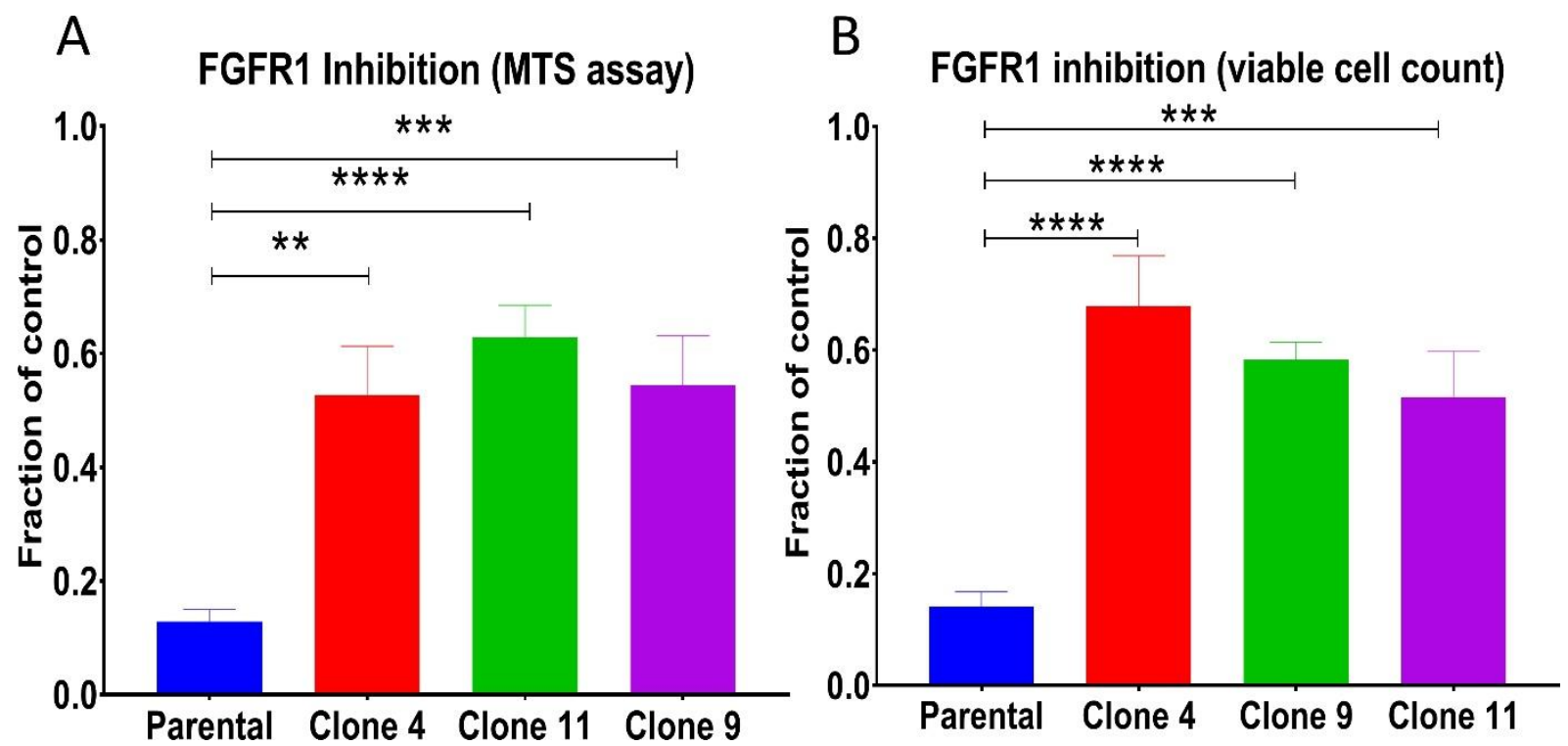

Figure 43: Effect of FGFR1 inhibition in parental and resistant clones of $\mathrm{H} 1581$ cell line.

MTS viability assay compared viability of parental $\mathrm{H} 1581$ cell line and the resistant single clones upon treatment with the FGFFR1 inhibitor AZD4547. The assay was measured after 96 hours incubation. The AZD4547 inhibitor was used at concentration of $7.3 \mu \mathrm{M}$. Experiments were conducted in triplicates. $P$ values were denoted as $n s=p>0.05,{ }^{*}=p \leq 0.05,{ }^{* *}=p \leq 0.01,{ }^{* * *}=p \leq 0.001$ and ${ }^{* * * *}=p$ $\leq 0.0001$.

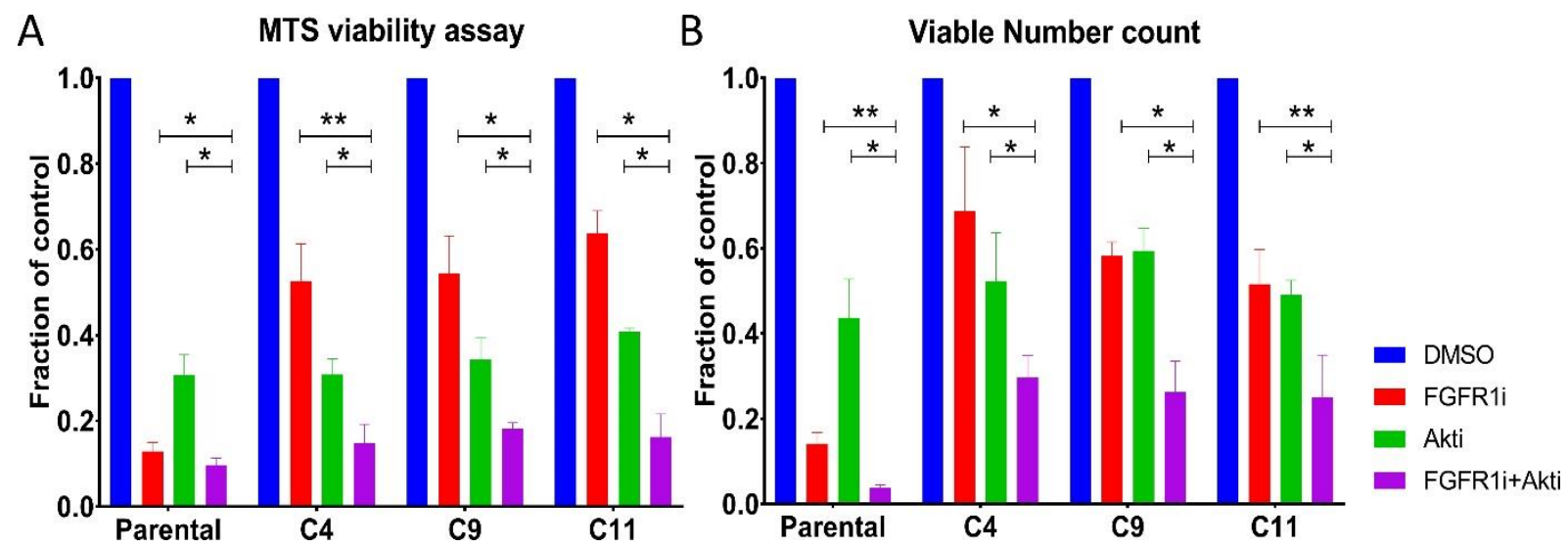

Figure 44: Synergistic effect of FGFR1 TKI combined with Akt inhibitor in resistant clones.

The parental H1581 cell line and the three resistant clones H1581-4R, H1581-9R and H1581-11R were treated with either the FGFR1 inhibitor AZD4547 alone, the Akt inhibitor AZD5363 alone or both inhibitors together. The AZD4547 inhibitor was used at concentration of $7.3 \mu \mathrm{M}$. The AZD5363 inhibitor was used at concentration of $30 \mu \mathrm{M}$. MTS assay was measured after 96 hours incubation. Experiments were conducted in triplicates. $P$ values were denoted as $n s=p>0.05,{ }^{*}=p \leq 0.05,{ }^{* *}=p$ $\leq 0.01,{ }^{* * *}=p \leq 0.001$ and ${ }^{* * *}=p \leq 0.0001$.

In order to understand mechanism of resistance in the single clones more, I blotted proteins out of the three clones alongside with the parental $\mathrm{H} 1581$ control after 
incubation with either DMSO as a control or $0.5 \mu \mathrm{M}$ AZD4547 for 3 hours on membranes and stained them against the main effectors in FGFR1 signaling (Figure 45). Western blotting showed strong activation of Akt in the three resistant clones. The downstream Akt proteins, PRAS40 and FOXO1/3, were strongly activated in the three clones as well. PTEN expression was down regulated in clones $\mathrm{C} 9$ and $\mathrm{C} 11$. Decline of ERK and MEK activation was noticed in at least two of the resistant clones (C9 and C11).

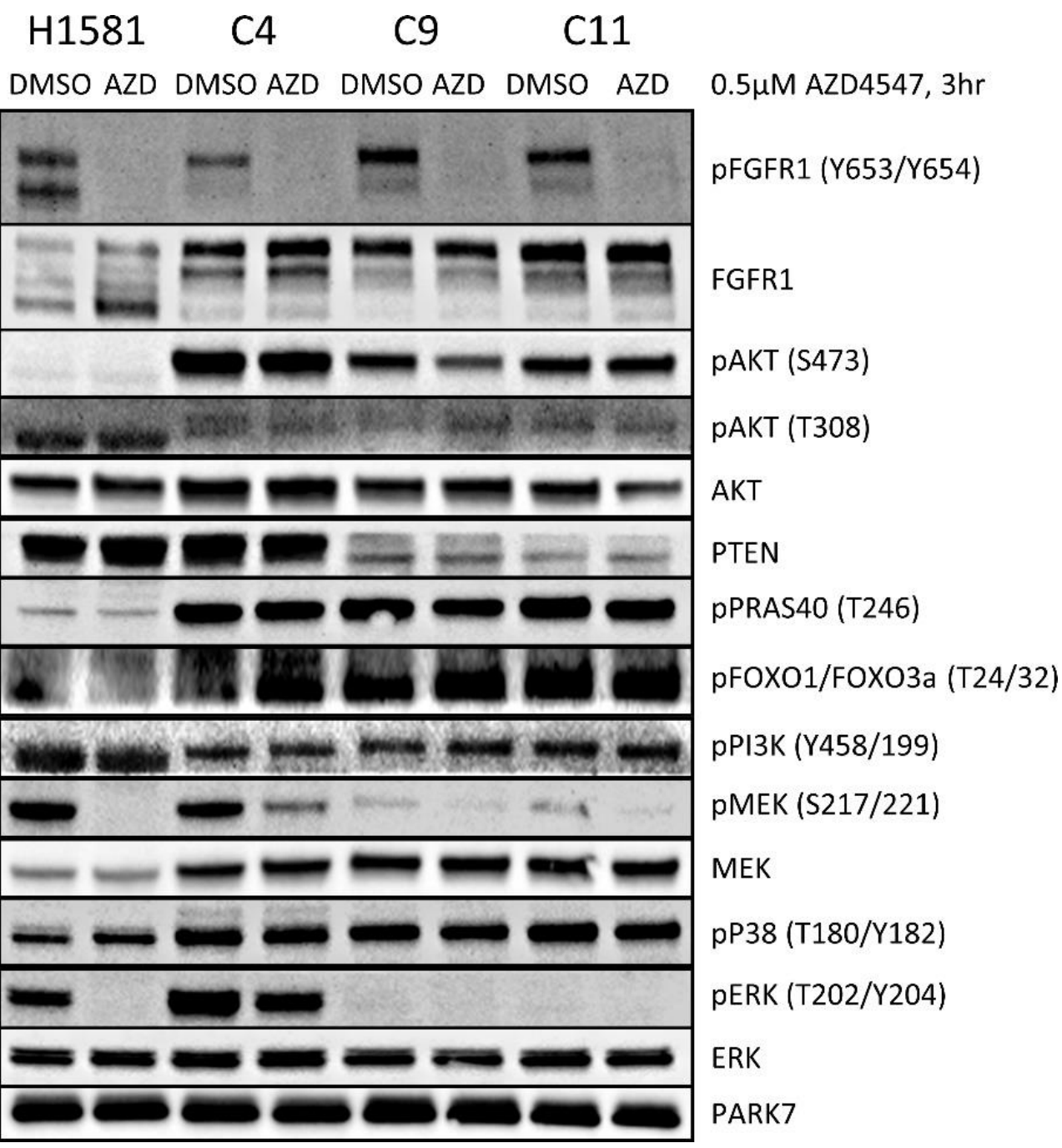

Figure 45: Activated signaling pathway in induced resistance single clones of $\mathrm{H} 1581$ cell line Western blot showed regulation of FGFR1 signaling pathway in the parental $\mathrm{H} 1581$ cell line compared to the three induced resistance single clones C4, 9 and 11. Cells were incubated with either DMSO or $0.5 \mu \mathrm{M}$ AZD4547 for 3 hours and then lysed.

RNA sequencing using CTL directed panel showed increase in FGFR1 RNA expression and decrease in GNAS RNA expression as the differences between the 
three resistant clones and the parental control cell line (Figure 46). EGFR, ERBB2, Akt1, BRAF, MET, KRAS and PIK3CA RNA expression were not altered between the resistant clones and the control.

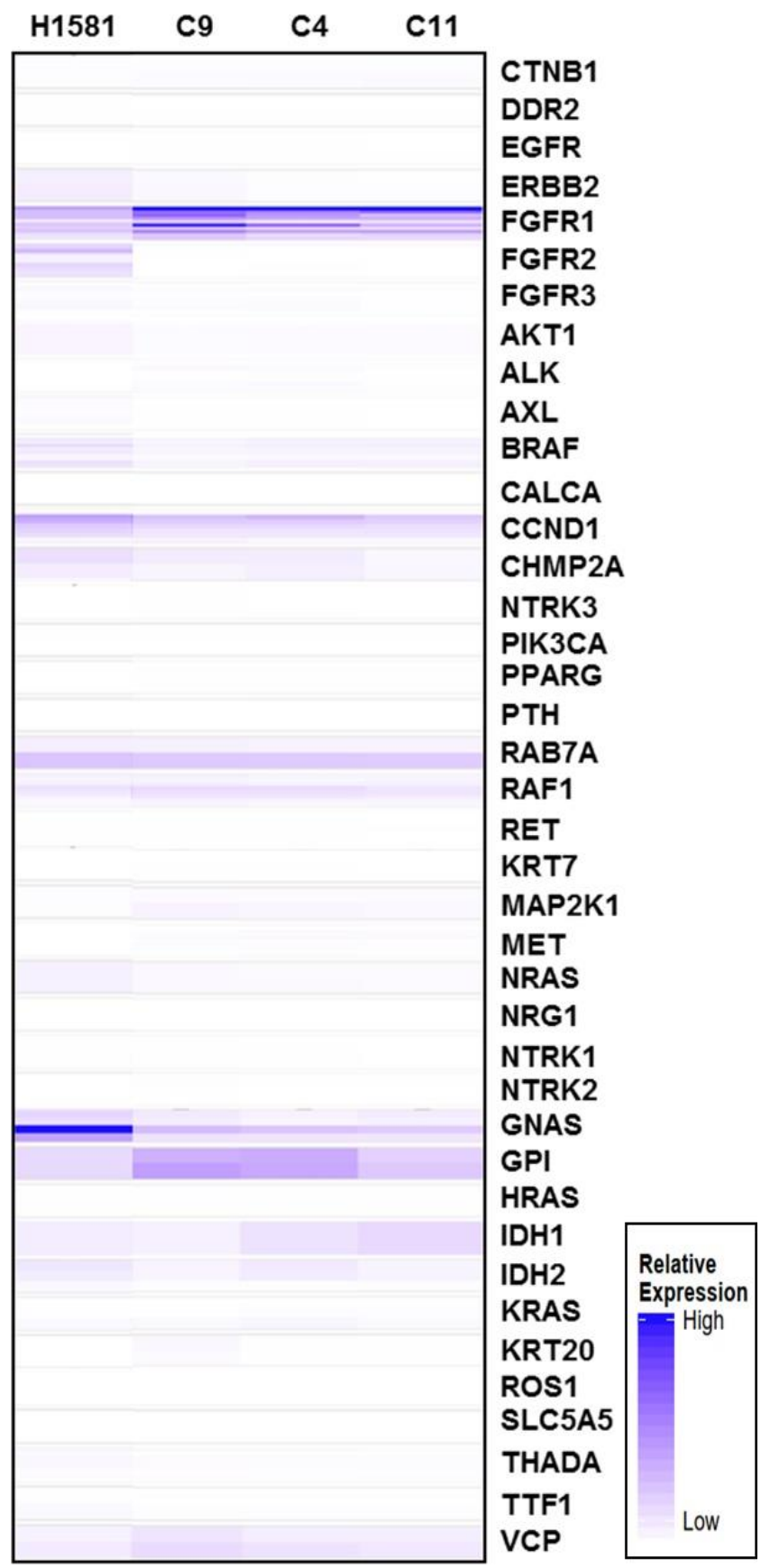

Figure 46: RNA sequencing showed protein expression levels among resistant clones.

Archer thyroid and lung cancer directed panel was used to compare protein expression levels among resistant single clones and parental control of $\mathrm{H} 1581$ cell line. 


\subsection{Phosphoproteomic analysis of FGFR1 inhibition resistance in lung cancer cell lines}

\subsubsection{Sample preparation for phosphoproteomic analysis}

In order to investigate the mechanism of resistance to FGFR1 inhibition in depth, with special focus on the role of Akt in FGFR1 resistance, I conducted a phosphoproteomic analysis using mass spectrometry. The experiment was designed to investigate mechanisms of resistance to FGFR1 inhibition in three different resistant groups and one sensitive control group. The first resistant group included the intrinsic resistant cell line H520. The second resistant group included the H1581 cell line with mutationally activated Akt (H1581-mutAkt). The last resistant group included the two induced resistance single clones with elevated Akt activation $\mathrm{H} 1581$ 9R and H1581-11R. The sensitive control group included the parental H1581 cell line. To expand my understanding of the resistance mechanisms, I compared all cell lines under two different conditions. The first condition was treatment with $0.5 \mu \mathrm{M}$ of the FGFR1 inhibitor AZD4547 and the second condition was treatment with the same amount of DMSO.

To ensure accurate measurement and comparison of proteins from different sources on mass spectrometry, samples had to be mixed in the same ratios and measured together. In order to mix samples together, I labelled the samples with SILAC metabolic labelling. AZD4547-treated cells were labelled with heavy SILAC amino acids (Lysine +8 and Arginine +10 ), while DMSO-treated cells were labelled with medium SILAC amino acids (Lysine +4 and Arginine +6 ).

To compare cell lines from different groups, I spiked a standard control in each of the samples. The standard control consisted of equal amounts of protein lysates from the untreated DMS114, H1581, H1703 and H520 lung cancer cell lines. Taken together, each measurement group consisted of AZD4547-treated cells labelled with heavy SILAC, DMSO-treated cells labelled with medium SILAC and untreated light standard (Figure 47). 
A

\section{Standard light mixture}

1:1:1:1 Mixture of:

1. H1581 (responder cell line)

2. DMS114 (responder cell line)

3. $\mathrm{H} 520$ (resistant cell line)

4. H1703 (resistant cell line)
B

DMSO medium SILAC

1. H1581 responder

2. H520 non-responder

3. H1581 constitutively active Akt

4. H1581 induced resistance C9

5. H1581 induced resistance C11
C

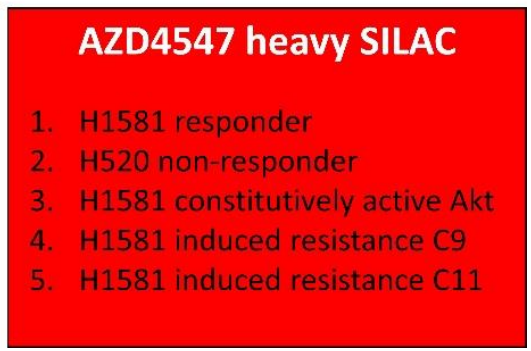

\section{Figure 47: Design of SILAC metabolic labeling of cell lines.}

Cell lines were divided into three groups: protein quantification standard cultured in light growth medium, DMSO-treated group cultured in medium SILAC growth medium and AZD4547-treated group cultured in heavy SILAC growth medium.

To ensure complete incorporation of heavy amino acids in treated cells, cell lines were incubated with either heavy or medium SILAC growth medium for at least ten cell divisions. Next, cells were harvested at confluency of $70-80 \%$. Cell pellets were divided into two groups as following: one group for global proteome (GPome) and the second group for tyrosine phosphopeptides enrichment (pYome). Global proteome cell pellets were lysed with NP-40 lysis buffer, while pYome cell pellets were lysed with urea lysis buffer. All samples were collected for two biological replicates of each experiment. Enrichment of the low-abundant phosphopeptides required starting with large amounts of protein lysates. I collected a total of $250 \mathrm{mg}$ of the light standard cell lysates, $195 \mathrm{mg}$ of the medium SILAC labeled cell lysates and $195 \mathrm{mg}$ of the heavy SILAC labeled cell lysates (Figure 48).

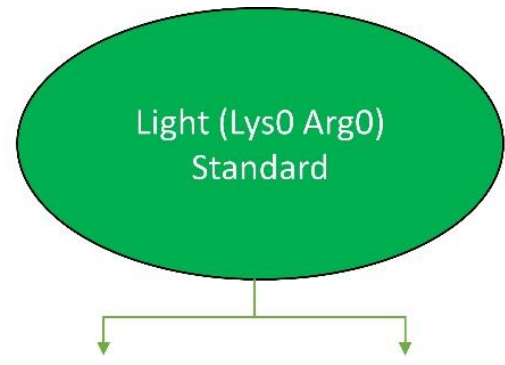

pYome

GPome

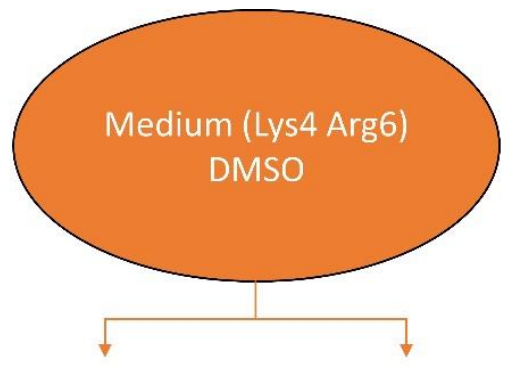

pYome

GPome

$10-20 \mathrm{mg} /$ cell line $5-10 \mathrm{mg} /$ cell line $10 \mathrm{mg} /$ cell line $5 \mathrm{mg} /$ cell line

2 biological replicates $250 \mathrm{mg}$
2 biological replicates $195 \mathrm{mg}$

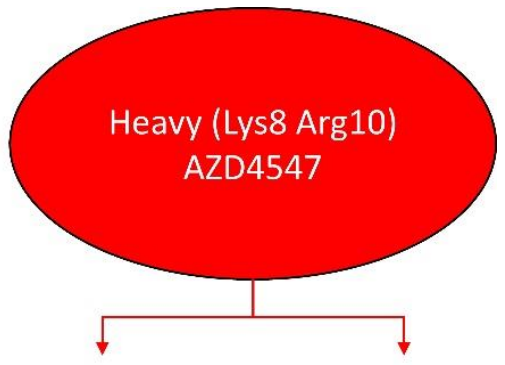

pYome GPome $10 \mathrm{mg} /$ cell line $5 \mathrm{mg} /$ cell line

2 biological replicates $195 \mathrm{mg}$

Figure 48: Protein lysates assembly for mass spectrometric analysis. 
Protein lysates were divided into two groups; the first group was for global proteome phosphorylated proteins and the second groups was enriched for tyrosine phosphorylated proteins.

\subsubsection{Phosphoproteomic LC-MS/MS data quality control}

Phosphopeptides-enrichment and fractionation was done in collaboration with group of Prof. Dr. Thomas Oellerich at department of Hematology and Oncology, University Hospital of Frankfurt, Germany. Peptides sequencing was done in collaboration with the group of Prof. Dr. Henning Urlaub at Max Planck institute for biophysical chemistry, Goettingen, Germany. For each sample, the three groups of light standard, medium and heavy labelled protein content were mixed in ratios of 1:1:1 then digested using trypsin. Titanium dioxide (TiO2) and phosphotyrosine antibodies were used to enrich global and tyrosine phosphorylated peptides, respectively. Afterwards, enriched phosphopeptides were fractionized using strong cation exchange (SCX) then applied on HPLC system coupled online to mass spectrometer.

Raw data out of the mass spectrometer were analyzed through MaxQuant bioinformatics tool, while I used Perseus tool to perform the quality control and downstream differential analysis of the data. After filtering reverse and potential contaminant values, numeric Venn diagrams showed a total of 14014 quantified phosphosites in the global proteome group and a total of 831 quantified sites in the tyrosine enriched group (Figure 49).

A

GPome quantified phosphosites (total=14014)

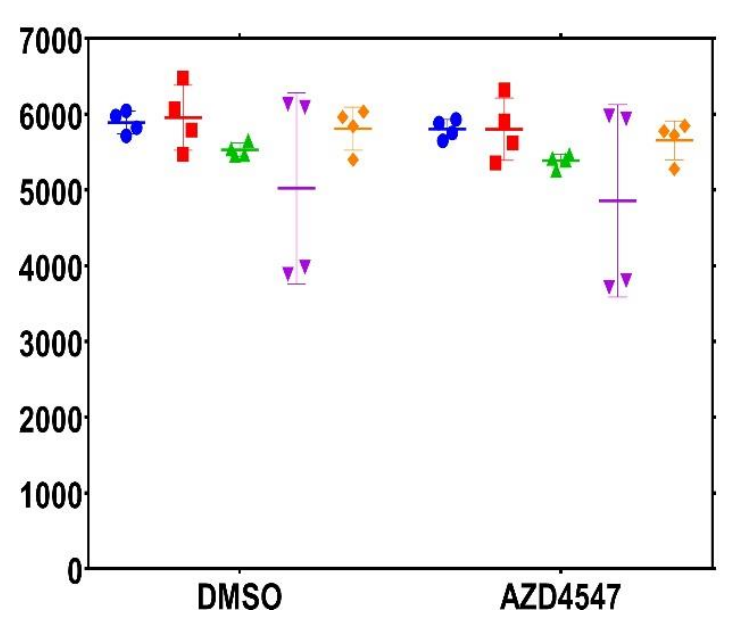

B

pYome quantified phosphosites (total=831)

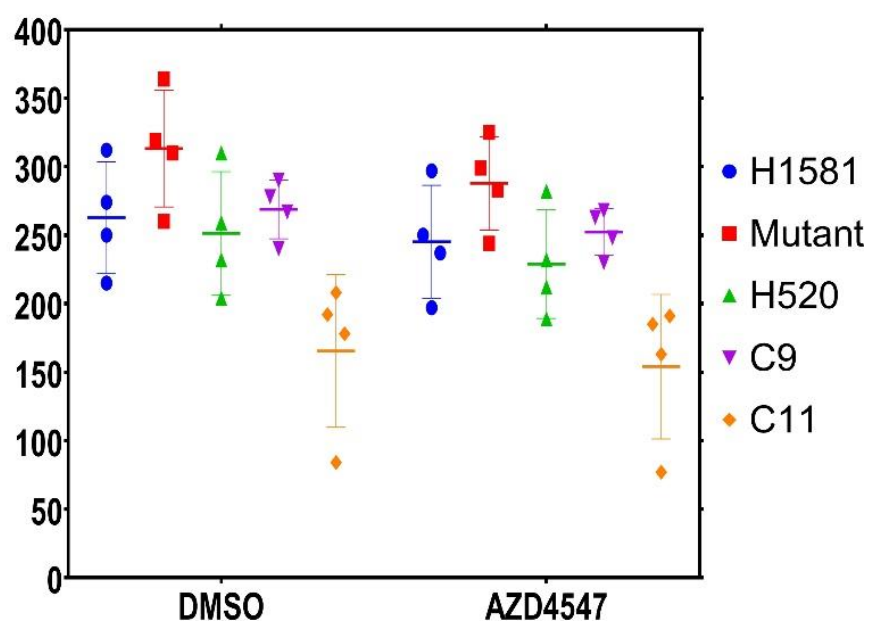

Figure 49: Numeric Venn diagram showed total number of quantified phosphosites among different groups. Phosphosites were quantified among two biological replicates and two technical replicates in each of the groups. 
With the purpose of assessing the quality of the replicates among different quantified groups, I used multiple scatter plot assigned with regression values (R), which showed similarities between different technical and biological? replicates in the GPome and pYome groups (Figures 50 and 51).

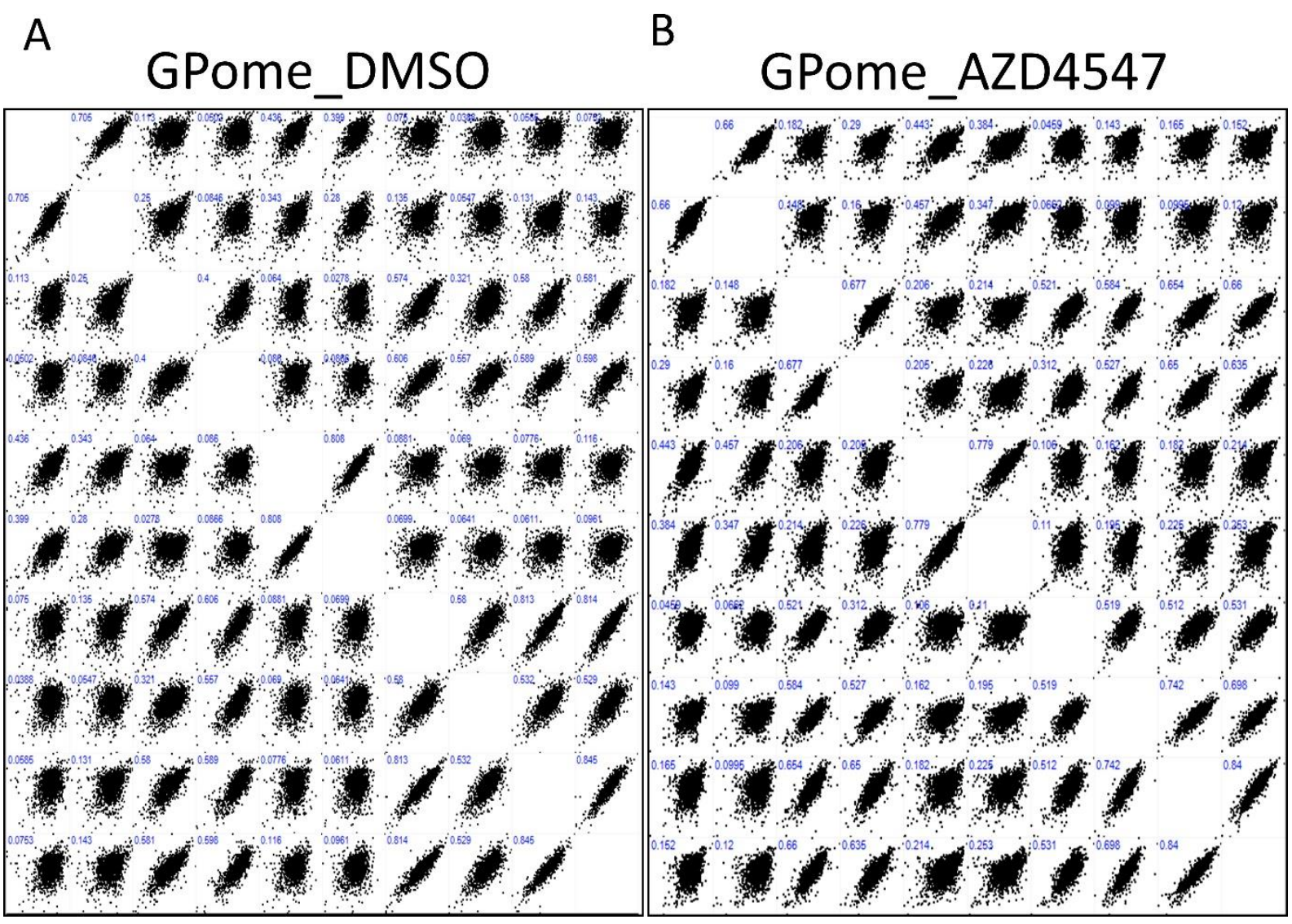

Figure 50: Multiple scatter plot for all samples in global proteome phosphopeptides group.

Quality of different replicates among global proteome phosphopeptides group was assessed through testing similarities between biological and technical replicates using regression number $R$ in DMSO treated (A) and AZD4547 treated (B) cells. 


\section{A pYome_DMSO B pYome_AZD4547}

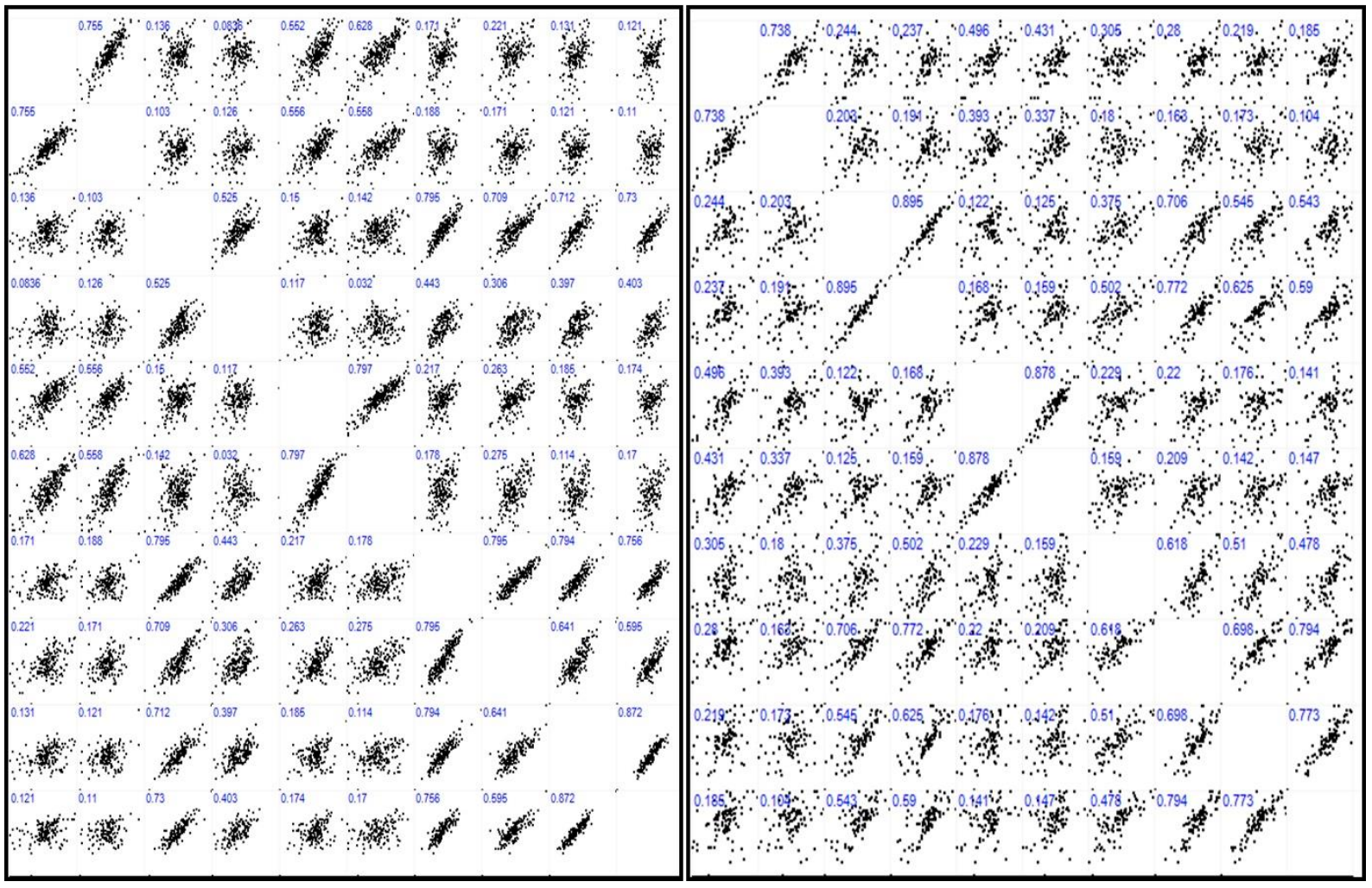

Figure 51: Multiple scatter plot for all samples in phospho-tyrosine enriched group.

Regression values $(R)$ showed quality of technical and biological replicates among tyrosine enriched phosphopeptides group in control group (A) and AZD4547 treated group (B).

Thereafter, I tested the normal distribution of values among all technical and biological replicates in all the groups (Figures 52 and 53). Histograms showed normal distribution of the values, which enabled the progress to the next step in the analysis. 


\section{A}

GPome_DMSO

\section{B GPome_AZD4547}

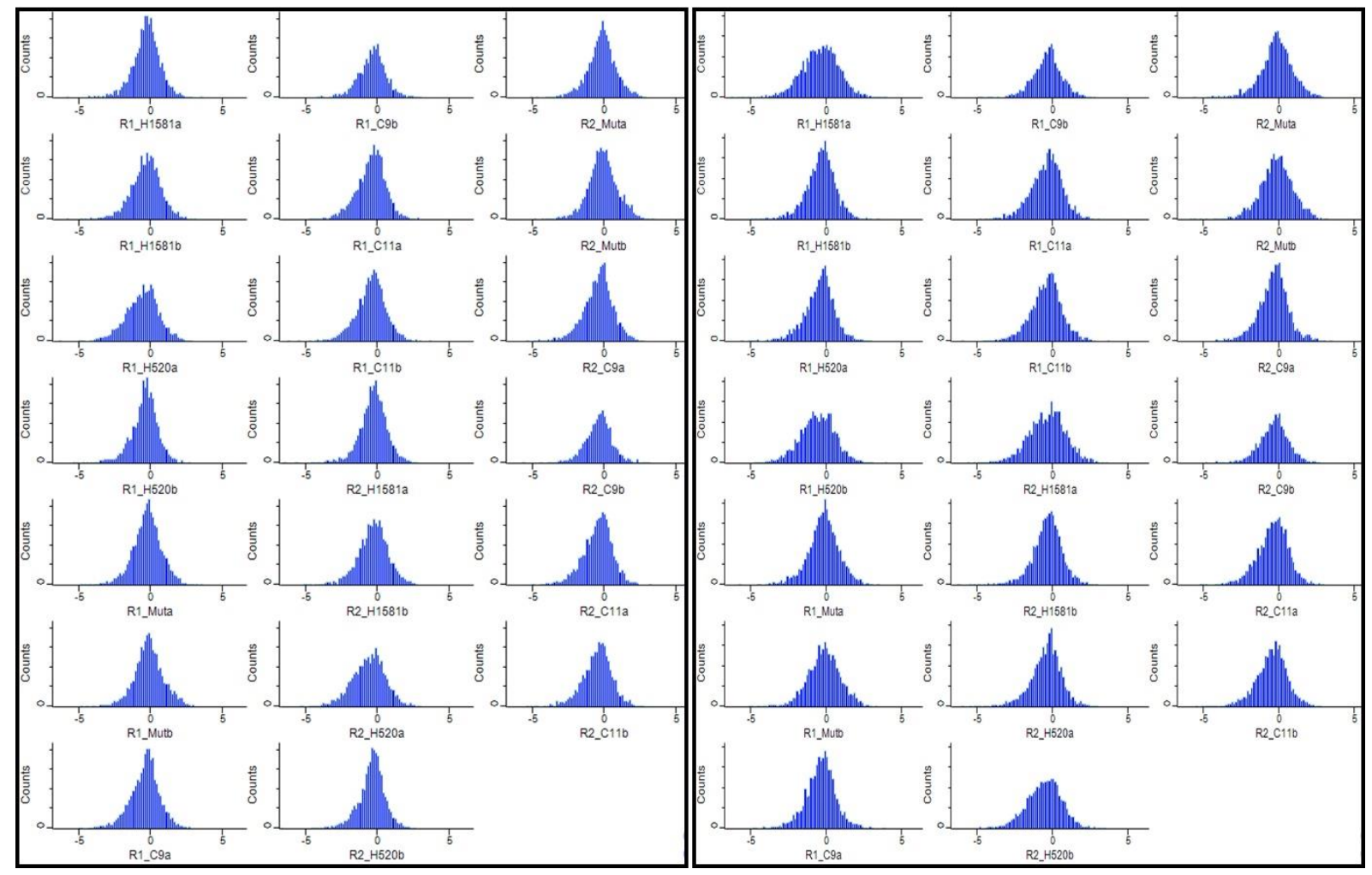

Figure 52: Normal distribution of values in global proteome group.

Histograms showed normal distribution of all values among all the technical and biological replicates in DMSO treated cells (A) and AZD4547 treated cells (B) of the GPome group. 
A pYome_DMSO

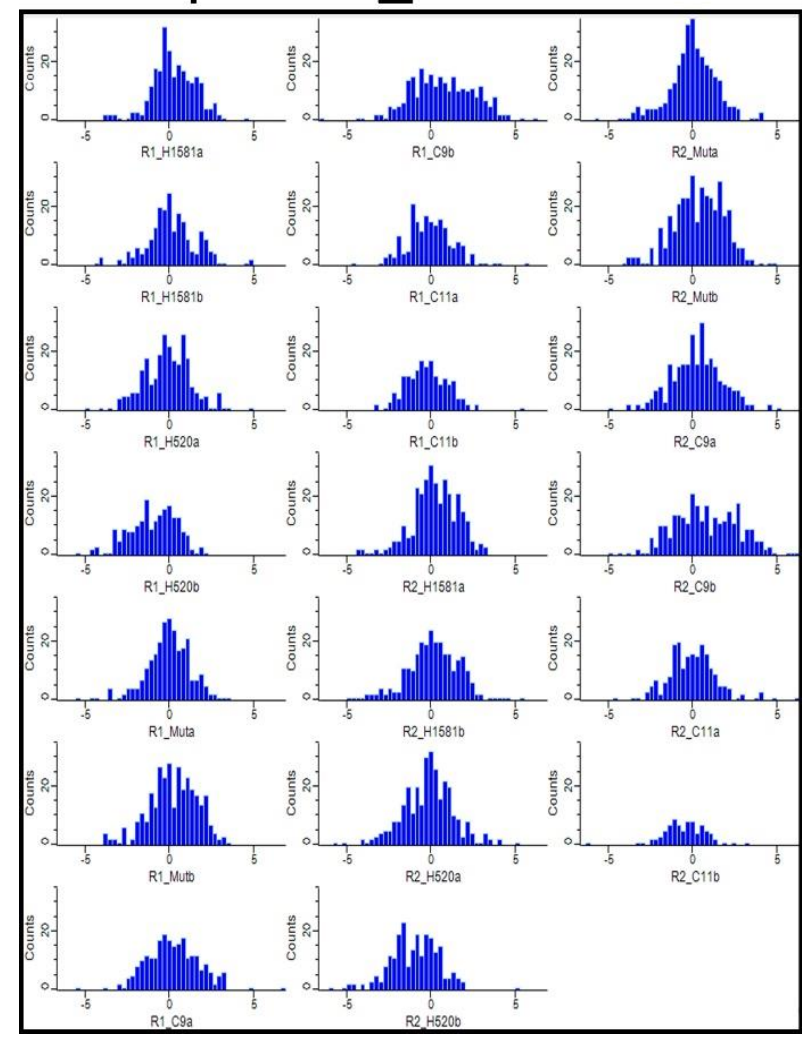

B pYome_AZD4547

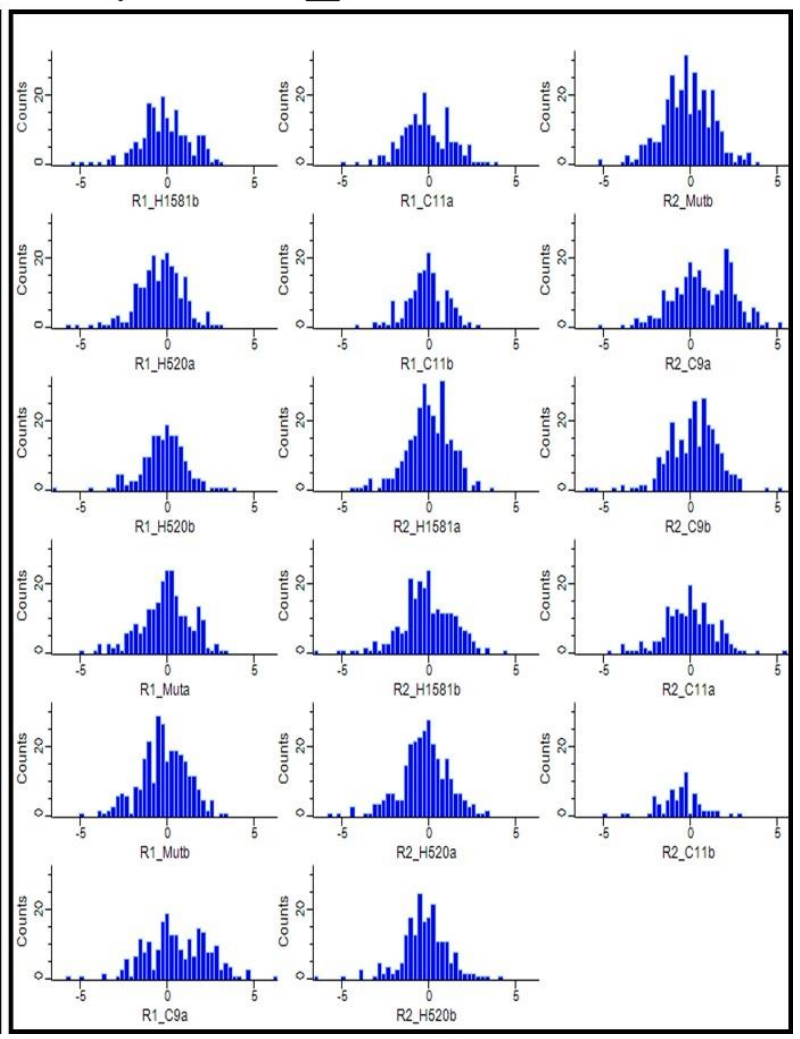

Figure 53: Normal distribution of values in phospho-tyrosine enriched group.

Histograms showed the normality in distribution in technical and biological replicates of DMSO treated cells (A) and AZD4547 treated cells (B) in pYome group.

\subsubsection{Phosphoproteomic LC-MS/MS data bioinformatics functional analysis}

In order to perform statistical analysis tests, I calculated the average of technical replicates values and assigned the biological replicates in groups. Five groups where assigned as following: 1- H1581 (control group), 2- H1581 mutantAkt, 3- H520 (native resistant), 4- C9 (induced resistant) and 5-C11 (induced resistant). Sites were filtered for localization probability of more than 0.75 and $70 \%$ valid values. I performed multiple sample ANOVA test to compare all biological replicates among all groups together. The SO parameter was set to zero, permutation-based FDR was chosen with FDR value of 0.05 and number of randomizations was set to 250 .

In total, 731 and 667 phosphosites were significantly regulated among the global proteome in DMSO and AZD4547 treated cells, respectively. Heatmaps of enriched significant phosphosites showed clustering between parental H1581 and H1581 mutant-Akt on one side and between native resistant $\mathrm{H} 520$ and induced resistant $\mathrm{C} 9$ and $\mathrm{C} 11$ cells on the other side (Figure 54). When phosphosites were clustered into 
three groups, the groups appeared to be as following: $\mathrm{H} 1581$ as first cluster, $\mathrm{H} 1581$ mutantAkt as second cluster and H520, C9 and C11 as third cluster. In pYome groups, 34 and 86 phosphosites were significantly regulated among all groups in DMSO and AZD4547 treated cells, respectively. ANOVA significant enriched heatmaps showed again two main clusters. The first cluster consisted of H1581 control and mutant-Akt cells. The second cluster consisted of H520, C9 and C11 cells (Figure 55).

A

GPome_DMSO

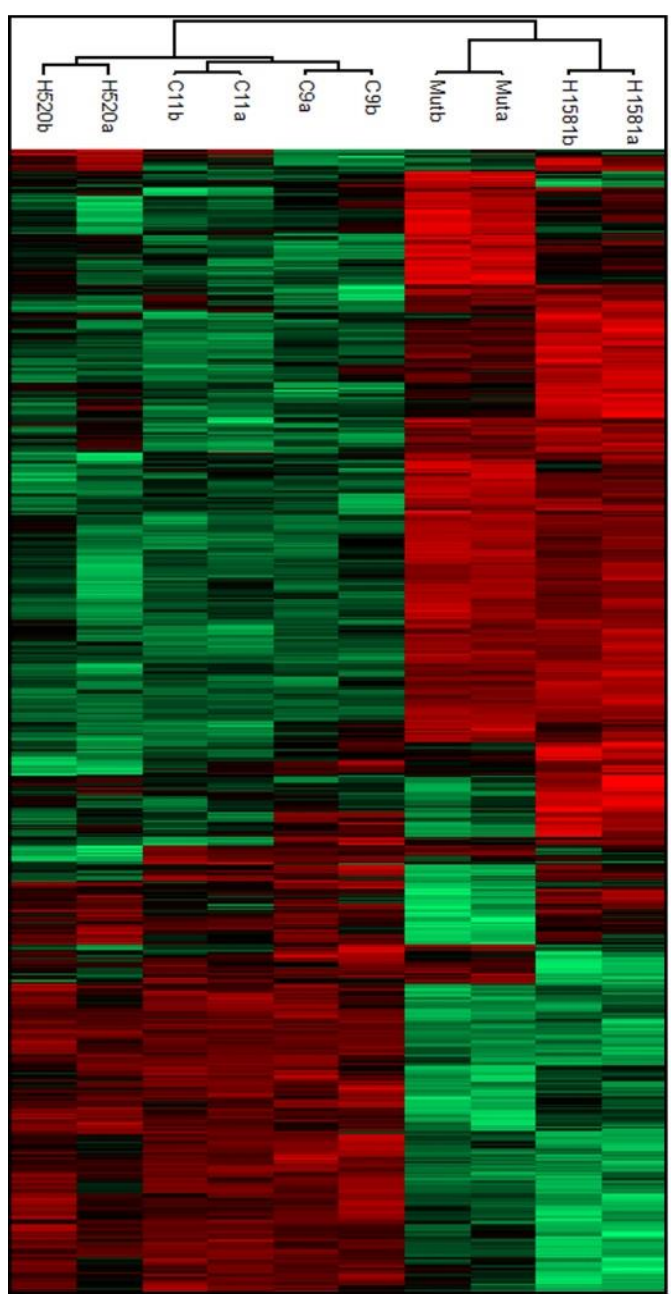

B
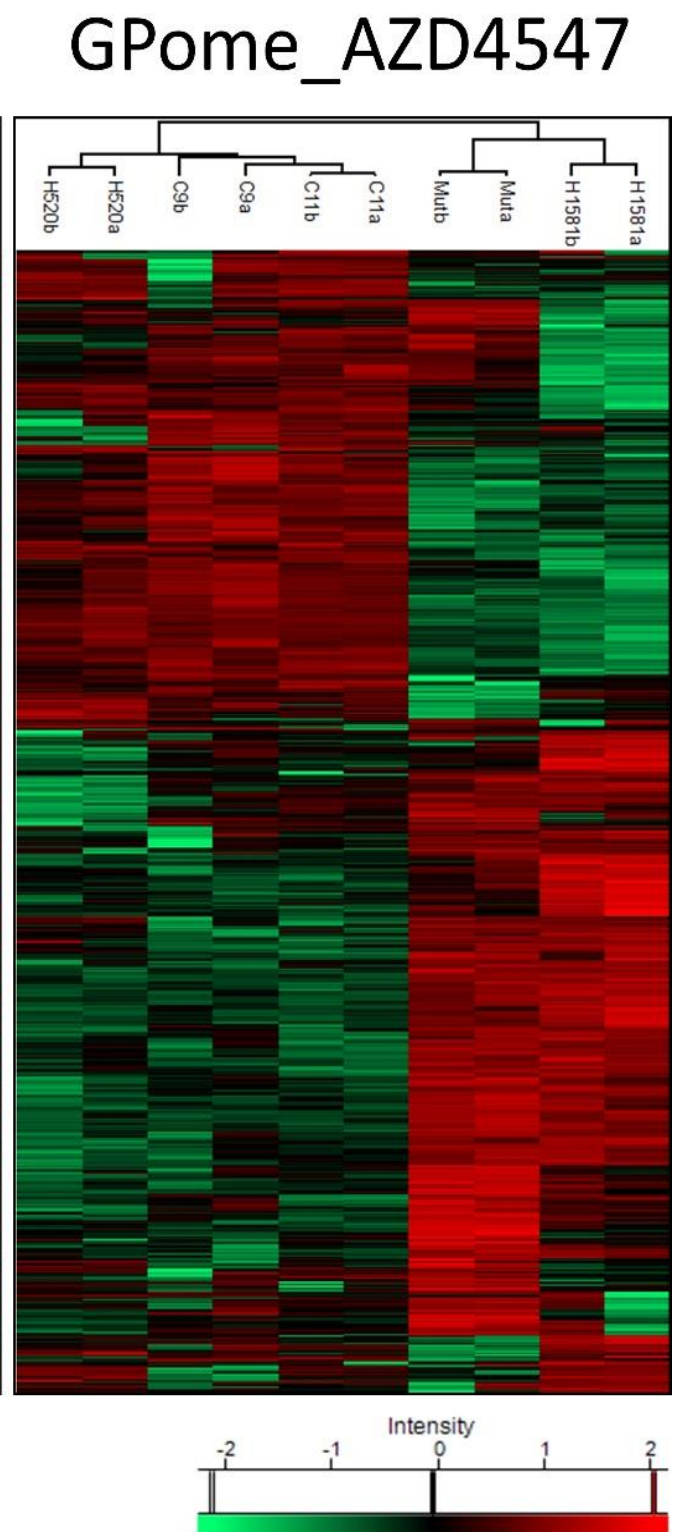

Figure 54: Heatmaps of significantly differentiated intensities of phosphosites among different GPome samples.

Intensities of phosphosites were analyzed using ANOVA multiple test with FDR value of 0.05 in DMSO treated (A) and AZD4547 treated (B) cells of global proteome samples. Intensities were z-normalized and imputated for missing values. 
A

pYome_DMSO

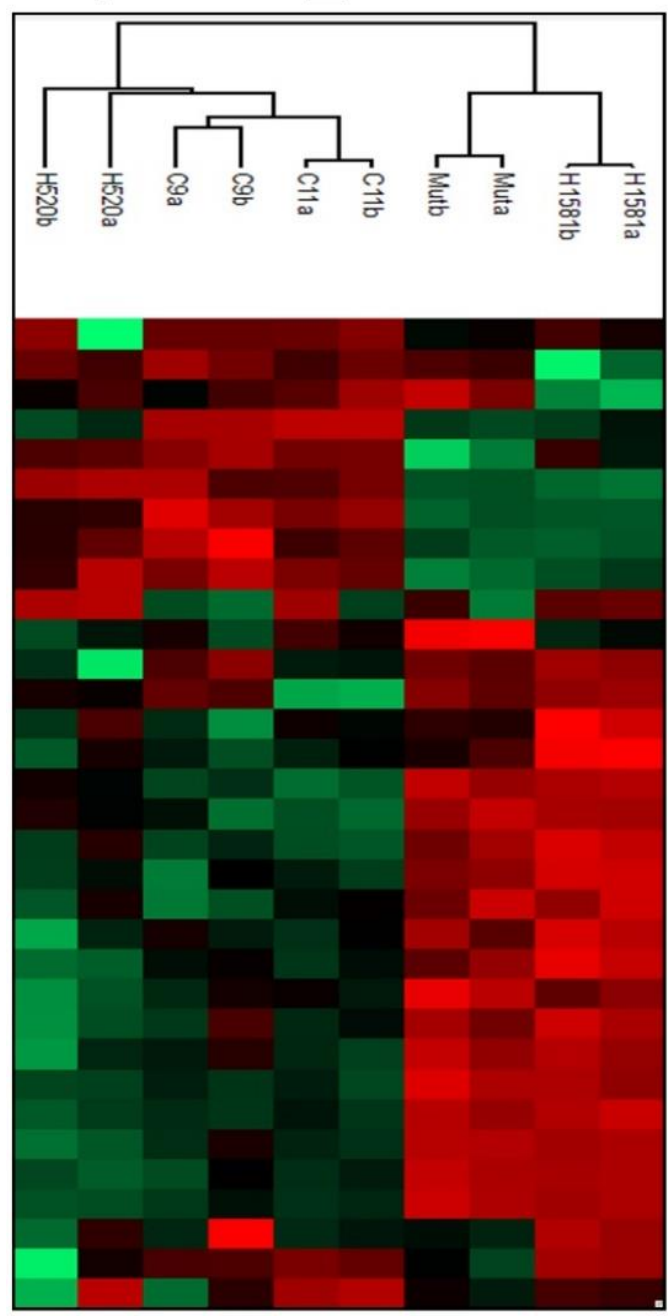

B

\section{pYome_AZD4547}
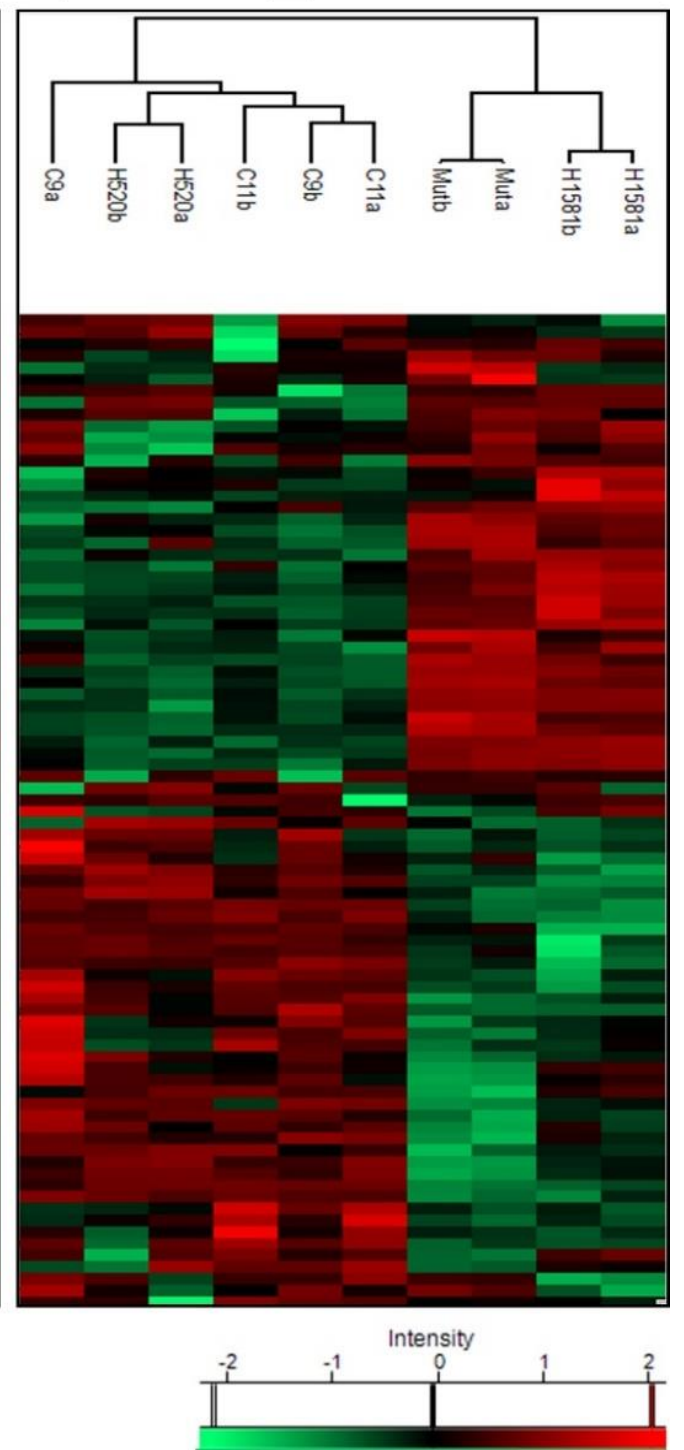

Figure 55: Heatmaps of significantly differentiated intensities of phosphosites among different pYome samples.

Intensities of phosphosites were analyzed using ANOVA multiple test with FDR value of 0.05 in DMSO treated (A) and AZD4547 treated (B) cells of tyrosine enriched phosphopeptides. Intensities were znormalized and imputated for missing values.

Next, I compared intensities of phosphosites in each sample on its own to the control $\mathrm{H} 1581$ cell line. To achieve this, I used volcano plots and t-test with an FDR value of 0.05 , S0 of 0.1 and number of randomizations of 250. In the GPome analysis, the number of significantly differentially phosphorylated sites were 531, 293, 553 and 1581 in the groups of mutantAkt, H520, C9 and C11, respectively. In the pYome analysis, number of significantly differentially phosphorylated sites were 11, 34, 47 
and 75 in the groups of mutantAkt, H520, C9 and C11, respectively. (Figures 56 and 57).

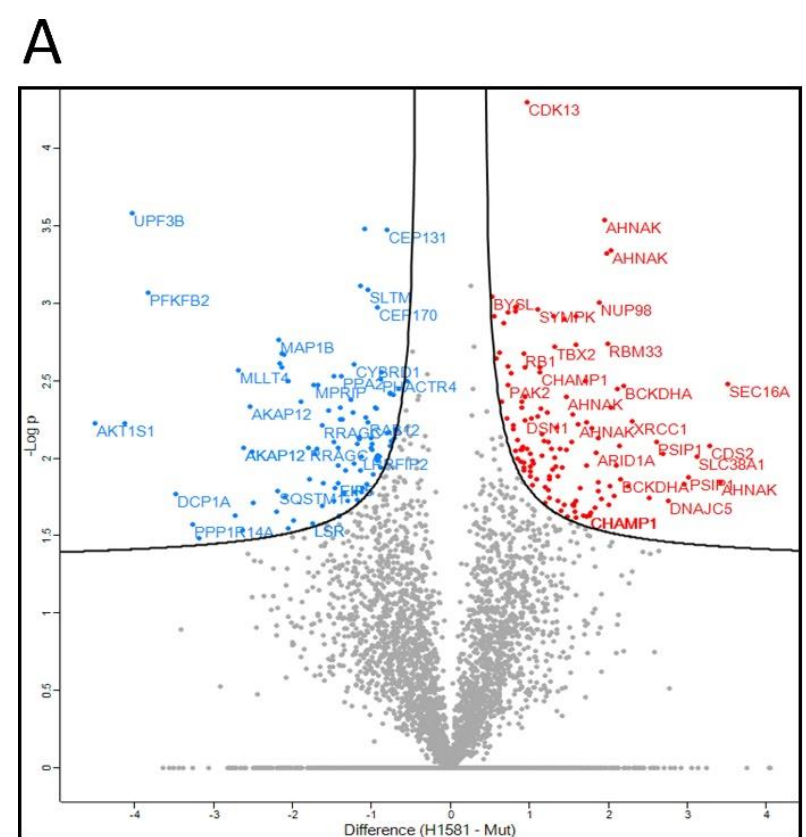

B
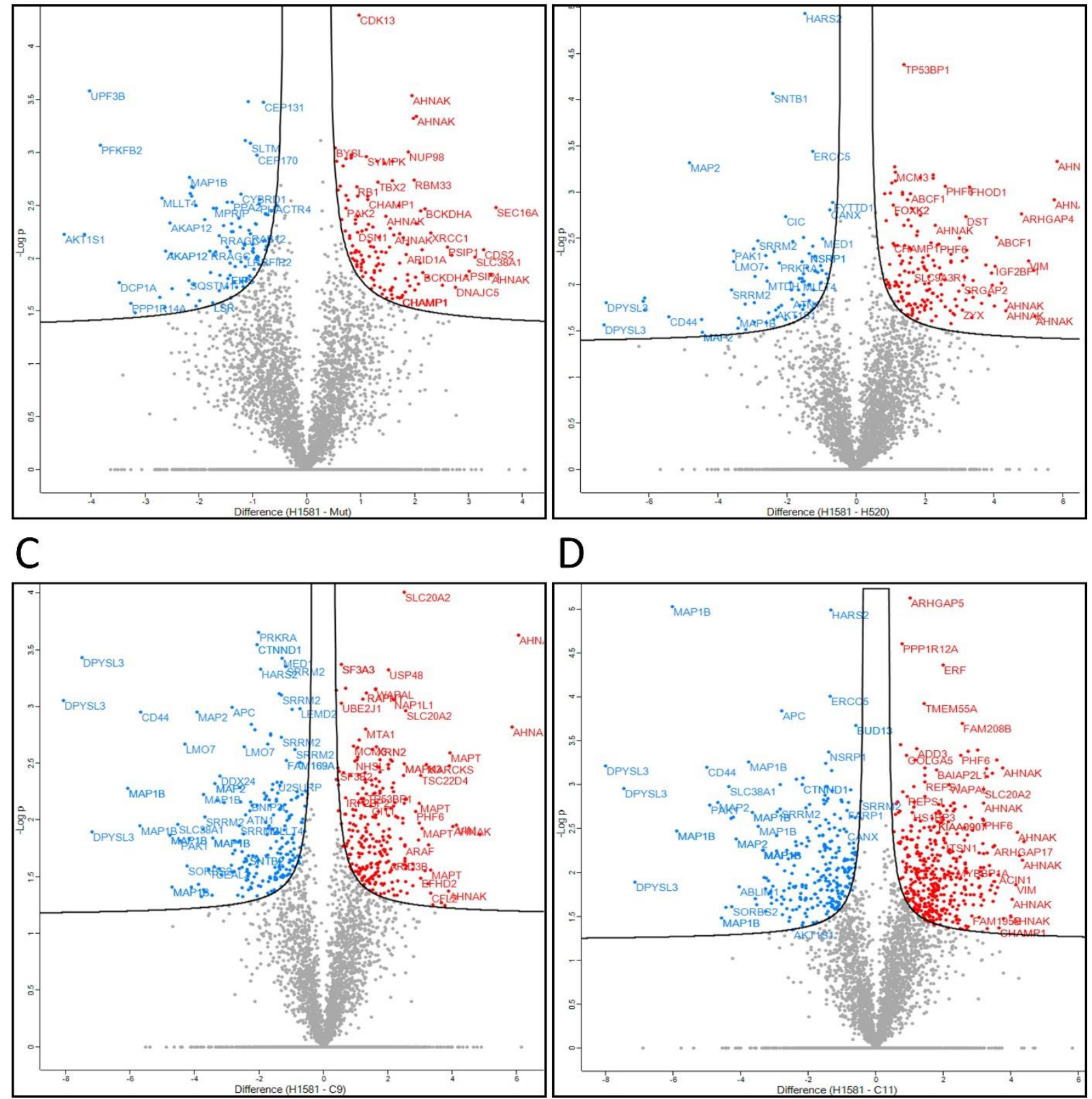

D

Figure 56: Volcano plots show significantly differentiated phosphosites.

Intensities of GPome phosphosites in the control $\mathrm{H} 1581$ cell line were compared to each of the $\mathrm{H} 1581$ mutantAkt (A), H520 (B), C9 (C) and C11 (D) DMSO-treated cells. Figures show t-test with an FDR of 0.05 and $\mathrm{S} 0$ of 0.1 . 
A

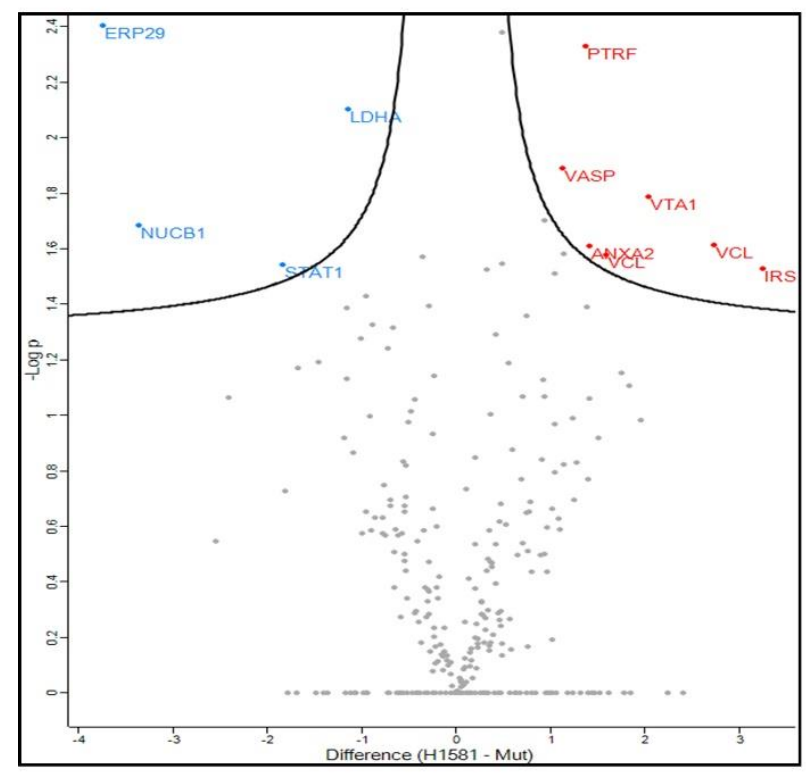

B

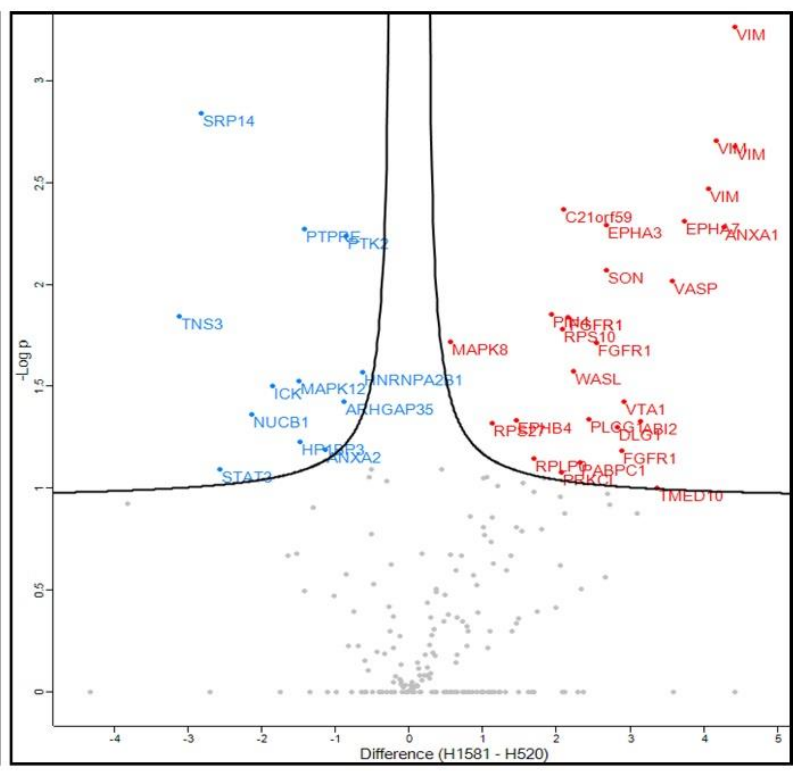

C

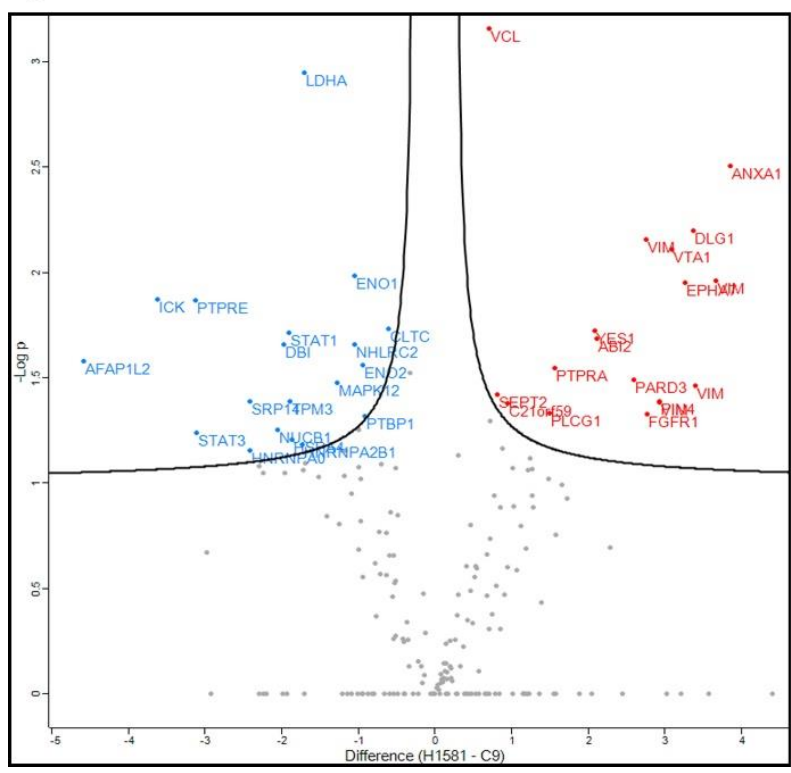

D

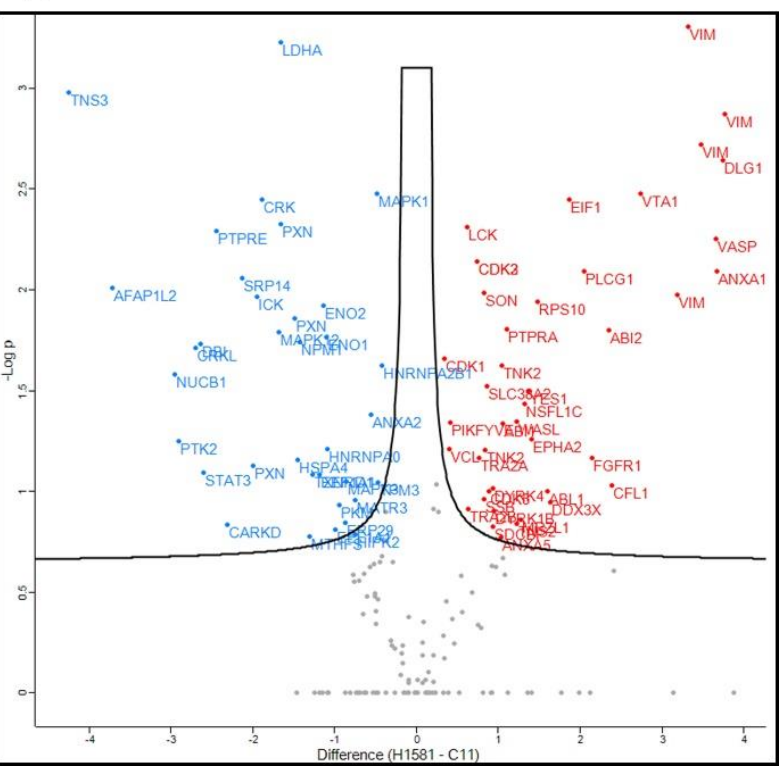

Figure 57: Volcano plots of significantly differentiated phosphosites in pYome samples.

Intensities of tyrosine enriched phosphosites in the control H1581 cell line were compared to each of the H1581 mutantAkt (A), H520 (B), C9 (C) and C11 (D) DMSO-treated cell lines. Figures show t-test with an FDR of 0.05 and $\mathrm{S} 0$ of 0.1 .

In order to investigate targets activation that could have led to FGFR1 inhibition resistance, I examined the lists of t-test and ANOVA significant sites among all the groups. Examination of the phosphosites depended on three main factors. The first factor is the clustering of phosphosites into H1581 sensitive parental cell line, H1581 mutantAkt (downstream Akt resistance) and native and induced resistant H520, C9 
and $\mathrm{C} 11$ cell lines. The second factor is the functional annotation of each of the sites using DAVID, String and Gene cards databases. The last factor is the literature review where I checked for biological relevance of each of the sites as well as the interaction of these sites together. Using these three factors, I discovered a strongly significantly regulated pathway upstream of Akt, which is upregulated in the native and induced resistant cells but not in the mutant resistant cell line. The pathway consisted mainly of CD44, FAK, PAK1, Paxillin, RAC1, CDC42 and STAT3 proteins. At the same time, Akt and its downstream target, PRAS40, were significantly highly activated in all resistant cell lines compared to parental H1581 control (Figure 58). Further sites, which were significantly higher or lower activated in resistant cell lines compared to parental $\mathrm{H} 1581$ control can be connected to processes in the body like apoptosis inhibition, proliferation or survival promotion and metastasis induction (Figure 59). As a control, I demonstrated the efficacy of FGFR1 signaling inhibition via AZD4547 through the reduction of ERK inhibition at Y187 phosphosite (Figure 58).

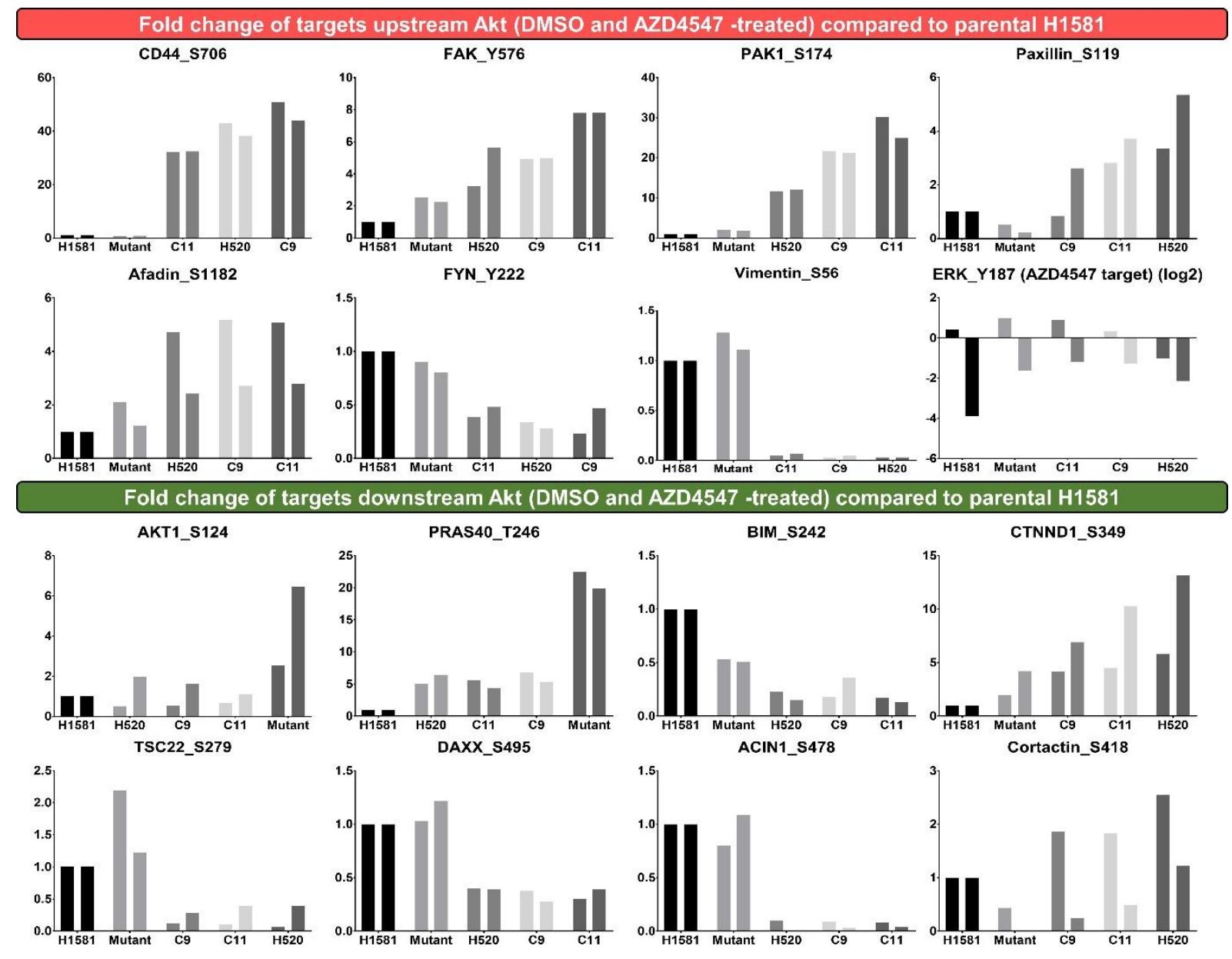

Figure 58: Fold change of significantly over and down phosphorylated targets upstream and downstream Akt in resistant cell lines compared to control H1581 cell line. 
ANOVA significant targets are shown as fold change of the parental $\mathrm{H} 1581$ control cell line in the DMSO and AZD4547 treated conditions. ERK phosphotyrosine site at Y187 is shown as log2 value to demonstrate the efficacy of AZD4547 inhibition.

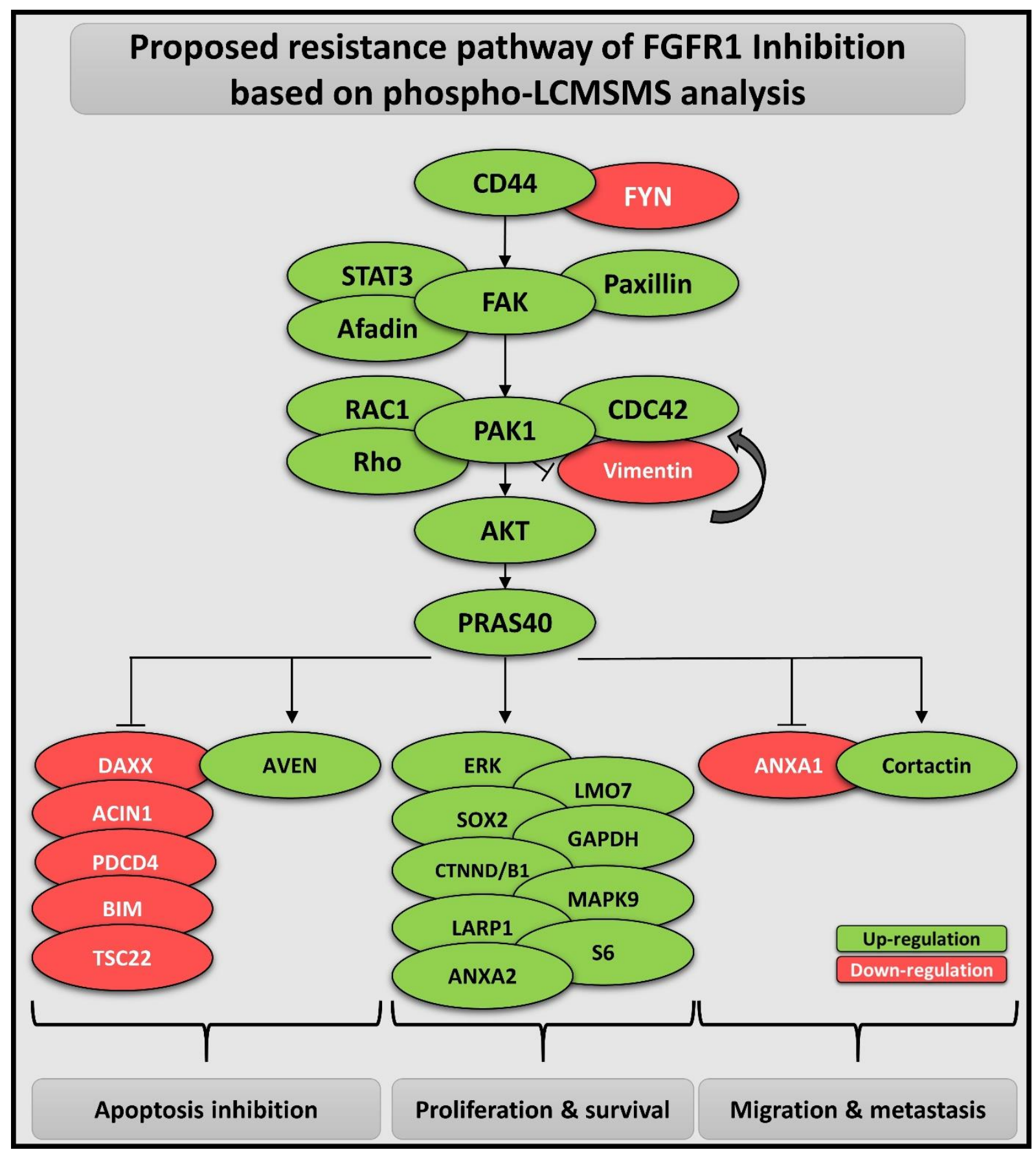

Figure 59: Proposed resistance pathway in FGFR1 inhibition resistant cell lines compared to H1581 control cell lines.

Effectors upstream of Akt were significantly differentiated in all resistant cells except of H1581 mutantAkt cell line, while effectors downstream of Akt were significant regulated in all resistant cells compared to sensitive control. 


\subsubsection{Functional validation of the proposed FGFR1 inhibition resistance pathway}

In an effort to functionally validate the new molecular targets in the resistance pathway, I treated the native resistant cell lines $\mathrm{H} 520$ and $\mathrm{H} 1703$ and the induced resistant clones $\mathrm{C} 4, \mathrm{C} 9$ and $\mathrm{C} 11$ with PAK1 or FAK inhibitors alongside with the FGFR1 inhibitor. Two highly specific PAK1 inhibitors were used. FRAX597 is an ATP competitive inhibitor of PAK1 with an IC50 value of $8 \mathrm{nM}$ as reported by the provider (Selleck chemicals), while IPA-3 is a non-ATP competitive PAK1 inhibitor with an IC50 value of $2.5 \mu \mathrm{M}$ as reported by the provider (Selleck chemicals). A single potent FAK inhibitor was used. PF562271 is a potent ATP competitive inhibitor of FAK with An IC50 of $1.5 \mathrm{nM}$ as reported by the supplier (Selleck chemicals).

AZD4547 was used as an FGFR1 inhibitor. The five cell lines were treated with elevated concentrations of either one of the inhibitors or both combined. Results of the combination therapy were measured through MTS colorimetric assay (Figures 60 and 61). Nature of interaction between FGFR1 and PAK1 or FAK inhibitors was assessed through Chou-Talalay combination index $(\mathrm{Cl})$ across the cell lines. $\mathrm{Cl}$ index showed strong synergistic effect between AZD4547 and each of FRAX579, IPA-3 and PF562271 inhibitors in all of the tested cells (Figure 62). 
A FGFR1 inhibition, AZD4547 ( $\mu$ M)

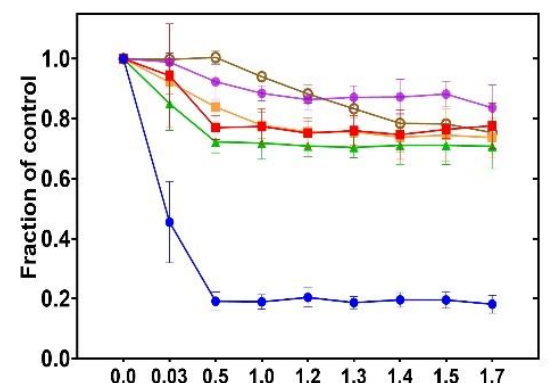

$\rightarrow \mathrm{H} 1703$

$-\mathrm{H} 520$

- Clone 4R

- Clone 9R

$\rightarrow$ Clone $11 \mathrm{R}$

$\rightarrow$ H1581 Parental

$\mathrm{C}$

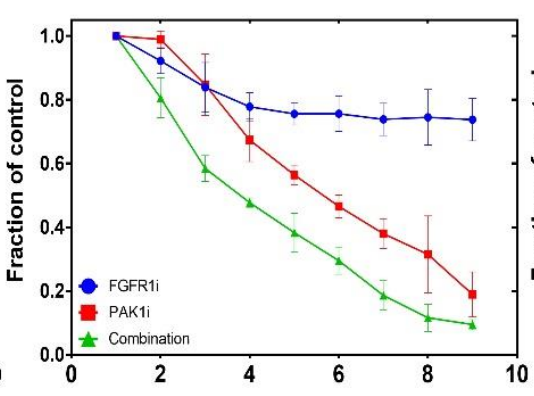

$\mathrm{F}$

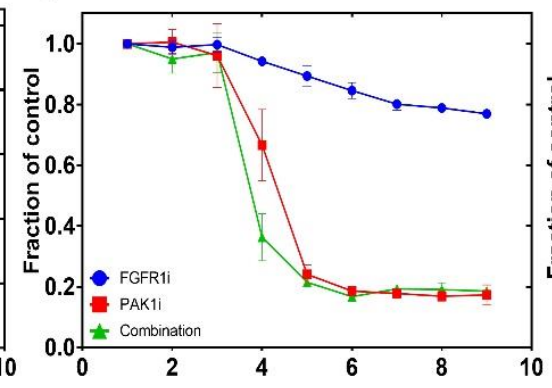

H1703- IPA3

$\begin{array}{clllllllll}\begin{array}{c}\text { Inhibitor } \\ (\mu \mathrm{M})\end{array} & 1 & 2 & 3 & 4 & 5 & 6 & 7 & 8 & 9\end{array}$

$\begin{array}{lllllllll}\text { FGFR1 DMSO } & 0.03 & 0.50 & 1.00 & 1.20 & 1.30 & 1.40 & 1.50 & 1.70\end{array}$

$\begin{array}{lllllllll}\text { FRAX579 DMSO } & 0.50 & 0.75 & 1.00 & 1.20 & 1.25 & 1.30 & 1.40 & 1.50\end{array}$

IPA3 DMSO 2.5010 .0015 .0020 .0025 .0030 .0035 .0040 .00

$\begin{array}{lllllllll}\text { PF562271 DMSO } & 0.01 & 0.10 & 0.50 & 1.00 & 2.00 & 3.00 & 4.00 & 5.00\end{array}$

H520-IPA3 D H520-PF562271
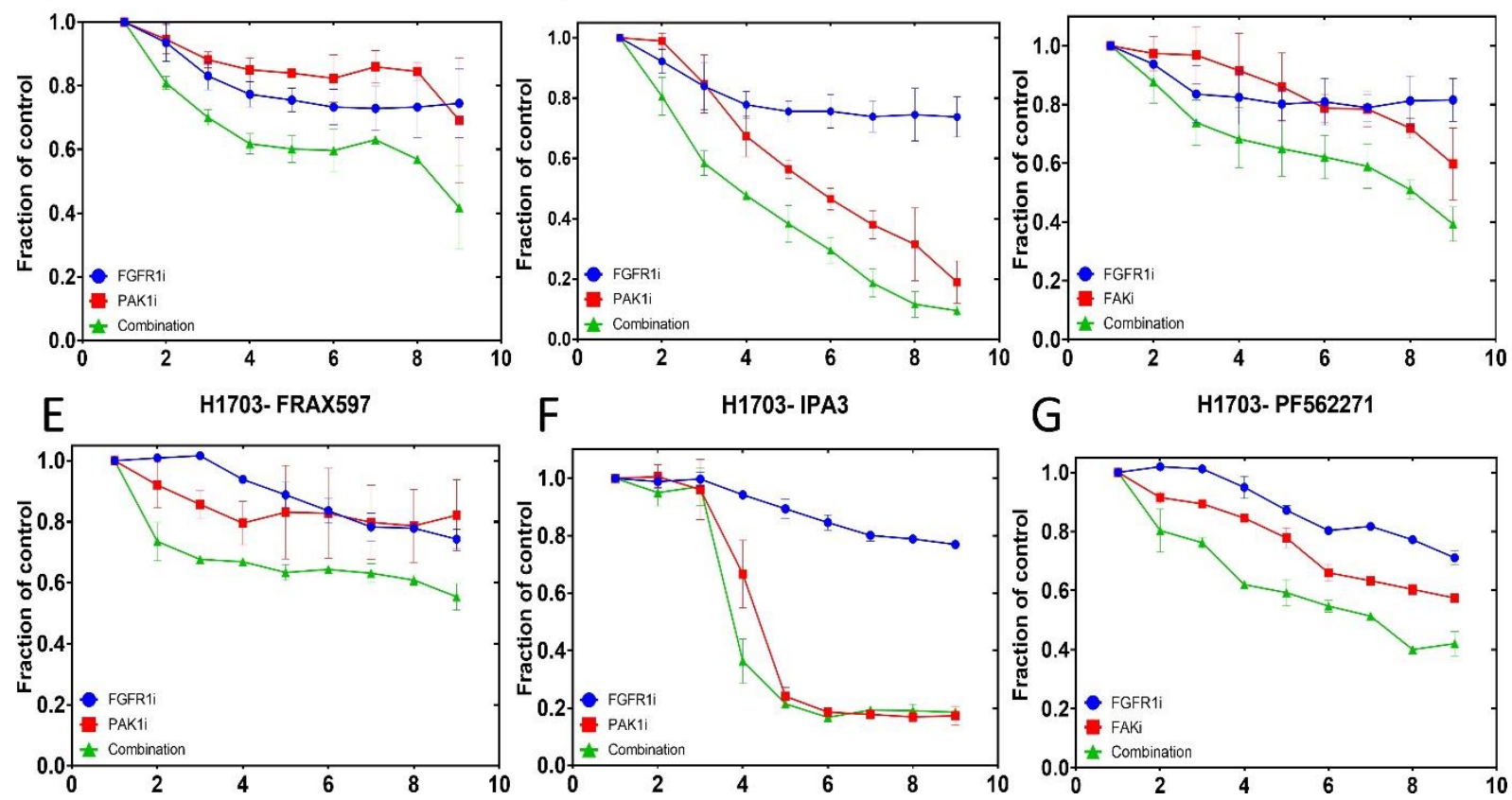

G

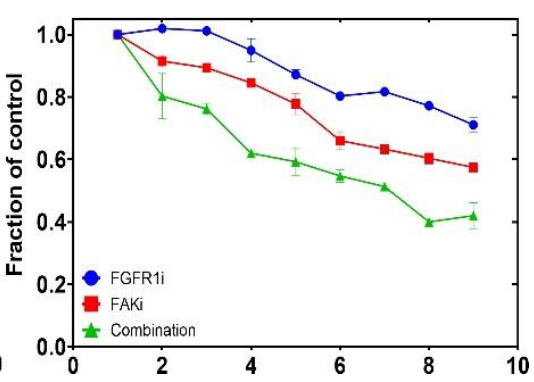

Figure 60: MTS assays show combination between PAK1 or FAK and FGFR1 inhibition in FGFR1 inhibition intrinsic resistant cell lines.

Assay shows resistance to the FGFR1 inhibitor AZD4547 in all resistant cells (A). Combination inhibition between FGFR1 inhibitor (AZD4547) and PAK1 inhibitor (FRAX597) (B and E), PAK1 inhibitor (IPA3) (C and F) and FAK inhibitor (PF562271) (D and $G$ ) in intrinsic resistant cell lines. Inhibitors were used at the concentrations denoted in the table above. All experiments were conducted in three replicates. 
A

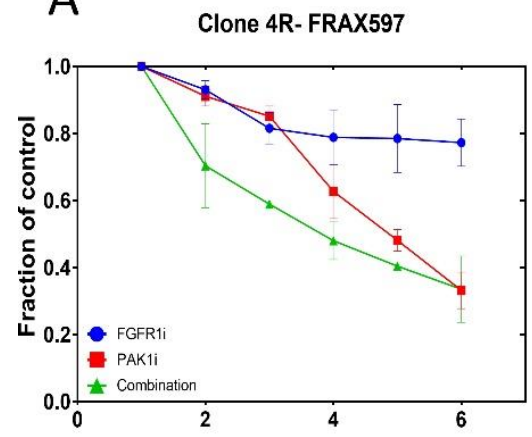

D

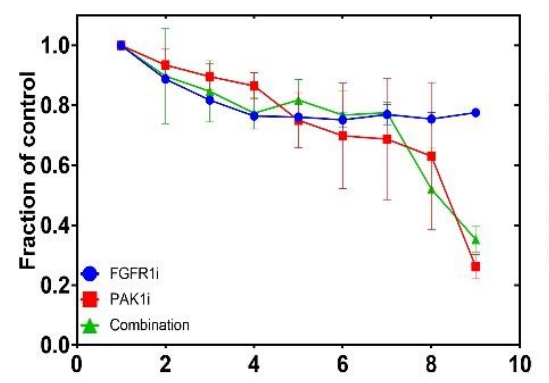

$\mathrm{G} \quad$ Clone 11R- FRAX597

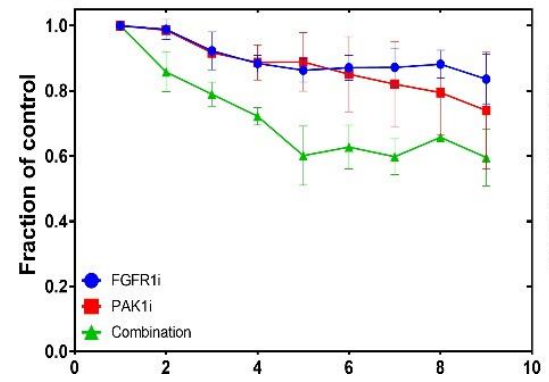

B

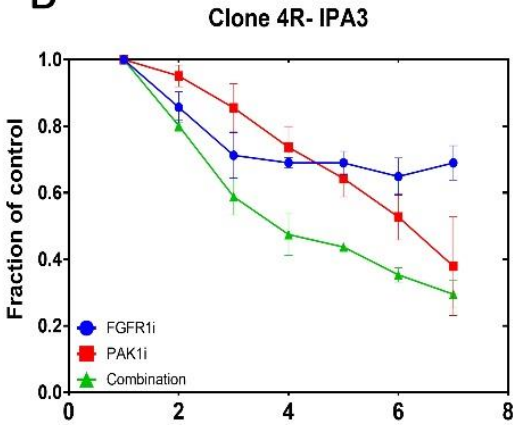

E

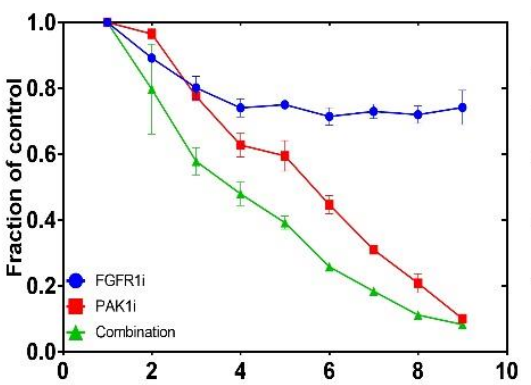

$\mathrm{H}$

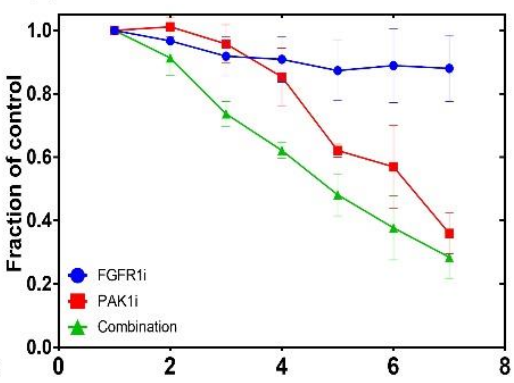

C

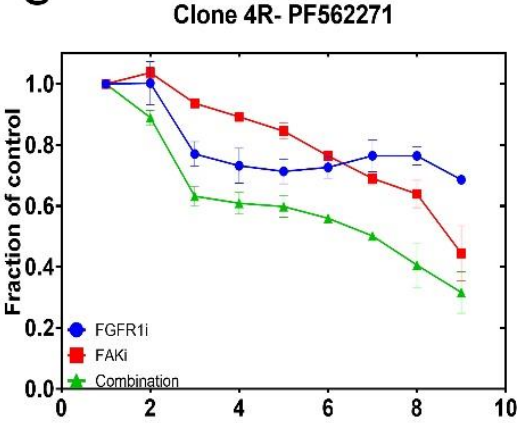

$\mathrm{F}$

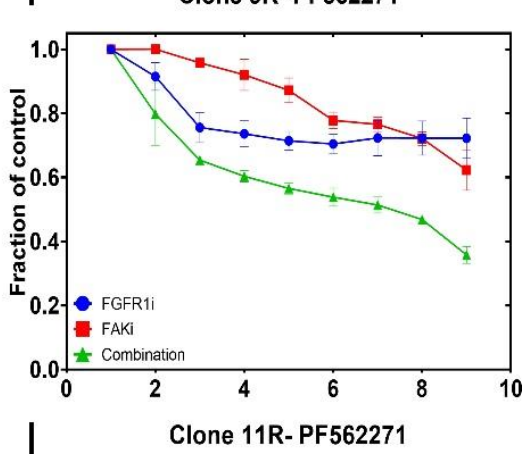

I

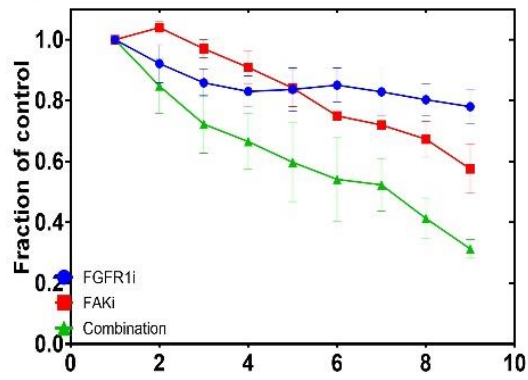

Figure 61 MTS assays show combination between PAK1 or FAK and FGFR1 inhibition in FGFR1 inhibition induced resistant cell lines.

Combination inhibition between FGFR1 inhibitor (AZD4547) and PAK1 inhibitor (FRAX597) (A, D and G), PAK1 inhibitor (IPA3) (B, E and H) and FAK inhibitor (PF562271) (C, F and I) in induced resistant clones. Inhibitors were used at the concentrations denoted in figure 60. All experiments were conducted in three replicates. 

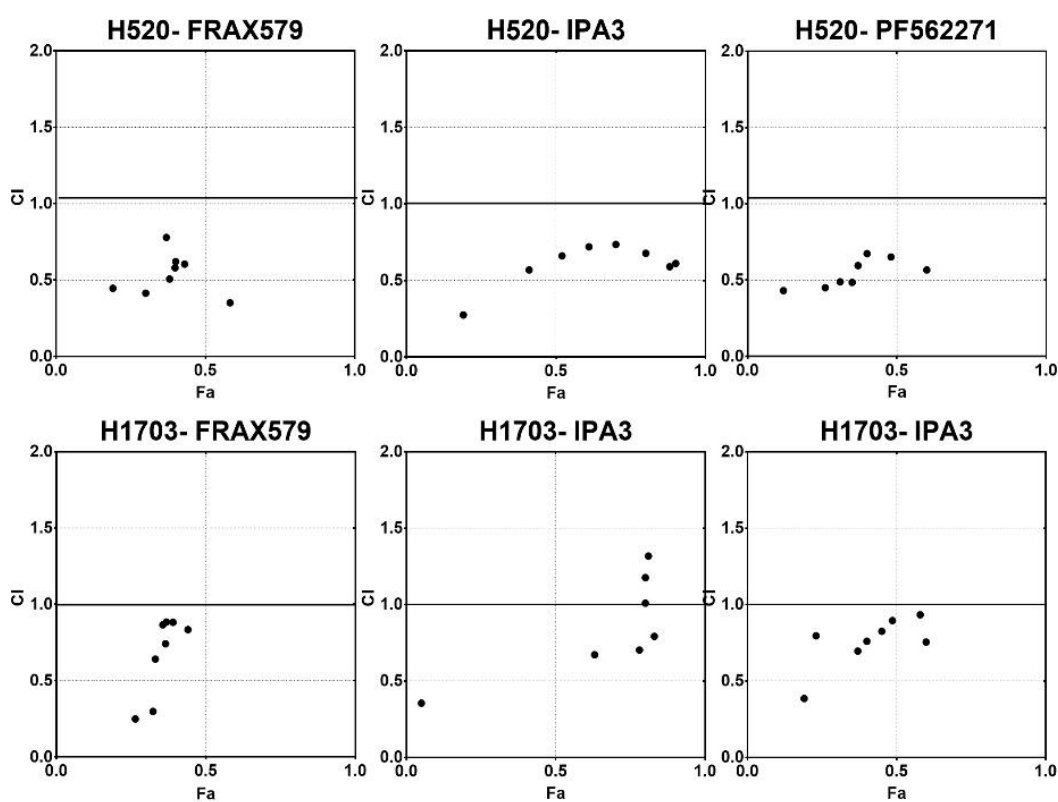

H1703- IPA3
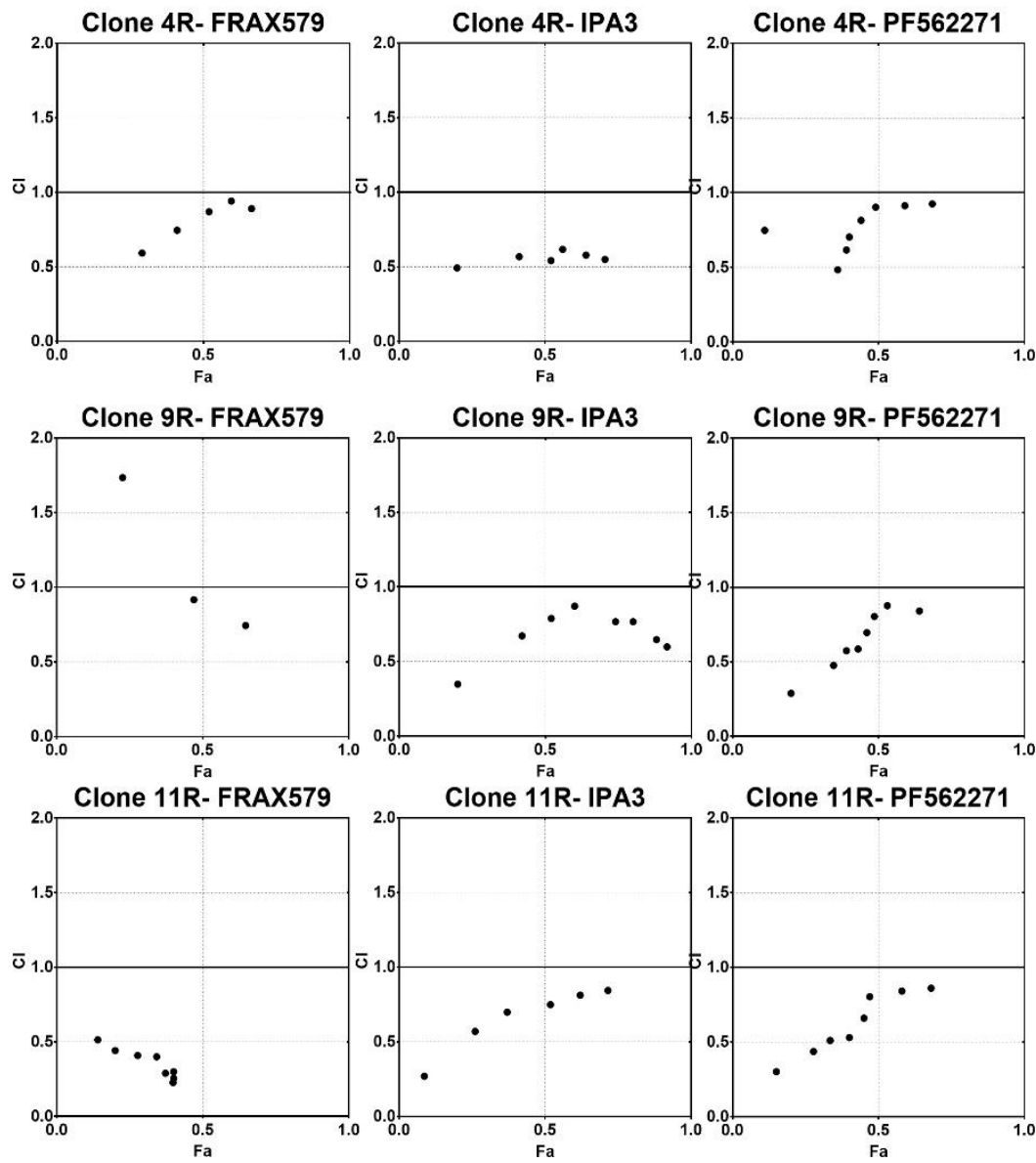

Figure 62: Synergistic effect between FGFR1 and PAK1 or FAK co-inhibition in FGFR1 inhibition induced and intrinsic resistant cell lines.

Combination index plots show the $\mathrm{Cl}$ index, which correlates to the nature of interaction between the FGFR1 inhibitor (AZD4547) and PAK1 inhibitor (FRAX579), PAK1 inhibitor (IPA3) and FAK inhibitor PF562271. Fractions of dead cells (Fa) were measured by MTS assay. Combination indexes (Cl) were calculated using CompuSyn software based on Chou-Talalay drug interaction algorithm. $\mathrm{Cl}<1$ for synergistic effect, $\mathrm{Cl}=1$ for additive effect and $\mathrm{Cl}>1$ for antagonistic effect. 
In order to test the effect of FGFR1 and FAK co-inhibition on Akt activation, I blotted protein lysates from singly or doubly treated cells on nitrocellulose membranes and analyzed the fold change of Akt and PRAS40 phosphorylation compared to DMSO treated cells. Western blot showed reduction in Akt and PRAS40 (substrate of Akt) phosphorylation upon co-inhibition of FGFR1 and FAK compared to control cells (Figure 63).

\section{Clone 4R}

Clone 9R

Clone 11R

H520

DMSO FGFR1i FAKi Combo DMSO FGFR1i FAKi Combo DMSO FGFR1i FAKi Combo DMSO FGFR1i FAKi Combo

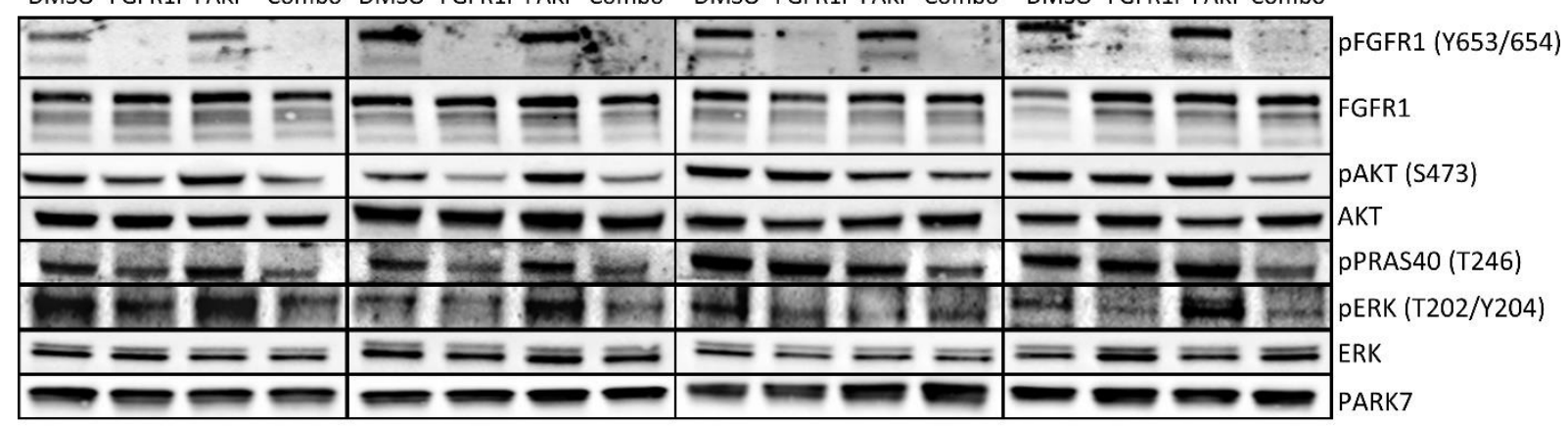

pAkt (Ser473)/PARK7

PPRAS40 (T246)/PARK7
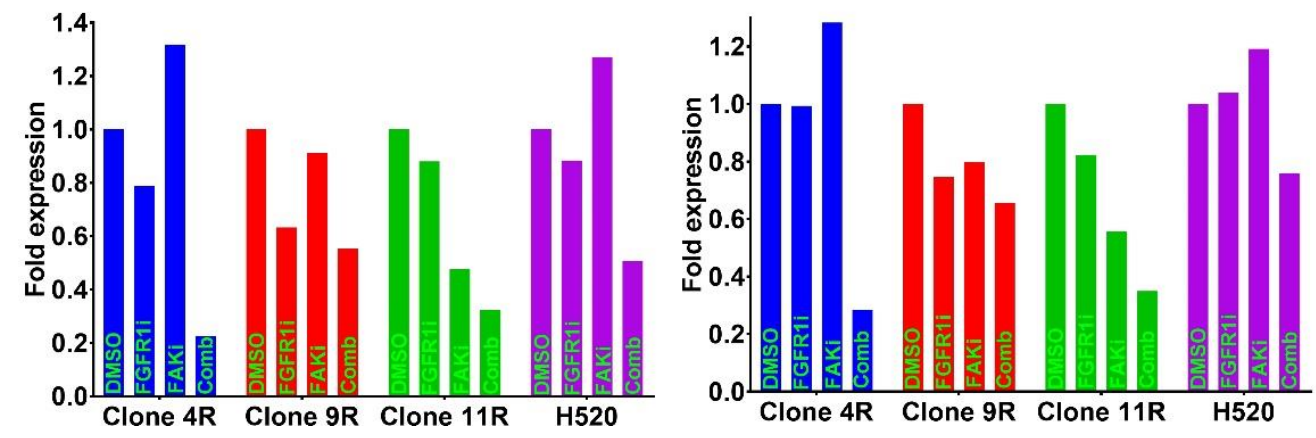

Figure 63: Immunoblotting for FGFR1 and FAK co-inhibition in resistant cells.

Western blot shows levels of phosphorylated FGFR1, Akt and PRAS40 proteins under single inhibition

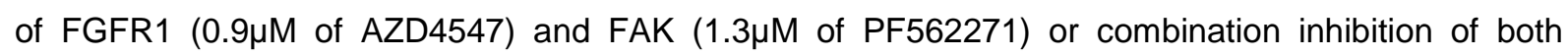
inhibitors. Charts show fold change of Akt and PRAS40 phosphorylation levels compared to the DMSO treated cells after normalizing to the housekeeping protein PARK7.

Finally, to test the effect of combining CD44 knockdown with FGFR1 inhibition in resistant cells, I transfected the intrinsic and induced resistant cells with a functionally verified siRNA targeting CD44 or a scrambled siRNA. Western blot confirmed the efficiency of CD44 knockdown at 100nM. Afterwards, resistant cells were incubated with either DMSO and siControl, FGFR1 and siControl, DMSO and siCD44 or FGFR1 and siCD44 for nine days. MTS proliferation assay showed significant reduction in proliferation of resistant cells under co-treatment with the FGFR1 inhibitor (AZD4547) and CD44 siRNA (Figure 64). 
Clone 9R
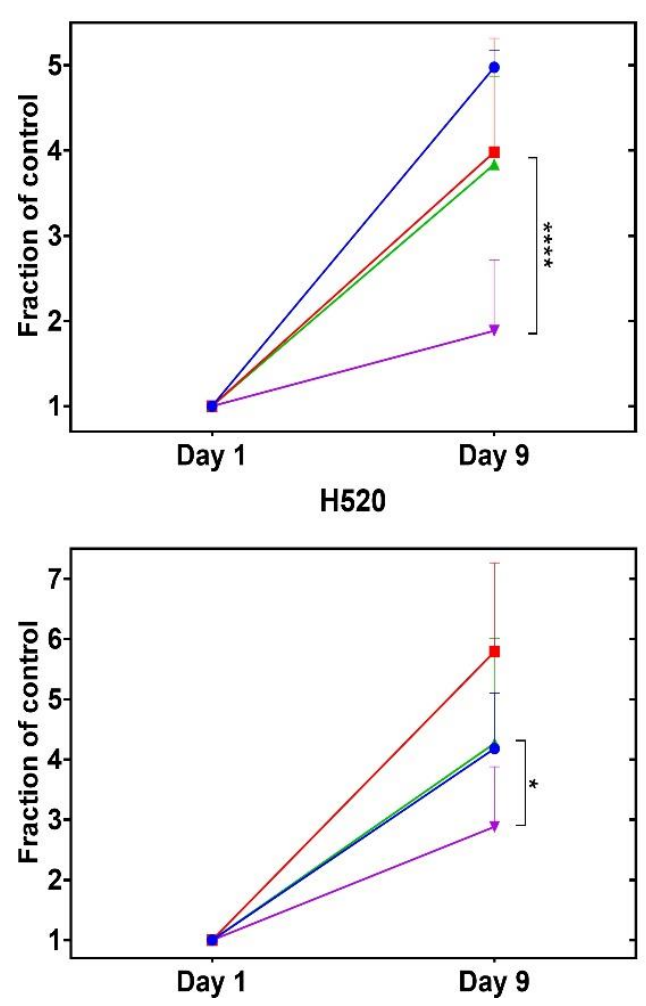

Clone 11R
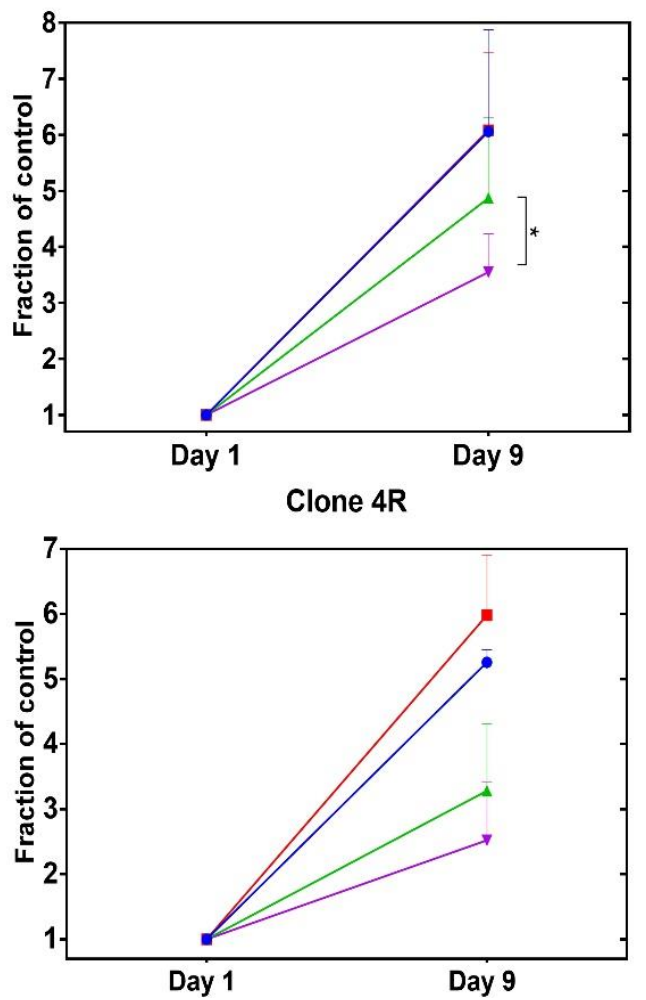

$\mathrm{DMSO}+$ siControl

DMSO + siCD44

FGFR1i+ siControl

F FFR1i + siCD44

Figure 64: Combination between FGFR1 inhibition and CD44 knockdown in FGFR1 inhibition resistant lung cancer cells.

Combination between FGFR1 inhibition through AZD4547 and CD44 knockdown through a siRNA transfection. Experiments were conducted in triplicates. $P$ values were denoted as ${ }^{*}=p \leq 0.05$ and $* * \star *$ $=p \leq 0.0001$. 


\section{Discussion}

\subsection{Molecular targets in lung cancer}

Lung cancer remains the primary cause of cancer related death worldwide (IARC, 2018). Lack of early symptoms is a characteristic of lung cancer, which leads to diagnosis at late and metastatic stages. Widespread screening of high-risk groups has shown that only $15 \%$ of newly diagnosed patients had localized lung cancer tumors, while about $57 \%$ of the newly diagnosed patients suffered metastatic tumors (V. W. Chen et al., 2014). The five-year survival rate of metastatic lung cancer is only about 2-5\% (Bozinovski et al., 2016; NIH, 2018). Aggressiveness and spread of lung cancer have limited the possibility of surgical intervention on the one hand, and required more effective therapy than conventional systemic chemotherapy on the other hand.

Molecular therapy has arisen as the new promising cure for cancer in general and for lung cancer specifically. Molecular therapy depends on targeting molecular alterations (e.g., gene mutation, amplification and rearrangement) with specific drugs. The specificity of the drug to the molecular target alongside with the specificity of the molecular target to the tumor cells made this approach quite effective with minimal side effects (Kurzrock et al., 2020).

Multiple specific targets have been discovered in lung cancer. The US Food and Drug Administration (FDA) has approved therapies against epidermal growth factor receptor (EGFR) mutations like Erlotinib, Afatinib and Gefitinib. Molecular therapies against ALK gene rearrangements like Crizotinib, Ceriitinib and Alectinib have been approved by the FDA as well (Travis et al., 2013). While the aforementioned molecular targets and therapies have proven effectiveness and improved patients' prognosis, they all belong to only one class of lung cancer, which is adenocarcinoma (AC) of non-small cell lung cancer (NSCLC). Adenocarcinoma of the lung forms about $40 \%$ of all lung cancer histology. On the other side, squamous cell lung cancer (SQCLC, around $30 \%$ of lung cancer) and small cell lung cancer (SCLC, around 15\% of lung cancer) completely lack approved molecular targets or molecular therapies (Arbour \& Riely, 2019; Bozinovski et al., 2016; Zappa \& Mousa, 2016). Collectively, previous reasons led to the urge to find new molecular targets in SQCLC and SCLC lung cancer groups. 
Fibroblast growth factor receptor 1 (FGFR1) gene amplification is an emerging promising molecular target in SQCLC and SCLC. New specific inhibitors (e.g., AZD4547 and BGJ398) have been developed to target FGFR1 amplification in designated lung cancer patients who harbor an FGFR1 gene amplification (Katoh, 2019). Phase I and phase II clinical trials of the two inhibitors (AZD4547 and BGJ398) have proven safety and efficacy concept of these inhibitors in the designated patients. However, modest partial response results $(7-11 \%)$ have raised questions about the appropriate patients' selection criteria for these molecular targets and the resistance mechanisms that do exist in FGFR1 amplified patients or develop due to long drug exposure (Aggarwal et al., 2019; Nogova et al., 2017; Paik et al., 2017).

In this thesis, I try to improve the selection criteria for lung cancer patients who could benefit from FGFR1 specific inhibition. Moreover, I try to understand the molecular mechanisms that underline intrinsic and induced resistance to FGFR1 inhibition in SQCLC and SCLC cells with the aim of discovering new molecular targets that sensitize resistant cells.

\subsection{Studying prevalence of FGFR1 gene amplification in SQCLC and SCLC primary patient samples}

In order to use FGFR1 gene amplification as a molecular target in lung cancer, the amplification prevalence had to be widely screened and established among the patients. For this aim, a cohort of 421 lung cancer patient samples was collected. The cohort consisted of 208 patients diagnosed with SQCLC, 121 patients diagnosed with AC and 45 patients diagnosed with SCLC. FGFR1 gene amplification was tested using fluorescence in situ hybridization (FISH) technique. FISH analysis showed $23 \%$ and $8 \%$ prevalence of FGFR1 gene amplification in SQCLC and SCLC groups, respectively. The amplification prevalence in SQCLC and SCLC groups was comparable to previously publicized reports (15-22\% in SQCLC and 5-8.7 in SCLC) (Monaco et al., 2016; Schultheis et al., 2014; Seo et al., 2014; Weiss et al., 2010). In AC group, FGFR1 gene amplification was not screened due to reported scarcity of FGFR1 amplification in this group (0-3\%) (Cihoric et al., 2014; Elakad et al., 2020; Russell et al., 2014; Schildhaus et al., 2012). 
Next, I correlated FGFR1 gene amplification levels in SQCLC and SCLC patients to patient's overall survival. Kaplan-Meier curves showed that no significant correlation existed between the two factors ( $p$-values $=0.726$ and 0.689 in SQCLC and SCLC groups, respectively). Hence, FGFR1 gene amplification status cannot predict patients' prognosis on its own. These results confirmed previous studies, which have shown no correlation between FGFR1 amplification and survival in lung cancer patients (Heist et al., 2012; Kohler et al., 2012; Sasaki et al., 2012) over studies, which have shown significant correlation (Cihoric et al., 2014; Seo et al., 2014; Yang et al., 2013).

\subsection{Validating immunohistochemistry technique and antibody}

In the interest of screening FGFR1 gene expression among the lung cancer patient cohort, tissue microarrays of patient tissue samples were stained against FGFR1 antibody using immunohistochemistry $(\mathrm{IHC})$ technique. Immunohistochemistry is a robust diagnostic and prognostic technique in clinical pathology. Treatment strategies in different tumors can depend mainly on the results of IHC staining (e.g., ER-alpha and HER2 in breast cancer) (Bordeaux et al., 2010). However, the accuracy of the technique relies primarily on the specificity of the antibody used and the staining protocol. According to the FDA, validation of the antibody requires proving sensitivity, selectivity and reproducibility (Bordeaux et al., 2010). Therefore, the validation of the FGFR1 antibody and the staining technique was an essential step prior to screening FGFR1 expression prevalence. To validate the sensitivity of the FGFR1 antibody, I stained human tissues samples of tonsil and gallbladder and compared their results to literature. Tonsil tissue showed weak FGFR1 signal, while gallbladder tissue showed strong FGFR1 signal. These results correlated to published results in FANTOM5 and HPA databases (Lizio et al., 2015; Uhlen et al., 2015).

To validate the selectivity of the antibody, I used CRISPR-Cas9 technique to knock out FGFR1 gene in H1703 SQCLC cell line. Two different guide RNAs (gRNAs) were designed to target and completely delete exon number 14 in FGFR1 gene causing a frameshift mutation and disruption of the protein. Success of the knockout was validated using Sanger sequencing and western blotting. Immunocytochemistry (ICC) staining of the control and the knockout $\mathrm{H} 1703$ cell line showed a complete negative stain in the knockout, which proved the selectivity of the antibody. 


\subsection{Studying prevalence of FGFR1 expression in AC, SQCLC and SCLC primary patient samples}

The validated FGFR1 antibody was used to stain 171, 114 and 44 SQCLC, AC and SCLC patient tissue samples, respectively. IHC signals showed strong FGFR1 expression in 9\%, 35\% and 4\% in SQCLC, AC and SCLC samples, respectively. In the SQCLC group, FGFR1 protein expression was comparable to previously published results (i.e., 10\% prevalence) (Kohler et al., 2012). In AC group, 35\% FGFR1 expression prevalence was slight higher than previous reports (31\%), while in SCLC group, previous reports have reported between 7.2 and $43.7 \%$ FGFR1 expression (Kohler et al., 2012; Yang et al., 2013; L. P. Zhang et al., 2015). The variation of reported prevalence of FGFR1 expression could originate from differences in the antibody used, cutoff values for positive and negative results and patients characteristics (Elakad et al., 2020).

Afterwards, I checked if FGFR1 expression level could have a prognostic value in the three tested lung cancer groups. Kaplan-Meier survival curves showed no significant correlation between FGFR1 expression levels and any of the tested groups with $p$ values of $0.922,0.724$ and 0.443 in SQCLC, SCLC and AC groups, respectively. The results validated the non-significant correlation between FGFR1 protein expression and lung patients overall survival published before (Behrens et al., 2008; Heist et al., 2012).

\subsection{Correlation between FGFR1 gene amplification and protein expression in lung cancer primary patient samples and cell lines}

In order to study FGFR1 gene amplification as a predictive biomarker for molecular target therapy in lung cancer, the correlation between FGFR1 gene amplification and protein expression has to be well understood. Investigating such correlation can help us indicate more accurate selection criteria for FGFR1 inhibition in clinical trials. FGFR1 gene amplification and protein expression were correlated in 129 SQCLC and 36 SCLC patient tissue samples as well as in 14 lung cancer cell lines. The correlation in patient samples was based on comparing $\mathrm{FISH}$ to $\mathrm{IHC}$ analyses. In the lung cancer cell lines, the correlation was based on comparing FISH to ICC and 
western blotting analyses. Chi-square test showed non-significant correlation between the two parameters with p-values of 0.54, 0.99 and 0.09 in SQCLC, SCLC and lung cancer cell lines, respectively. Again, these results do promote previous reports, which have shown no correlation between the two parameters over reports, which have shown significant correlation (Pros et al., 2013; von Massenhausen et al., 2013; Y. Wang et al., 2015).

While clinical trials of FGFR1 inhibitors have relied mainly on FGFR1 amplification and not expression as patients' selection criteria, the inconsistency between the two parameters could account for the modest results that were observed in these trials.

\subsection{Building a lung cancer cell lines model to study mechanisms of FGFR1 inhibition resistance}

In order to study the native or intrinsic resistance mechanisms against FGFR1 inhibition, an appropriate cell lines model had to be established. The first characteristic of the model is that it contains sensitive and resistant cell lines to FGFR1 inhibition. To test sensitivity to FGFR1 inhibition, 21 lung cancer cell lines were incubated with increasing concentrations of the FGFR1 inhibitor AZD4547 for 96 hours. Viability of the cells was measured through MTS assay. Cell lines represented the three histological types: squamous cell lung cancer, small cell lung cancer and large cell lung cancer. These types of lung cancer were selected as they constitute the main histological types of lung cancer that are being targeted by FGFR1 inhibitors (Paik et al., 2017).

The study model included cell lines harboring FGFR1 gene amplification and strong protein expression, which formed the testing group. In the control group, two lung cancer cell lines were used, which did not harbor FGFR1 gene amplification or strong protein expression. To test FGFR1 gene amplification in the cell lines, they were embedded into cell blocks and then stained against the FGFR1 gene and centromere 8 as a control and tested through FISH technique. FISH technique was chosen to test for FGFR1 gene amplification as this technique is considered the regular gene amplification diagnostic technique for patients' tissue samples in routine diagnostics. FISH analysis showed that the cell lines: H520, H1703, DMS114 and H1581 were positive for FGFR1 gene amplification, while the cell lines: HCC15, H2170 and LK2 
were negative. FISH analysis results were supported by previous analyses, which have shown the same amplification scheme among the seven cell lines using realtime PCR and single nucleotide polymorphism (SNP) array based gene copy number assays (Dutt et al., 2011; Guffanti et al., 2017).

FGFR1 protein expression levels were assayed through western blotting and immunocytochemistry using two different anti-FGFR1 antibodies. FGFR1 expression level analyses showed that H1703, H520, H1581, DMS114 and LK2 cell lines demonstrated strong expression of FGFR1 protein, while HCC15 and H2170 cell lines demonstrated very weak FGFR1 expression. These findings were supported by previous studies, which assayed mRNA expression levels using real-time PCR and protein levels using western blotting (Dutt et al., 2011; Rooney et al., 2016).

Correlating FGFR1 gene amplification level and protein expression level, I found that LK2 cell line showed normal gene copy number and strong FGFR1 protein expression at the same time. FGFR1 protein expression of LK2 cell line was comparable to other cell lines with high FGFR1 gene copy number amplification. The inconsistent correlation between FGFR1 gene amplification and protein expression supports the previously discussed correlation in primary patient samples. Interestingly, MTS viability assay showed that LK2 cell line is a highly sensitive cell line to FGFR1 inhibition with an IC50 $<100 \mathrm{nM}$. Sensitivity of LK2 cell line to FGFR1 inhibition suggests that FGFR1 protein expression could shape a more accurate selection criteria for FGFR1 inhibition than gene amplification. Hence, lung cancer patients without FGFR1 amplification could benefit from FGFR1 inhibition therapy if they harbor FGFR1 strong protein expression.

\subsection{Validation of AZD4547 tyrosine kinase inhibition activity and specificity to FGFR1}

Despite high specificity reported of AZD4547 as an FGFR1 inhibitor, it was essential to expand validation of the model's response pattern beyond a single inhibitor. BGJ398 is a selective pan-FGFR1 inhibitor developed by Novartis (Katoh, 2019). To validate the sensitivity scheme of the cell lines in my model to FGFR1 inhibition, I treated the cell lines with the FGFR1 specific inhibitor BGJ398 for 96 hours and measured the viability through MTS viability assay. Sensitivities of all cell lines under 
AZD4547 inhibition were comparable to those under BGJ398 inhibition, which validated the sensitivity scheme of the cell lines in the model. AZD4547 and BGJ398 inhibitors showed that $\mathrm{H} 1581$, DMS114 and LK2 cell lines are highly sensitive to FGFR1 inhibition with IC50 < $100 \mathrm{nM}$. On the other side, H1703, H520, HCCC15 and $\mathrm{H} 2170$ cell lines are insensitive to the inhibition with IC50 $>5 \mu \mathrm{M}$. These results confirmed findings of other reports, which tested the sensitivity of the mentioned cell lines to FGFR1 inhibition (S. M. Kim et al., 2016; Kotani et al., 2016; Malchers et al., 2017). Sensitivities of cell lines to FGFR1 inhibition were measured using two different viability assays (MTS assay and Muse viable cell count assay).

Receptor tyrosine kinase inhibitors are prone to a degree of unspecific effects due to off-target inhibition. To ensure that the response pattern of my model reflected their dependency on FGFR1 signaling rather than other targets, I knocked down FGFR1 via small interfering RNA (siRNA). FGFR1 knockdown was evaluated through western blotting using anti-FGFR1 antibody. Cell proliferation assay showed significant reduction in proliferation of DMS114, H1581 and LK2 cell lines during fifth and sixth days compared to scrambled control siRNA group. On the other side, proliferation of control-siRNA and FGFR1-siRNA groups in HCC15, H2170, H1703 and H520 cell lines were comparable. FGFR1 knockdown results suggested that the sensitivity scheme of the tested cell lines originated from their dependence on FGFR1 signaling rather than on unspecific effects of the inhibitors used.

\subsection{Activated signaling pathway in sensitive and resistant cell lines to FGFR1 inhibition}

Under normal healthy conditions, FGFR1 regulates vital processes in the body like cell proliferation, differentiation and survival. FGFR1 controls these functions through regulating the activation of three main pathways: MAPK-ERK, PI3K-Akt and JAKSTAT pathways (Malchers et al., 2017). Dysregulation of these main pathways is responsible not only for tumorigenesis induced by FGFR1, but also for resistance to FGFR1 inhibition (Kotani et al., 2016). In order to understand regulation of the main pathways in FGFR1 signaling under sensitive and resistant conditions, cellular lysates of DMSO or AZD4547 treated cell lines were blotted on membranes and stained against the main effectors in FGFR1 signaling. Treatment with $0.5 \mu \mathrm{M}$ AZD4547 for 3 hours showed complete decline in phosphorylated FGFR1 signal at 
tyrosine site number 654 (Y654), which validated the function of the inhibitor. Efficacy of FGFR1 inhibition through AZD4547 was further validated through the reduction of activated MEK and ERK signals in sensitive and resistant cell lines while total unphosphorylated MEK and ERK signals remained unchanged. The main difference between FGFR1 signaling in sensitive and resistant cell lines was seen in the Akt pathway. The two resistant cell lines (H520 and H1703) showed highly activated signals of Akt at sites of Serine 473 (Ser473) and Threonine 308 (Thr308), while total Akt remained comparable among all cell lines.

To extend the analysis of FGFR1 signaling in sensitive and resistant cell lines, levels of RNA expression were analyzed through Archer FusionPlex CTL Panel ${ }^{\circledR}$. Archer CTL panel is a site directed RNA sequencing technique, which tests the expression levels of 36 genes associated with lung and thyroid cancers (Benayed et al., 2019; Chang et al., 2019). RNA sequencing showed that the main difference between the three groups of cell lines (control, sensitive and resistant groups) was the elevated expression levels of FGFR1 in resistant and sensitive cells compared to control cells. The second observation out of the RNA sequencing analysis was the significant elevation of Akt1 signal in $\mathrm{H} 520$ resistant cell line and the slight elevation of the same signal in H1703 resistant cell line compared to the three cell lines in the sensitive group. While western blot analysis showed uniformity between total Akt signals in all cell lines, RNA sequencing showed elevated RNA expression of Akt1 in resistant cell lines. This apparent conflict could be explained by the specificity of the RNA sequencing to Akt1 expression, while the antibody used in western blotting was a pan-Akt antibody, which binds to all Akt isoforms (Akt1, Akt2 and Akt3). The last observation of the RNA sequencing analysis was the absence of any other significant distinction between the sensitive and resistant groups in any of the 36 genes associated with lung and thyroid cancers.

Collectively, signaling pathway analyses between sensitive and resistant cell lines suggested that Akt activation could play an important role in the resistance to FGFR1 inhibition. Akt pathway is a main survival pathway, which regulate basic functions in the cells like cell proliferation, apoptosis, gene transcription and cell migration. These processes construct the main players in developing any resistance against general or directed therapies (Huang \& Hung, 2009). Consequently, in tumor treatment, activation of Akt signaling has been reported to play part in chemotherapy resistance, 
DNA-targeted therapy resistance and directed small molecule inhibitors resistance (Avan et al., 2016; Cassinelli et al., 2013; Huang \& Hung, 2009; Jacobsen et al., 2017).

\subsection{Activation of Akt in FGFR1 amplified and FGFR1-expressing primary SQCLC tissue samples}

To check if Akt overactivation is a real event that takes place in primary patient samples and not an exclusive behavior of cell lines, I stained 32 FGFR1-amplified and 45 FGFR1- expressing SQCLC primary tissue sample for pAkt (Ser473). Immunohistochemistry analysis (through $\mathrm{H}$-score system) showed wide range of variation in Akt phosphorylation among patient samples. Some patients completely lacked any phosphorylation of Akt (H-score = zero), while other patients showed strong phosphorylation of Akt $(\mathrm{H}$-score $=300)$. The majority of patients ranged between the two margins. This finding does add weight to the proposed role of Akt activation in FGFR1 inhibition resistance. Nevertheless, these patients were not treated with FGFR1 inhibitors, hence a direct connection between Akt activation level and patients' response to FGFR1 inhibition could not be concluded from this data.

\subsection{Combining FGFR1 and Akt inhibition to sensitize resistant cells to FGFR1 inhibition}

In cancer treatment, combination therapy is a major module where patients are treated with more than one therapy simultaneously. Combined therapies are usually accompanied by better prognosis and outcome for two main reasons. Firstly, the synergistic or additive effect of the two therapies is stronger than the monotherapy. Secondly, combined therapies can compensate for patient-to-patient variability (Palmer \& Sorger, 2017). In specific targeted drugs, combined therapies usually target the same pathway or a resistance pathway of the leading therapy. Combination therapy usually utilizes one or more FDA approved therapies together, hence increasing efficiency of treatment and reducing time needed for approvals at the same time (Bayat Mokhtari et al., 2017). Accordingly, combining Akt inhibition to FGFR1 inhibition could have a synergistic effect, inhibit the resistance pathway and improve the outcome. 
In order to test the effect of combining Akt and FGFR1 inhibition, I treated the FGFR1 inhibition resistant cell lines (H520 and H1703) with both AZD4547 (FGFR1 inhibitor) and AZD5363 (Akt inhibitor) simultaneously. AZD5363 is an ATP-competitive Akt inhibitor, which is currently in phase II clinical trials (Banerji et al., 2018; Schmid et al., 2018). MTS viability assay showed a strong and significant reduction of resistant cells' viability under the combined therapy. To test the interaction between the two inhibitors, I used the Chou-Talalay combination index $(\mathrm{Cl})$. Chou-Talalay method for calculating multiple drugs interactions is a widely recognized and validated method, which is based on median-effect equation and can differentiate between synergistic, additive and antagonistic effects represented by $\mathrm{Cl}<1, \mathrm{Cl}=1$ and $\mathrm{Cl}>1$, respectively (Chou, 2006, 2010). Cl indexes showed strong synergistic effects between FGFR1 and Akt inhibitors in the resistance cell lines.

These results proposed a hypothesis, which states that Akt activation is an intrinsic resistance mechanism in FGFR1-amplified SQCLC lung cancer cells to FGFR1 inhibition. In other words, high level of Akt activation does pre-exist in some lung cancer cells before receiving any FGFR1 inhibition. Cells with high Akt activation are resistant to FGFR1 inhibition and could be targeted using Akt inhibitors combined with FGFR1 inhibitors.

\subsection{Inducing Akt activation in sensitive lung cancer cell lines to FGFR1 inhibition}

To validate the tendency of Akt activation to induce FGFR1 inhibition resistance, I transfected the three sensitive cell lines (H1581, DMS114 and LK2) with constitutively active Akt plasmids. The plasmids do express myristoylated Akt (MyrAkt), which is based on attachment of a myristoyl group (lipid group) to the Akt leading to its localization to the cell membrane and hence activation (Kohn et al., 1996). Overactivation of Akt was validated by western blotting, which showed high elevation of pAkt at Ser473 in H1581 cell line, medium elevation in pAkt signal in LK2 cell line, while DMS114 cell line showed only minimal activation of Akt. MTS viability assay revealed that overactivation of Akt significantly induced FGFR1 inhibition resistance compared to controls. Resistance of mutant-Akt cells was the highest in H1581 followed by LK2, while DMS114 did not show difference in the response to FGFR1 inhibition. The variation in induced resistance between the three cell lines 
could be attributed to the magnitude of mutant-Akt activation in these cells shown by western blotting. Again, combination inhibition between Akt and FGFR1 inhibitors succeeded to synergistically reverse the resistance induced by activated Akt and reduce cells' viability in H1581 and DMS114 cell lines after treatment.

\subsection{Induction of FGFR1 resistance in FGFR1 inhibition sensitive batch cell lines}

Tumor cells are in a constant movement to induce their proliferation, reduce their cell death and compensate for essential pathways inhibited by any therapeutic elements. This movement leads cancer cells to acquire resistance against targeted drugs after long exposure. Resistance induced in cancer cells due to long exposure to treatment is defined as induced or acquired resistance (Holohan et al., 2013). Another hypothesis does exist, which explains the mechanism of acquired resistance slight differently. The hypothesis describes tumors as huge group of heterogeneous cells, which could contain very few cells that are originally resistant to the therapeutic agent. These few or single resistant cells get selected under long incubation period of the therapeutic agent (Holohan et al., 2013; Xuan Wang et al., 2019).

In order to study induced resistance to FGFR1 inhibition in my model, I exposed the three sensitive cell lines (H1581, DMS114 and LK2) to the FGFR1 inhibitor (AZD4547) for four months. MTS viability assay validated the high level of resistance induced in the originally sensitive cell lines to FGFR1 inhibition. To test the activated signaling pathways in the induced resistance cell lines, protein lysates of the cell lines were blotted on membranes and analyzed through western blotting technique. The immunoblotting was repeated at least three times for each of the cell lines with different biological replicates. Results showed large diversity in the ERK-MAPK and PI3K-Akt pathways between each of the replicates. I attributed the inconsistency of the activated signaling pathways between the replicates to the cell lines batch-effect. Batch-effect meant that each of the cell lines enclosed heterogeneous group of cells with different mechanisms of resistance developed and fluctuated inside. The versatile mechanisms of acquired resistance developed in a single lung cancer cell line have been shown before in adenocarcinoma targeted with EGFR inhibitors (Jacobsen et al., 2017). 


\subsection{Inducing FGFR1 resistance in single clones of FGFR1 inhibition sensitive cell lines}

In order to surpass the batch-effect of acquired resistance cell lines, I sorted H1581, DMS14 and LK2 cell lines into single clones and then treated them with rounds of high concentration of FGFR1 or fresh growth medium. Due to high vulnerability of single cells, the process of resistance induction lasted for ten months. A total of 14 , 17 and 17 resistant single clones were successfully obtained from H1581, DMS114 and LK2 cell lines, respectively. Western blot analysis did confirm the variation in activated signaling pathways in resistant single clones, which shared a parental cell line. Akt activation was found in 21.4\%, 29.4\% and 5.9\% in H15851, DMS114 and LK2 single clones compared to parental controls, respectively. On the other side, ERK activation was found in 14.3\%, 5.9\% and 25.5\% in H15851, DMS114 and LK2 single clones compared to parental controls, respectively.

Combining Akt inhibition and FGFR1 inhibition, successfully and significantly sensitized the three $\mathrm{H} 1581$ resistant single clones with Akt activation (clones 4, 9 and 11) to FGFR1 inhibition using two different viability assays. Chou-Talalay analysis showed the interaction between FGFR1 and Akt inhibitors to be a strong synergistic interaction $(\mathrm{Cl}=0.1-0.03)$.

To analyze signaling pathways in the three resistant single clones ( $\mathrm{C} 4, \mathrm{C} 9$ and $\mathrm{C} 11)$, expressed RNA levels were measured using Archer CTL directed panel and protein levels were assayed using western blotting. On RNA expression level, RNA sequencing showed upregulation in FGFR1 expression and downregulation of GNAS to be the only two significant differences between resistant clones and their parental control cell line.

On protein level, western blotting confirmed the activation of Akt in the three clones (at least at Ser473 site). Literature show that a decline in PTEN levels (an Akt suppressor) is usually accompanied by elevation of Akt activation, which was the case in C9 and C11 resistant clones (Georgescu, 2010). Interestingly, PTEN levels in C4 clone were comparable to the parental H1581 cell line. Western blotting showed that treatment with $0.5 \mu \mathrm{M}$ AZD4547 was sufficient to inhibit phosphorylation of FGFR1 (Y653/Y654) in the parental cell line as well as the three single clones equally. The last observation suggested that overexpression of FGFR1 was not the 
reason behind FGFR1 inhibition resistance in the three single clones, which added more weight to the role of Akt overactivation in inducing the resistance.

PRAS40 (Akt1S1), an important substrate of Akt and a subunit of mTOR complex 1 (mTORC1), is a downstream effector of Akt with strong tumor proliferative and survival capabilities (Lv et al., 2017; Qi et al., 2020). PRAS40 was strongly activated in all of the three resistant clones. On the other side, MEK signals were activated only in C4 clone and largely inhibited in C9 and C11 clones compared to parental control. Likewise, activated ERK signals were lost in $\mathrm{C} 9$ and $\mathrm{C} 11$ clones but not in $\mathrm{C} 4$ clone compared to parental control. Taken together, western blot analysis suggested that $\mathrm{C} 4, \mathrm{C} 9$ and $\mathrm{C} 11$ clones gained their resistance to FGFR1 inhibition through activating the Akt-PRAS40 survival pathway.

\subsection{Sample preparation for LC-MS/MS phosphoproteomic analysis in FGFR1 inhibition resistant lung cancer cell lines}

Liquid chromatography coupled to mass spectrometry (LC-MS/MS) is a robust quantitative method that can accurately measure levels of proteins in tested samples. Applying LC-MS/MS to tumor research can help allocating signaling pathways responsible for tumor initiation, progression, metastasis as well as resistance to tumor therapies (Bohnenberger et al., 2018; Corso et al., 2016). Analyzing protein levels in tumor cells reveals more information on the functional level than analyzing DNA and RNA nucleic acid levels. Enriching the proteomic analysis for phosphorylated proteins can help us to precisely understand functional activated and deactivated specific signaling pathways in tumor cells (H. Wang et al., 2016).

In the current project, I used LC-MS/MS phosphoproteomic analysis in order to identify resistance mechanisms to FGFR1 inhibition in lung cancer cells with the aim of finding new molecular targets that could sensitize resistant cells. The phosphoproteomic analysis was carried out with a special focus on validating and stratifying Akt pathway activation as a resistance pathway. I designed the model to include one control cell line and three groups of resistant cell lines. Firstly, H1581 parental cell line was analyzed as the parental sensitive control cell line. Secondly, H520 cell line was used as an intrinsic or native resistant SQCLC cell line. Thirdly, mutant-Akt H1581 cell line was used as a genetically induced resistant cell line. 
Finally, $\mathrm{H} 1581$ resistant clones $\mathrm{C} 9$ and $\mathrm{C} 11$ were used as induced resistant cell lines. Clones number 9 and 11 were specifically selected from $\mathrm{H} 1581$-induced resistance clones as they showed elevation in Akt activation compared to the parental cell line.

The aforementioned model structure, with the help of the mutant-Akt cell line, was able to not only differentiate between resistance mechanisms in native and induced cell lines, but also between activated signaling pathways upstream and downstream of Akt.

For the aim of exploring the direct effect of AZD4547 inhibition, all cell lines were analyzed under control conditions (DMSO-treated) and under FGFR1 inhibition conditions (AZD4547-treated). DMSO-treated cells were cultured in medium heavy SILAC medium, while AZD4547-treated cells were cultured under heavy heavy SILAC medium. To control the quantification of DMSO and AZD4547 treated cells, we created a super-SILAC control. Super-SILAC control is composed of a mixture of different cell lines from the same tumor type mixed in equal ratios. The mixture of cell lines can more accurately represent the proteome of the tumor than a single cell line and hence improves the overall quantification accuracy (Geiger et al., 2010). To create a super-SILAC control, cell lysates out of four lung cancer cell lines were mixed in equal ratios and cultured in normal growth medium (light medium). Moreover, cell lysates were divided into two groups: global proteome group (GPome) for the global phosphorylated peptides and tyrosine-enriched group (pYome) for mainly tyrosine phosphorylated peptides.

\subsection{Quality control of LC-MS/MS phosphoproteomic analysis results}

To analyze the MS data, I used the Perseus software (version: 1.6.10.43), which is an open source mass spectrometry analysis tool (Tyanova \& Cox, 2018; Tyanova et al., 2016). Numeric Venn diagrams showed that numbers of quantified phosphopeptides, in GPome group, were 6044, 6476, 5650, 6088 and 6032 in H1581, mutant-Akt, H520, C9 and C11 cell lines, respectively. In pYome group, quantified phosphopeptides were 312, 364, 310, 290 and 192 in H1581, mutant-Akt, H520, C9 and C11 cell lines, respectively. In total, 14014 different phosphosites were quantified in the GPome group, while 831 different phosphosites were quantified in 
the pYome group. These high counts of successfully quantified phosphosites on the one side reflected good quality of sample preparation and of data in general and on the other side allowed for more chance of low abundant proteins' quantification (Haupl et al., 2019).

In GPome group, one technical replicate from each biological replicate of C9 cell line showed reduced counts of quantified cites, which was compensated through calculating the average of both technical replicates during the analysis. In pYome group, one technical replicate of only one biological replicate of $\mathrm{C} 11$ cell line showed reduced counts of sites, which was compensated through calculating the average of both technical replicates.

Multi-scatter plots showed that coefficient of determination ( $R$ squared) between biological replicates in the GPome group to range between 0.7 and 0.8 except for H520 cell line in DMSO-treated group (0.4) and C9 cell line in AZD4547-treated group (0.5). In pYome group, $\mathrm{R}$ squared values between biological replicates ranged between 0.7 and 0.9 except for H520 cell line in DMSO-treated group (0.5) and C9 cell line in AZD4547-treated group (0.6). The aforementioned $R$ squared values showed that the quality of the biological replicates was good and the replicates could be used in functional analyses.

Statistical analysis using ANOVA significance test showed 731 and 667 phosphosites to be significantly differentially phosphorylated between the five groups tested in the DMSO-treated and the AZD-treated GPome groups, respectively. In the pYome analysis, ANOVA analyses showed 34 and 86 sites to be significantly different between the five groups tested in DMSO-treated and AZD4547-treated groups, respectively. Histograms in GPome and pYome groups showed the majority of quantified proteins to have narrow distribution, which represented the advantage of using a super-SILAC standard.

\subsection{Activated signaling pathways among different models of FGFR1 inhibition resistant lung cancer cell lines}

Heatmaps of significantly differentiated phosphosites between all biological replicates showed on the one side high quality of the biological replicates and on the other side similarities and differences between the tested groups. Clusters of significant sites 
clearly showed the first degree of similarity to be between the two induced resistant clones $\mathrm{C} 9$ and $\mathrm{C} 11$. The second degree of similarity was between induced ( $\mathrm{C} 9$ and C11) and intrinsic (H520) resistant cells compared to H1581 control and mutant-Akt cell line. The large degree of similarity in phosphoproteomic profiles of H1581-C9, H1581-C11 and H520 cells supports the existence of a common mechanism of resistance to FGFR1 inhibition in lung cancer. Moreover, the latter degree of similarity could be interpreted, to a certain limit, as the difference between upstream and downstream activation of Akt due to its distinction from mutant-Akt cell line.

I examined functional annotations of the significant phosphosites using databases of Sring, DAVID and Gene cards (Dennis et al., 2003; Fishilevich et al., 2016; Szklarczyk et al., 2019). Moreover, I explored the literature to check for the relevance of these sites in tumor biology. Bioinformatics analysis along with literature review revealed a strong enriched pathway in resistant cell lines compared to the control cell line. Phosphorylation of CD44 at S706 (> 30-fold), FAK at Y576 (> 4-fold), PAK1 at S174 (> 10-fold), Paxillin at S119 (> 2-fold), Afadin at S1182 (>2-fold) and reduction of Fyn phosphorylation at Y222 ( $>2$-fold) phosphosites formed the core of the resistance pathway, upstream Akt, in intrinsic and induced resistant cells compared to parental H1581 and mutant-Akt cell lines.

CD44 is a transmembrane adhesion glycoprotein that regulates essential cellular functions like cell division, migration and cell-cell signaling (Basakran, 2015). Inhibiting CD44 expression has reduced tumor cells proliferation, metastasis and increased chemosensitivity and apoptosis in hepatocellular carcinoma, breast cancer and lung cancer (Basakran, 2015; X. Liu et al., 2019; Xie et al., 2008). In breast cancer, CD44 was shown to induce circulating tumor cells (CTC)-mediated metastasis through activation of PAK 1/2, FAK and Paxillin proteins (X. Liu et al., 2019). On the other side, PAK1 (p21-activated kinase 1) is a member of serine/threonine kinase family, which consists of six members (PAK1-6) (Wu et al., 2016). PAK1 regulates vital processes in the cells like cytoskeleton remodeling, proliferation and survival (Wu et al., 2016). Overexpression and overactivation of PAK1 has been reported in lung, brain, live and kidney tumors (Wu et al., 2016; Ye \& Field, 2012). Activity of PAK1 protein is regulated through Akt, Cdc42 and Rac proteins phosphorylation (Zhou et al., 2003). 
To study resistance-signaling pathways downstream of Akt, I compared the H1581 parental control cell line to all resistant cell lines (including mutant-Akt H1581). Comparison showed activation of different targets responsible for processes of apoptosis inhibition (DAXX, ACIN1, BIM, AVEN and TSC22), promotion of proliferation and survival (SOX2, GAPDH and LMo7) and induction of migration and metastasis (ANXA1 and cortactin). Overactivation of Akt1 at $\mathrm{S} 124$ and its downstream effector PRAS40 at T246 were among overactivated proteins in all resistant cell lines compared to H1581 control cell line. (L. Y. Chen \& Chen, 2003; Colell et al., 2007; de Graauw et al., 2010; Dennis et al., 2003; Eissmann et al., 2013; Fishilevich et al., 2016; Szklarczyk et al., 2019)

\subsection{Validation of PAK1 and FAK as a molecular targets in intrinsic and induced FGFR1 resistant lung cancer cell lines}

Phosphoproteomic analysis presented a core resistance pathway in intrinsic and induced resistant lung cancer cell lines to FGFR1 inhibition. Multiple novel drug targets within the resistance pathway could be tested and validated for their ability of sensitizing lung cancer resistant cells to FGFR1 inhibition. While the only current, direct and selective way to inhibit CD44 is through genetic knockdown, some selective inhibitors are available to target PAK1 and FAK proteins. I used FRAX579 (ATP competitive), IPA-3 (non-ATP competitive) and PF562271 (ATP competitive) selective inhibitors to inhibit PAK1 and FAK in induced resistant cell lines (C4, C9 and $\mathrm{C} 11$ ) as well as in the intrinsic resistant cell lines $\mathrm{H} 520$ and $\mathrm{H} 1703$. MTS viability assay accompanied by Chou-Talalay combination index showed synergistic effect between PAK1, FAK and FGFR1 inhibition in all tested resistant cell lines. Likewise, combination between CD44 knockdown and FGFR1 inhibition showed significant reduction in proliferation of resistant cells compared to single treatments. These results suggested that PAK1, FAK and CD44 are potential novel therapeutic targets in FGFR1 inhibition resistant lung cancer cells.

\subsection{Conclusion}

In the current thesis, FGFR1 gene amplification and protein expression prevalence were screened among 421 SQCLC, SCLC and AC patient samples. FISH analyses 
revealed $23 \%$ and $8 \%$ prevalence of FGFR1 amplification in SQCLC and SCLC groups, respectively. IHC analysis revealed $9 \%, 4 \%$ and $23 \%$ prevalence of FGFR1 protein expression in SQCLC, SCLC and AC lung cancer patients, respectively. Correlating FGFR1 gene amplification and protein expression among the same sets of patients showed non-significant correlation. Hence, the choice of FGFR1 gene amplification as the selective criteria for FGFR1 inhibitors in lung cancer patients should be reconsidered. Signaling pathway analyses between FGFR1-inhibition sensitive lung cancer cell lines on the one side and intrinsic or induced resistant cell lines on the other side showed Akt activation as a significant difference between the two groups. Functional viability assays confirmed synergy effect between FGFR1 inhibitors and Akt inhibitors in FGFR1 inhibition resistant lung cancer cell lines. LCMS/MS phosphoproteomic analysis showed that over phosphorylated CD44, PAK1, FAK, Afadin, Paxillin and Akt formed a common resistance pathway in native and acquired resistant lung cancer cells to FGFR1 inhibition. Chou-Talalay combination index analysis confirmed synergy effect between FGFR1 inhibition combined with PAK1 or FAK inhibition in intrinsic and induced resistant lung cancer cells. CD44 knockdown showed ability to significantly reduce proliferation of resistant cells only when combined with FGFR1 inhibition. Collectively, Akt, PAK1, FAK and CD44 present interesting molecular targets to sensitize resistant lung cancer cells to FGFR1 inhibition.

\subsection{Outlook}

Future experiments should focus on five main lines of study. Firstly, studies should focus on validation of multiple molecular targets found in the resistance pathway like Afadin and Paxillin as sensitizers of lung cancer resistant cells to FGFR1 inhibition. Secondly, interaction between the main effectors in the resistance pathway (e.g., interaction between CD44 and PAK1 and Akt) should be validated in the resistant cells. Thirdly, in vivo models should be used to test and validate the synergistic effect of combining PAK1, FAK and Akt inhibitors to FGFR1 inhibitors in resistant lung cancer cells. Fourthly, phosphorylation levels of CD44, PAK1, FAK and Akt should be screened in SQCLC lung cancer patient samples who were treated with FGFR1 inhibitors and correlated to patients overall survival. Finally, future clinical trials 
should test the combination between Akt, PAK1 and FAK inhibitors and FGFR1 inhibitors in FGFR1 inhibition resistant lung cancer patients. 


\section{References}

Adam, R. M., Mukhopadhyay, N. K., Kim, J., Di Vizio, D., Cinar, B., Boucher, K., . . Freeman, M. R. (2007). Cholesterol sensitivity of endogenous and myristoylated Akt. Cancer Res, 67(13), 6238-6246. doi:10.1158/0008-5472.CAN-07-0288

Aggarwal, C., Redman, M. W., Lara, P. N., Jr., Borghaei, H., Hoffman, P., Bradley, J. D., . . Gandara, D. R. (2019). SWOG S1400D (NCT02965378), a Phase II Study of the Fibroblast Growth Factor Receptor Inhibitor AZD4547 in Previously Treated Patients With Fibroblast Growth Factor Pathway-Activated Stage IV Squamous Cell Lung Cancer (Lung-MAP Substudy). J Thorac Oncol, 14(10), 1847-1852. doi:10.1016/j.jtho.2019.05.041

Ankney, J. A., Muneer, A., \& Chen, X. (2018). Relative and Absolute Quantitation in Mass Spectrometry-Based Proteomics. Annu Rev Anal Chem (Palo Alto Calif), 11(1), 49-77. doi:10.1146/annurev-anchem-061516-045357

Arbour, K. C., \& Riely, G. J. (2019). Systemic Therapy for Locally Advanced and Metastatic Non-Small Cell Lung Cancer: A Review. JAMA, 322(8), 764-774. doi:10.1001/jama.2019.11058

Aslantürk, Ö. S. (2018). In Vitro Cytotoxicity and Cell Viability Assays: Principles, Advantages, and Disadvantages. In Genotoxicity - A Predictable Risk to Our Actual World.

Avan, A., Narayan, R., Giovannetti, E., \& Peters, G. J. (2016). Role of Akt signaling in resistance to DNA-targeted therapy. World J Clin Oncol, 7(5), 352-369. doi:10.5306/wjco.v7.i5.352

Bae, J. H., Lew, E. D., Yuzawa, S., Tome, F., Lax, I., \& Schlessinger, J. (2009). The selectivity of receptor tyrosine kinase signaling is controlled by a secondary SH2 domain binding site. Cell, 138(3), 514-524. doi:10.1016/j.cell.2009.05.028

Banerji, U., Dean, E. J., Perez-Fidalgo, J. A., Batist, G., Bedard, P. L., You, B., . . Schellens, J. H. M. (2018). A Phase I Open-Label Study to Identify a Dosing Regimen of the Pan-AKT Inhibitor AZD5363 for Evaluation in Solid Tumors and in PIK3CA-Mutated Breast and Gynecologic Cancers. Clin Cancer Res, 24(9), 2050-2059. doi:10.1158/1078-0432.CCR-17-2260

Basakran, N. S. (2015). CD44 as a potential diagnostic tumor marker. Saudi Med J, 36(3), 273-279. doi:10.15537/smj.2015.3.9622

Bayat Mokhtari, R., Homayouni, T. S., Baluch, N., Morgatskaya, E., Kumar, S., Das, B., \& Yeger, H. (2017). Combination therapy in combating cancer. Oncotarget, 8(23), 38022-38043. doi:10.18632/oncotarget.16723

Behrens, C., Lin, H. Y., Lee, J. J., Raso, M. G., Hong, W. K., Wistuba, II, \& Lotan, R. (2008). Immunohistochemical expression of basic fibroblast growth factor and fibroblast growth factor receptors 1 and 2 in the pathogenesis of lung cancer. Clin Cancer Res, 14(19), 60146022. doi:10.1158/1078-0432.CCR-08-0167

Benayed, R., Offin, M., Mullaney, K., Sukhadia, P., Rios, K., Desmeules, P., . . Ladanyi, M. (2019). High Yield of RNA Sequencing for Targetable Kinase Fusions in Lung Adenocarcinomas with No Mitogenic Driver Alteration Detected by DNA Sequencing and Low Tumor Mutation Burden. Clin Cancer Res, 25(15), 4712-4722. doi:10.1158/1078-0432.CCR-19-0225

Bethune, G., Bethune, D., Ridgway, N., \& Xu, Z. (2010). Epidermal growth factor receptor (EGFR) in lung cancer: an overview and update. J Thorac Dis, 2(1), 48-51.

Bockorny, B., Rusan, M., Chen, W., Liao, R. G., Li, Y., Piccioni, F., . . Bass, A. J. (2018). RAS-MAPK Reactivation Facilitates Acquired Resistance in FGFR1-Amplified Lung Cancer and Underlies a Rationale for Upfront FGFR-MEK Blockade. Mol Cancer Ther, 17(7), 1526-1539. doi:10.1158/1535-7163.MCT-17-0464

Bohnenberger, H., Kaderali, L., Strobel, P., Yepes, D., Plessmann, U., Dharia, N. V., . . Oellerich, T. (2018). Comparative proteomics reveals a diagnostic signature for pulmonary head-and-neck cancer metastasis. EMBO Mol Med, 10(9). doi:10.15252/emmm.201708428

Bollag, G., Tsai, J., Zhang, J., Zhang, C., Ibrahim, P., Nolop, K., \& Hirth, P. (2012). Vemurafenib: the first drug approved for BRAF-mutant cancer. Nat Rev Drug Discov, 11(11), 873-886. doi:10.1038/nrd3847 
Bordeaux, J., Welsh, A., Agarwal, S., Killiam, E., Baquero, M., Hanna, J., . . Rimm, D. (2010). Antibody validation. Biotechniques, 48(3), 197-209. doi:10.2144/000113382

Bozinovski, S., Vlahos, R., Anthony, D., McQualter, J., Anderson, G., Irving, L., \& Steinfort, D. (2016). COPD and squamous cell lung cancer: aberrant inflammation and immunity is the common link. Br J Pharmacol, 173(4), 635-648. doi:10.1111/bph.13198

Bruske-Hohlfeld, I. (2009). Environmental and occupational risk factors for lung cancer. Methods $\mathrm{Mol}$ Biol, 472, 3-23. doi:10.1007/978-1-60327-492-0_1

Cassinelli, G., Zuco, V., Gatti, L., Lanzi, C., Zaffaroni, N., Colombo, D., \& Perego, P. (2013). Targeting the Akt kinase to modulate survival, invasiveness and drug resistance of cancer cells. Curr Med Chem, 20(15), 1923-1945. doi:10.2174/09298673113209990106

Chae, Y. K., Ranganath, K., Hammerman, P. S., Vaklavas, C., Mohindra, N., Kalyan, A., . . Giles, F. J. (2017). Inhibition of the fibroblast growth factor receptor (FGFR) pathway: the current landscape and barriers to clinical application. Oncotarget, 8(9), 16052-16074. doi:10.18632/oncotarget.14109

Chang, F., Lin, F., Cao, K., Surrey, L. F., Aplenc, R., Bagatell, R., ... Li, M. M. (2019). Development and Clinical Validation of a Large Fusion Gene Panel for Pediatric Cancers. J Mol Diagn, 21(5), 873883. doi:10.1016/j.jmoldx.2019.05.006

Chen, L. Y., \& Chen, J. D. (2003). Daxx silencing sensitizes cells to multiple apoptotic pathways. Mol Cell Biol, 23(20), 7108-7121. doi:10.1128/mcb.23.20.7108-7121.2003

Chen, V. W., Ruiz, B. A., Hsieh, M. C., Wu, X. C., Ries, L. A., \& Lewis, D. R. (2014). Analysis of stage and clinical/prognostic factors for lung cancer from SEER registries: AJCC staging and collaborative stage data collection system. Cancer, 120 Suppl 23, 3781-3792. doi:10.1002/cncr.29045

Chen, X., Liao, R., Li, D., \& Sun, J. (2017). Induced cancer stem cells generated by radiochemotherapy and their therapeutic implications. Oncotarget, 8(10), 17301-17312. doi:10.18632/oncotarget.14230

Chen, X. L., Wei, S. S., Ji, Y. L., Guo, X. J., \& Yang, F. Q. (2015). Quantitative proteomics using SILAC: Principles, applications, and developments. Proteomics, 15(18), 3175-3192. doi:10.1002/pmic.201500108

Cho, W. C. (2017). Mass spectrometry-based proteomics in cancer research. Expert Rev Proteomics, 14(9), 725-727. doi:10.1080/14789450.2017.1365604

Chou, T. C. (2006). Theoretical basis, experimental design, and computerized simulation of synergism and antagonism in drug combination studies. Pharmacol Rev, 58(3), 621-681. doi:10.1124/pr.58.3.10

Chou, T. C. (2010). Drug combination studies and their synergy quantification using the Chou-Talalay method. Cancer Res, 70(2), 440-446. doi:10.1158/0008-5472.CAN-09-1947

Cihoric, N., Savic, S., Schneider, S., Ackermann, I., Bichsel-Naef, M., Schmid, R. A., . . Tapia, C. (2014). Prognostic role of FGFR1 amplification in early-stage non-small cell lung cancer. Br J Cancer, 110(12), 2914-2922. doi:10.1038/bjc.2014.229

Colell, A., Ricci, J. E., Tait, S., Milasta, S., Maurer, U., Bouchier-Hayes, L., . . G Green, D. R. (2007). GAPDH and autophagy preserve survival after apoptotic cytochrome $c$ release in the absence of caspase activation. Cell, 129(5), 983-997. doi:10.1016/j.cell.2007.03.045

Corso, J., Pan, K. T., Walter, R., Doebele, C., Mohr, S., Bohnenberger, H., . . O Oellerich, T. (2016). Elucidation of tonic and activated B-cell receptor signaling in Burkitt's lymphoma provides insights into regulation of cell survival. Proc Natl Acad Sci U S A, 113(20), 5688-5693. doi:10.1073/pnas.1601053113

Cox, J., \& Mann, M. (2008). MaxQuant enables high peptide identification rates, individualized p.p.b.range mass accuracies and proteome-wide protein quantification. Nat Biotechnol, 26(12), 1367-1372. doi:10.1038/nbt.1511

Craig, D. W., Nasser, S., Corbett, R., Chan, S. K., Murray, L., Legendre, C., . . Liang, W. S. (2016). A somatic reference standard for cancer genome sequencing. Sci Rep, 6, 24607. doi:10.1038/srep24607 
de Graauw, M., van Miltenburg, M. H., Schmidt, M. K., Pont, C., Lalai, R., Kartopawiro, J., . . van de Water, B. (2010). Annexin A1 regulates TGF-beta signaling and promotes metastasis formation of basal-like breast cancer cells. Proc Natl Acad Sci U S A, 107(14), 6340-6345. doi:10.1073/pnas.0913360107

Dennis, G., Sherman, B. T., Hosack, D. A., Yang, J., Gao, W., Lane, H. C., \& Lempicki, R. A. (2003). DAVID: Database for Annotation, Visualization, and Integrated Discovery. Genome Biology, 4(9). doi:10.1186/gb-2003-4-9-r60

Drake, J. M., Paull, E. O., Graham, N. A., Lee, J. K., Smith, B. A., Titz, B., . . Stuart, J. M. (2016). Phosphoproteome Integration Reveals Patient-Specific Networks in Prostate Cancer. Cell, 166(4), 1041-1054. doi:10.1016/j.cell.2016.07.007

Du, Z., \& Lovly, C. M. (2018). Mechanisms of receptor tyrosine kinase activation in cancer. Mol Cancer, 17(1), 58. doi:10.1186/s12943-018-0782-4

Dutt, A., Ramos, A. H., Hammerman, P. S., Mermel, C., Cho, J., Sharifnia, T., . . Meyerson, M. (2011). Inhibitor-sensitive FGFR1 amplification in human non-small cell lung cancer. PLoS One, 6(6), e20351. doi:10.1371/journal.pone.0020351

Eissmann, M., Melzer, I. M., Fernandez, S. B., Michel, G., Hrabe de Angelis, M., Hoefler, G., . . Zornig, M. (2013). Overexpression of the anti-apoptotic protein AVEN contributes to increased malignancy in hematopoietic neoplasms. Oncogene, 32(20), 2586-2591. doi:10.1038/onc.2012.263

Elakad, O., Lois, A. M., Schmitz, K., Yao, S., Hugo, S., Lukat, L., . . Bohnenberger, H. (2020). Fibroblast growth factor receptor 1 gene amplification and protein expression in human lung cancer. Cancer Med. doi:10.1002/cam4.2994

Ficarro, S. B., McCleland, M. L., Stukenberg, P. T., Burke, D. J., Ross, M. M., Shabanowitz, J., . . White, F. M. (2002). Phosphoproteome analysis by mass spectrometry and its application to Saccharomyces cerevisiae. Nat Biotechnol, 20(3), 301-305. doi:10.1038/nbt0302-301

Fishilevich, S., Zimmerman, S., Kohn, A., Iny Stein, T., Olender, T., Kolker, E., . . Lancet, D. (2016). Genic insights from integrated human proteomics in GeneCards. Database (Oxford), 2016. doi:10.1093/database/baw030

Fumarola, C., Cretella, D., La Monica, S., Bonelli, M. A., Alfieri, R., Caffarra, C., . . Ardizzoni, A. (2017). Enhancement of the anti-tumor activity of FGFR1 inhibition in squamous cell lung cancer by targeting downstream signaling involved in glucose metabolism. Oncotarget, 8(54), 9184191859. doi:10.18632/oncotarget.19279

Gandara, D. R., Hammerman, P. S., Sos, M. L., Lara, P. N., Jr., \& Hirsch, F. R. (2015). Squamous cell lung cancer: from tumor genomics to cancer therapeutics. Clin Cancer Res, 21(10), 22362243. doi:10.1158/1078-0432.CCR-14-3039

Geiger, T., Cox, J., Ostasiewicz, P., Wisniewski, J. R., \& Mann, M. (2010). Super-SILAC mix for quantitative proteomics of human tumor tissue. Nat Methods, 7(5), 383-385. doi:10.1038/nmeth.1446

Georgescu, M. M. (2010). PTEN Tumor Suppressor Network in PI3K-Akt Pathway Control. Genes Cancer, 1(12), 1170-1177. doi:10.1177/1947601911407325

Gronberg, B. H., Sundstrom, S., Kaasa, S., Bremnes, R. M., Flotten, O., Amundsen, T., . . Jordhoy, M. (2010). Influence of comorbidity on survival, toxicity and health-related quality of life in patients with advanced non-small-cell lung cancer receiving platinum-doublet chemotherapy. Eur J Cancer, 46(12), 2225-2234. doi:10.1016/j.ejca.2010.04.009

Guffanti, F., Chila, R., Bello, E., Zucchetti, M., Zangarini, M., Ceriani, L., . . Damia, G. (2017). In Vitro and In Vivo Activity of Lucitanib in FGFR1/2 Amplified or Mutated Cancer Models. Neoplasia, 19(1), 35-42. doi:10.1016/j.neo.2016.11.008

Haupl, B., Urlaub, H., \& Oellerich, T. (2019). Phosphoproteomic Analysis of Signaling Pathways in Lymphomas. Methods Mol Biol, 1956, 371-381. doi:10.1007/978-1-4939-9151-8_19

He, Y., Zhou, Z., Hofstetter, W. L., Zhou, Y., Hu, W., Guo, C., . . Fang, B. (2012). Aberrant expression of proteins involved in signal transduction and DNA repair pathways in lung cancer and their 
association with clinical parameters. PLoS One, 7(2), e31087. doi:10.1371/journal.pone.0031087

Heist, R. S., Mino-Kenudson, M., Sequist, L. V., Tammireddy, S., Morrissey, L., Christiani, D. C., . . . lafrate, A. J. (2012). FGFR1 amplification in squamous cell carcinoma of the lung. J Thorac Oncol, 7(12), 1775-1780. doi:10.1097/JTO.0b013e31826aed28

Ho, C. S., Lam, C. W., Chan, M. H., Cheung, R. C., Law, L. K., Lit, L. C., . . Tai, H. L. (2003). Electrospray ionisation mass spectrometry: principles and clinical applications. Clin Biochem Rev, 24(1), 312.

Holohan, C., Van Schaeybroeck, S., Longley, D. B., \& Johnston, P. G. (2013). Cancer drug resistance: an evolving paradigm. Nat Rev Cancer, 13(10), 714-726. doi:10.1038/nrc3599

Huang, W.-C., \& Hung, M.-C. (2009). Induction of Akt Activity by Chemotherapy Confers Acquired Resistance. Journal of the Formosan Medical Association, 108(3), 180-194. doi:10.1016/s0929-6646(09)60051-6

IARC, I. A. f. r. o. C., WHO. (2018). Latest global cancer data: Cancer burden rises to 18.1 million new cases and 9.6 million cancer deaths in 2018 [Press release]

Inamura, K. (2017). Lung Cancer: Understanding Its Molecular Pathology and the 2015 WHO Classification. Front Oncol, 7, 193. doi:10.3389/fonc.2017.00193

Jacobsen, K., Bertran-Alamillo, J., Molina, M. A., Teixido, C., Karachaliou, N., Pedersen, M. H., . . . Rosell, R. (2017). Convergent Akt activation drives acquired EGFR inhibitor resistance in lung cancer. Nat Commun, 8(1), 410. doi:10.1038/s41467-017-00450-6

Kamps, R., Brandao, R. D., Bosch, B. J., Paulussen, A. D., Xanthoulea, S., Blok, M. J., \& Romano, A. (2017). Next-Generation Sequencing in Oncology: Genetic Diagnosis, Risk Prediction and Cancer Classification. Int J Mol Sci, 18(2). doi:10.3390/ijms18020308

Katoh, M. (2019). Fibroblast growth factor receptors as treatment targets in clinical oncology. Nat Rev Clin Oncol, 16(2), 105-122. doi:10.1038/s41571-018-0115-y

Kellie, J. F., Tran, J. C., Lee, J. E., Ahlf, D. R., Thomas, H. M., Ntai, I., . . Kelleher, N. L. (2010). The emerging process of Top Down mass spectrometry for protein analysis: biomarkers, proteintherapeutics, and achieving high throughput. Mol Biosyst, 6(9), 1532-1539. doi:10.1039/c000896f

Khoury, G. A., Baliban, R. C., \& Floudas, C. A. (2011). Proteome-wide post-translational modification statistics: frequency analysis and curation of the swiss-prot database. Sci Rep, 1. doi:10.1038/srep00090

Kim, S., Jee, K., Kim, D., Koh, H., \& Chung, J. (2001). Cyclic AMP Inhibits Akt Activity by Blocking the Membrane Localization of PDK1. Journal of Biological Chemistry, 276(16), 12864-12870. doi:10.1074/jbc.M001492200

Kim, S. M., Kim, H., Yun, M. R., Kang, H. N., Pyo, K. H., Park, H. J., . . Cho, B. C. (2016). Activation of the Met kinase confers acquired drug resistance in FGFR-targeted lung cancer therapy. Oncogenesis, 5(7), e241. doi:10.1038/oncsis.2016.48

Kohler, L. H., Mireskandari, M., Knosel, T., Altendorf-Hofmann, A., Kunze, A., Schmidt, A., . . . Petersen, I. (2012). FGFR1 expression and gene copy numbers in human lung cancer. Virchows Arch, 461(1), 49-57. doi:10.1007/s00428-012-1250-y

Kohn, A. D., Summers, S. A., Birnbaum, M. J., \& Roth, R. A. (1996). Expression of a constitutively active Akt Ser/Thr kinase in 3T3-L1 adipocytes stimulates glucose uptake and glucose transporter 4 translocation. J Biol Chem, 271(49), 31372-31378. doi:10.1074/jbc.271.49.31372

Kosti, I., Jain, N., Aran, D., Butte, A. J., \& Sirota, M. (2016). Cross-tissue Analysis of Gene and Protein Expression in Normal and Cancer Tissues. Sci Rep, 6, 24799. doi:10.1038/srep24799

Kotani, H., Ebi, H., Kitai, H., Nanjo, S., Kita, K., Huynh, T. G., . . Y Yano, S. (2016). Co-active receptor tyrosine kinases mitigate the effect of FGFR inhibitors in FGFR1-amplified lung cancers with low FGFR1 protein expression. Oncogene, 35(27), 3587-3597. doi:10.1038/onc.2015.426 
Kozielski, J., Kaczmarczyk, G., Porebska, I., Szmygin-Milanowska, K., \& Golecki, M. (2012). Lung cancer in patients under the age of 40 years. Contemp Oncol (Pozn), 16(5), 413-415. doi:10.5114/wo.2012.31770

Kummar, S., \& Doroshow, J. H. (2013). Molecular targets in cancer therapy. Expert Rev Anticancer Ther, 13(3), 267-269. doi:10.1586/era.12.170

Kurzrock, R., Kantarjian, H. M., Kesselheim, A. S., \& Sigal, E. V. (2020). New drug approvals in oncology. Nat Rev Clin Oncol, 17(3), 140-146. doi:10.1038/s41571-019-0313-2

Kwek, S. S., Roy, R., Zhou, H., Climent, J., Martinez-Climent, J. A., Fridlyand, J., \& Albertson, D. G. (2009). Co-amplified genes at $8 p 12$ and $11 q 13$ in breast tumors cooperate with two major pathways in oncogenesis. Oncogene, 28(17), 1892-1903. doi:10.1038/onc.2009.34

Lemmon, M. A., \& Schlessinger, J. (2010). Cell signaling by receptor tyrosine kinases. Cell, 141(7), 1117-1134. doi:10.1016/j.cell.2010.06.011

Ligon, W. V. (2001). Liquid Chromatography Mass Spectrometry. In Encyclopedia of Materials: Science and Technology (pp. 1-5).

Lin, J. J., Zhu, V. W., Schoenfeld, A. J., Yeap, B. Y., Saxena, A., Ferris, L. A., . . Riely, G. J. (2018). Brigatinib in Patients With Alectinib-Refractory ALK-Positive NSCLC. J Thorac Oncol, 13(10), 1530-1538. doi:10.1016/j.jtho.2018.06.005

Liu, H., Ai, J., Shen, A., Chen, Y., Wang, X., Peng, X., . . Geng, M. (2017). c-Myc Alteration Determines the Therapeutic Response to FGFR Inhibitors. Clin Cancer Res, 23(4), 974-984. doi:10.1158/1078-0432.CCR-15-2448

Liu, X., Taftaf, R., Kawaguchi, M., Chang, Y. F., Chen, W., Entenberg, D., . . Liu, H. (2019). Homophilic CD44 Interactions Mediate Tumor Cell Aggregation and Polyclonal Metastasis in PatientDerived Breast Cancer Models. Cancer Discov, 9(1), 96-113. doi:10.1158/2159-8290.CD-180065

Lizio, M., Harshbarger, J., Shimoji, H., Severin, J., Kasukawa, T., Sahin, S., . . consortium, F. (2015). Gateways to the FANTOM5 promoter level mammalian expression atlas. Genome Biol, 16, 22. doi:10.1186/s13059-014-0560-6

Lopez, E., Wang, X., Madero, L., Lopez-Pascual, J., \& Latterich, M. (2012). Functional phosphoproteomic mass spectrometry-based approaches. Clin Transl Med, 1(1), 20. doi:10.1186/2001-1326-1-20

Lv, D., Guo, L., Zhang, T., \& Huang, L. (2017). PRAS40 signaling in tumor. Oncotarget, 8(40), 6907669085. doi:10.18632/oncotarget.17299

Malchers, F., Ercanoglu, M., Schutte, D., Castiglione, R., Tischler, V., Michels, S., . . Thomas, R. K. (2017). Mechanisms of Primary Drug Resistance in FGFR1-Amplified Lung Cancer. Clin Cancer Res, 23(18), 5527-5536. doi:10.1158/1078-0432.CCR-17-0478

Mao, Y., Yang, D., He, J., \& Krasna, M. J. (2016). Epidemiology of Lung Cancer. Surg Oncol Clin N Am, 25(3), 439-445. doi:10.1016/j.soc.2016.02.001

Minguez, P., Parca, L., Diella, F., Mende, D. R., Kumar, R., Helmer-Citterich, M., . . Bork, P. (2012). Deciphering a global network of functionally associated post-translational modifications. Mol Syst Biol, 8, 599. doi:10.1038/msb.2012.31

Mittra, I., Pal, K., Pancholi, N., Shaikh, A., Rane, B., Tidke, P., . . Nair, N. K. (2017). Prevention of chemotherapy toxicity by agents that neutralize or degrade cell-free chromatin. Ann Oncol, 28(9), 2119-2127. doi:10.1093/annonc/mdx318

Mohr, S., Doebele, C., Comoglio, F., Berg, T., Beck, J., Bohnenberger, H., . . Oellerich, T. (2017). Hoxa9 and Meis1 Cooperatively Induce Addiction to Syk Signaling by Suppressing miR-146a in Acute Myeloid Leukemia. Cancer Cell, 31(4), 549-562 e511. doi:10.1016/j.ccell.2017.03.001

Monaco, S. E., Rodriguez, E. F., Mahaffey, A. L., \& Dacic, S. (2016). FGFR1 Amplification in Squamous Cell Carcinoma of the Lung with Correlation of Primary and Metastatic Tumor Status. Am J Clin Pathol, 145(1), 55-61. doi:10.1093/ajcp/aqv013

Nesverova, V., \& Tornroth-Horsefield, S. (2019). Phosphorylation-Dependent Regulation of Mammalian Aquaporins. Cells, 8(2). doi:10.3390/cells8020082 
NIH. (2018). SEER Cancer Statistics Review (CSR) 1975-2015. Retrieved from https://seer.cancer.gov/archive/csr/1975 2015/\#contents

NIH. (2019). Targeted Cancer Therapies. Retrieved from https://www.cancer.gov/aboutcancer/treatment/types/targeted-therapies/targeted-therapies-fact-sheet\#what-aretargeted-cancer-therapies

Nilsson, T., Mann, M., Aebersold, R., Yates, J. R., 3rd, Bairoch, A., \& Bergeron, J. J. (2010). Mass spectrometry in high-throughput proteomics: ready for the big time. Nat Methods, 7(9), 681685. doi:10.1038/nmeth0910-681

Nogova, L., Sequist, L. V., Perez Garcia, J. M., Andre, F., Delord, J. P., Hidalgo, M., . . Wolf, J. (2017). Evaluation of BGJ398, a Fibroblast Growth Factor Receptor 1-3 Kinase Inhibitor, in Patients With Advanced Solid Tumors Harboring Genetic Alterations in Fibroblast Growth Factor Receptors: Results of a Global Phase I, Dose-Escalation and Dose-Expansion Study. J Clin Oncol, 35(2), 157-165. doi:10.1200/JCO.2016.67.2048

Olshina, M. A., \& Sharon, M. (2016). Mass Spectrometry: A Technique of Many Faces. Q Rev Biophys, 49. doi:10.1017/S0033583516000160

Ornitz, D. M., \& Itoh, N. (2015). The Fibroblast Growth Factor signaling pathway. Wiley Interdiscip Rev Dev Biol, 4(3), 215-266. doi:10.1002/wdev.176

Paik, P. K., Shen, R., Berger, M. F., Ferry, D., Soria, J. C., Mathewson, A., . . Andre, F. (2017). A Phase Ib Open-Label Multicenter Study of AZD4547 in Patients with Advanced Squamous Cell Lung Cancers. Clin Cancer Res, 23(18), 5366-5373. doi:10.1158/1078-0432.CCR-17-0645

Palmer, A. C., \& Sorger, P. K. (2017). Combination Cancer Therapy Can Confer Benefit via Patient-toPatient Variability without Drug Additivity or Synergy. Cell, 171(7), 1678-1691 e1613. doi:10.1016/j.cell.2017.11.009

Panis, C., Corrêa, S., Binato, R., \& Abdelhay, E. (2019). The Role of Proteomics in Cancer Research. In Oncogenomics (pp. 31-55).

Perez-Moreno, P., Brambilla, E., Thomas, R., \& Soria, J. C. (2012). Squamous cell carcinoma of the lung: molecular subtypes and therapeutic opportunities. Clin Cancer Res, 18(9), 2443-2451. doi:10.1158/1078-0432.CCR-11-2370

Pros, E., Lantuejoul, S., Sanchez-Verde, L., Castillo, S. D., Bonastre, E., Suarez-Gauthier, A., . . . Sanchez-Cespedes, M. (2013). Determining the profiles and parameters for gene amplification testing of growth factor receptors in lung cancer. Int J Cancer, 133(4), 898-907. doi:10.1002/ijc.28090

Qi, Z., Zhang, T., Song, L., Fu, H., Luo, H., Wu, J., . . . Huang, L. (2020). PRAS40 hyperexpression $\begin{array}{llll}\text { promotes hepatocarcinogenesis. } & \text { EBioMedicine, }\end{array}$ doi:10.1016/j.ebiom.2019.102604

Raina, D., Kosugi, M., Ahmad, R., Panchamoorthy, G., Rajabi, H., Alam, M., . . Kufe, D. (2011). Dependence on the MUC1-C oncoprotein in non-small cell lung cancer cells. Mol Cancer Ther, 10(5), 806-816. doi:10.1158/1535-7163.MCT-10-1050

Regad, T. (2015). Targeting RTK Signaling Pathways in Cancer. Cancers (Basel), 7(3), 1758-1784. doi:10.3390/cancers7030860

Rooney, C., Geh, C., Williams, V., Heuckmann, J. M., Menon, R., Schneider, P., . . Kilgour, E. (2016). Characterization of FGFR1 Locus in sqNSCLC Reveals a Broad and Heterogeneous Amplicon. PLoS One, 11(2), e0149628. doi:10.1371/journal.pone.0149628

Rush, J., Moritz, A., Lee, K. A., Guo, A., Goss, V. L., Spek, E. J., . . Comb, M. J. (2005). Immunoaffinity profiling of tyrosine phosphorylation in cancer cells. Nat Biotechnol, 23(1), 94-101. doi:10.1038/nbt1046

Russell, P. A., Yu, Y., Young, R. J., Conron, M., Wainer, Z., Alam, N., . . . Wright, G. M. (2014). Prevalence, morphology, and natural history of FGFR1-amplified lung cancer, including squamous cell carcinoma, detected by FISH and SISH. Mod Pathol, 27(12), 1621-1631. doi:10.1038/modpathol.2014.71

Sabari, J. K., \& Paik, P. K. (2017). Relevance of genetic alterations in squamous and small cell lung cancer. Ann Transl Med, 5(18), 373. doi:10.21037/atm.2017.06.72 
Saraswathy, N., \& Ramalingam, P. (2011). Phosphoproteomics. In Concepts and Techniques in Genomics and Proteomics (pp. 203-211).

Sasaki, H., Shitara, M., Yokota, K., Hikosaka, Y., Moriyama, S., Yano, M., \& Fujii, Y. (2012). Increased FGFR1 copy number in lung squamous cell carcinomas. Mol Med Rep, 5(3), 725-728. doi:10.3892/mmr.2011.715

Schildhaus, H. U., Heukamp, L. C., Merkelbach-Bruse, S., Riesner, K., Schmitz, K., Binot, E., . . . Buettner, R. (2012). Definition of a fluorescence in-situ hybridization score identifies highand low-level FGFR1 amplification types in squamous cell lung cancer. Mod Pathol, 25(11), 1473-1480. doi:10.1038/modpathol.2012.102

Schmid, P., Abraham, J., Chan, S., Wheatley, D., Brunt, M., \& Nemsadze, G. (2018). AZD5363 plus paclitaxel versus placebo plus paclitaxel as first-line therapy for metastatic triple-negative breast cancer (PAKT): A randomised, double-blind, placebo-controlled, phase II trial. Journal of Clinical Oncology, 36(15). doi:DOI 10.1200/JCO.2018.36.15_suppl.1007

Schultheis, A. M., Bos, M., Schmitz, K., Wilsberg, L., Binot, E., Wolf, J., . . Schildhaus, H. U. (2014). Fibroblast growth factor receptor 1 (FGFR1) amplification is a potential therapeutic target in small-cell lung cancer. Modern Pathology, 27(2), 214-221. doi:10.1038/modpathol.2013.141

Seo, A. N., Jin, Y., Lee, H. J., Sun, P. L., Kim, H., Jheon, S., . . Chung, J. H. (2014). FGFR1 amplification is associated with poor prognosis and smoking in non-small-cell lung cancer. Virchows Arch, 465(5), 547-558. doi:10.1007/s00428-014-1634-2

Sholl, L. M., Aisner, D. L., Varella-Garcia, M., Berry, L. D., Dias-Santagata, D., Wistuba, II, . . . Investigators, L. (2015). Multi-institutional Oncogenic Driver Mutation Analysis in Lung Adenocarcinoma: The Lung Cancer Mutation Consortium Experience. J Thorac Oncol, 10(5), 768-777. doi:10.1097/JTO.0000000000000516

Shruthi, B. S., Vinodhkumar, P., \& Selvamani. (2016). Proteomics: A new perspective for cancer. Adv Biomed Res, 5, 67. doi:10.4103/2277-9175.180636

Sica, G. L., \& Gal, A. A. (2012). Lung cancer staging: pathology issues. Semin Diagn Pathol, 29(3), 116126. doi:10.1053/j.semdp.2012.06.001

Singleton, K. R., Hinz, T. K., Kleczko, E. K., Marek, L. A., Kwak, J., Harp, T., . . Heasley, L. E. (2015). Kinome RNAi Screens Reveal Synergistic Targeting of MTOR and FGFR1 Pathways for Treatment of Lung Cancer and HNSCC. Cancer Res, 75(20), 4398-4406. doi:10.1158/00085472.CAN-15-0509

Socinski, M. A., Bondarenko, I., Karaseva, N. A., Makhson, A. M., Vynnychenko, I., Okamoto, I., . . . Renschler, M. F. (2012). Weekly nab-paclitaxel in combination with carboplatin versus solvent-based paclitaxel plus carboplatin as first-line therapy in patients with advanced nonsmall-cell lung cancer: final results of a phase III trial. J Clin Oncol, 30(17), 2055-2062. doi:10.1200/JCO.2011.39.5848

Somlyai, G., Collins, T. Q., Meuillet, E. J., Hitendra, P., D'Agostino, D. P., \& Boros, L. G. (2017). Structural homologies between phenformin, lipitor and gleevec aim the same metabolic oncotarget in leukemia and melanoma. Oncotarget, 8(30), 50187-50192. doi:10.18632/oncotarget.16238

Stokes, M. P., Farnsworth, C. L., Moritz, A., Silva, J. C., Jia, X., Lee, K. A., . . Comb, M. J. (2012). PTMScan direct: identification and quantification of peptides from critical signaling proteins by immunoaffinity enrichment coupled with LC-MS/MS. Mol Cell Proteomics, 11(5), 187-201. doi:10.1074/mcp.M111.015883

Szklarczyk, D., Gable, A. L., Lyon, D., Junge, A., Wyder, S., Huerta-Cepas, J., . . Mering, C. V. (2019). STRING v11: protein-protein association networks with increased coverage, supporting functional discovery in genome-wide experimental datasets. Nucleic Acids Res, 47(D1), D607D613. doi:10.1093/nar/gky1131

Tao, D., Han, X., Zhang, N., Lin, D., Wu, D., Zhu, X., . . Shi, Y. (2016). Genetic alteration profiling of patients with resected squamous cell lung carcinomas. Oncotarget, 7(24), 36590-36601. doi:10.18632/oncotarget.9096 
Torre, L. A., Siegel, R. L., \& Jemal, A. (2016). Lung Cancer Statistics. Adv Exp Med Biol, 893, 1-19. doi:10.1007/978-3-319-24223-1_1

Travis, W. D., Brambilla, E., \& Riely, G. J. (2013). New pathologic classification of lung cancer: relevance for clinical practice and clinical trials. J Clin Oncol, 31(8), 992-1001. doi:10.1200/JCO.2012.46.9270

Tucker, J. A., Klein, T., Breed, J., Breeze, A. L., Overman, R., Phillips, C., \& Norman, R. A. (2014). Structural insights into FGFR kinase isoform selectivity: diverse binding modes of AZD4547 and ponatinib in complex with FGFR1 and FGFR4. Structure, 22(12), 1764-1774. doi:10.1016/j.str.2014.09.019

Tyanova, S., \& Cox, J. (2018). Perseus: A Bioinformatics Platform for Integrative Analysis of Proteomics Data in Cancer Research. Methods Mol Biol, 1711, 133-148. doi:10.1007/978-14939-7493-1_7

Tyanova, S., Temu, T., Sinitcyn, P., Carlson, A., Hein, M. Y., Geiger, T., . . Cox, J. (2016). The Perseus computational platform for comprehensive analysis of (prote)omics data. Nat Methods, 13(9), 731-740. doi:10.1038/nmeth.3901

Tyers, M., \& Mann, M. (2003). From genomics to proteomics. Nature, 422(6928), 193-197. doi:10.1038/nature01510

Uhlen, M., Fagerberg, L., Hallstrom, B. M., Lindskog, C., Oksvold, P., Mardinoglu, A., . . Ponten, F. (2015). Proteomics. Tissue-based map of the human proteome. Science, 347(6220), 1260419. doi:10.1126/science.1260419

Van den Abbeele, A. D., Badawi, R. D., Tetrault, R. J., Cliche, J. P., Manola, J., Spangler, T., . . Demetri, G. D. (2003). FDG-PET as a surrogate marker for response to Gleevec (TM) (imatinib mesylate) in patients with advanced gastrointestinal stromal tumors (GIST). Journal of Nuclear Medicine, 44(5), 24p-25p.

Varsegi, G. M., \& Shidham, V. (2009). Cell block preparation from cytology specimen with predominance of individually scattered cells. J Vis $\operatorname{Exp}(29)$. doi:10.3791/1316

Vasaikar, S., Huang, C., Wang, X., Petyuk, V. A., Savage, S. R., Wen, B., . . Clinical Proteomic Tumor Analysis, C. (2019). Proteogenomic Analysis of Human Colon Cancer Reveals New Therapeutic Opportunities. Cell, 177(4), 1035-1049 e1019. doi:10.1016/j.cell.2019.03.030

von Massenhausen, A., Franzen, A., Heasley, L., \& Perner, S. (2013). FGFR1 as a novel prognostic and predictive biomarker in squamous cell cancers of the lung and the head and neck area. Ann Transl Med, 1(3), 23. doi:10.3978/j.issn.2305-5839.2013.06.08

Wang, H., Shi, T., Qian, W. J., Liu, T., Kagan, J., Srivastava, S., . . Camp, D. G., 2nd. (2016). The clinical impact of recent advances in LC-MS for cancer biomarker discovery and verification. Expert Rev Proteomics, 13(1), 99-114. doi:10.1586/14789450.2016.1122529

Wang, X., He, Y., Ye, Y., Zhao, X., Deng, S., He, G., . . L Liang, S. (2018). SILAC-based quantitative MS approach for real-time recording protein-mediated cell-cell interactions. Sci Rep, 8(1), 8441. doi:10.1038/s41598-018-26262-2

Wang, X., Zhang, H., \& Chen, X. (2019). Drug resistance and combating drug resistance in cancer. Cancer Drug Resistance. doi:10.20517/cdr.2019.10

Wang, Y., Gao, W., Xu, J. L., Chen, X. J., Yang, Y., Zhu, Y. Z., . . Liu, L. X. (2015). The Role of FGFR1 Gene Amplification as a Poor Prognostic Factor in Squamous Cell Lung Cancer: A MetaAnalysis of Published Data. Biomed Research International. doi:Artn 76308010.1155/2015/763080

Weiss, J., Sos, M. L., Seidel, D., Peifer, M., Zander, T., Heuckmann, J. M., . . Thomas, R. K. (2010). Frequent and focal FGFR1 amplification associates with therapeutically tractable FGFR1 dependency in squamous cell lung cancer. Sci Transl Med, 2(62), 62ra93. doi:10.1126/scitranslmed.3001451

Wilson, F. R., Coombes, M. E., Wylie, Q., Yurchenko, M., Brezden-Masley, C., Hutton, B., . . Cameron, C. (2017). Herceptin(R) (trastuzumab) in HER2-positive early breast cancer: protocol for a systematic review and cumulative network meta-analysis. Syst Rev, 6(1), 196. doi:10.1186/s13643-017-0588-2 
Wisniewski, J. R., \& Mann, M. (2016). A Proteomics Approach to the Protein Normalization Problem: Selection of Unvarying Proteins for MS-Based Proteomics and Western Blotting. J Proteome Res, 15(7), 2321-2326. doi:10.1021/acs.jproteome.6b00403

Woodard, G. A., Jones, K. D., \& Jablons, D. M. (2016). Lung Cancer Staging and Prognosis. Cancer Treat Res, 170, 47-75. doi:10.1007/978-3-319-40389-2_3

Wu, D. W., Wu, T. C., Chen, C. Y., \& Lee, H. (2016). PAK1 Is a Novel Therapeutic Target in Tyrosine Kinase Inhibitor-Resistant Lung Adenocarcinoma Activated by the PI3K/AKT Signaling Regardless of EGFR Mutation. Clin Cancer Res, 22(21), 5370-5382. doi:10.1158/10780432.CCR-15-2724

Wynes, M. W., Hinz, T. K., Gao, D., Martini, M., Marek, L. A., Ware, K. E., . . Heasley, L. E. (2014). FGFR1 mRNA and protein expression, not gene copy number, predict FGFR TKI sensitivity across all lung cancer histologies. Clin Cancer Res, 20(12), 3299-3309. doi:10.1158/10780432.CCR-13-3060

Xie, Z., Choong, P. F., Poon, L. F., Zhou, J., Khng, J., Jasinghe, V. J., . . Chen, C. S. (2008). Inhibition of CD44 expression in hepatocellular carcinoma cells enhances apoptosis, chemosensitivity, and reduces tumorigenesis and invasion. Cancer Chemother Pharmacol, 62(6), 949-957. doi:10.1007/s00280-008-0684-z

Yang, F., Gao, Y., Geng, J., Qu, D., Han, Q., Qi, J., \& Chen, G. (2013). Elevated expression of SOX2 and FGFR1 in correlation with poor prognosis in patients with small cell lung cancer. Int J Clin Exp Pathol, 6(12), 2846-2854.

Ye, D. Z., \& Field, J. (2012). PAK signaling in cancer. Cell Logist, 2(2), 105-116. doi:10.4161/cl.21882

Zappa, C., \& Mousa, S. A. (2016). Non-small cell lung cancer: current treatment and future advances. Transl Lung Cancer Res, 5(3), 288-300. doi:10.21037/tlcr.2016.06.07

Zhang, L. P., Yu, H., Badzio, A., Boyle, T. A., Schildhaus, H. U., Lu, X., . . Hirsch, F. R. (2015). Fibroblast Growth Factor Receptor 1 and Related Ligands in Small-Cell Lung Cancer. Journal of Thoracic Oncology, 10(7), 1083-1090. doi:10.1097/Jto.0000000000000562

Zhang, Y. C., Zhou, Q., \& Wu, Y. L. (2016). Emerging challenges of advanced squamous cell lung cancer. ESMO Open, 1(6), e000129. doi:10.1136/esmoopen-2016-000129

Zhang, Z., Wu, S., Stenoien, D. L., \& Pasa-Tolic, L. (2014). High-throughput proteomics. Annu Rev Anal Chem (Palo Alto Calif), 7, 427-454. doi:10.1146/annurev-anchem-071213-020216

Zheng, M. (2016). Classification and Pathology of Lung Cancer. Surgical Oncology Clinics of North America, 25(3), 447-+. doi:10.1016/j.soc.2016.02.003

Zhou, G. L., Zhuo, Y., King, C. C., Fryer, B. H., Bokoch, G. M., \& Field, J. (2003). Akt phosphorylation of serine 21 on Pak1 modulates Nck binding and cell migration. Mol Cell Biol, 23(22), 80588069. doi:10.1128/mcb.23.22.8058-8069.2003 